\title{
Search for Radiative $B$-Hadron Decays with the Collider Detector at Fermilab
}

\author{
Masashi TANAKA
}

A dissertation submitted to the Doctoral Program in Physics, the University of Tsukuba in partial fulfillment of the requirements for the degree of Doctor of Philosophy (Science)

January, 2001 


\section{Abstract}

Radiative decays of $B$ hadrons, $\bar{B}_{d} \rightarrow \bar{K}^{* 0} \gamma(\rho \gamma), \bar{B}_{s} \rightarrow \phi \gamma\left(\bar{K}^{* 0} \gamma\right)$, and $\Lambda_{b} \rightarrow$ $\Lambda \gamma(n \gamma)$, occur via the quark transition $b \rightarrow s(d)$ that involves a loop ("penguin") diagram. In the Standard Model (SM) the loop amplitude is dominated by a virtual intermediate top quark coupling to a $W^{-}$boson. Thus radiative $B$ decays are sensitive to the magnitude of the Cabibbo-Kobayashi-Maskawa (CKM) matrix elements $\left|V_{t s}\right|$ and $\left|V_{t d}\right|$. In addition, the existence of non-SM heavy charged particles such as a supersymmetric charged Higgs can affect the amplitude of the penguin loop. Measurements of radiative $B$ hadron decays, therefore, constitute low energy probes for physics beyond the SM.

In 1993, the CLEO collaboration observed the first radiative decays of $\bar{B}_{u}$ and $\bar{B}_{d}$ mesons. The three $B$ factories at the $\Upsilon(4 S)$ resonance, CLEO, BaBar, and BELLE, performed several measurements of the $\bar{B}_{u}$ and $\bar{B}_{d}$ radiative decays such as the branching fractions and the $\mathrm{CP}$ asymmetries. However, the $B$ factories can not produce the heavier hadrons such as the $\bar{B}_{s}$ and $\Lambda_{b}$. Therefore the measurements of the $\bar{B}_{s}$ and $\Lambda_{b}$ decays are unique at the high energy collider experiments. No radiative decays of $\bar{B}_{s}$ nor $\Lambda_{b}$ have been observed yet. From the search for $\bar{B}_{s} \rightarrow \phi \gamma$ decays, the DELPHI collaboration obtained $B\left(\bar{B}_{s} \rightarrow \phi \gamma\right)<7.0 \times 10^{-4}$ at $90 \%$ CL.

We searched for the radiative decays of $\bar{B}_{d} \rightarrow \bar{K}^{* 0}\left(\rightarrow K^{-} \pi^{+}\right) \gamma, \bar{B}_{s} \rightarrow \phi(\rightarrow$ $\left.K^{+} K^{-}\right) \gamma$, and $\Lambda_{b} \rightarrow \Lambda\left(\rightarrow p \pi^{-}\right) \gamma$. The data used in this analysis are collected with the Collider Detector at Fermilab (CDF) during 1994-95, corresponding to an integrated luminosity of $74 \mathrm{pb}^{-1}$ of $p \bar{p}$ collisions at $\sqrt{s}=1.8 \mathrm{TeV}$. In this analysis, the photon is identified by an electron-positron pair produced through the external photon conversion before the tracking detector volume. One of the conversion electrons serves as a trigger for recording the events. The $B$ hadrons are reconstructed by combining the momenta of the two conversion electrons and the two hadron tracks, where the two hadron tracks are constrained to intersect at a common point. The mass resolution of the reconstructed $B$ hadron is around $45 \mathrm{MeV} / c^{2}$. We infer the branching fractions of $B$ hadrons yielding radiative decays by forming ratios with the known branching fraction $B\left(\bar{B}_{u} \rightarrow J / \psi K^{-}\right)$, where the $J / \psi$ is reconstructed as $J / \psi \rightarrow e e$ decays. The topology observed in this "reference" channel is similar to the one resulting from the radiative decay. This way, any common factors cancel in the ratio of branching fractions, for example, the $B$ quark production cross section, and systematic uncertainties are reduced.

We optimize the selection cuts by maximizing the figure of merit defined by $\epsilon_{\mathrm{sig}} / \sqrt{\epsilon_{\mathrm{bg}}}$, 
iv

where $\epsilon_{\mathrm{sig}}$ and $\epsilon_{\mathrm{bg}}$ are, respectively, the efficiencies for the signal and background events found in the $\pm 100 \mathrm{MeV} / \mathrm{c}^{2}$ window around the $B$-hadron mass. After all the selection cuts, there are 1,0 , and 2 events of the $\bar{B}_{d}, \bar{B}_{s}$, and $\Lambda_{b}$ candidates with expected backgrounds of $0.6 \pm 0.3,0.1 \pm 0.1$, and $3.3 \pm 0.6$ events. We obtained upper limits on the branching fractions for the $\bar{B}_{d}, \bar{B}_{s}$, and $\Lambda_{b}$ radiative decays to be $1.9 \times 10^{-4}, 2.5 \times 10^{-4}$, and $1.3 \times 10^{-3}$ at the $90 \%$ confidence level. 


\section{Acknowledgements}

I would like to thank my thesis advisor Koji Takikawa for his guidance through my graduate school. I would like to thank Yoshihiro Seiya for his help through my research at Fermilab. Many crucial progresses on this study were obtained from his suggestion and advice. I would like to thank Barry Wicklund, the real expert of the $B$ physics, for his suggestion and advice. I respect his passion for and deep insight into the $B$ physics. I thank members of the $B$ physics group at CDF, which include Manfred Paulini and Jonathan Lewis, for their support and help.

In these days, experimental particle physics is no longer product for one person, but supported by hundreds of people. I would like to thank all of the people who have designed and constructed the Tevatron and CDF detector, took the data, written the analysis code, and so on.

I would like to thank stuff members of the CDF Japan group, Kunitaka Kondo, Shinhong Kim, Fumihiko Ukegawa, Kazuhiko Hara, and Makoto Shimojima. They provided me the opportunity to work at the CDF experiments.

I would like to thank CDF students which include Takashi Suzuki and Yoshiyuki Miyazaki. I could not take over the two year's life in Chicago, the severe winter, plain foods, etc, without their friendship. I would like to thank Carol Picciolo, Kyoko Kunori, Kazuko Kumashiro, and Mutsumi Uenishi. We, physicists, can do nothing without their help through their secretary works.

Finally I would like to thank my family for their support.

This work was supported by the U.S. Department of Energy and the National Science Foundation; the Natural Sciences and Engineering Research Council of Canada; the Istituto Nazionale di Fisica Nucleare of Italy; the Ministry of Education, Science, Sports and Culture of Japan; the National Science Council of the Republic of China; and the A. P. Sloan Foundation. 


\section{Contents}

List of Figures

$\mathbf{x v}$

List of Tables

xvi

1 Introduction 1

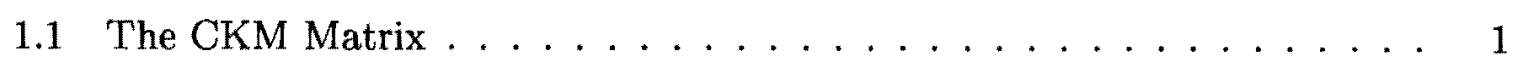

$1.2 \quad B$-Hadron Decays . . . . . . . . . . . . . . . . 6

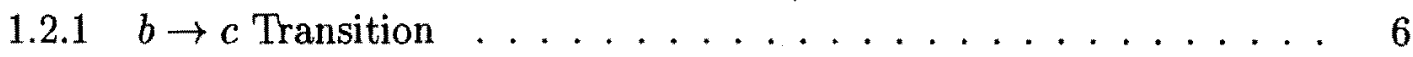

$1.2 .2 \quad b \rightarrow u$ Transition .................... 7

$1.2 .3 \quad b \rightarrow s(d)$ Transition . . . . . . . . . . . . . . 7

1.3 Radiative $B$ Decay within the Standard Model . . . . . . . . . . . 9 9

1.4 Radiative $B$ Decay Beyond the Standard Model . . . . . . . . . . . 11

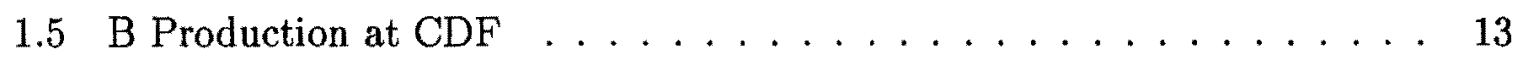

1.5.1 b-Quark Production . . . . . . . . . . . . . . . 13

$1.5 .2 \quad b$-Quark Fragmentation . . . . . . . . . . . . . 15

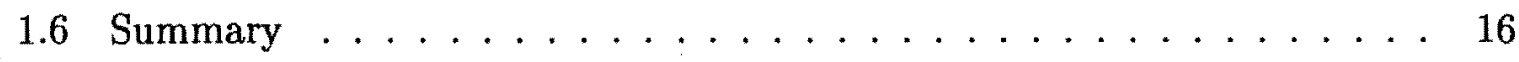

2 Experimental Apparatus $\quad 19$

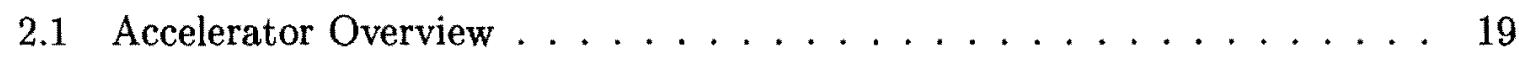

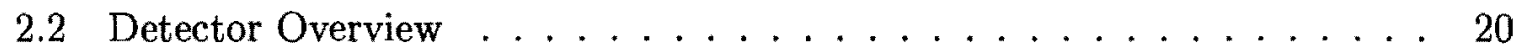

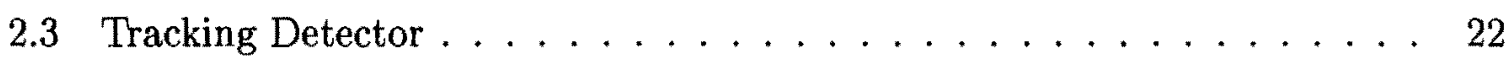

2.3.1 Silicon Vertex Detector $(\mathrm{SVX}) \ldots \ldots \ldots . \ldots . \ldots 24$

2.3.2 Vertex Time Projection Chamber (VTX) . . . . . . . . 25

2.3.3 Central Tracking Chamber (CTC) . . . . . . . . . . 26 
2.3 .4 Track Reconstruction . . . . . . . . . . . . . . 26

$2.3 .5 d E / d x \ldots \ldots \ldots \ldots \ldots \ldots \ldots \ldots \ldots \ldots \ldots$

2.3 .6 Photon Radiator . . . . . . . . . . . . . . . . 30

2.4 Calorimeters . . . . . . . . . . . . . . . . . 32

2.4.1 Central Electromagnetic Calorimeter (CEM) $\ldots \ldots \ldots \ldots \ldots$

2.4.2 Central Strip Chamber (CES) $\ldots \ldots \ldots \ldots \ldots \ldots \ldots$

2.4 .3 Central Hadronic Calorimeter (CHA) $\ldots \ldots \ldots \ldots . \ldots . \ldots 32$

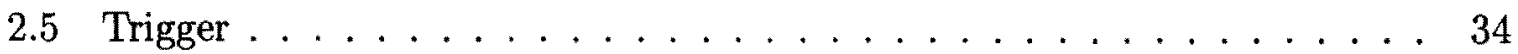

3 Event Reconstruction $\quad 35$

3.1 Event Selection Overview $\ldots \ldots \ldots \ldots \ldots$

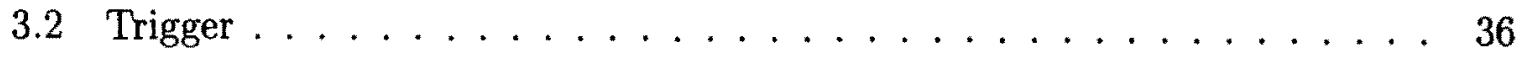

3.2 .1 Level $1 \ldots \ldots \ldots \ldots \ldots \ldots \ldots$

3.2 .2 Level $2 \ldots \ldots \ldots \ldots \ldots \ldots \ldots \ldots$

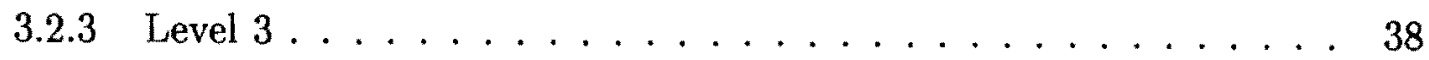

3.3 Monte Carlo Sample . . . . . . . . . . . . . . . . . . 40

$3.3 .1 \quad B$ Hadron Generator $\ldots \ldots \ldots \ldots$. . . . . . . . 40

3.3.2 Detector Simulation . . . . . . . . . . . . 40

3.4 Electron Reconstruction $\ldots \ldots \ldots$. . . . . . . . . . . . 41

3.4.1 Electromagnetic Energy: $E_{T} \ldots \ldots \ldots \ldots \ldots \ldots$

$3.4 .2 \quad E / p \ldots \ldots \ldots \ldots \ldots \ldots \ldots \ldots \ldots \ldots \ldots$

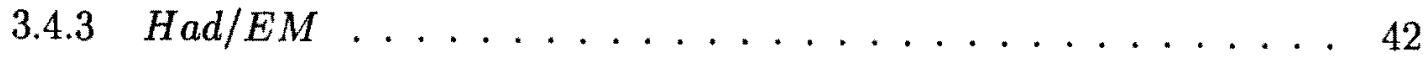

$3.4 .4 L_{\text {share }} \ldots \ldots \ldots \ldots \ldots \ldots \ldots \ldots \ldots \ldots \ldots$

$3.4 .5 \chi_{\text {strip,wire }}^{2} \ldots \ldots \ldots \ldots \ldots \ldots \ldots \ldots \ldots$

$3.4 .6 \quad \Delta z, \Delta x \ldots \ldots \ldots \ldots \ldots \ldots \ldots \ldots \ldots \ldots$

3.4.7 Comparison between the Data and MC . . . . . . . . . 43

3.5 Vertex Fitting . . . . . . . . . . . . . . . . . 45

$3.6 \quad J / \psi \rightarrow e^{+} e^{-} \ldots \ldots \ldots \ldots \ldots \ldots \ldots \ldots \ldots \ldots \ldots$

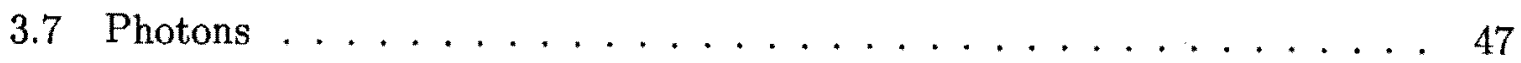


3.8 Radiative $B$ Signal Reconstruction $\ldots \ldots \ldots \ldots \ldots$. . . . . . . 49 3.8.1 $\bar{B}_{d} \rightarrow \bar{K}^{* 0} \gamma$ and $\bar{B}_{s} \rightarrow \phi \gamma \ldots \ldots \ldots . \ldots 49$

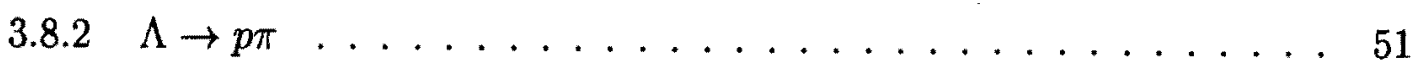

$3.8 .3 \Lambda_{b} \rightarrow \Lambda \gamma \ldots \ldots \ldots \ldots \ldots \ldots \ldots \ldots \ldots$

$3.9 \bar{B} \rightarrow J / \psi$ Decay Reconstruction . . . . . . . . . . . . 55

$3.9 .1 \bar{B}_{u} \rightarrow J / \psi K^{-} \ldots \ldots \ldots \ldots \ldots \ldots \ldots \ldots$

$3.9 .2 \bar{B}_{d} \rightarrow J / \psi \bar{K}^{* 0} \ldots \ldots \ldots \ldots \ldots \ldots \ldots \ldots$

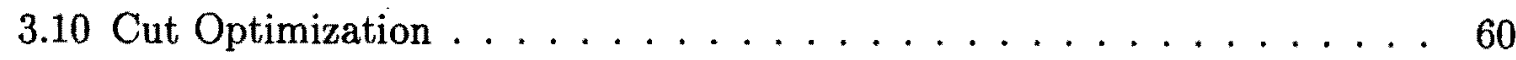

4 Estimating the Efficiencies $\quad \mathbf{7 0}$

4.1 Efficiencies Classification . . . . . . . . . . . . . . . 70

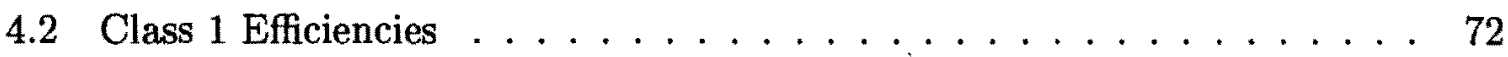

4.2 .1 Conversion Probability . . . . . . . . . . . . . 72

4.2.2 Soft Electron Quality Cut Efficiency for $J / \psi \rightarrow e e \ldots \ldots \ldots$. . 75

4.2 .3 Quality Cut Efficiency for $\Lambda \rightarrow p \pi \ldots \ldots \ldots \ldots 75$

4.3 Class 2 Efficiencies $\ldots \ldots \ldots \ldots \ldots \ldots \ldots \ldots$

4.3.1 Level 2 Trigger Efficiency $\ldots \ldots \ldots \ldots$. . . . . 81

4.3.2 Trigger Electron Quality Cut Efficiency . . . . . . . . . . . . 82

$4.3 .2 .1 \quad L_{\text {share }} \ldots \ldots \ldots \ldots \ldots \ldots \ldots \ldots$

$4.3 .2 .2 \quad H a d / E M \ldots \ldots \ldots \ldots \ldots \ldots \ldots$

4.4 Class 3 Efficiencies $\ldots \ldots \ldots \ldots \ldots \ldots$

4.5 Tracking Efficiencies $\ldots \ldots \ldots \ldots \ldots \ldots$

4.5.1 CTC Tracking Efficiencies $\ldots \ldots \ldots \ldots \ldots$

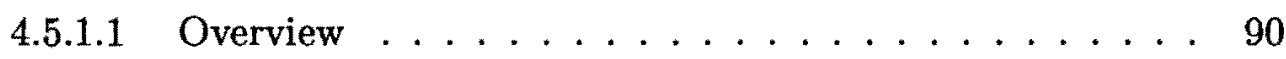

4.5.1.2 Conversion Tracking Efficiency $\ldots \ldots \ldots \ldots 92$

4.5.1.3 $\Lambda$ Tracking Efficiency . . . . . . . . . . . . . 93

4.5.2 SVX Tracking Efficiencies $\ldots \ldots \ldots \ldots \ldots \ldots$

4.5.2.1 Check of the SVX Efficiency . . . . . . . . . . . 102

$4.5 .3 \quad$ Vertex Fitting Efficiency . . . . . . . . . . . . . . . . . 104 
4.5.3.1 Overview . . . . . . . . . . . . . . . . . 104

4.5.3.2 Conversion Constraint Fitting Efficiency . . . . . . . 104

4.5.3.3 $\Lambda$ Vertex Constraint Fitting Efficiency . . . . . . . . 111

4.6 Summary of the Efficiency Estimation $\ldots \ldots \ldots 11$

5 Systematic Uncertainties $\quad 115$

5.1 Class 1 Systematics . . . . . . . . . . . . . . . . . . . 115

5.2 Class 2 Systematics . . . . . . . . . . . . . . . . . 115

$5.2 .1 \quad B$-Badron $p_{T}$ Distribution $\ldots \ldots \ldots \ldots \ldots \ldots$

5.3 Class 3 Systematics . . . . . . . . . . . . . . . . . . . 117

5.4 Summary of the Systematics . . . . . . . . . . . . . . . 117

6 Limit 118

$\begin{array}{lll}7 \text { Conclusions } & 121\end{array}$

A MC Material Calibration $\quad 123$

A.1 Biases to Radius Distribution . . . . . . . . . . . . . . 123

A.2 Counting the Conversion Rates . . . . . . . . . . . . . . 124

A.3 Results . . . . . . . . . . . . . . . . . . . . . 127

A.4 Vertex $\chi^{2}$ Cut Dependence . . . . . . . . . . . . . . . 129 


\section{List of Figures}

1.1 The unitarity triangle. $\ldots \ldots \ldots \ldots \ldots \ldots \ldots$

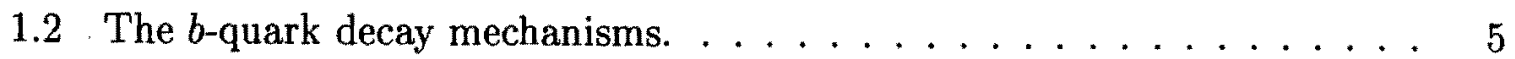

1.3 Penguin vertices resolved in terms of point-like vertices. . . . . . . 10

$1.4 B(b \rightarrow s \gamma)$ in the supergravity model as a function of the charged Higgs mass for $\tan \beta=2$ (top) and $\tan \beta=30$ (bottom). Each solid line shows the branching fraction in the two Higgs doublet model. Each dashed line shows the branching fraction in the SM. Dotted lines denote the upper and lower bounds on the branching fraction given by CLEO . . . . . . 12

1.5 Leading order Feynman diagrams depicting $b$-quark production by quarkantiquark annihilation (A), and gluon-gluon fusion (B and C) . . . . 14

1.6 Next-to-leading order Feynman diagrams depicting $b$-quark production by gluon radiation (A), gluon splitting (B), and flavor excitation (C) . . . 14

1.7 The $b$-quark production cross section at the CDF . . . . . . . 15

1.8 A schematic view of the fragmentation of $b$-quark into $B$ meson. . . . . 16

1.9 The Peterson fragmentation functions for three different $\epsilon$ 's. $\ldots \ldots 17$

1.10 The $B$-meson production cross section at the CDF. . . . . . . 17

2.1 A schematic view of Fermilab accelerator complex. . . . . . . . . . 21

2.2 The integrated luminosity delivered to the $\mathrm{B} 0$ collision point and the

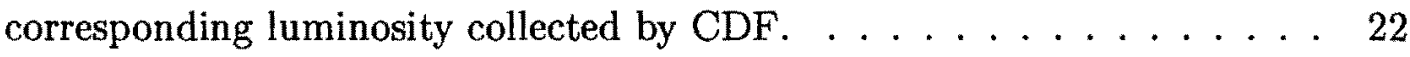

2.3 An isometric view of the CDF detector. . . . . . . . . . 23

2.4 A side-view cross section of the CDF detector. . . . . . . . . . . . . 23

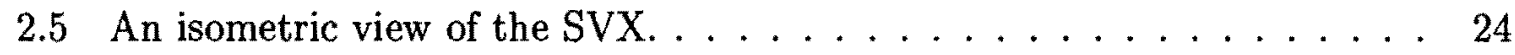

2.6 An isometric view of the SVX ladder. . . . . . . . . . 25

2.7 A schematic view of the VTX. . . . . . . . . . . . 25 


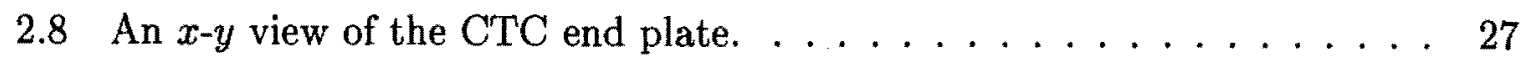

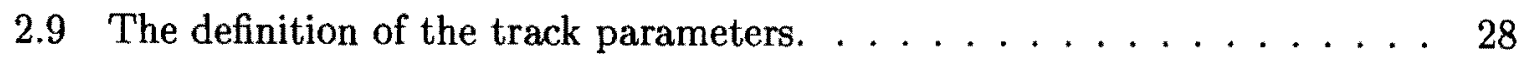

$2.10 \mathrm{dE} / \mathrm{dx}$ in the $8.5 \mathrm{~atm} \mathrm{Ar}-\mathrm{CH}_{4}$ as a function of the track momentum for several particles. . . . . . . . . . . . . . . . 29

$2.11 \mathrm{~A} x y$-view cross section of the CDF inner detector by using the photon conversion vertex. .................. 30

2.12 A $r z$-view cross section of the CDF inner detector by using the photon

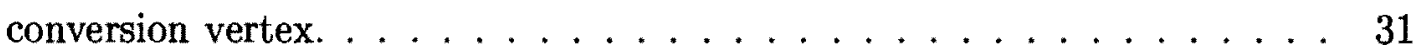

$2.13 \mathrm{~A}$ isometric view of the central calorimeter. . . . . . . . . . . 33

3.1 Schematic diagram of the $\bar{B}_{d} \rightarrow \bar{K}^{* 0} \gamma$ reconstruction. . . . . . . 37

3.2 Schematic diagram of the $\Lambda_{b} \rightarrow \Lambda \gamma$ reconstruction. $\ldots \ldots \ldots 37$

3.3 CFT bin $0-5$ efficiency curves as a function of the $p_{T} \ldots \ldots \ldots 39$

3.4 Distributions of the electron quality cut parameters, $E / p, \mathrm{Had} / E M$, and $L_{s h a r e}$, for the partner electron of the $J / \psi \rightarrow e e$ candidates (points) and the $J / \psi K$ MC sample (histograms). . . . . . . . . . . . . . . . 44

3.5 Distributions of the electron quality cut parameters, $\chi_{s t r i p, w i r e}^{2}, \Delta z, \Delta x$, for the partner electron of the $J / \psi \rightarrow e e$ candidates (points) and the $J / \psi K$ MC sample (histograms) . . . . . . . . . . . . . . 45

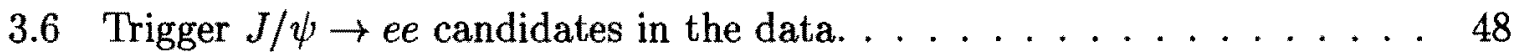

3.7 Schematic view of the effect of bremsstrahlung to the conversion reconstruction. . . . . . . . . . . . . . . . . . 49

3.8 Trigger $\gamma$ conversion candidates in Run Ib data. . . . . . . . . . . 50

$3.9 \bar{B}_{d} \rightarrow \bar{K}^{* 0} \gamma$ and $\bar{B}_{s} \rightarrow \phi \gamma$ mass distribution in Run IB data. . . . . . . 52

$3.10 \Lambda \rightarrow p \pi$ reconstruction in the SVX. . . . . . . . . . 54

$3.11 M_{\Lambda_{b}}$ distribution for the signal $\mathrm{MC}$ before and after the constraint fit that

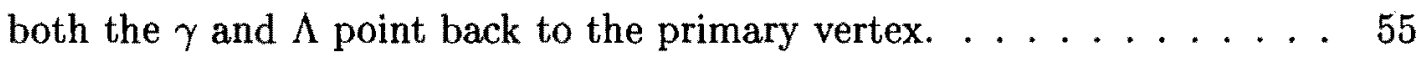

$3.12 M_{\Lambda}$ (left) and $M_{\Lambda_{b}}$ (right) distribution for $\Lambda_{b} \rightarrow \Lambda \gamma$ candidates with $\Lambda$ reconstructed using the CTC tracks (top) and SVX tracks (bottom). . 56

3.13 Correlation between of $M_{e e K}$ and $M_{e e}$ for the $J / \psi K$ candidates (top) and signal MC (bottom). The line shows $M_{e e K}-M_{e e}=M_{B_{u}}-M_{J / \psi} . \ldots .58$

$3.14 \bar{B}_{d} \rightarrow J / \psi \bar{K}^{* 0}$ and $\bar{B}_{u} \rightarrow J / \psi K^{-}$mass distribution in Run Ib data. . . 58 
3.15 Comparison of the data (points) and MC (histograms) for the $\bar{B}_{u} \rightarrow$ $J / \psi K^{-}$candidates in several reconstructed parameters. . . . . . . 59

$3.16 \bar{K}^{* 0} \gamma$ and $J / \psi K^{-}$mass distribution after the optimized cut. . . . . 62

3.17 The CTC display of a $\bar{K}^{* 0} \gamma$ candidate. . . . . . . . . . 63

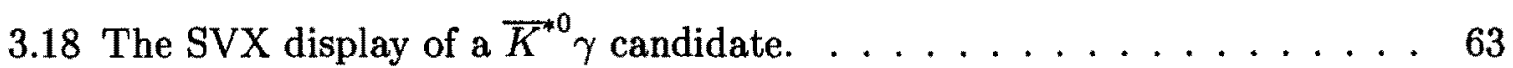

$3.19 \phi \gamma$ and $J / \psi K^{-}$mass distribution after the optimized cut. . . . . . . . 64

3.20 Definition of the signed impact parameter. . . . . . . . . . . 66

$3.21 M_{\Lambda}$ and $d_{0 \Lambda}$ distribution for the SVX $\Lambda_{b}$ candidates and the signal MC. . 66

$3.22 p_{T \Lambda}$ distributions for the CTC $\Lambda_{b}$ side band events and the signal MC. . . 67

$3.23 \epsilon_{s i g}, \epsilon_{b g}$, and fom distribution as a function of the $p_{T \Lambda}\left(d_{0 \Lambda}\right)$ for the cut optimization of the CTC (SVX) $\Lambda_{b} \ldots \ldots \ldots \ldots \ldots \ldots$

$3.24 M_{\Lambda}$ (left) and $M_{\Lambda_{b}}$ (right) distributions for $\Lambda_{b} \rightarrow \Lambda \gamma$ candidates after the optimized selection with $\Lambda$ reconstructed using the CTC tracks (top) and SVX tracks (bottom). . . . . . . . . . . . . . . . . 68

$3.25 \Lambda_{b} \rightarrow \Lambda \gamma$ and $\bar{B}_{u} \rightarrow J / \psi K^{-}$candidates after the optimized selection. . . 69

4.1 A $x y$-view of the photon conversion vertex distribution in the MC sample. 74

4.2 A $r z$-view of the photon conversion vertex distribution in the MC sample. 74

4.3 $d E / d x$ distribution for the partner track of the trigger conversion candidates (top), and the efficiency of $d E / d x>-1.27$ as a function of track

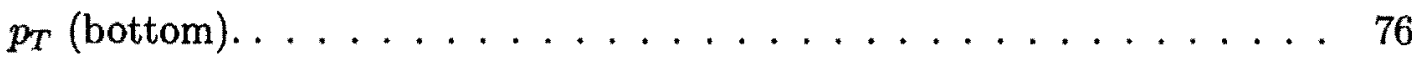

4.4 $M_{e e}$ distribution for the trigger $J / \psi \rightarrow e e$ candidates before and after $E / p>0.5$ cut for the partner track (top), and the cut efficiency as a function of track $p_{T}$ (bottom) . . . . . . . . . . . 77

4.5 The $d E / d x$ distributions for the $\Lambda \rightarrow p \pi$ signal (histograms), and the ackground estimated from the $\Lambda$ mass side band events (points). . . . 78

4.6 $\Lambda \rightarrow p \pi$ mass distribution before (points) and after (histogram) the $d E / d x$ and conversion removal cuts (top), and the efficiency as a function

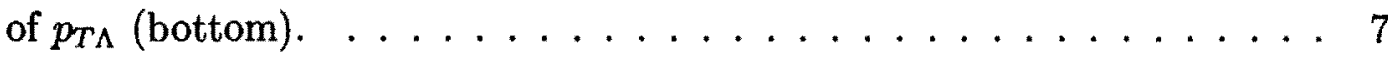

4.7 Impact parameter significance distributions for the proton and pion from the $\Lambda$ 
$4.8 d_{0} / \sigma$ cut efficiency for $\Lambda \rightarrow p \pi$ as a function of $p_{T \Lambda}$ for the data (top) and the MC (bottom) . . . . . . . . . . . . . . 80

4.9 L2 CEM_8_CFT_7_5_XCES trigger efficiency as a function of $E_{T}$ and $p_{T}$. 82

4.10 Distributions of the electron quality cut parameters for the partner electron of the $J / \psi \rightarrow e e$ candidates (points) and the $J / \psi K$ MC sample

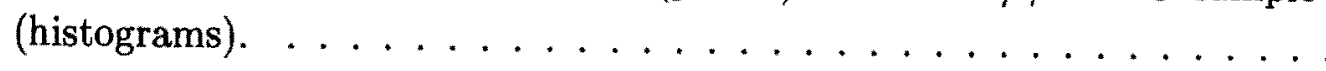

$4.11 L_{\text {share }}$ cut efficiency of the trigger electron of the $K^{*} \gamma$ and $J / \psi K \mathrm{MC}$. The plot when the electrons are in a same wedge is also shown (points). .

$4.12 L_{\text {share }}$ cut efficiency as a function of the cut value for the $J / \psi \rightarrow e e$ candidates and $J / \psi K \mathrm{MC}$ sample. A plot when the electrons are in a same wedge is also shown (bottom) . . . . . . . . . 85

$4.13 \mathrm{Had} / \mathrm{EM}$ distribution for partner electrons of the $J / \psi \rightarrow e e$ candidates and trigger electrons of the $K^{*} \gamma, J / \psi K$, and $J / \psi K^{*}$ MC. . . . . 87

$4.14 N_{t r k}$ distribution for partner electrons of the $J / \psi \rightarrow e e$ candidates and

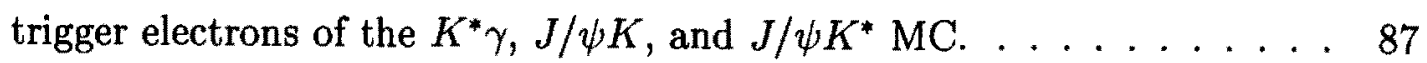

$4.15 \mathrm{Had} / \mathrm{EM}$ cut efficiency for partner electrons of the $J / \psi \rightarrow e e$ candidates 88

4.16 The $\bar{B}_{u}$ mass distributions before (points) and after (histogram) the $B$ isolation cut (top), and the $I_{B}$ distribution (bottom) obtained from the $\bar{B}_{u} \rightarrow J / \psi K^{-}$candidates in data. The arrow in the bottom plot corre-

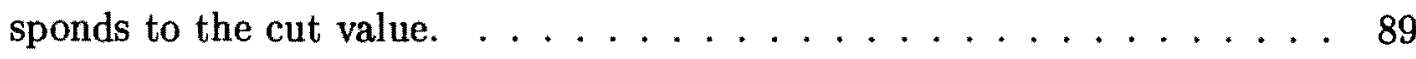

4.17 The same plots as the Figure 4.16 with differenc $p_{T}(B)$ regions, $p_{T}(B)<$

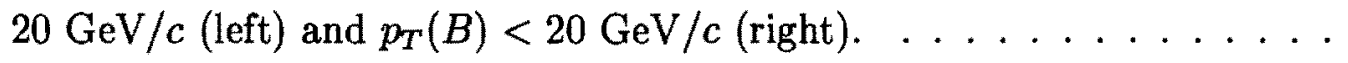

$4.18 p_{T}(B)$ distributions for the radiative $B$ decays (points) and the reference signals (histograms) after all the selection cut obtained from the $\mathrm{MC}$ sample. 90

4.19 CTC track reconstruction efficiency as a function of the track $p_{T}$ for positively (solid line) and negatively (dashed line) charged tracks ( [41], Figure $5)$.

4.20 Integrated luminosity, instantaneous luminosity, and conversion rate run by run for those runs in which there are at least 500 conversion candidates. 94

4.21 Scatter plots for the conversion rate in the various $r$ region as a function of the instantaneous luminosity. . . . . . . . . . . . . . 95

4.22 Fraction of the conversion rate in the CTC can as a function of the instantaneous luminosity (top) and the run number (bottom) . . . . . 96 
4.23 Dielectron mass distributions for the $J / \psi \rightarrow e e$ candidates for the six different run periods. . . . . . . . . . . . . . 97

4.24 The $J / \psi \rightarrow e e$ and conversion rate a run by run. . . . . . . 97

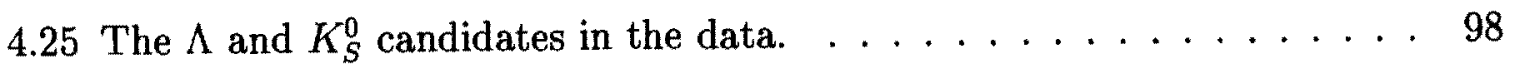

4.26 Impact parameter and $z$ distribution of the $\Lambda$ candidates around the trigger electron primary vertex. . . . . . . . . . . . 99

4.27 Vertex radius distributions for the $\Lambda$ and $K_{S}^{0}$ candidates in the strange mass signal and side band events (histograms) in the data. The points show the radius distribution after background subtraction using the side band events. . . . . . . . . . . . . . . 100

4.28 Run by run rates of the $\Lambda \rightarrow p \pi$ and $K_{S}^{0}$ events and teir ratio. . . . 100

4.29 Instantaneous luminosity dependence of the $\Lambda \rightarrow p \pi$ and $K_{S}^{0}$ rates and their ratio. . . . . . . . . . . . . . . . . . . 101

4.30 Run by run rate of the $\Lambda \rightarrow p \pi$ events and that with decay radius $>20$ $\mathrm{cm}$, and their ratio. . . . . . . . . . . . . . . 101

-4.31 Instantaneous luminosity dependence of the $\Lambda \rightarrow p \pi$ events and that with decay radius $>20 \mathrm{~cm}$, and their ratio. . . . . . . . . 102

4.32 The SVX efficiency for the $J / \psi \rightarrow e e$ candidates and the $J / \psi K$ signal MC sample. . . . . . . . . . . . . . . . . . . . 103

4.33 Radius distributions of the conversion vertex for the different fitting CL regions. . . . . . . . . . . . . . . . 105

4.34 Conversion constraint fitting CL distribution in the CTC can radius signal region events (solid histogram) and side band events (dashed histogram), and the CL cut efficiency curve (bottom plot). . . . . . . 106

$4.35 z$ of the conversion vertex distributions in the VTX radius for the different fitting $\mathrm{CL}$ region. . . . . . . . . . . . 107

4.36 Conversion constraint fitting probability cut efficiency in the VTX radius. 107

$4.37|z|$ of the conversion vertex distributions in the SVX radius for the different fitting CL region. . . . . . . . . . . 108

4.38 Conversion constraint fitting probability cut efficiency in the VTX radius. 108

4.39 Conversion constraint fitting CL cut efficiency in the various radius regions. 109 
4.40 Conversion constraint fitting CL cut efficiency as a function of the vertex radius.

4.41 Conversion constraint fitting CL cut efficiency obtained from the data (solid line) and MC (dashed line). . . . . . . . . . . . . . . 110

4.42 Common vertex constraint fitting CL cut efficiency for the $J / \psi \rightarrow e e$ obtained from the data (solid line) and MC (dashed line). . . . . . . 111

4.43 The CL distribution for the $K_{S}^{0}$ (top) and $\Lambda$ (bottom) vertex constraint fit in the strange meson mass signal and side band events (histograms).

The real distributions after background subtraction are shown as points. 112

4.44 CL cut efficiencies for the $K_{S}^{0}$ and $\Lambda$ vertex constraint fit (lines), and those for the events with decay radius $>20 \mathrm{~cm}$ (points). . . . . . . . . 113

$6.1 N_{90 \%}$ and $N_{95 \%}$ as a function of the systematic uncertainty. . . . . 119

$6.2 N_{90 \%}$ and $N_{95 \%}$ with $N_{o b s}=2, N_{b g}=3.4 \pm 0.6$, and $\sigma_{s y s}=43 \%$. . 120

A.1 $p_{T}$ distribution for the trigger $\gamma$ conversion candidates in the Run IB data and MC. . . . . . . . . . . . . . . . . 124

A.2 Dalitz event subtraction. . . . . . . . . . . . . . 125

A.3 Distribution of the conversion vertex radius. . . . . . . . . . . 126

A.4 $\phi$ distribution of the conversion vertex. . . . . . . . . . . . 126

A.5 $\phi$ distribution of the conversion vertex around SVX cable. . . . . . . 127

A.6 Distribution of the conversion vertex radius around the CTC can for the SVX cable region (top-left histogram), for the outside the SVX cable region (top-right histogram). The "real" $\mathrm{R}$ distributions for the SVX cable (top points) and CTC can (bottom) are also shown. . . . . . . . . 128

A.7 Material thickness without the SVX fiducial requirement for the data (black circles) and MC (open circles). . . . . . . . . . . . . 130

A.8 Material thickness with the SVX fiducial requirement for the data (black circles) and $\mathrm{MC}$ (open circles). . . . . . . . . . . . . 131 


\section{List of Tables}

2.1 Cross section and signal rate for various physics processes. . . . . . 34

3.1 Summary of the mass and width of the bottom and strange hadrons. . . 36

3.2 Electron quality cut in Level 3 and offline selections. . . . . . . . . 44

3.3 Event selection criteria. . . . . . . . . . . . . . . . . . 47

3.4 Reconstruction criteria for the radiative $B$ decays. . . . . . . . . . 52

3.5 Reconstruction criteria for the $\Lambda_{b} \rightarrow \Lambda \gamma \ldots \ldots \ldots \ldots$

3.6 Cut optimization for $\bar{B}_{d} \rightarrow \bar{K}^{* 0} \gamma \ldots \ldots \ldots \ldots \ldots \ldots \ldots \ldots \ldots \ldots$

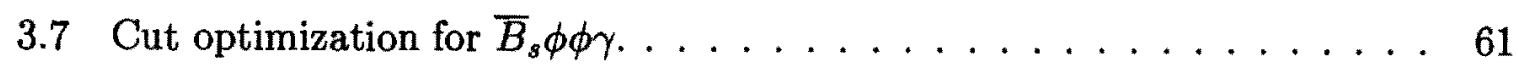

4.1 Ingredients for the calculation of the branching fractions of $\bar{B}_{d} \rightarrow \bar{K}^{* 0} \gamma$, $\bar{B}_{s} \rightarrow \phi \gamma$, and $\Lambda_{b} \rightarrow \Lambda \gamma$ decays according to Eqs. (4.5)-(4.7). . . . 114

5.1 Summary of the systematic uncertainties. . . . . . . . . 117

A.1 Results for material thickness estimation. . . . . . . . . . . . . . 129 


\section{Chapter 1}

\section{Introduction}

In this chapter, we describe the physics background of the radiative $B$ decays. The Standard Model and new physics interpretation of the radiative decays, and also the production mechanism of the $B$ hadrons in hadron colliders are discussed.

\subsection{The CKM Matrix}

In the Standard Model $(\mathrm{SM})[1]$ of $\mathrm{SU}(3)_{\mathrm{C}} \times \mathrm{SU}(2)_{\mathrm{L}} \times \mathrm{U}(1)_{\mathrm{Y}}$ gauge symmetry with three fermion generations, the interactions of quarks with the $\mathrm{SU}(2)_{\mathrm{L}}$ gauge bosons $\left(W_{\mu}^{a}\right)$ are given by

$$
\mathcal{L}_{W}=-\frac{1}{2} g \gamma^{\mu} \tau^{a} W_{\mu}^{a} \overline{\chi_{i L}} 1_{i j} \chi_{j L}+\text { h.c. }
$$

where $\gamma^{\mu}$ ( $\mu=1$ to 4$)$ operates in Lorentz space, $\tau^{a}(a=1,2,3)$ operates in $S U(2)_{L}$ space, $1_{i j}$ ( $i$ and $j=1$ to 3 ) is the unit matrix operating in generation space, and $\chi_{i L}$ are the fundamental left-handed quark states of the unbroken electroweak theory:

$$
\chi_{i L}=\left(\begin{array}{c}
u_{1} \\
d_{1}
\end{array}\right)_{L}\left(\begin{array}{c}
u_{2} \\
d_{2}
\end{array}\right)_{L}\left(\begin{array}{c}
u_{3} \\
d_{3}
\end{array}\right)_{L}
$$

The interactions of quarks with the single Higgs scalar doublet $\phi=\left(\phi^{+}, \phi^{0}\right)$ are given by

$$
\mathcal{L}_{Y}=-G_{i j} \overline{\chi_{i L}} \phi d_{j R}-F_{i j} \overline{\chi_{i L}} \bar{\phi} u_{j R}+h . c .
$$

where $G_{i j}$ and $F_{i j}$ are general complex $3 \times 3$ matrices and $u_{i R}\left(d_{i R}\right)$ represent the foundmental right-handed up type (down type) quark states of the unbroken electroweak theory:

$$
\begin{aligned}
& u_{i R}=u_{1 R}, u_{2 R}, u_{3 R} \\
& d_{i R}=d_{1 R}, d_{2 R}, d_{3 R} .
\end{aligned}
$$


With the spontaneous symmetry breaking:

$$
\phi=\left(\begin{array}{l}
\phi^{ \pm} \\
\phi^{0}
\end{array}\right) \rightarrow \sqrt{\frac{1}{2}}\left(\begin{array}{l}
0 \\
v
\end{array}\right)
$$

the mass terms are given by

$$
\mathcal{L}_{M}=-M_{d i j} \overline{d_{i L}} d_{j R}-M_{u i j} \overline{u_{i L}} u_{j R}+\text { h.c. }
$$

where the mass matrices, $M_{d}$ and $M_{u}$, are defined by

$$
M_{d}=G v / \sqrt{2}, \quad M_{u}=F v / \sqrt{2}
$$

Then the interaction of the quarks with the charged gauge boson $\left(W^{ \pm}\right)$is given by

$$
\mathcal{L}_{W}=-\sqrt{\frac{1}{2}} g \gamma^{\mu} W_{\mu}^{+} \overline{u_{i L}} 1_{i j} d_{j L}+\text { h.c. }
$$

The SM does not give any predictions on the $3 \times 3$ mass matrices $M_{d}$ and $M_{u}$, thus the SM does not predict the quark mass. The mass matrices are not necessary to be diagonal matrices, and can have complex elements. These are sources of the quark generation mixing and the CP violation in the SM.

In the minimal Standard Model, neutrino masses are assumed to be zero. It means the mass matrix for the neutrino was thought to be zero. However recent observation of the disappearance of the second generation neutrino [2] in atmospheric neutrinos at Super Kamiokande (SK) experiment shows that the charged current interaction of the leptons also has similar structure as the quark sector, and it requires a certain extension of the current Standard Model with mass-less neutrinos.

Any complex matrix can be transformed to a diagonal matrix by multiplying on the left and right by appropriate unitary matrices. Thus by unitary transformations on the foundmental quark states of the unbroken electroweak theory,

$$
\begin{aligned}
& \left(\begin{array}{l}
u_{1} \\
u_{2} \\
u_{3}
\end{array}\right)_{L, R}=V_{u L, R}\left(\begin{array}{l}
u \\
c \\
t
\end{array}\right)_{L, R} \\
& \left(\begin{array}{l}
d_{1} \\
d_{2} \\
d_{3}
\end{array}\right)_{L, R}=V_{d L, R}\left(\begin{array}{l}
d \\
s \\
b
\end{array}\right)_{L, R}
\end{aligned}
$$


we can transform $M_{u}$ and $M_{d}$ to diagonal forms

$$
V_{d L}^{\dagger} M_{d} V_{d R}=M_{d}^{\text {diag }}, \quad V_{u L}^{\dagger} M_{u} V_{u R}=M_{u}^{\text {diag }},
$$

where $M_{u, d}^{\text {diag }}$ are diagonal and real, and the diagonal elements correspond to the quark masses, while $V_{u L, R}$ and $V_{d L, R}$ are complex. The charged current interactions are given in the mass eigenbasis $\left(u_{L}, c_{L}, t_{L}, d_{L}, s_{L}\right.$, and $\left.b_{L}\right)$ by

$$
\mathcal{L}_{W}=-\frac{1}{2} g \gamma^{\mu} W_{\mu}^{+} \overline{(u, c, t)_{L}} V_{C K M}\left(\begin{array}{l}
d \\
s \\
b
\end{array}\right)_{L}+\text { h.c. }
$$

The matrix $V_{C K M}=V_{u L}^{\dagger} V_{d L}$, so called as the Cabibbo-Kobayashi-Maskawa (CKM) matrix, is the mixing matrix for three quark generations and given an explicit parameterization by Kobayashi and Maskawa in 1973 [3]. It depends on four parameters: three can be chosen as real angles (like the Cabibbo angle [4]) and one is phase.

By convention the CKM matrix is expressed by,

$$
V_{C K M}=\left(\begin{array}{lll}
V_{u d} & V_{u s} & V_{u b} \\
V_{c d} & V_{c s} & V_{c b} \\
V_{t d} & V_{t s} & V_{t b}
\end{array}\right)
$$

and Kobayashi and Maskawa originally chose a parameterization involving the four parameters, $\theta_{1}, \theta_{2}, \theta_{3}$, and $\delta$,

$$
\left(\begin{array}{ccc}
c_{1} c_{3} & s_{1} c_{3} & s_{3} e^{-i \delta} \\
-s_{1} c_{2}-c_{1} s_{2} s_{3} e^{i \delta} & c_{1} c_{2}-s_{1} s_{2} s_{3} e^{i \delta} & s_{2} c_{3} \\
s_{1} s_{2}+c_{1} c_{2} s_{3} e^{i \delta} & -c_{1} s_{2}-s_{1} c_{2} s_{3} e^{i \delta} & c_{2} c_{3}
\end{array}\right),
$$

where $c_{i}=\cos \theta_{i}$ and $s_{i}=\sin \theta_{i}$. Several experimental and phenomenological results have shown that $s_{3}$ and $s_{2}$ are small numbers: $\mathcal{O}\left(10^{-3}\right)$ and $\mathcal{O}\left(10^{-2}\right)$, respectively. Consequently, $c_{2}=c_{3}=1$ is a good approximation, and the four independent parameters are given as

$$
s_{1}=\left|V_{u s}\right|, \quad s_{2}=\left|V_{c b}\right|, \quad s_{3}=\left|V_{u b}\right|, \quad \delta .
$$

The Wolfenstein parameterization [5] is an approximate parametrization of the CKM matrix in which each element is expanded as a power series in the small parameter $\lambda=\left|V_{u s}\right|=0.22$,

$$
\left(\begin{array}{ccc}
1-\frac{\lambda^{2}}{2} & \lambda & A \lambda^{3}(\rho-i \eta) \\
-\lambda & 1-\frac{\lambda^{2}}{2} & A \lambda^{2} \\
A \lambda^{3}(1-\rho-i \eta) & -A \lambda^{2} & 1
\end{array}\right)+\mathcal{O}\left(\lambda^{4}\right)
$$


and the set (1.17) is replaced by,

$$
\lambda, \quad A, \quad \rho, \quad \eta,
$$

Because of the smallness of $\lambda$ and the fact that for each element the expansion parameter is actually $\lambda^{2}$, it is sufficient to keep only the first few terms in this expansion.

Since the existence of the fourth generation quarks, new gauge bosons, etc, causes violations of the unitarity of the CKM matrix, it is a good test of the Standard Model to show the unitarity of the CKM matrix. Several experimental and theoretical efforts to show the unitarity are ongoing. The unitarity of the CKM matrix leads various relations between its elements. In paticular, we have

$$
V_{u d} V_{u b}^{*}+V_{c d} V_{c b}^{*}+V_{t d} V_{t b}^{*}=0
$$

Since (1.20) contains both $V_{u b}$ and $V_{t d}$, which are expected to have the large phases, it is an interesting equation. We usually interpret the equation (1.20) as 'unitarity triangle' by assigning each of the terms as one side of the triangle. The Wolfenstein parameterization offers in conjunction with the unitarity triangle a very transparent geometrical representation of the structure of the CKM matrix. This turns out to be very useful in the phenomenology of rare decays and of CP violation. Equation (1.20) is transformed as follows,

$$
\begin{array}{r}
1+V_{u d} V_{u b}^{*} / V_{c d} V_{c b}^{*}++V_{t d} V_{t b}^{*} / V_{c d} V_{c b}^{*}=0 \\
1+(-\rho-i \eta)+(-1+\rho+i \eta)=0 .
\end{array}
$$

Figure 1.1 shows the triangle $(1.20)$ drawn in the $\rho-\eta$ plane. There have been several experimental efforts to show the unitarity of the triangle such as, the semileptonic $B$ decay rates, rare $B$ and $K$ meson decays, and mixing and CP violation in the $B_{d}-\bar{B}_{d}$, $B_{s}-\bar{B}_{s}$ and $K^{0}-\bar{K}^{0}$ system. Using the constraints from these measurements together with unitarity, and assuming only three generations, the $90 \%$ confidence level limits on the magnitude of the elements of the matrix are [6],

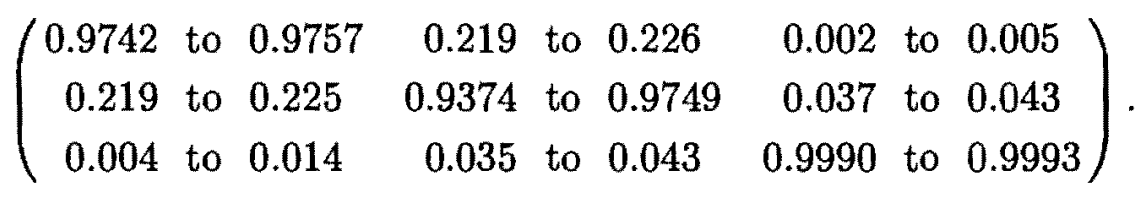




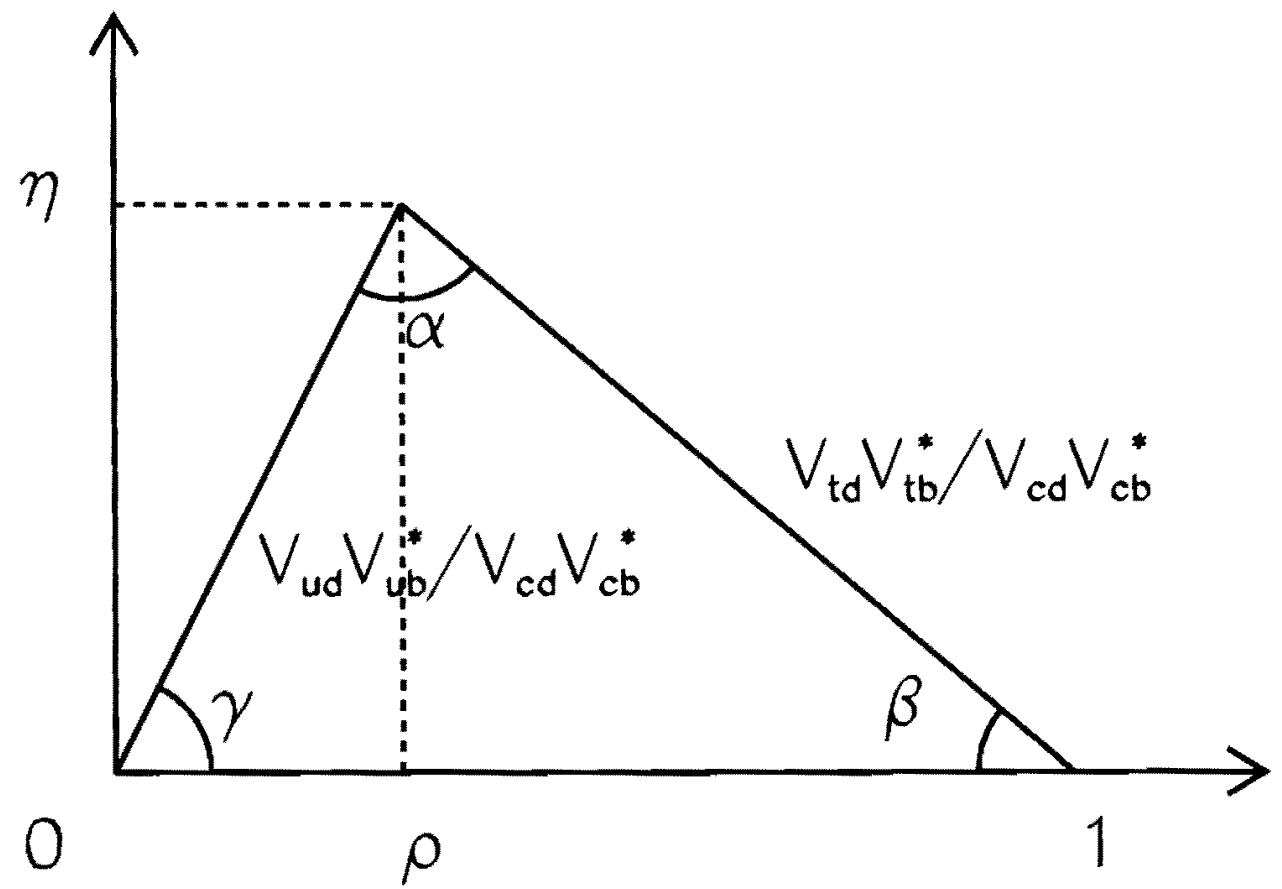

Figure 1.1: The unitarity triangle.

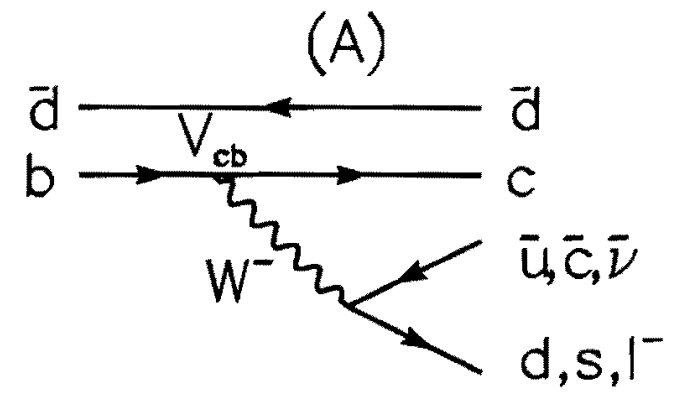

(C)

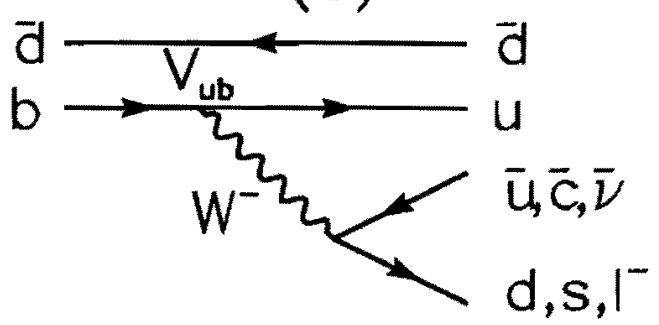

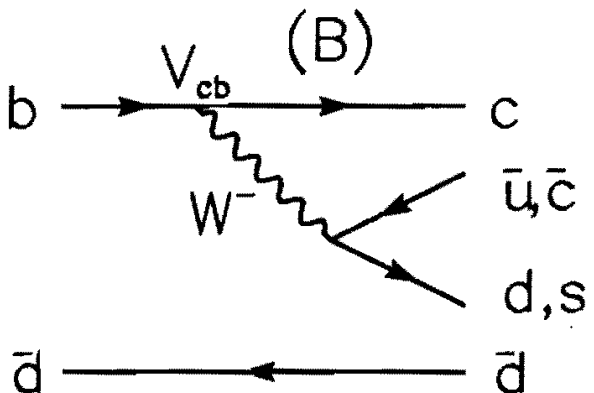

(D)

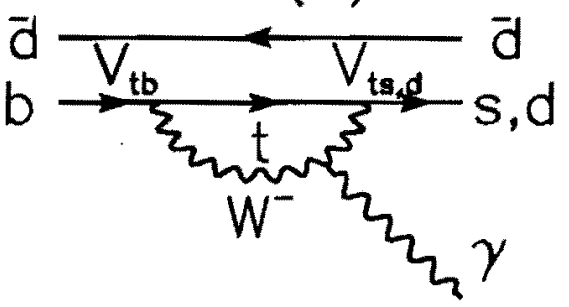

Figure 1.2: The $b$-quark decay mechanisms. 


\section{$1.2 \quad B$-Hadron Decays}

The bottom ( $b$ ) quark was first observed in 1977 at Fermilab through a narrow resonance at an energy of about $9.5 \mathrm{GeV} / c^{2}$ in $p+$ nucleus $\rightarrow \mu^{+} \mu^{-}+\mathrm{X}$ [7]. The resonance was interpreted a $b \bar{b}$ state, and called $\Upsilon$. Since then several mesons and baryons which includes the $b$ quark, $\bar{B}_{u}=\bar{u} b, \bar{B}_{d}=\bar{d} b, \bar{B}_{s}=\bar{s} b, \bar{B}_{c}=\bar{c} b, \Lambda_{b}=u d b$, and $\Xi_{b}=u s b$ are observed and their production and decay mechanisms were studied.

\subsection{1 $\quad b \rightarrow c$ Transition}

$B$-hadron decays occur primarily through the $b \rightarrow c$ transition. In such decays the dominant weak diagram is the spectator diagram, shown in Figure 1.2(A), where the virtual $W^{-}$materializes into either a $\bar{u} d$ or $\bar{c} s$ quark pair or a lepton and antineutrino pair. In hadronic decays, the quark pair becomes one of the final state hadrons, while the $c$ quark pairs with the spectator antiquark to form the other hadron. In semileptonic decays, the $c$ quark and the spectator antiquark hadronized independently of the leptonic current.

The extraction of the Standard Model parameters from the experimental results is complicated by the fact that only $B$ hadron can be studied and not free $b$ quarks. The light quarks and the gluons surrounding the $b$ quark in the $B$ hadron gives rise to significant corrections that have to be taken into account. Since leptons do not interact strongly, the semileptonic $B$ hadron decays are less affected by these QCD corrections and the theoretical calculations are more reliable.

The strength of the $b \rightarrow c$ coupling, i.e. $\left|V_{c b}\right|$, is determined by several measurements of semileptonic decay rates by the CLEO and LEP, and its present status [6] is

$$
\left|V_{c b}\right|=0.040 \pm 0.002
$$

where the uncertainty are rather dominated by the model dependence of the theoretical calculation to extract the matrix element from the decay rate measurement. Within the next 3-5 years, the theoretical error might be reduced to $2-3 \%$ [8].

In hadronic decays, there is "color suppressed" diagram shown in Figure 1.2(B), which has different set of quark pairings. In this diagram, the spectator anti-quark and the quark from the $W^{-}$become one of the final state hadrons. Observation of $B \rightarrow J / \psi X_{s}$ decays, where $X_{s}$ is a strange meson and its branching fraction is $\sim 1 \%$, gives experimental evidence for the presence of this diagram. 
One of such decays, the neutral $B_{d}$ meson decay to a CP eigenstate of $J / \psi K_{S}^{0}$, is interesting for the study of CP violation in $B_{d}$ decays, namely to extract the angle $\beta=\arg \left(V_{t d}\right)$ in the unitarity triangle. The limit of the $\beta$ was recently set by the $\mathrm{CDF}$ collaboration at the $95 \%$ confidence level (CL) to be $\sin 2 \beta>0$ [9]. Also several experiments such as the BaBar and Belle are planning to measure the $\sin 2 \beta$ with $<0.1$ of precision within next few years.

\subsection{2 $b \rightarrow u$ Transition}

All $B$-hadron decays that do not occur through the usual $b \rightarrow c$ decay are known as rare $B$ decays. The simplest diagram for such decay is obtained by the CKM suppressed $b \rightarrow u$ transition (Figure 1.2(C)). These decays probe the magnitude of $V_{u b}$. It sets bounds on the combination $\rho^{2}+\eta^{2}=\left|V_{u b} / \lambda V_{c b}\right|^{2}$ in the Wolfenstein parameterization (1.18), which corresponds to the length of one of the tree sides of the unitarity triangle (Figure 1.1).

The semileptonic $b \rightarrow u$ decay rates are measured by the CLEO and LEP, and its present status $[6]$ is

$$
\left|V_{u b} / V_{c b}\right|=0.090 \pm 0.025
$$

where the uncertainty is dominated by the model dependence of the theoretical calculation to extract the matrix element from the decay rate measurement. Within the next $3-5$ years, the theoretical error might be reduced to $\sim 10 \%[8]$.

The two body decay of the neutral $B$ meson to a CP eigenstate, $B_{d} \rightarrow \pi^{+} \pi^{-}$, which occur through the $b \rightarrow u$ transition, is interesting for the study of CP violation in $B$ decays, namely to extract the angle $\alpha$ in the unitarity triangle. It actually gives $\beta+\gamma=\arg \left(V_{t d} V_{u b}\right)$ which corresponds to $\alpha$ by assuming the unitarity of the CKM matrix. The decay was recently observed by the CLEO collaboration with a branching fraction of $\sim 5 \times 10^{-6}[10]$.

\subsection{3 $\quad b \rightarrow s(d)$ Transition}

In the Standard Model, only the $W^{ \pm}$boson is allowed to cause the flavor-changing currents, and the flavor-changing neutral current (FCNC) is suppressed and such transitions can only be induced by higher order processes. One way is the so-called penguin process which, in $b$ quark decays, results in effective FCNC $b \rightarrow s$ or $b \rightarrow d$ transitions with the emission and reabsorption of a $W$ boson. A photon, gluon, or $Z$ boson is emitted from 
the loop, and the presence of a photon, gluon, or $Z$ boson results in electromagnetic, gluonic, and weak penguin processes, respectively. The more massive the particles participating in the loop are, the higher the branching fraction for such processes. Thus the top quark dominates the diagrams in the SM and the penguin decays provide an access to the $V_{t s}$ and $V_{t d}$. Figure 1.2(D) shows the Feynman diagram for the radiative $b \rightarrow s \gamma$ decays induced by the electromagnetic penguin diagram.

The observation of the decay $B^{0, \pm} \rightarrow K^{*}(892) \gamma$, reported in 1993 by the CLEO experiment with the branching fraction of $\sim 5 \times 10^{-5}$ [11], was the first evidence for the penguin diagram. They recently updated the measurement of the branching fraction for the $B^{0, \pm} \rightarrow K^{*} \gamma$ decays to be $B\left(B^{0, \pm} \rightarrow K^{*} \gamma\right)=\left(4.55_{-0.68}^{+0.72} \pm 0.34\right) \times 10^{-5}$, where the first uncertainty is statistical and the second is systematic. The ratio of the CKM matrix element $\left|V_{t s}\right| /\left|V_{c s}\right|$ is extracted from the ratio of the decay rate measurements between the $B \rightarrow K^{*} \gamma$ and $D \rightarrow K^{* 0} \ell \nu[15]$ to be

$$
\frac{\left|V_{t s}\right|}{\left|V_{c s}\right|}=1.10 \pm 0.43
$$

where the large uncertainty $(39 \%)$ is dominated by the theoretical uncertainty $(38 \%)$.

The branching fraction for the inclusive radiative decays $B \rightarrow X_{s} \gamma$, where $X_{s}$ represents a collection of hadrons containing strange quarks, was also measured by CLEO to be $(3.15 \pm 0.35 \pm 0.32 \pm 0.26) \times 10^{-4}$ [13], where the first uncertainty is statistical, the second is systematic, and the third is for model dependence. No radiative decays of $B_{s}$ nor $\Lambda_{b}$ have been observed yet. From the search for $B_{s} \rightarrow \phi \gamma$ decays, the DELPHI collaboration obtained $B\left(B_{s} \rightarrow \phi \gamma\right)<7.0 \times 10^{-4}$ at the $90 \%$ CL [14].

The ratio $\left|V_{t d}\right| /\left|V_{t s}\right|$ is extracted from the ratio of the branching fractions between the $b \rightarrow d \gamma$ and $b \rightarrow s \gamma$. The CLEO collaboration reported $B(B \rightarrow \rho \gamma) / B\left(B \rightarrow K^{*} \gamma\right)<$ 0.32 at the $90 \%$ confidence level (CL) [12] and the result gives an upper limit,

$$
\frac{\left|V_{t d}\right|}{\left|V_{t s}\right|}=\sqrt{\xi \frac{B(B \rightarrow \rho \gamma)}{B\left(B \rightarrow K^{*} \gamma\right)}}<0.72 \text { at the } 90 \% \mathrm{CL}
$$

where $\xi=0.58$ is given by the theoretical calculation [16] of the form factors for $B \rightarrow K^{*}$ and $B \rightarrow \rho$. Since $K^{*}$ and $\rho$ have broad width, it is hard to separate these two decays experimentally. In the $B_{s}$ radiative decays, the $\left|V_{t d}\right| /\left|V_{t s}\right|$ is extracted from the ratio of the branching fractions between the $B_{s} \rightarrow K^{* 0} \gamma$ and $B_{s} \rightarrow \phi \gamma$. Since the $\phi\left(K^{*}\right)$ meson from the $B_{s}$ decay has narrow width compared to the $K^{*}(\rho)$ meson from the $B_{d}$ decay, it is easier to separate the $\phi$ and $K^{* 0}$ in the $B_{s}$ analysis. In this analysis the $B_{s}$ mass resolution is critical to separate the $B_{s} \rightarrow K^{* 0} \gamma$ decay from the $B_{d} \rightarrow K^{* 0} \gamma$ decay. 
Besides the $b \rightarrow u$ transition, the two-body hadronic decay, $B \rightarrow \pi \pi$, occurs by the $b \rightarrow d g$ penguin process. Thus, there is 'penguin pollution' for the measurement of the angle $\alpha$ in the $B \rightarrow \pi \pi$ decays. The phenomenological prediction for the size of the penguin pollution has large uncertainty. There are attempts to determine the pollution by using the penguin dominated channel such as $B \rightarrow K \pi$ and $B_{s} \rightarrow K K$.

The Standard Model favored interpretation of the $\nu_{\mu}$ disappearance in the SK experiment is $\nu_{\mu}-\nu_{\tau}$ mixing trough the $\nu_{\mu} \leftrightarrow \nu_{\tau}$ penguin transition. To check the appearance of the $\nu_{\tau}$ and the CP-violating effects in the $\nu_{\mu}-\nu_{\tau}$ system is an experimental target of the next decade. We also expect the FCNC $\mu \rightarrow e$ transition through the penguin loop which gives an interesting experimental signature of the $\mu \rightarrow e \gamma$.

\subsection{Radiative $B$ Decay within the Standard Model}

In this section, we describe the outline to calculate the amplitude of the radiative $B$ decays within the Standard Model [21]. There are several effort to understand the lowenergy QCD corrections to the penguin loop. Because of $\mathcal{O}\left(M_{b, c, s}\right) \ll \mathcal{O}\left(M_{W, Z, t}\right)$, the one-loop FCNC $b \rightarrow s(d)$ processes with $W, Z$, and top quark propagators, such as the penguin loop, can be properly described by effective point-like vertices (Figure 1.3). Consequently the effective Hamiltonian governing the one loop FCNC transitions is described by

$$
\mathcal{H}_{\text {eff }}=-\frac{4 G_{F}}{\sqrt{2}} V_{t b} V_{t q}^{*} \sum_{i} \mathcal{C}_{i}(\mu) \mathcal{O}_{i}
$$

where $\mathcal{O}_{i}$ 's are operators for the point-like vertices and $V_{t b} V_{t q}^{*}$ are elements of the CKM matrix with $q=s, d$, and the $C_{i}$ 's represent the corresponding Willson coefficient and contain the relevant short-distance physics from scale higher than $\mu$.

The operator for the $b \rightarrow s \gamma$ penguin vertex $\mathcal{O}_{7}$ is given by

$$
\mathcal{O}_{7}=\frac{e}{8 \pi^{2}} m_{b} \bar{s}_{\alpha} \sigma^{\mu \nu}\left(1+\gamma_{5}\right) b_{\alpha} F_{\mu \nu} .
$$

The coefficient $\left(\mathcal{C}_{i}\right)$ is firstly calculated with $\mu=\mathcal{O}\left(M_{W}\right)$, then evoluted to $\mu=$ $\mathcal{O}\left(m_{b}\right)$. Because of the asymptotic freedom the QCD, $\mathcal{C}_{i}(\mu)$ can be calculated in perturbation theory as long as $\mu$ is not too small. It is known that there is large correction $(\times 2)$ for the decay rate by the higher order processes. The leading-order (LO) correction corresponds to the two-loop diagrams, and the next-to-leading-order correction corresponds to the three-loop diagrams. 

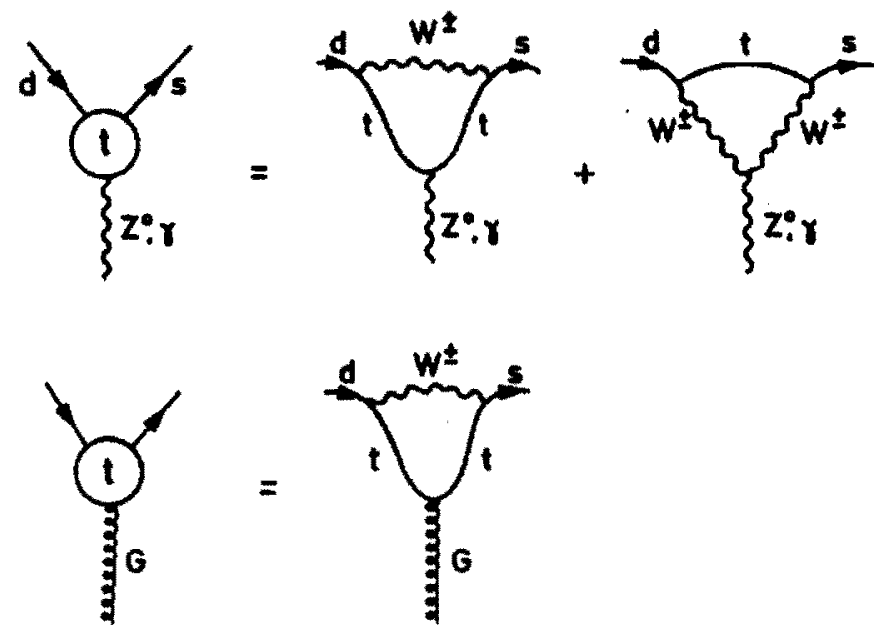

Figure 1.3: Penguin vertices resolved in terms of point-like vertices.

In calculating $B\left(B \rightarrow X_{s} \gamma\right)$, the measured semileptonic rate $B\left(B \rightarrow X_{c} e \bar{\nu}\right)$ is usually introduced in order to reduce the uncertainties due to the CKM matrix elements and the $m_{b}^{5}$. The branching fraction is written by

$$
B\left(B \rightarrow X_{s} \gamma\right)=\frac{\Gamma(b \rightarrow s \gamma)}{\Gamma(b \rightarrow c e \bar{\nu})} B\left(B \rightarrow X_{c} e \bar{\nu}\right)
$$

The LO calculation of the $B\left(B \rightarrow X_{s} \gamma\right)$ which was completed in 1993 [20] is

$$
B\left(B \rightarrow X_{s} \gamma\right)=(2.8 \pm 0.8) \times 10^{-4}
$$

where the uncertainty is caused by the scale $(\mu)$ uncertainty. The NLO calculation is completed in 1998 [21], and gives

$$
B\left(B \rightarrow X_{a} \gamma\right)=(3.28 \pm 0.30) \times 10^{-4} .
$$

The NLO correction does not change the mean value of the decay rate itself so much, but significantly reduce its uncertainty $(30 \% \rightarrow 10 \%)$.

The inclusive $b \rightarrow X_{s} \gamma$ decay is theoretically clean and the rate can be predicted with $10 \%$ precision. However, it is difficult to measure the rate experimentally, especially for the hadron collider environment. On the other hand, exclusive radiative decays have a distinctive signature which makes them experimentally accessible. The ratio of the rates for the exclusive $\left(B \rightarrow K^{*} \gamma\right)$ and inclusive decays are given by [21],

$$
R_{K^{*}}=\frac{B\left(B \rightarrow K^{*} \gamma\right)}{B\left(B \rightarrow X_{s} \gamma\right)}=4\left(\frac{m_{B}}{m_{b}}\right)^{2}\left(1-\frac{m_{K^{*}}^{2}}{m_{B}}\right)^{3}\left|T_{1}\left(q^{2}=0\right)\right|^{2}
$$


where $T_{1}\left(q^{2}=0\right)$ is the form factor for $B \rightarrow K^{*}$. The form factor is estimated with several methods such as lattice QCD, and $R_{K^{*}}$ is calculated to be $0.1 \sim 0.2$. It is in good agreement with the CLEO result of $R_{K^{*}}=(18 \pm 6) \%$. The bracing fraction for the exclusive radiative decay of the $B_{s}$ meson, $B_{s} \rightarrow \phi \gamma$ is calculated to be similar as that for $B \rightarrow K^{*} \gamma$. The bracing fraction for the exclusive radiative decay of the $b$-baryon, $\Lambda_{b} \rightarrow \Lambda \gamma$ is calculated to be $0.2-5 \times 10^{-5}[22,23]$.

\subsection{Radiative $B$ Decay Beyond the Standard Model}

Rare $B$ decays provide several probes of new physics beyond the Standard Model. Since the more massive the particles participating in the loop are, the higher the branching fraction for the radiative decays, the existence of non-SM heavy charged particles, such as charged Higgs and squarks, could affect the branching fraction. In addition, direct CP-violating effects could be enhanced by processes beyond the Standard Model. Measurements of such processes, therefore, constitute low energy probes for physics beyond the SM.

For example, the reach of radiative $B$ decays in probing the supersymmetric parameter space is comparable with that of direct search at high energy colliders [24]. Figure 1.4 shows the $B(b \rightarrow s \gamma)$ in a supergravity model as a function of the charged Higgs mass for $\tan \beta=2$ (top) and $\tan \beta=30$ (bottom). Each solid line shows the branching fraction in the two Higgs doublet model. Each dashed line shows the branching fraction in the SM. Dotted lines denote the upper and lower bounds on the branching fraction given by CLEO.

Since the Standard Model predicts that the CP-violating effects in the radiative $B$ decay is small $(\sim 1 \%)$, the search for CP-violating effect in rare $B$ decay is sensitive to the new physics. For example, if there are new physics contribution to the radiative $B$ decay, the $\mathcal{C}_{7} \mathcal{O}_{7}$ operator structure of SM can become $\mathcal{C}_{7} \mathcal{O}_{7}+\mathcal{C}_{7}^{\prime} \mathcal{O}_{7}^{\prime}$ where $\mathcal{O}_{7}^{\prime}$ has different chirality to $\mathcal{O}_{7}$ and given by

$$
\mathcal{O}_{7 \gamma}^{\prime}=\frac{e}{8 \pi^{2}} m_{b} \bar{s}_{\alpha} \sigma^{\mu \nu}\left(1-\gamma_{5}\right) b_{\alpha} F_{\mu \nu}
$$

This leads to large CP-violating effect compared to the SM $[26,27]$.

- Mixing induced $\mathrm{CP}$ violation

Interference between $\mathcal{O}_{7}$ and $\mathcal{O}_{7}^{\prime}$ causes $\mathrm{CP}$ violations. For $B \rightarrow M \gamma$ where $M$ 

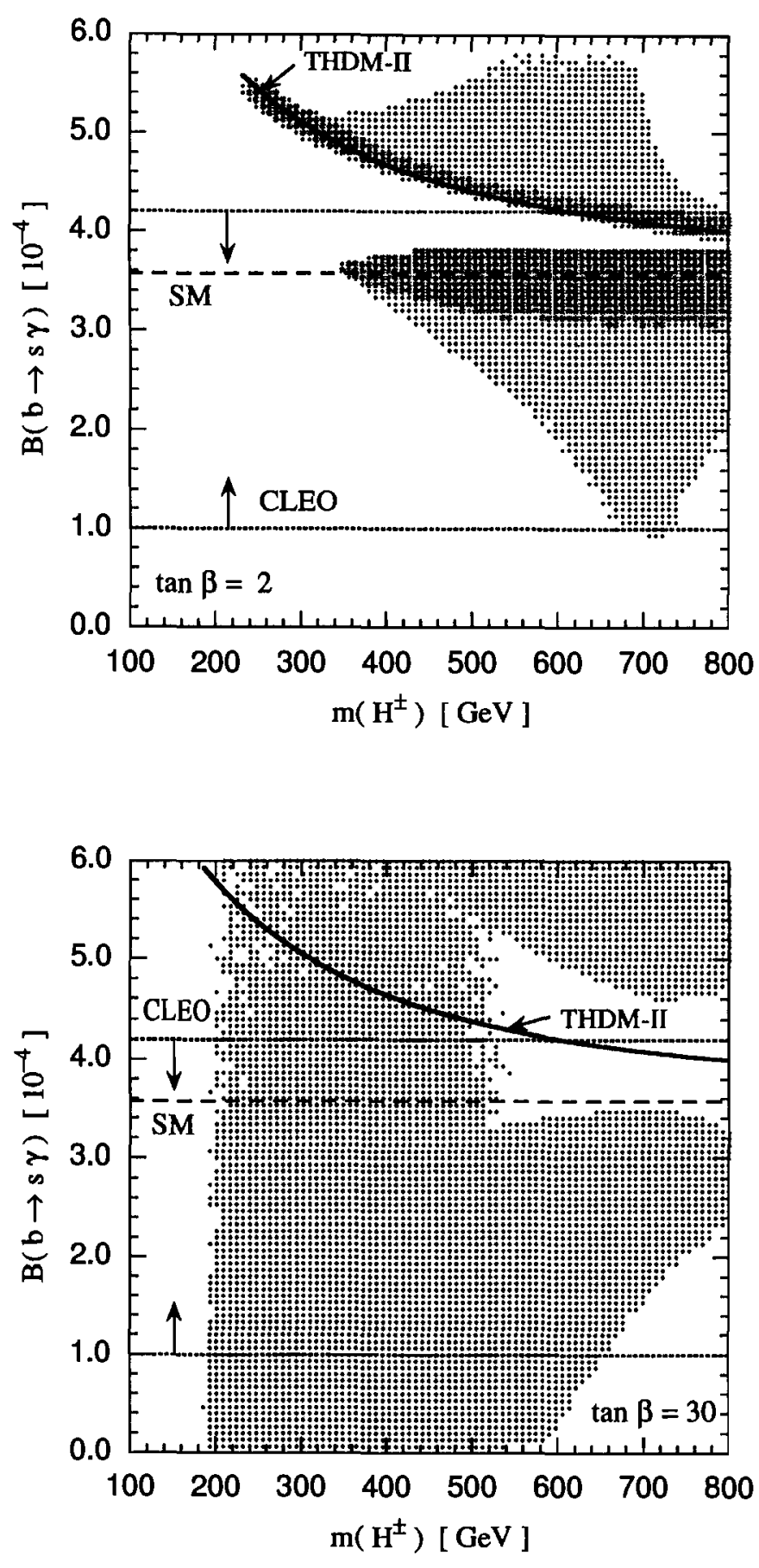

Figure 1.4: $B(b \rightarrow s \gamma)$ in the supergravity model as a function of the charged Higgs mass for $\tan \beta=2$ (top) and $\tan \beta=30$ (bottom). Each solid line shows the branching fraction in the two Higgs doublet model. Each dashed line shows the branching fraction in the SM. Dotted lines denote the upper and lower bounds on the branching fraction given by CLEO. 
is a CP eigenstate such as $K^{* 0} \rightarrow K^{0} \pi^{0}$ and $\phi \rightarrow K^{+} K^{-}$, one obtains a mixing dependent asymmetry:

$$
A_{m i x} \equiv \frac{\Gamma(\bar{B} \rightarrow f)-\Gamma(B \rightarrow f)}{\Gamma(\bar{B} \rightarrow f)+\Gamma(B \rightarrow f)} \propto \frac{\left|\mathcal{C}_{7} \mathcal{C}_{7}^{\prime}\right|}{\left|\mathcal{C}_{7}\right|^{2}+\left|\mathcal{C}_{7}^{\prime}\right|^{2}} \sin \left(\phi_{B}-\phi-\phi^{\prime}\right)
$$

where $\phi\left({ }^{\prime}\right)$ are the weak passes for $\mathcal{C}_{7}^{\prime}$ and $\phi_{B}$ is weak phase from the $B$ mixing. The asymmetry can be $80 \%$ in some models such as Left-Right Symmetry Model (LRSM) through the effect of squark mixing $[26,27]$.

- $\Lambda$ polarization in $\Lambda_{b} \rightarrow \Lambda \gamma$

A measurement of $\Lambda$ polarization $\left(\alpha_{\Lambda}\right)$ is interesting in the $\Lambda_{b} \rightarrow \Lambda \gamma$ decay. The $\alpha_{\Lambda}$ is defined by

$$
\frac{d \Gamma}{d \cos \theta} \propto 1-\alpha_{\Lambda} \cos \theta
$$

where $\theta$ is the angle between the direction of the momentum of $\Lambda$ in the $\Lambda_{b}$ rest frame and direction of the $\Lambda$ polarization in the $\Lambda$ rest frame. The SM predicts the $\alpha_{\Lambda}$ is exactly 1 except the small correction $(2 \%)$ due to the long distance contribution and the mass of the $s$-quark $\left(\mathcal{O}\left(\left(\frac{m_{a}}{m_{b}}\right)^{2}\right)\right)$. On the other hand, some of the physics models give different expectations and $\alpha_{\Lambda}$ is given by

$$
\alpha_{\Lambda}=\frac{\left|\mathcal{C}_{7}\right|^{2}-\left|\mathcal{C}_{7}^{\prime}\right|^{2}}{\left|\mathcal{C}_{7}\right|^{2}+\left|\mathcal{C}_{7}^{\prime}\right|^{2}}
$$

The effect can reach $50 \%$ in some cases.

\subsection{B Production at CDF}

In this section we discuss the production of $B$ hadrons in $p \bar{p}$ collision at a center of mass energy of $1.8 \mathrm{TeV}$. The production mechanism of the $B$ hadron is a good stage to test the QCD.

\subsection{1 b-Quark Production}

The $b \bar{b}$ production in the $p \bar{p}$ collision is dominated by QCD processes. Figure 1.5 shows the $\mathcal{O}\left(\alpha_{s}^{2}\right)$ diagrams. In these diagrams, a $b \bar{b}$ pair is produced back-to-back in the rest frame of the initial state partons. Figure 1.6 shows the $\mathcal{O}\left(\alpha_{s}^{3}\right)$ diagrams. In the higher 

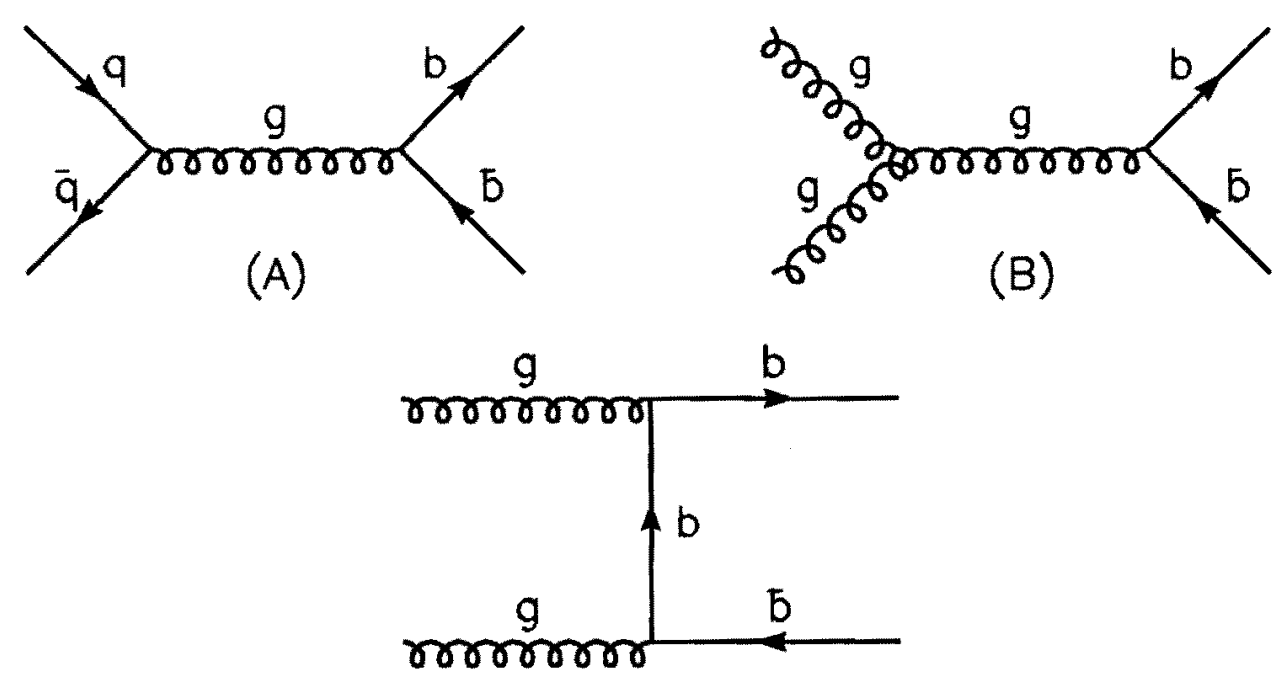

(C)

Figure 1.5: Leading order Feynman diagrams depicting $b$-quark production by quark-antiquark annihilation ( $\mathrm{A}$ ), and gluon-gluon fusion (B and $\mathrm{C}$ ).

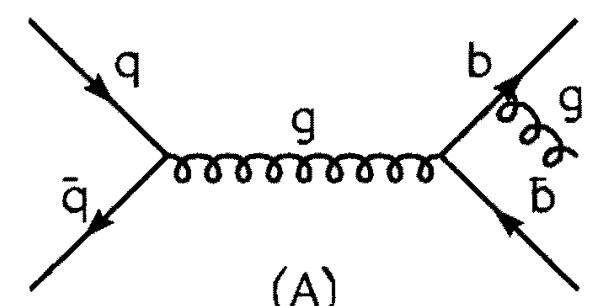

(A)

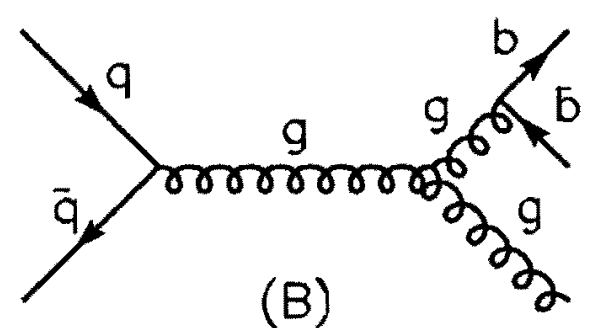

(B)

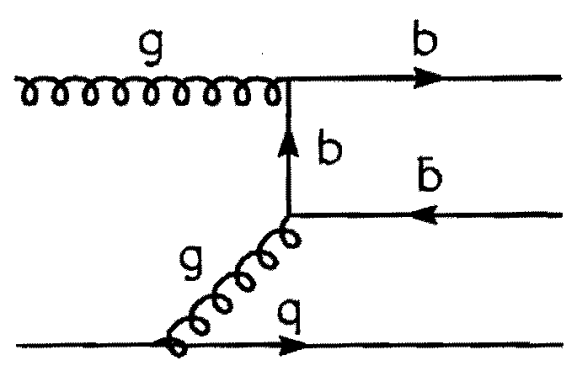

(C)

Figure 1.6: Next-to-leading order Feynman diagrams depicting b-quark production by gluon radiation (A), gluon splitting (B), and flavor excitation (C). 


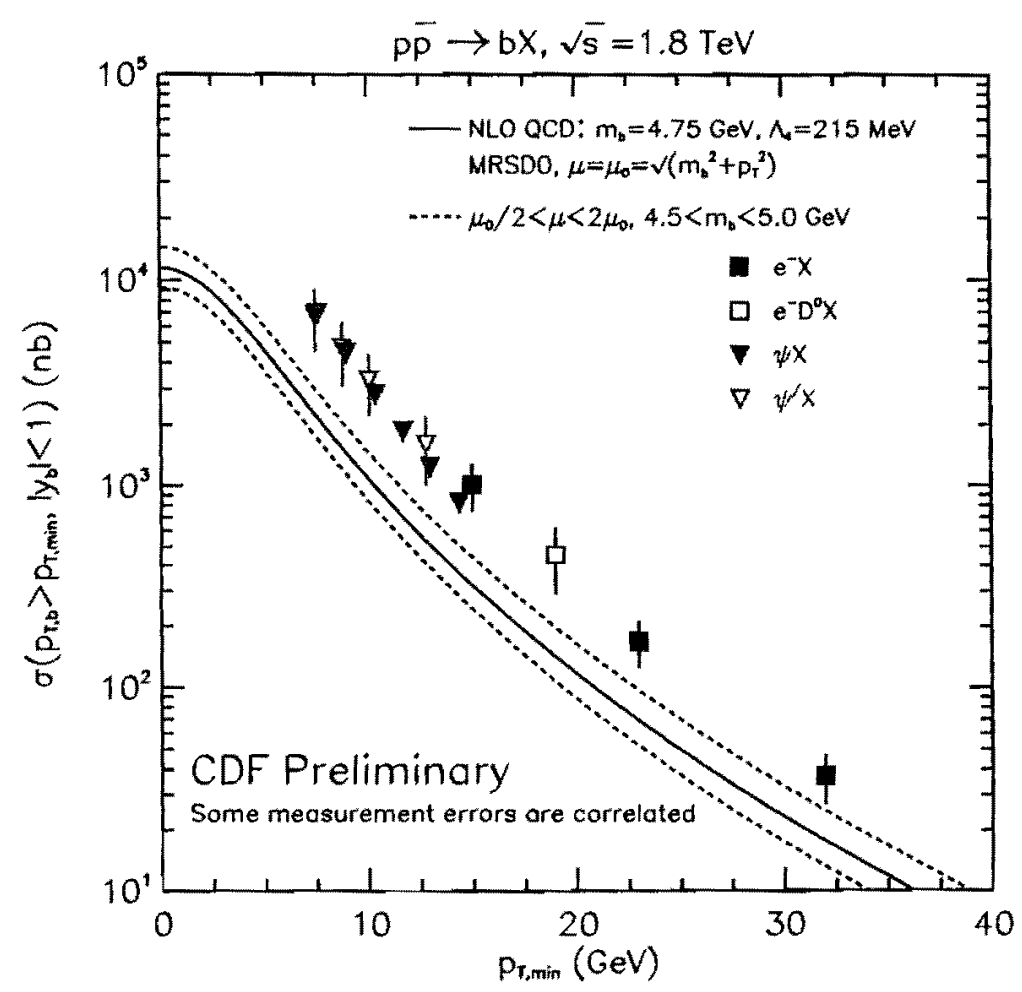

Figure 1.7: The $b$-quark production cross section at the CDF.

order diagrams the $b \bar{b}$ pair is not necessary to be back-to-back. In the gluon splitting diagram (B), they have almost the same direction.

Figure 1.7 shows the measured integrated $b$-quark production cross section as a function of its momentum transverse to the $p \bar{p}$ beamline $\left(p_{T}\right)$ at the CDF by using several decay channels. The typical $b$-quark production cross section is $\sim 5 \mu \mathrm{b}$. Figure 1.7 also shows the theoretical prediction (solid line) based on a next-to-leading order QCD calculation [28] using the MRSD0 parton distribution functions [29] and a renormalization scale of $\mu=\mu_{0} \equiv \sqrt{m_{b}^{2}+p_{T}^{2}}$ with $m_{b}=4.75 \mathrm{GeV} / c^{2}$ for the mass of the $b$ quark and $p_{T}$ for its transverse momentum. Dashed lines correspond to the theoretical uncertainty by changing $1 / 2 \mu_{0}<\mu<\mu_{0}$ and $4.5<m_{b}<5.0 \mathrm{GeV} / c^{2}$. The measured cross section at $\mathrm{CDF}$ is approximately three times larger than the QCD calculation.

\subsection{2 $b$-Quark Fragmentation}

The $b$ quarks are observed as colorless hadrons such as $\bar{B}_{d}, \bar{B}_{s}$, and $\Lambda_{b}$. The $b$ quarks fragment in the $B$ hadrons through low $Q^{2}$ QCD process. Figure 1.8 shows a schematic diagram of the fragmentation process. The momentum fraction which the final state $B$ hadron carries out, $D=p_{T}(B) / p_{T}(b)$, is parametrized by Peterson [30] as 


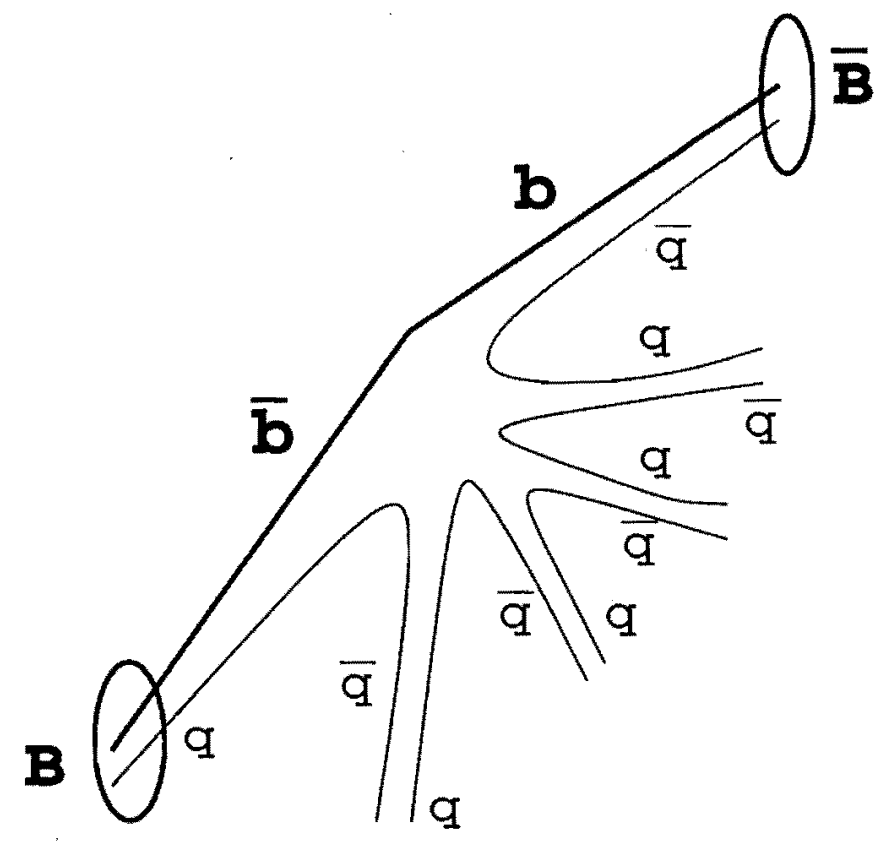

Figure 1.8: A schematic view of the fragmentation of $b$-quark into $B$ meson.

$$
\frac{d N}{d D} \sim \frac{1}{z\left(1-\frac{1}{D}-\frac{\epsilon}{1-D}\right)^{2}}
$$

where $\epsilon$ is the Peterson parameter related to the ratio $\left(m_{q} / m_{b}\right)^{2}$ with $m_{q}$ denoting the mass of the spectator quark (diquark) for $B$ meson (hadron). Thus the fragmentation can differ between the $B$-hadron species. Figure 1.9 shows the Peterson fragmentation function with $\epsilon=0.004,0.006$, and 0.008 .

Figure 1.10 shows the measured $B$ meson production differential cross section as a function of the $p_{T}$. The solid line shows the theoretical prediction with $\epsilon=0.006$ and dashed lines are corresponding the theoretical uncertainty by changing $1 / 2 \mu_{0}<\mu<\mu_{0}$, $4.5<m_{b}<5.0 \mathrm{GeV} / c^{2}, 0.004<\epsilon<0.008$. The measured cross section is three times larger than the QCD calculation.

\subsection{Summary}

Radiative $B$ decay occurs through the penguin diagram. The exclusive decays of $B^{0, \pm} \rightarrow$ $K^{*} \gamma$ were observed at CLEO. However, the $B_{s}$ and $\Lambda_{b}$ radiative decays have not been observed yet. Since the $B$ factories at the $\Upsilon(4 S)$ resonance can not produce the heavier $B$-hadrons such as $B_{s}$ and $\Lambda_{b}$, the measurements of the $B_{s}$ and $\Lambda_{b}$ decays are unique at 


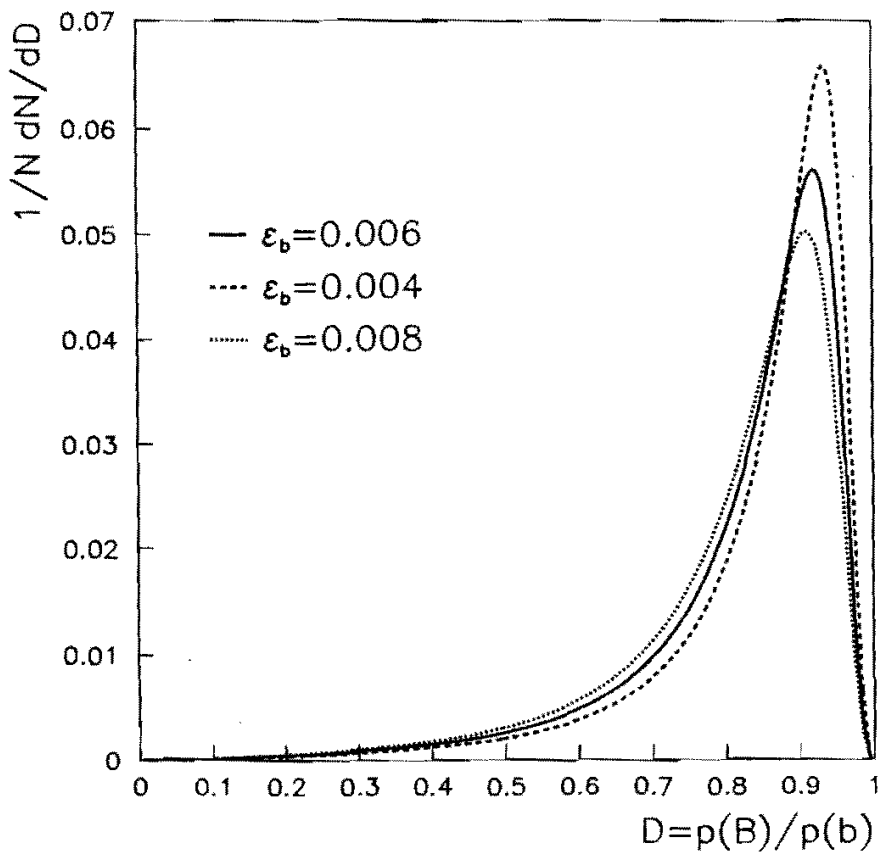

Figure 1.9: The Peterson fragmentation functions for three different $\epsilon$ 's.

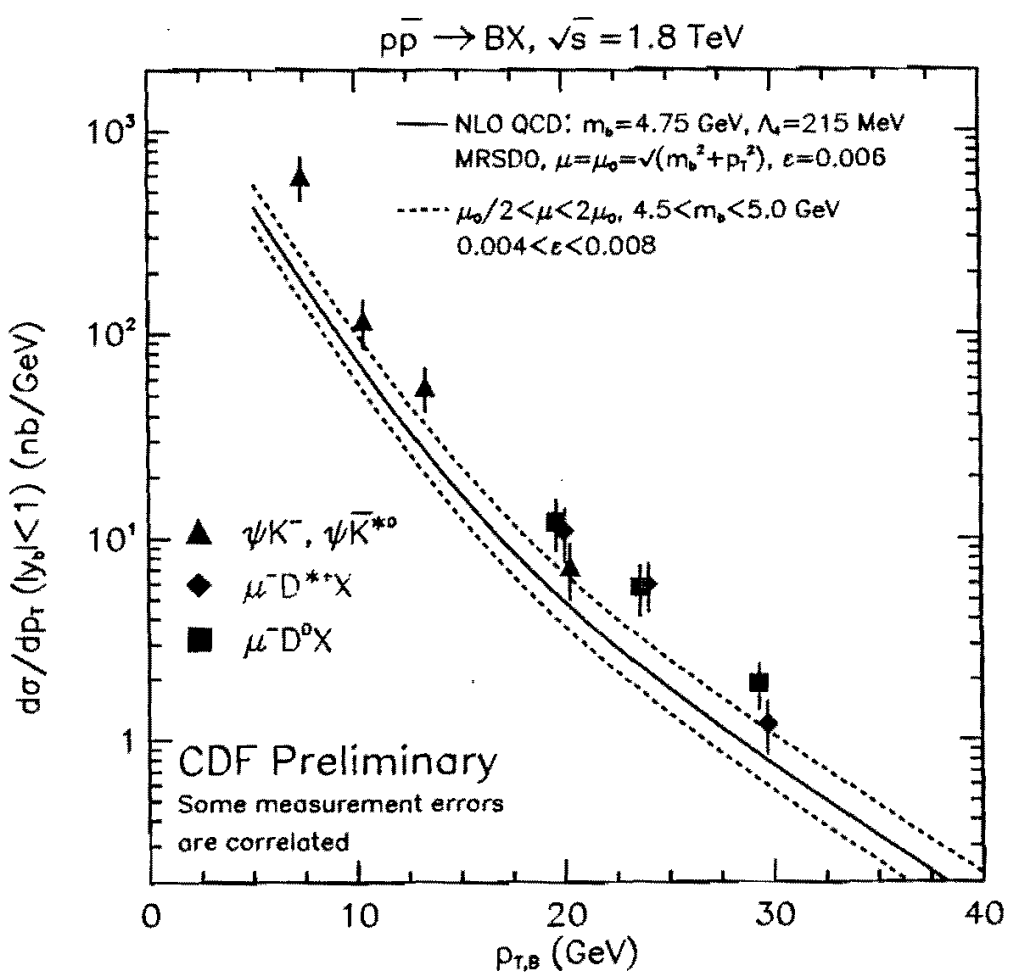

Figure 1.10: The $B$-meson production cross section at the CDF. 
the high energy collider experiments.

Measurement the radiative $B$ decay is sensitive to the CKM matrix elements $V_{t s}$ and $V_{t d}$. The ratio of the CKM matrix elements $\left|V_{t d}\right| /\left|V_{t s}\right|$ is extracted from the ratio of the branching fractions between the $B_{s} \rightarrow K^{* 0} \gamma$ and $B_{s} \rightarrow \phi \gamma$. Since the $\phi$ and $K^{*}$ mesons have narrow width, this measurement is interesting. In this analysis the $B$ mass resolution is critical to separate the $B_{s} \rightarrow K^{* 0} \gamma$ and $B_{d} \rightarrow K^{* 0} \gamma$ decays.

The penguin diagram is also sensitive to the presence of new heavy particles beyond the standard model. There are two interesting measurements: 1) the mixing induced CP violation in the $\left.B_{s} \rightarrow \phi\left(\rightarrow K^{+} K^{-}\right) \gamma ; 2\right)$ the polarization of the $\Lambda$ in the $\Lambda_{b} \rightarrow \Lambda \gamma$ decay. These two measurements are unavailable at the $B$-factories at the $\Upsilon(4 S)$ resonances.

This thesis is organized as follows. In Chapter 2 we describe the experimental apparatus, i.e., the Tevatron collider and the CDF detector. In Chapter 3 we describe the event selection criteria of the radiative $B$ decays. In Chapter 4 we describe the calculation of the efficiency for the radiative $B$ signal which is necessary for measuring the branching fraction. In Chapter 5 we describe the estimation of the systematic uncertainty for the branching fraction measurement. In Chapter 6 we describe the results of the branching fraction measurement. Finally, Chapter 7 concludes the thesis. 


\section{Chapter 2}

\section{Experimental Apparatus}

\subsection{Accelerator Overview}

The Fermilab accelerator complex is shown schematically in Figure 2.1. It consists of six different accelerator systems: Pre-accelerator, Linac, Booster, Main Ring, Antiproton Storage Ring, and Tevatron.

The Pre-accelerator is the first of the accelerators. It consists of the source of the negatively-charged hydrogen ions $\left(\mathrm{H}^{-}\right)$housed inside a Cockcroft-Walton which is charged to a potential of $-750 \mathrm{kV}$. So the $H^{-}$is accelerated to an energy of $750 \mathrm{keV}$. The Linear Accelerator (Linac) is the next level of acceleration for the $\mathrm{H}^{-}$. It takes the ions with an energy of $750 \mathrm{keV}$ and accelerates them to an energy of $400 \mathrm{MeV}$. The Booster is the next level of acceleration, taking the $400 \mathrm{MeV}$ negative hydrogen ions, stripping the electrons off leaving only the proton core, and accelerating the protons to $8 \mathrm{GeV}$. The Booster is the first synchrotron in the acceleration chain. It consists of a series of magnets arranged around a 75 meter radius circle, with $17 \mathrm{RF}$ cavities interspersed. The Main Ring is a circular synchrotron of radius 1000 meters. It can accelerate protons from the Booster from $8 \mathrm{GeV}$ to either $120 \mathrm{GeV}$ or $150 \mathrm{GeV}$, depending on their destination. As well as accepting protons from Booster, the Main Ring can accept antiprotons from the antiproton source at $8 \mathrm{GeV}$ and accelerate them to $150 \mathrm{GeV}$. Once the proton or antiproton beam in Main Ring is accelerated to $150 \mathrm{GeV}$, it can be sent to the Tevatron. The proton beam that is accelerated to $120 \mathrm{GeV}$ can be sent to the Antiproton source.

The $120 \mathrm{GeV}$ protons which are stripped off the Main Ring hit a tungsten target and produce antiprotons. These antiprotons are directed to the Debuncher. The Debuncher is one of the two synchrotrons that make up the antiproton source. Its primary purpose is to reduce the momentum spread of the antiprotons by rotating the bunches. There are also beam cooling systems that act to reduce the oscillations in the plane perpendicular to the orbit (transverse plane) as well as reducing the momentum spread of the antiprotons. The antiproton is then transferred to the Accumulator. The Accumulator is the second 
synchrotron of the antiproton source. It is the storage ring for the antiprotons: all of the antiprotons made are stored here at $8 \mathrm{GeV}$ and cooled until needed. Under typical condition the stack of antiprotons can be built at a rate of $\sim 4 \times 10^{10}$ antiprotons per hour.

The Tevatron is a circular synchrotron of radius 1000 meters that has the same basic layout and shares the same radiation enclosure as the Main Ring. The RF section of the accelerator consists of 8 accelerating cavities. The Tevatron can accept both protons and antiprotons from Main Ring and accelerate them from $150 \mathrm{GeV}$ to $900 \mathrm{GeV}$. Six bunches of the protons and antiprotons are transferred into the Tevatron ring. Thus there are six regions where the proton and antiproton bunches can be made to collide each other, and the collisions occur every $3.5 \mu \mathrm{s}$. The Tevatron ring is divided up into 6 sections, labeled A through $\mathrm{F}$, and each section is divided up into 5 subsections (A0 trough A4). The Collider Detector at Fermilab (CDF) and D0 detector are located at the $\mathrm{B} 0$ and $\mathrm{D} 0$ interaction region, respectively.

Typical numbers of the protons and antiprotons are $2.0 \times 10^{11}$ and $5.5 \times 10^{10}$ per bunch, respectively. Low $\beta$ quadrupole magnets which are located immediately upstream of the CDF detector reduce the beam size the, and the dimensions of each bunch are $\sim 30 \mathrm{~cm}$ along the beamline and $\sim 30 \mu \mathrm{m}$ in the transverse direction at the nominal interaction point. Typical instantaneous luminosity is $1.6 \times 10^{31} \mathrm{~cm}^{-2} \mathrm{~s}^{-1}$, and its lifetime is $\sim 10$ hours.

Figure 2.2 shows the integrated luminosity delivered to and accumulated by the CDF detector during the years $1992-1996$ (Run I). The data taking efficiency of the CDF detector is $\sim 75 \%$. We collected data within three sub-periods; Oct. 1992 - Jun. 1993 (Run IA), Feb. 1994 - Jul. 1995 (Run IB), Nov. 1995 - Feb. 1996 (Run IC). The data used in this analysis was collected during the Run IB.

\subsection{Detector Overview}

The CDF Detector is a multi-purpose detector designed to detect the particles produced in $p \bar{p}$ collisions. Figure 2.3 shows an isometric view of the CDF detector. The detector consists of a $5000 \mathrm{t}$ central detector which made up of the solenoidal magnet, steel yoke, tracking detectors, electromagnetic and hadron calorimeters, and muon chambers, and two identical forward/backward detectors consisting of segmented time-of-flight counters, electromagnetic and hadron calorimeters, and muon toroidal spectrometers [31].

Figure 2.4 shows a schematic of the $r z$-view of the detector. In CDF, the positive 


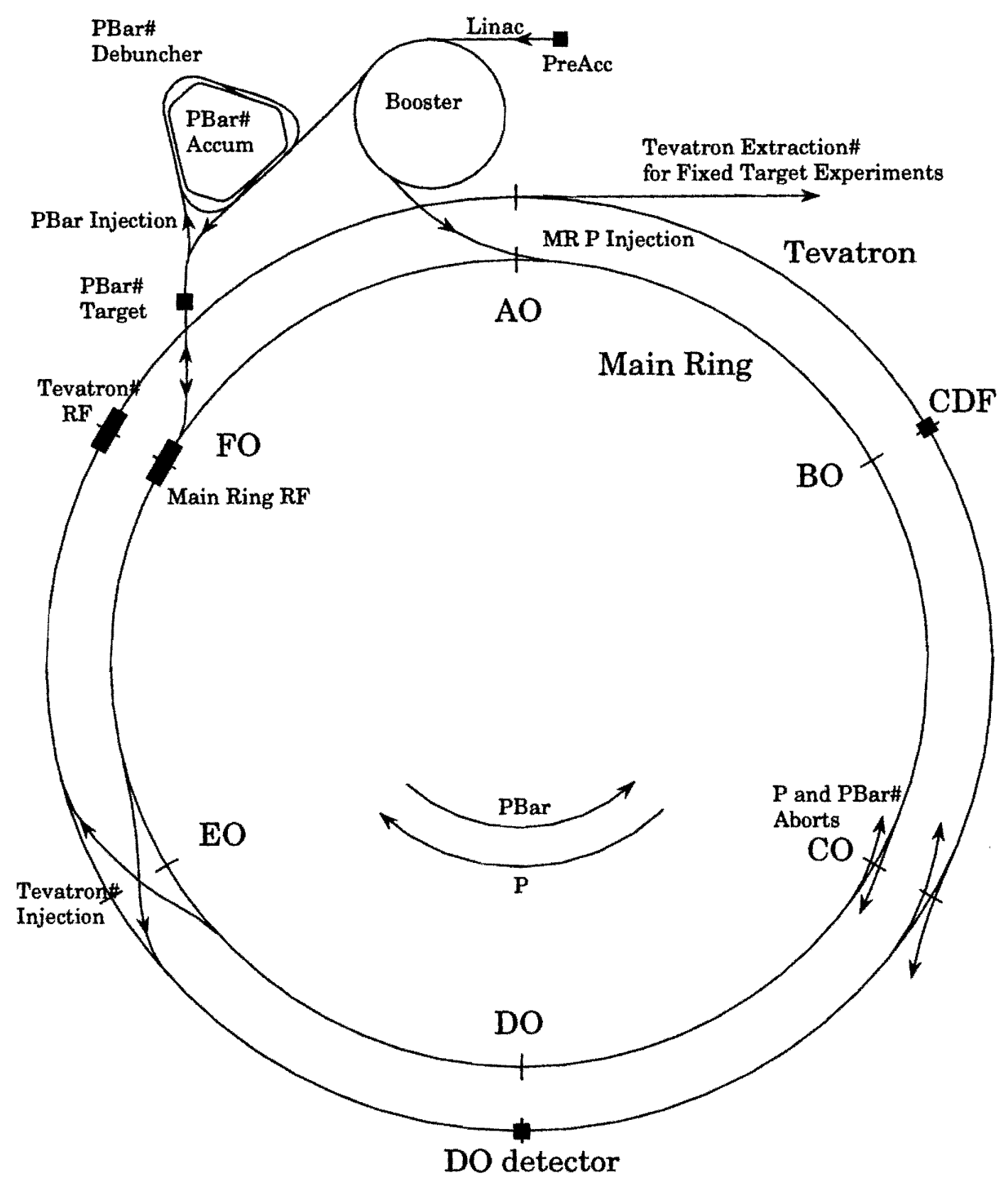

Figure 2.1: A schematic view of Fermilab accelerator complex. 


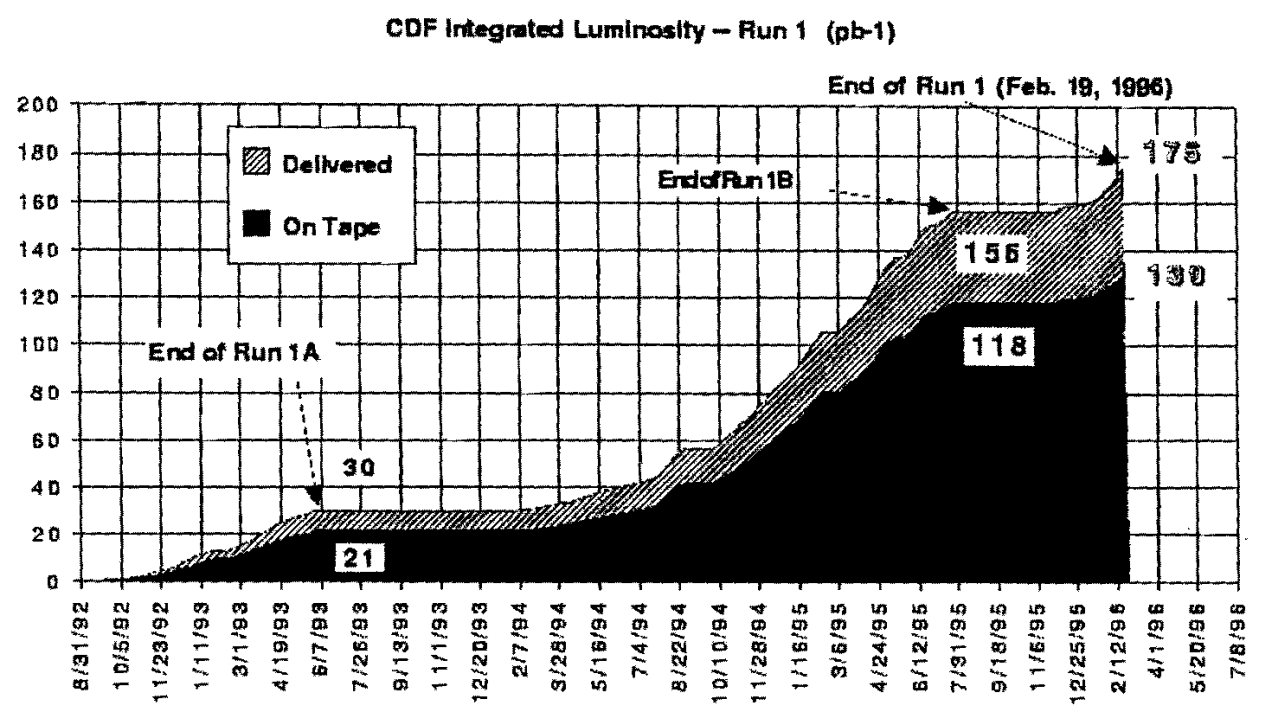

Figure 2.2: The integrated luminosity delivered to the $\mathrm{B} 0$ collision point and the corresponding luminosity collected by CDF.

$z$ is defined along the proton beam direction, the $r$ is transverse to the $z$ direction, $\theta$ is the polar angle, and $\phi$ is the azimuthal angle with respect to the Tevatron plane. The radial distance from the $z$ axis is denoted by $r$. The pseudorapidity $\eta$ is defined as $\eta=-\operatorname{In} \tan (\theta / 2)$. The origin of coordinates is the physical center of the detector, which is the nominal interaction point.

The relevant detector components for this analysis to observe the radiative decays are the central charged-particle tracking system and the central calorimeters. In the following we describe an outline of the detectors.

\subsection{Tracking Detector}

There are three separate tracking devices in the CDF. Immediately outside the beam pipe there is the silicon vertex detector (SVX) [32]. The vertex time projection chamber (VTX) [33] surrounds the SVX, the cylindrical central drift chamber (CTC) [34] surrounds the VTX.

The tracking detectors are located inside a $1.4 \mathrm{~T}$ solenoidal magnetic field, and their main purposes are: (1) to measure momentum of charged particles and their generation point; (2) to measure the primary vertex of the $p \bar{p}$ interaction point; (3) to measure the ionizing energy loss in the CTC $(d E / d x)$ for particle identification; (4) to serve as photon radiators. 


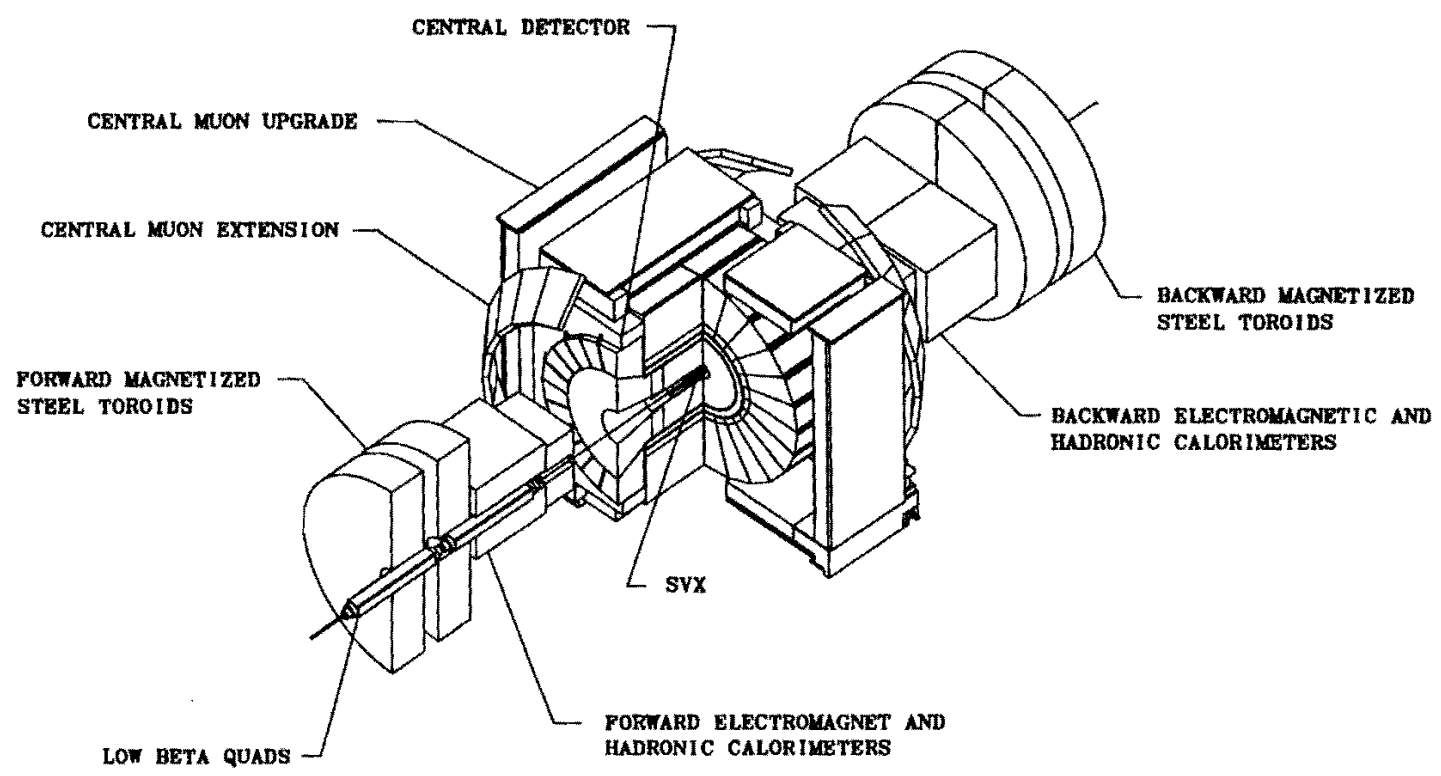

Figure 2.3: An isometric view of the CDF detector.

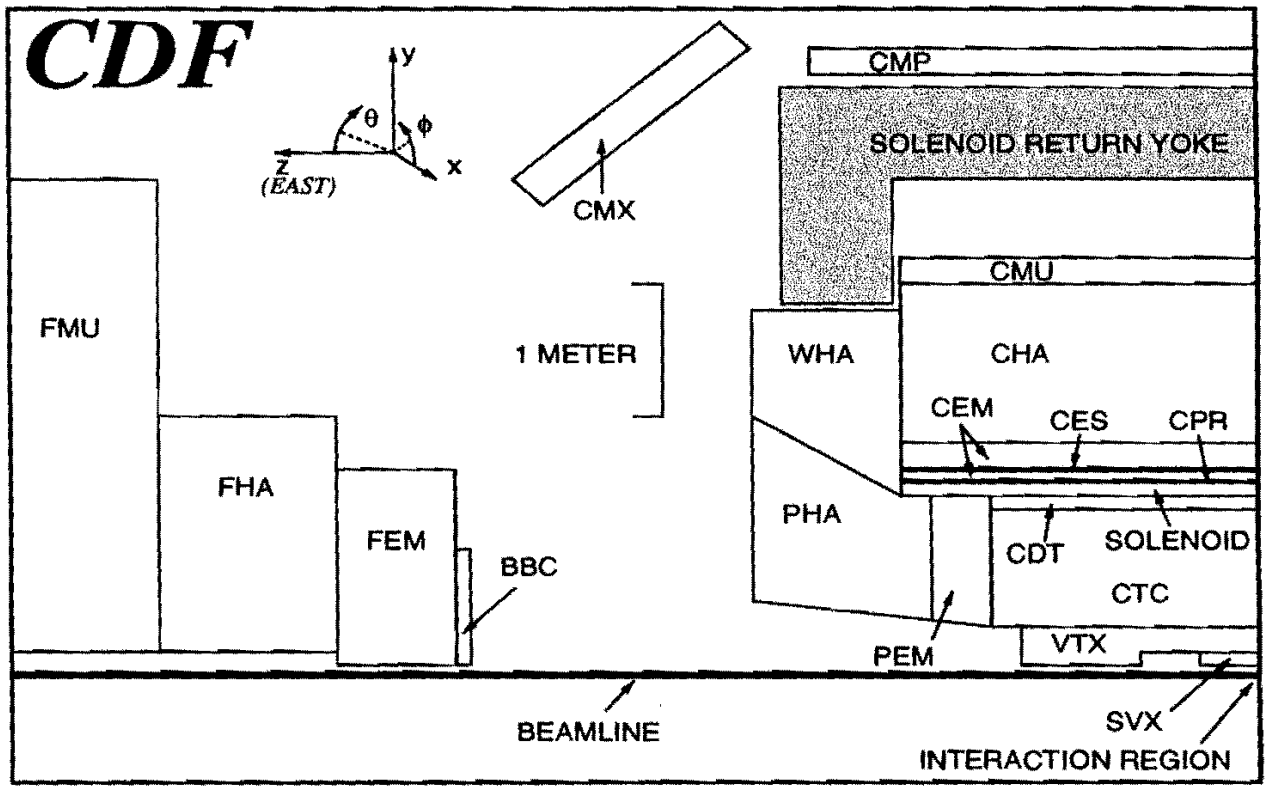

Figure 2.4: A side-view cross section of the CDF detector. 


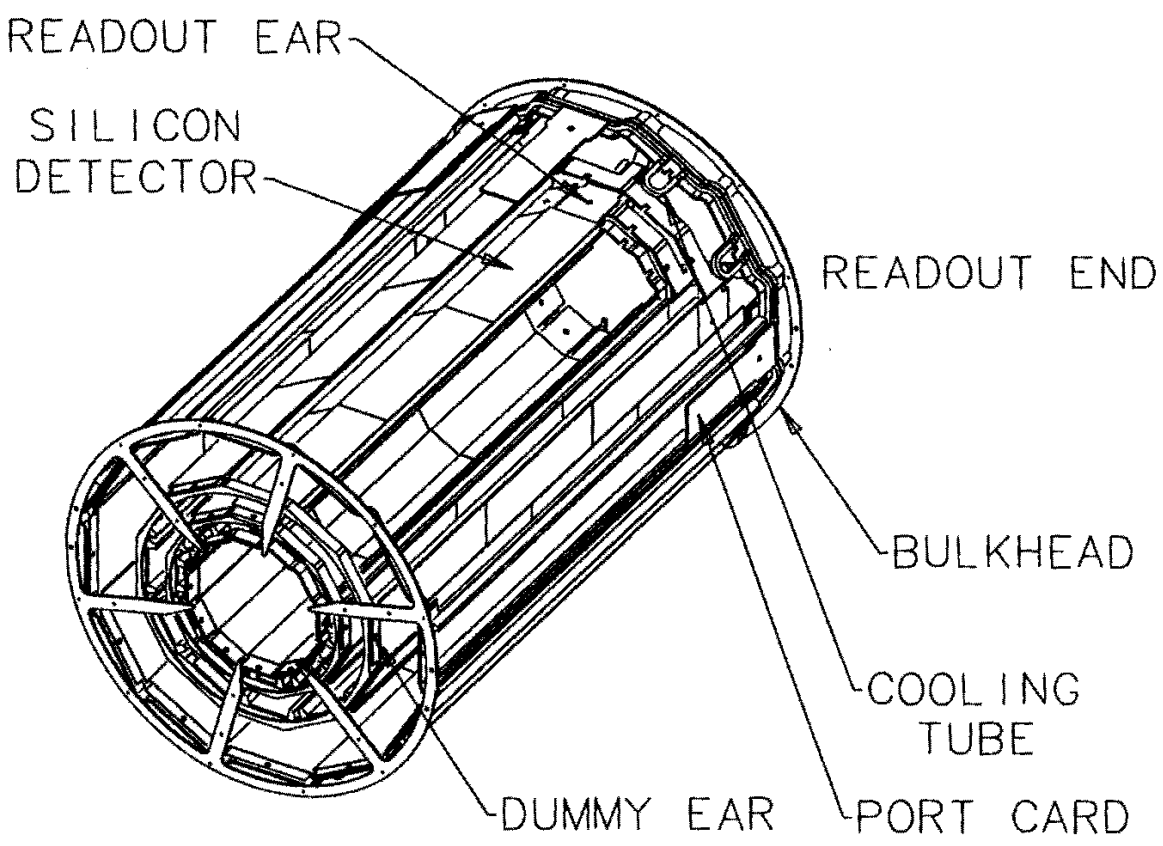

Figure 2.5: An isometric view of the SVX.

\subsubsection{Silicon Vertex Detector (SVX)}

Figure 2.5 shows a schematics of one of the two barrels of the SVX detector which covers $z>0$. The SVX covers the region $|z|<25.55 \mathrm{~cm}$, consisting of four concentrical cylindrical layers of single sided silicon detectors which are located at radii $2.9,4.3,5.7$ and $7.9 \mathrm{~cm}$.

Each SVX layer consists of twelve flat ladders that cover $30^{\circ}$ in $\phi$. Figure 2.6 shows a schematics of the SVX ladder. Each ladder has three single sided silicon strip detectors with $300 \mu \mathrm{m}$ thick n-type semiconductor and 55-60 $\mu \mathrm{m}$ pitch p-type strips along the direction of the $z$ axis. The three silicon detectors in a ladder are electrically bonded together and electric signal is read by readout chips which are mounted on the "readout ear". The total number of read out channels is 46080 .

Since the SVX has strips along the $z$ direction, it provides information on the travel path of charged particles only in the transverse plane. The average position resolution is $\sim 12 \mu \mathrm{m}$ for each layer. Since the $p \bar{p}$ collisions are distributed in a rather long region along the beamline (Gaussian distribution with $\sigma \sim 30 \mathrm{~cm}$ ), only $\sim 60 \%$ of them occur in the SVX fiducial volume. Its precise tracking close to the beamline can help us to distinguish the tracks of $B$ decay products from a plethora of tracks originating from the $p \bar{p}$ interaction point. 


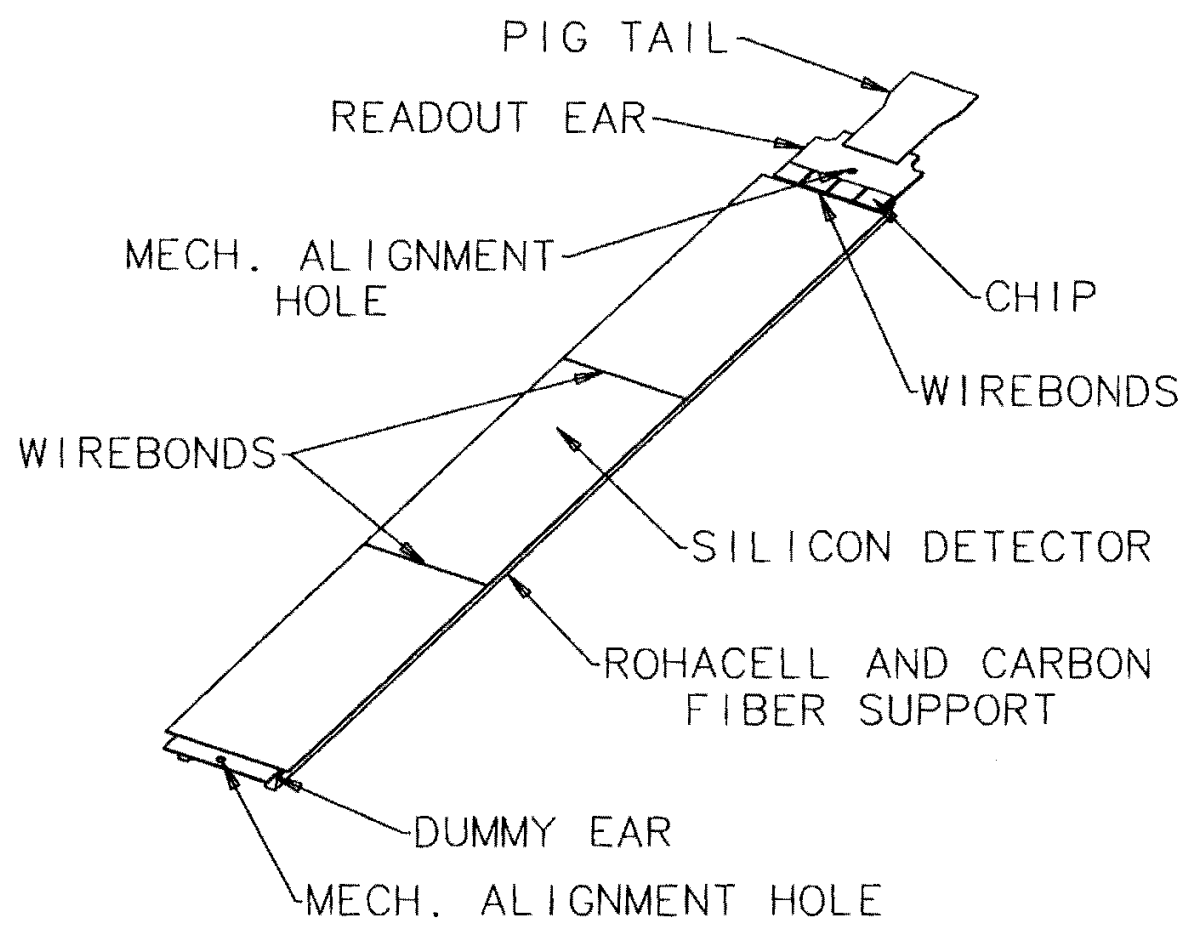

Figure 2.6: An isometric view of the SVX ladder.

\subsubsection{Vertex Time Projection Chamber (VTX)}

The vertex time projection chamber (VTX) [33] surrounds the SVX and it reconstructs tracks in the $r-z$ plane. Figure 2.7 shows a schematic of the VTX It occupies the region $|z|<136 \mathrm{~cm}$ with 28 drift modules which have an outer radius of $22 \mathrm{~cm}$ and are connected together along the $z$ axis. Its primary task in this analysis is to locate the $p \bar{p}$ interaction point along the $z$ axis with resolution around $1-2 \mathrm{~mm}$ depending on the number of charged tracks going through the VTX.
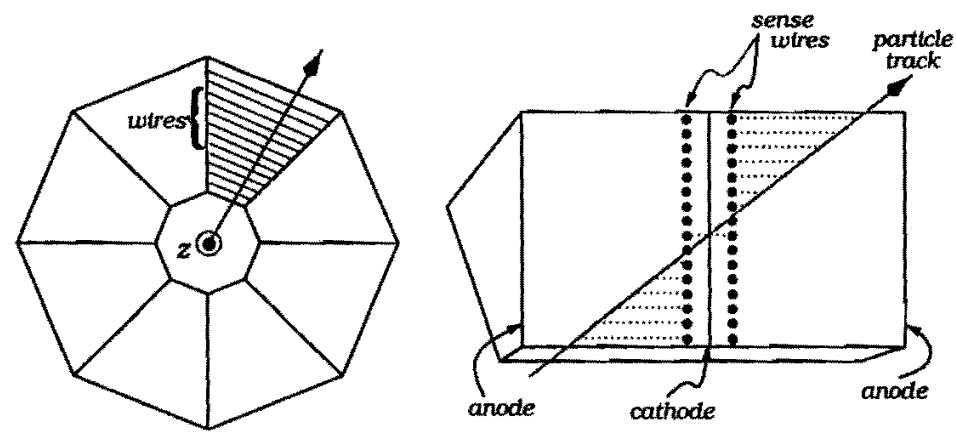

Figure 2.7: A schematic view of the VTX. 


\subsubsection{Central Tracking Chamber (CTC)}

The CTC covers the region $|z|<160 \mathrm{~cm}$ and $27.7<r<138 \mathrm{~cm}$, It contains a total of 6156 gold-plated tungsten sense wires ( $40 \mu \mathrm{m}$ diameter) arranged in 84 concentric layers. Figure 2.8 shows an $x-y$ view of the CTC end plate. These 84 layers are grouped together into 9 "superlayers". The superlayers are sub-divided into cells which contain series of sense wires, and field-shaping wires. Five of these superlayers are made of twelve layers that have their wires parallel to the $z$ axis (axial superlayers). The remaining four superlayers have their wires tilted at a $3^{\circ}$ angle with respect to the $z$ axis (stereo superlayers). The sense wires in each cell are aligned along $\mathrm{a} \sim 45^{\circ}$ angle with the radial direction This "Lorentz angle" is chosen so that the crossed electric and magnetic fields produce an azimuthal drift direction. It depends on the strength of the electric (1350 $\mathrm{V} / \mathrm{m})$ and magnetic $(1.4 \mathrm{~T})$ fields, and the choice of the gas $(49.6 \% \mathrm{Ar}, 49.6 \%$ ethane, and $0.8 \%$ alcohol). The maximum of the drift distance and drift time is $3.6 \mathrm{~cm}$ and 706 ns, respectively.

The signals from the sense wires are read with amplifiers and discriminators, then sent to time-to-digital-counters (TDC) which are able to record more than 7 hits per wire per event. The spatial resolution of each wire is $\sim 200 \mu \mathrm{m}$ in the azimuthal direction and that for the stereo wire is $\sim 200 \mu \mathrm{m} / \sin 3^{\circ}=4 \mathrm{~mm}$ in the $z$ direction. The hit efficiency is $\sim 98 \%$ per wire.

\subsubsection{Track Reconstruction}

Charged particles have a helical trajectory, and the helix is described by 5 "helix parameters", $h_{1, . .5}=\left(\rho, \phi_{0}, d_{0}, \cot \theta\right.$, and $\left.z_{0}\right)$. Figure 2.9 shows the definition of the track helix parameter. The first three parameters describe the circle in the $x-y$ plane. $\rho$ is radius of the circle in the $x-y$ plane and its sign is defined as positive for the positively charged particles. $\phi_{0}$ is the azimuthal angle of the track at its point of closest approach to the origin, and $d_{0}$ is the distance from the origin at that point. The momentum of the particle in the transverse plane $\left(p_{T}\right)$ is proportional to the $|\rho|$ and the magnetic field $B$, and described by the equation,

$$
p_{T}=0.2998 \cdot B \cdot|\rho|
$$

where $p_{T}$ is in $\mathrm{GeV} / c, \rho$ is in $\mathrm{m}$, and $B$ is in Testa.

$\cot \theta$ and $z_{0}$ determine the trajectory of the track in the $r z$ plane. $z_{0}$ is the $z$ coordinate at the point of closest to the $z$ axis, and $\theta$ is the polar angle of the track at 


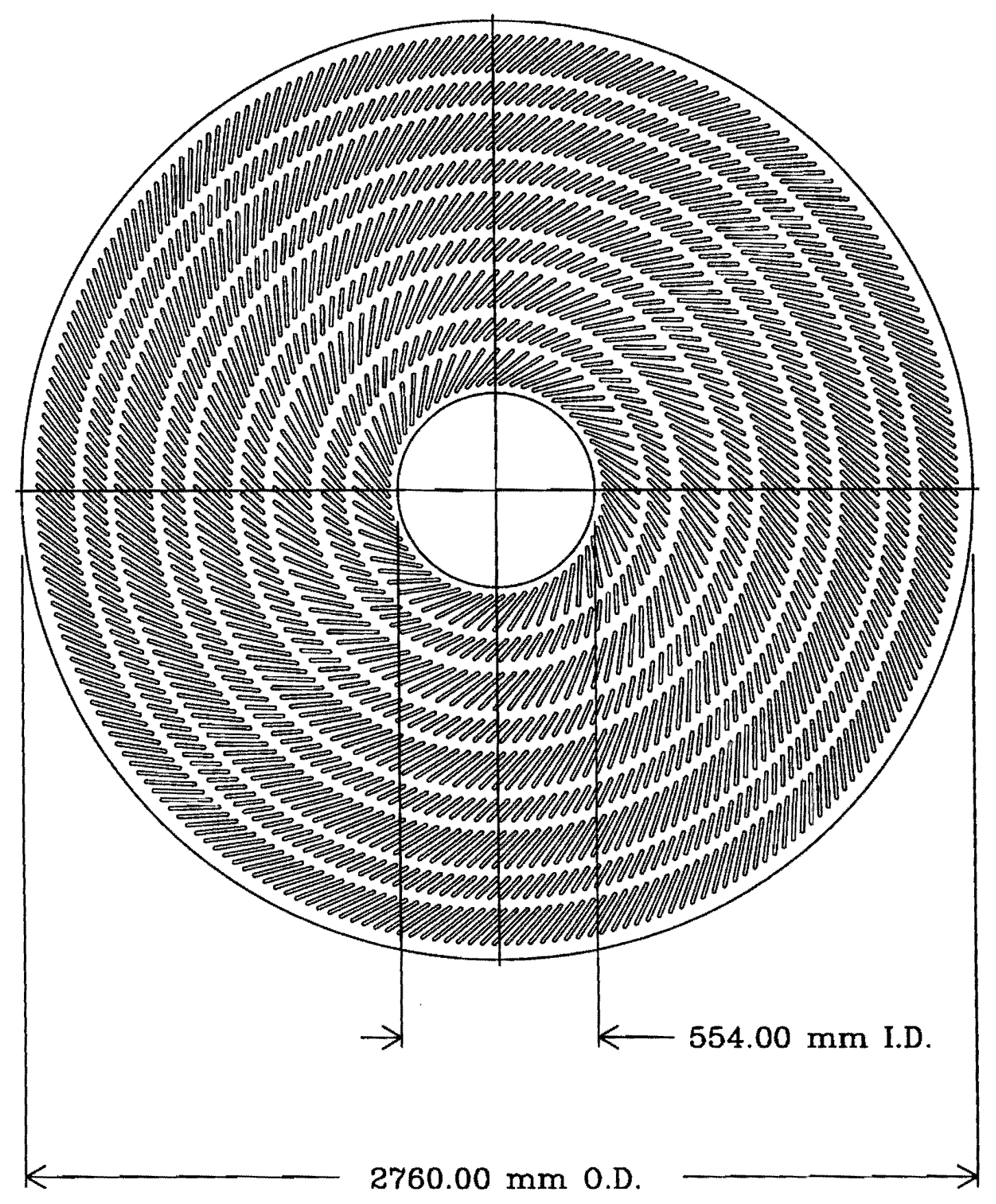

Figure 2.8: An $x-y$ view of the CTC end plate. 
that point.
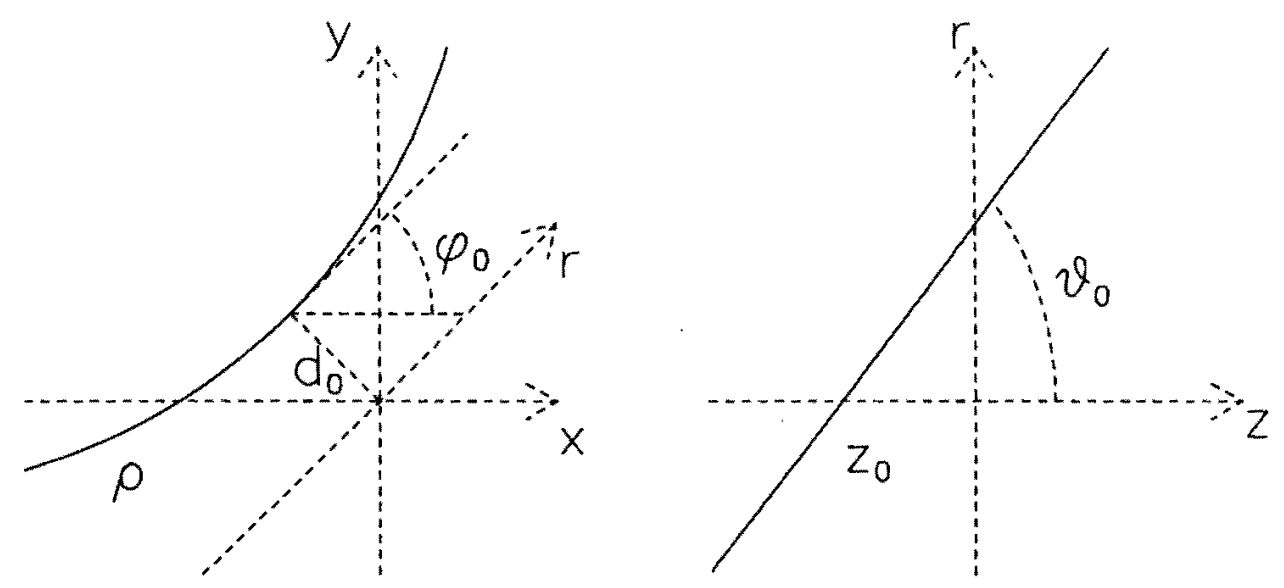

Figure 2.9: The definition of the track parameters.

The CTC provides three dimensional stand-alone tracking of charged particles (CTC track). The track reconstruction in the SVX is performed as follows, Starting from the CTC track, we include hits in the SVX which are found along the extrapolated track, if, for each SVX hit, the $\chi^{2}$ of the overall fit does not increase by more than 6 on average. The momentum resolution of a track reconstructed by using both the SVX and the CTC (simply called the SVX track) is $\sigma\left(p_{T}\right) / p_{T}=\left[\left(0.0009 p_{T}\right)^{2}+(0.0066)^{2}\right]^{1 / 2}$ with $p_{T}$ in units of $\mathrm{GeV} / c$, and the average impact parameter resolution for high $p_{T}$ tracks is 13 $\mu \mathrm{m}$.

\subsection{5 $d E / d x$}

Ionizing energy deposition of the particle in the material depends on the mass of the particle. This property gives useful information for the particle identification. Figure 2.10 shows the energy deposition of several charged particles in the $\mathrm{Ar}(80 \%)-\mathrm{CH}_{4}(20 \%)$ mixture [6]. We use number of the CTC wire hits $\left(N_{C T C}\right)$ which is related to the energy deposition of the particle in the CTC gas. It provides $0.7 \sigma(1.5 \sigma)$ of the $K-\pi(e-\pi)$ separation. The expected number of the CTC hits $N(P)$ and its uncertainty $\sigma N(P)$ depend on the particle $P$, and they are measured by using the CDF data for the electrons, muons, pions, kaons, and protons. The $d E / d x$ is defined for each particle $P$ as follows,

$$
d E / d x(P)=\frac{N_{C T C}-N(P)}{\sigma N(P)}
$$




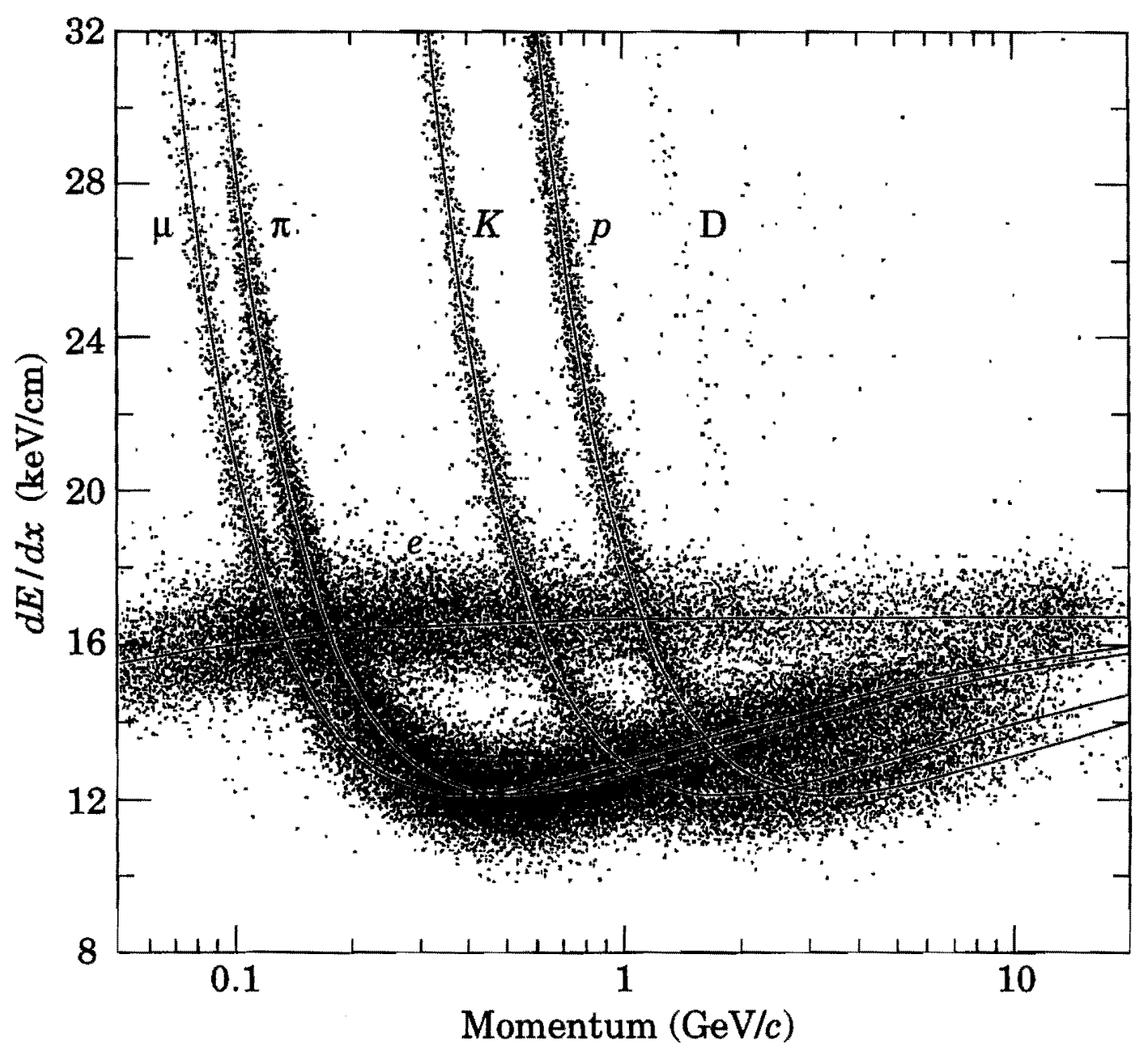

Figure 2.10: $d E / d x$ in the $8.5 \mathrm{~atm} A r-\mathrm{CH}_{4}$ as a function of the track momentum for several particles. 


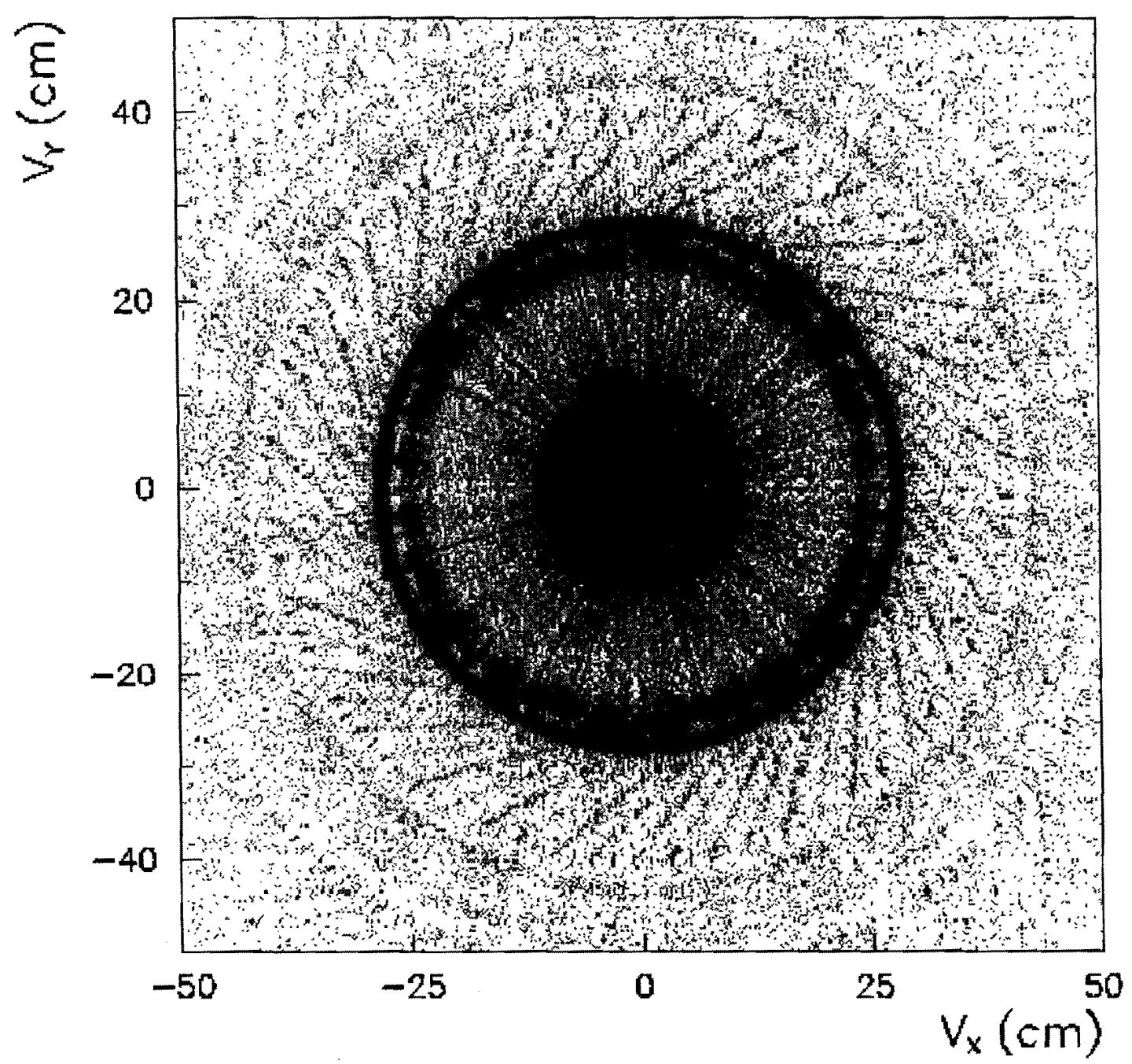

Figure 2.11: A $x y$-view cross section of the CDF inner detector by using the photon conversion vertex.

\subsubsection{Photon Radiator}

In this analysis, the photon from the radiative $B$ decay is identified by an electronpositron pair produced through the external photon conversion. The tracking detectors before the CTC, whose material thickness corresponds to $\sim 8 \% X_{0}$, where $X_{0}$ is the radiation length, are also used as radiators for the photon. Figures 2.11 and 2.12 show the conversion vertex position distribution in the $x y$-view and $r z$-view cross sections. In these plots, we can clearly see fine structures of the SVX, VTX, and CTC. Details of the conversion reconstruction methods are described in Sections 3.7 and 4.2. 


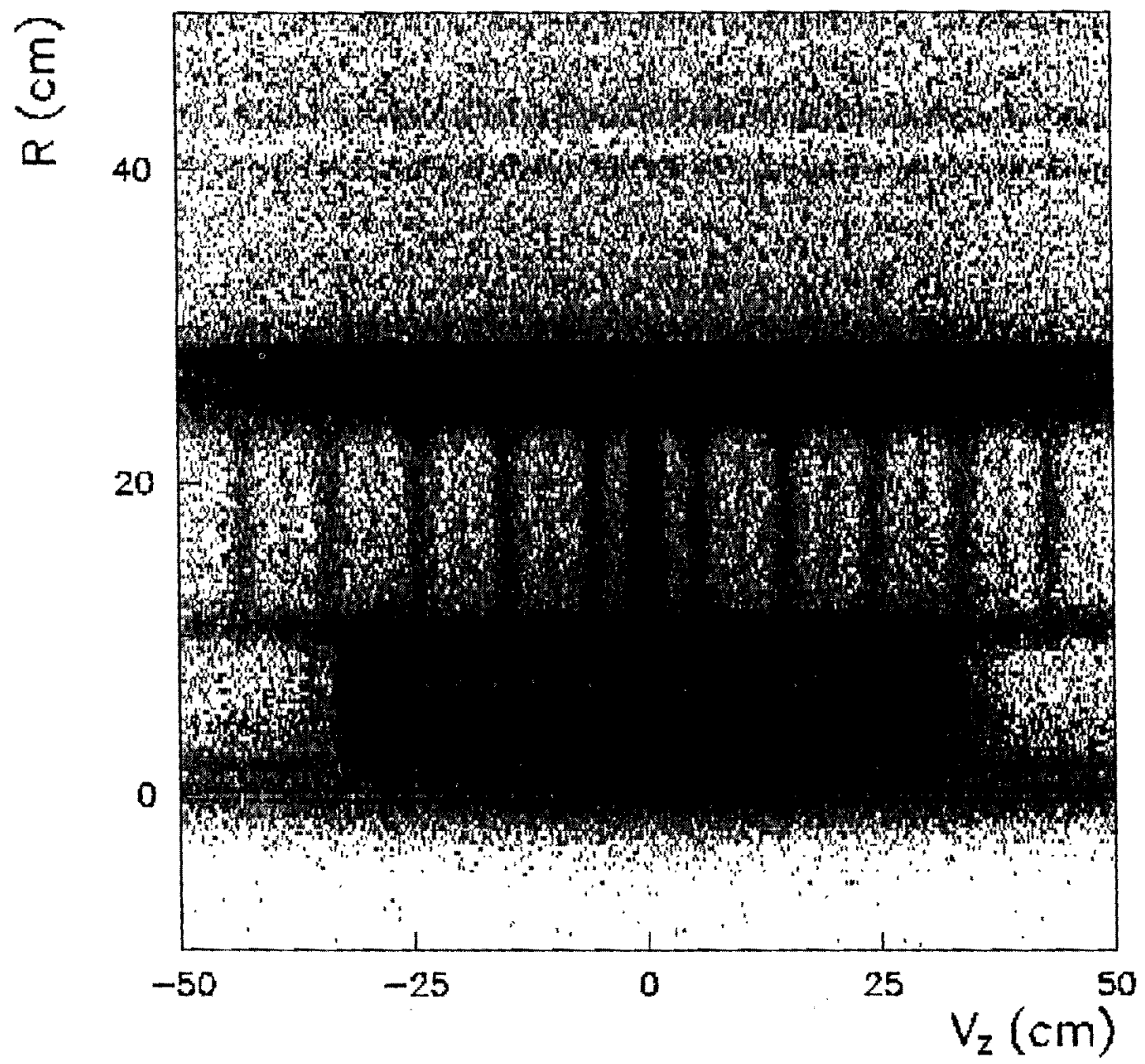

Figure 2.12: A rz-view cross section of the CDF inner detector by using the photon conversion vertex. 


\subsection{Calorimeters}

\subsubsection{Central Electromagnetic Calorimeter (CEM)}

The central calorimeters consist of two main components which can be viewed as hollow cylinders coaxial with the beamline. The inner component is the central electromagnetic calorimeter (CEM) and the outer one is the central hadronic calorimeter (CHA) [36]. The CEM [35] has an inner radius of $173 \mathrm{~cm}$ and a radial depth of $32 \mathrm{~cm}\left(\sim 18 X_{0}\right)$ and consists of 31 layers of $5 \mathrm{~mm}$ thick plastic scintillator interleaved with 30 layers of $1 / 8$ inch lead sheets. It provides full azimuthal coverage with its segmentation into 24 wedges, each covering $15^{\circ}$ in $\phi$. Each wedge is segmented in towers along the $z$ direction, each extending $\sim 0.11$ units in $\eta$ (Figure 2.13 ). The towers have a projective geometry pointing back to the nominal interaction point. The energy resolution of the CEM is $\sigma\left(E_{T}\right) / E_{T}=\left[(0.137)^{2} / E_{T}+(0.02)^{2}\right]^{1 / 2}$ with $E_{T}$ in units of $\mathrm{GeV}$.

\subsubsection{Central Strip Chamber (CES)}

Proportional strip chambers are inserted inside the CEM wedges at $r=184 \mathrm{~cm}$ and depth corresponding to the maximum average longitudinal development of electromagnetic showers $\left(5 X_{0}\right.$ from the CEM face or $6 X_{0}$ from the $p \bar{p}$ interaction point). The chambers have wires running along the $z$ direction and strips perpendicular to the wires. The task of these central strip chambers (CES) [35] is to determine the shower position and the shower transverse development as a means to distinguish electromagnetic showers induced by electrons and photons from hadrons. The shower position measurement has a resolution of $3 \mathrm{~mm}$ for $10 \mathrm{GeV}$ photons/electrons, reaching a plateau of $2 \mathrm{~mm}$ at $50 \mathrm{GeV}$.

A local coordinate system is defined for each CEM wedge. The $z_{C E S}$ axis is parallel to the global CDF $z$ axis, and the $x_{C E S}$ axis is parallel to the face of the CES, perpendicular to the $z_{C E S}$, and the origin of the $x_{C E S}$ corresponds to the half point of the wedge. Thus the wires and strips give the shower information of the $x_{C E S}$ and $z_{C E S}$ directions, respectively.

\subsubsection{Central Hadronic Calorimeter (CHA)}

The CHA has the same $\phi-\eta$ tower segmentation as the CEM. The CHA consists of 32 layers of $1 \mathrm{~cm}$ thick plastic scintillator interleaved with $2.5 \mathrm{~cm}$ steel sheets. In this 


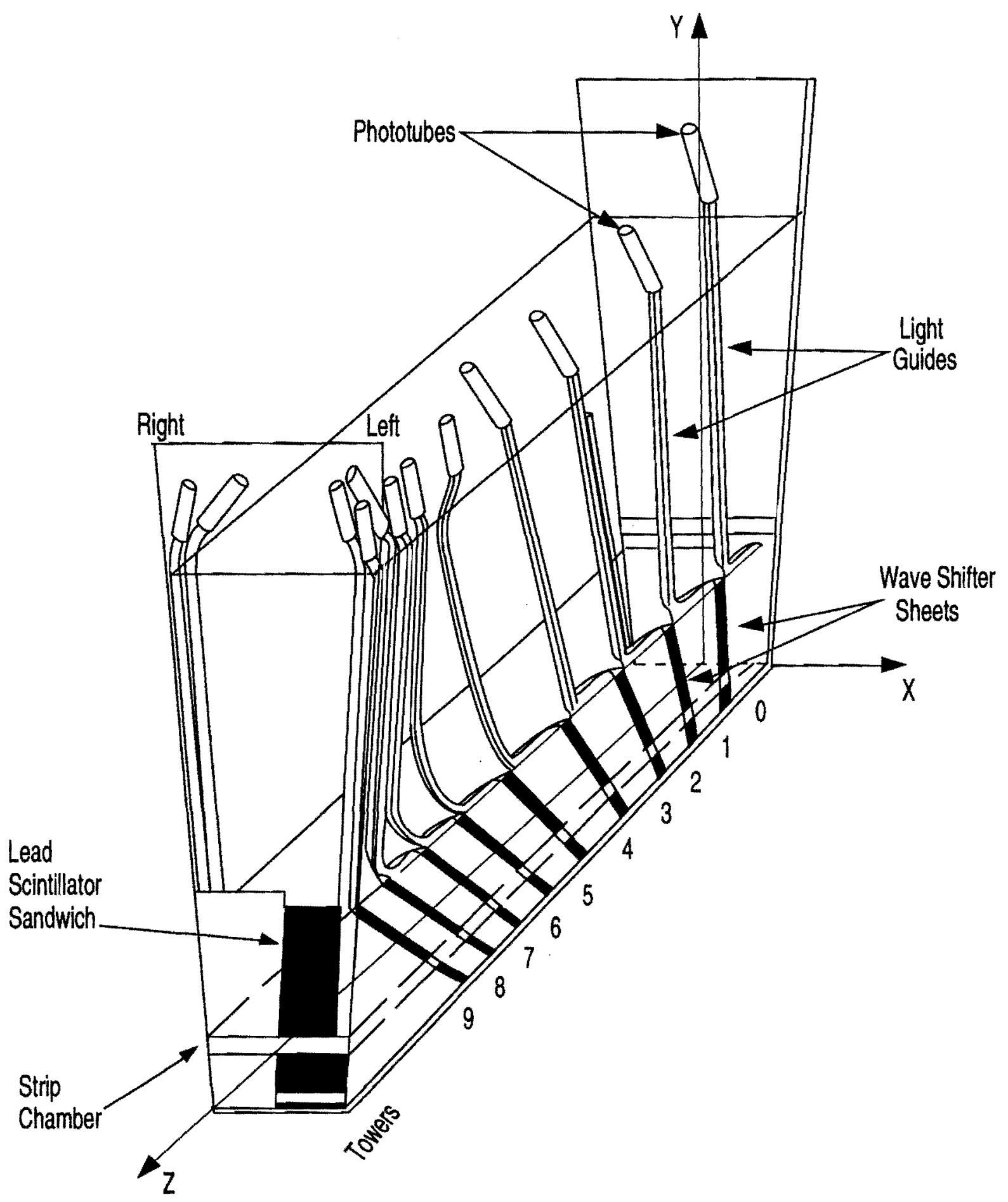

Figure 2.13: A isometric view of the central calorimeter. 
analysis, the CHA is only used to measure leakage of the electromagnetic showers to distinguish electrons and photons from hadrons.

\subsection{Trigger}

Table 2.1: Cross section and signal rate for various physics processes.

\begin{tabular}{l|ccc|}
\hline \hline Physics process & Cross section & Rate $^{1}$ & Events $/ 100 \mathrm{pb}^{-1}$ \\
\hline Bunch crossing & - & $286 \mathrm{kHz}$ & - \\
Minimum bias & $47 \mathrm{mb}$ & $470 \mathrm{kHz}^{2}$ & $4.7 \times 10^{12}$ \\
$b \bar{b}$ & $100 \mu \mathrm{b}$ & $1 \mathrm{kHz}$ & $1 \times 10^{10}$ \\
$b \bar{b} \rightarrow B_{d} X^{3}$ & $3 \mu \mathrm{b}$ & $30 \mathrm{~Hz}$ & $3 \times 10^{8}$ \\
$W \rightarrow e \nu$ & $2.5 \mathrm{nb}$ & $2.5 \times 10^{-2} \mathrm{~Hz}$ & $2.5 \times 10^{5}$ \\
$Z \rightarrow e e$ & $230 \mathrm{pb}$ & $2.3 \times 10^{-3} \mathrm{~Hz}$ & $2.3 \times 10^{4}$ \\
$t \bar{t}$ & $4.8 \mathrm{pb}$ & $4.8 \times 10^{-5} \mathrm{~Hz}$ & 480 \\
\hline \hline${ }^{1}$ at $\mathcal{L}=1.0 \times 10^{31} \mathrm{~cm}^{-2} \mathrm{~s}^{-1}$ & & \\
2 & & & \\
${ }^{3} p_{T}\left(B_{d}\right)>6 \mathrm{GeV} / c,\left|\eta\left(B_{d}\right)\right|<1.0$ &
\end{tabular}

The collision of the proton and antiproton bunches occurs every $3.5 \mu \mathrm{s}$, and the typical instantaneous luminosity is $1 \times 10^{31} \mathrm{~cm}^{-2} \mathrm{~s}^{-1}$ at the Tevatron. Table 2.1 shows the cross sections for various physics processes and their signal rates under the typical Tevatron environment. The event rate which we can record permanently is limited by the ability to write the data on tape $(\sim 10 \mathrm{~Hz})$ at the CDF. Note that even the $B_{d}$ meson production rate $(30 \mathrm{~Hz})$ exceeds the limit. 'Thus it is important to 'trigger' the event which we are interested in. Besides the recording rate, there are also several bottle necks for the CDF data taking system. Fort example the rate which we can reconstruct the events by using the same software code as the offline analysis is also limited ( $30 \mathrm{~Hz}$ ). Consequently, a three-level trigger system is employed at CDF [37]. The first two levels are a programmable hardware trigger and the third level is software and uses almost the similar quantities as the offline selection cut. Details of the trigger selection are described in Section 3.2 


\section{Chapter 3}

\section{Event Reconstruction}

In this chapter, we describe the selection criteria for the radiative $B$ signal events and the charmonium $B$ decays which are used for the reference sample. We also describe characteristics of the Monte Carlo sample which is used to evaluate the efficiency for the signal events. The final selection cut for the radiative $B$ decays is optimized to maximize the significance defined by $S / \sqrt{B}$, where the $S$ and $B$ are efficiencies for the signal and background, and we check whether the significant excess from the background is there.

\subsection{Event Selection Overview}

We reconstruct three channels of the exclusive radiative $B$ decays, namely, $\bar{B}_{d} \rightarrow \bar{K}^{* 0} \gamma$, $\bar{B}_{s} \rightarrow \phi \gamma$, and $\Lambda_{b} \rightarrow \Lambda \gamma$. The $\bar{B}_{d}, \bar{B}_{s}$, and $\Lambda_{b}$ hadrons consist of the $b$ qurak, and charge conjugate decays of the $B_{d}, B_{s}$, and $\bar{\Lambda}_{b}$ hadrons, which consist of the $\bar{b}$ qurak, are always implied throughout this paper unless mentioned otherwise. In this analysis, the photon is identified by an electron-positron pair produced through the external photon conversion before the CTC, and one of the conversion electrons with $E_{T}$ greater than $8 \mathrm{GeV}$ are served as a trigger for recording these events. We also identified the two charged tracks from the strange hadrons.

Figure 3.1 shows a schematic diagram of the $\bar{B}_{d} \rightarrow \bar{K}^{* 0}\left(\rightarrow K^{-} \pi^{+}\right) \gamma\left(\rightarrow e^{-} e^{+}\right)$ channel in the CDF detector. A $\bar{B}_{d}$ meson is produced at the primary vertex and decays into a photon and a $\bar{K}^{* 0}$ meson with a typical flight length of $2 \mathrm{~mm}$. The $\bar{K}^{* 0}$ meson decays to $K^{-} \pi^{+}$promptly, thus its decay vertex forms the $\bar{B}_{d}$ decay vertex. A $50 \%$ of the $K$ and $\pi$ from the $\bar{K}^{* 0}$ are reconstructed in the SVX, and gives a good information to separate the radiative $B$ signal and the background tracks from the primary vertex.

The signature of the $\bar{B}_{s} \rightarrow \phi \gamma$ is almost the same as that for $\bar{B}_{d} \rightarrow \bar{K}^{* 0} \gamma$. There is $\phi$ and $K^{+}$mesons instead of the $\bar{K}^{* 0}$ and $\pi^{+}$, respectively. Table 3.1 shows the mass and width of the bottom and strange hadrons. Since the $\phi$ meson has about 10 times narrower width $\left(4.4 \mathrm{MeV} / c^{2}\right)$ than that for the $\bar{K}^{* 0}\left(50.5 \mathrm{MeV} / c^{2}\right)$, we can easily obtain 
Table 3.1: Summary of the mass and width of the bottom and strange hadrons.

\begin{tabular}{|lcc|lcc|}
\hline \hline & Mass $\left(\mathrm{MeV} / c^{2}\right)$ & lifetime & & Mass $\left(\mathrm{MeV} / c^{2}\right)$ & Width,lifetime \\
\hline$B_{d}$ & 5279.2 & $468 \mu \mathrm{m}$ & $K^{* 0}$ & 896.1 & $50.5 \mathrm{MeV} / \mathrm{c}^{2}$ \\
$B_{s}$ & 5369.3 & $462 \mu \mathrm{m}$ & $\phi$ & 1019.4 & $4.43 \mathrm{MeV} / \mathrm{c}^{2}$ \\
$\Lambda_{b}$ & 5624. & $372 \mu \mathrm{m}$ & $\Lambda$ & 1115.7 & $7.89 \mathrm{~cm}$ \\
\hline \hline
\end{tabular}

better reduction for the combinatorial background by applying a tighter mass window cut.

Figure 3.2 shows schematic diagram of the $\Lambda_{b} \rightarrow \Lambda \gamma$ channel. One of the differences of the $\Lambda_{b}$ channel from the $\bar{B}_{d}$ and $\bar{B}_{s}$ channels is caused by a longer lifetime of $\Lambda$ (cr $\sim 8$ $\mathrm{cm})$. Thus knowledge of the $\Lambda$ decay vertex alone is not enough to decide whether the $\Lambda$ comes from a displaced vertex or the primary vertex. However the impact parameter of the $\Lambda$ around the primary vertex is still useful. We found a $10 \%$ of the $\Lambda$ from $\Lambda_{b}$ is reconstructed in the SVX, and the resolution of the impact parameter is fine enough for reject the $\Lambda$ from the primary vertex. On the other hand we can easily improve the $S / B$ of the $\Lambda$ by using the reconstructed $\Lambda$ decay vertex to be separated from the primary vertex, and the energy deposition of the proton in the CTC $(d E / d x)$ to be consistent with the expectation as a proton.

We also reconstruct tqo of the exclusive charmonium $B$ decay channels, namely, $\bar{B}_{u} \rightarrow J / \psi K^{-}$and $\bar{B}_{d} \rightarrow J / \psi \bar{K}^{* 0}$. In this analysis the $\bar{B}_{u} \rightarrow J / \psi K^{-}$decay, which has a similar signature as the radiative $B$ decays and sufficient statistics $(\sim 50$ events $)$, is used for normalizing the efficiency for the radiative $B$ decays.

\subsection{Trigger}

In this analysis, we use a central electron trigger with an $E_{T}$ threshold of $8 \mathrm{GeV}$.

\subsubsection{Level 1}

The Level 1 trigger decision is made every beam crossing and reduce the trigger rate from $286 \mathrm{kHz}$ to $\sim 1 \mathrm{kHz}$. It is based on the calorimeter information. The central calorimeter information is summed, separately for the electromagnetic and hadronic parts, into logical "trigger towers", each encompassing enough physical towers to extend to 0.2 units in $\eta$ and $15^{\circ}$ in $\phi$. The Level 1 trigger accepts an event if there is any trigger tower with transverse energy above the preset programmable threshold. The $E_{T}$ 


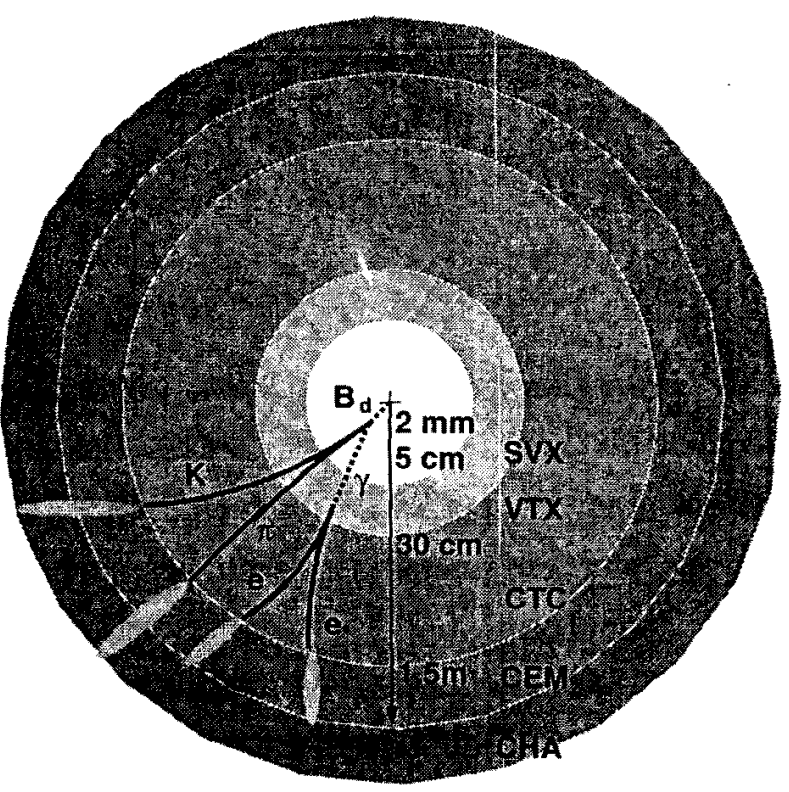

Figure 3.1: Schematic diagram of the $\bar{B}_{d} \rightarrow \bar{K}^{* 0} \gamma$ reconstruction.

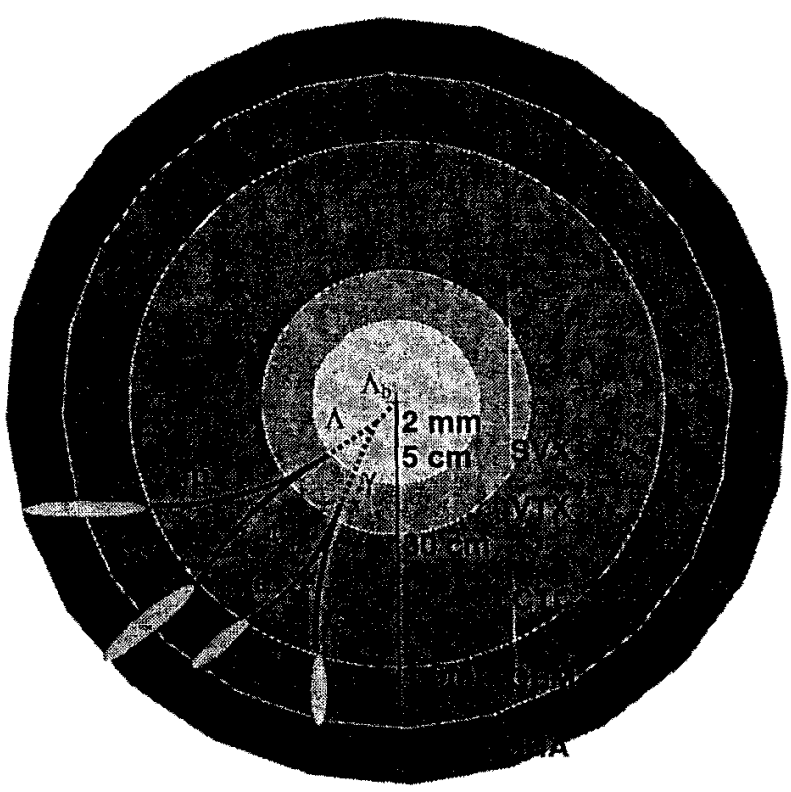

Figure 3.2: Schematic diagram of the $\Lambda_{b} \rightarrow \Lambda \gamma$ reconstruction. 
is calculated by using the central position of the trigger tower and assuming the primary vertex to be $z=0$. In this analysis, we use a trigger which requires a trigger tower with electromagnetic energy $E_{T}>8 \mathrm{GeV}$.

\subsubsection{Level 2}

The Level 2 trigger decision is made only for the events passing the Level 1 trigger, and based on the combination of the calorimeter and tracking detector information. Level 2 organizes the list of calorimeter trigger towers into clusters. The CEM trigger towers serve as seeds for the clusters and adjacent trigger towers are added to the cluster if they had sufficient energy (typically $1 \mathrm{GeV}$ less than the seed tower threshold). The raw pulse height of the CES wire is summed over neighboring 4 strips and the Level 2 trigger accepts a cluster with its pulse height above the preset programmable threshold. A hardware track processor (CFT) [38] searches for tracks in the CTC using hits in the axial CTC layers, thus reconstructing two dimensional $(r-\phi)$ tracks. It classifies the tracks into lists of eight $p_{T}$ bins, one for each sign of curvature, covering the entire $p_{T}>2$ $\mathrm{GeV} / c$ range. The momentum resolution of the CFT is $\sigma\left(1 / p_{T}\right)=0.03(\mathrm{GeV} / c)^{-1}$, and Figure 3.3 shows the efficiency curves for the track to be fined as a CFT track bin 0-5 as a function of the CTC $p_{T}$ reconstructed offline.

The parts of Level 2 which look for electrons require that CEM and CES clusters has a CFT track pointing to the cluster. In this analysis, we use a trigger which require both CEM and CES clusters with $E_{T}>8 \mathrm{GeV}$ to be pointed by a bin 4 CFT track $\left(90 \%\right.$ efficiency for $\left.p_{T}=7.5 \mathrm{GeV} / c\right)$.

\subsubsection{Level 3}

The Level 3 trigger accepts electromagnetic clusters as electron candidates if their energy profiles of the longitudinal and the transverse development of the shower are consistent with expectation as an electron. For electrons, a three dimensional track reconstructed in CTC above some $p_{T}$ threshold is required to point to the electromagnetic cluster. At the Level 3, the calorimeter trigger towers are replaced by the actual physical tower segmentation $\left(\Delta \eta, \Delta \phi=0.11,15^{\circ}\right)$, and the software reconstructed CTC track with a crude calibration constant is available. An electromagnetic cluster in Level 3 is defined in the same way as the offline, and details of these requirements are discussed in Section 3.4 


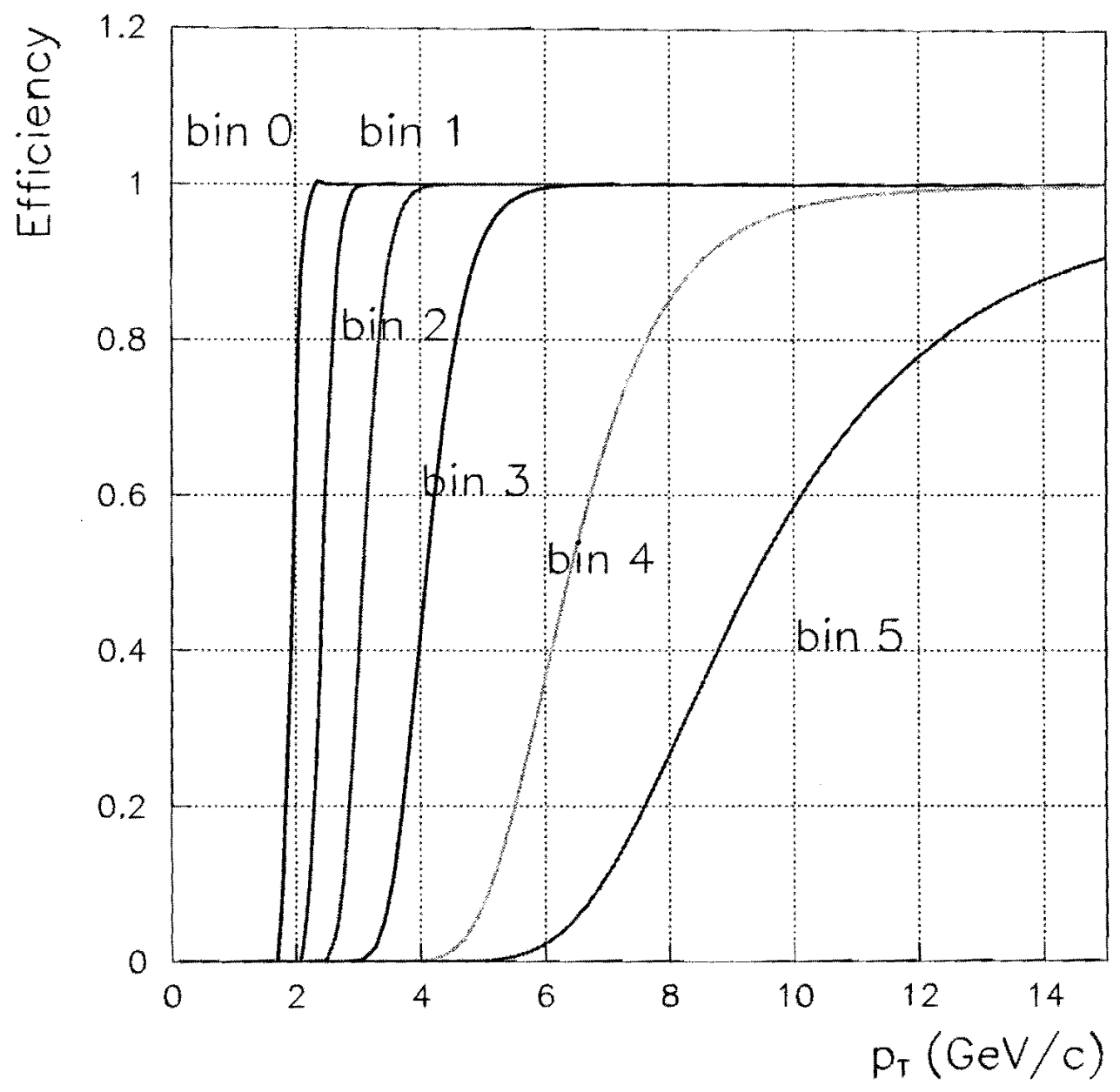

Figure 3.3: CFT bin 0-5 efficiency curves as a function of the $p_{T}$. 


\subsection{Monte Carlo Sample}

We use the Monte Carlo sample for estimating the selection cut efficiency. The sample simulates the $B$ hadron production and the CDF detector response. The data format of the MC sample is the same as real data and we can apply the same selection cuts for the MC sample as real data.

\subsubsection{B Hadron Generator}

We use a toy Monte Carlo program to simulate the $B$-hadron production and decay. This generates $b$ quarks with a given $p_{T}$ and $|\eta|$ distribution based on a next-to-leading order calculation [28] with the renormalization scale $\mu=\mu_{0} \equiv \sqrt{p_{T}^{2}+m_{b}^{2}}$ (where the $b$-quark mass $m_{b}$ is set to $4.75 \mathrm{GeV} / c^{2}$ ) and the MRSD0 proton parton distribution function [29]. We generate $b$-quarks with $p_{T}>6 \mathrm{GeV} / c$ and $|\eta|<1.5$. The fragmentation model by Peterson et al. [30] is used with a value of the fragmentation parameter $\epsilon_{b}=0.006$. The CLEO MC is then used to describe the $B$-hadron decays [39]. The lifetimes used in the simulation are the world average values [6]. Note that the Monte Carlo only generates radiative $B$ decay signals and there are no particles from the other $b$-quark or fragmentation, etc.

\subsubsection{Detector Simulation}

We use the fast detector simulation (QFL) which is based on the parameterization of the several detector resolution, EM and hadronic shower profile, etc, tuned by using the real data. Here we only list the basic feature of the detector simulation.

\section{- Primary vertex}

The primary vertex of the CDF is assumed to be Gaussian distribution with $\left(\sigma_{x}\right.$, $\left.\sigma_{y}, \sigma_{z}\right)=(25 \mu \mathrm{m}, 25 \mu \mathrm{m}, 30 \mathrm{~cm})$. The QFL displaces the primary vertex according to the distribution.

- CTC track simulation

The QFL does not simulate the CTC track from the wire hit level, however the number of the wire hits for each superlayer is simulated according to the wire hit efficiency measured in the data. The helix parameter of the track is simulated by smearing the generator level information according to the error matrix measured in the data. 
- SVX track simulation

The QFL has the geometry database of each silicon strip position, and simulates the SVX layer hits. The reconstruction of the SVX track is done by the same procedure as the real data, i.e., extrapolating the CTC track to the SVX layer position, finding the strip cluster matching the CTC track, and fitting by combining the helix parameter of the CTC track and silicon strip position.

- Calorimetry

The CEM, CES and CHA, response simulation for the electrons, photons, and hadrons based on the test beam data.

- Photon conversion, bremsstrahlung, multiple scattering

The QFL has a rough geometry database of the detector material, for example, the SVX is simplified as 4 cylinders, corresponding to the layer 1,2,3, and 4 . The photon conversion, bremsstrahlung, and multiple scattering are simulated based on the database.

\subsection{Electron Reconstruction}

The Level 3 and offline electron quantities are measured with the CEM, CHA, CES, and CTC. Table 3.4.7 shows a list of the selection cuts. Approximately 3 million electron candidates survived these criteria. Composition of the electron trigger sample is, the semileptonic $B$ decays (30\%), the semileptonic $c$ decays (20\%), the photon conversions $(30 \%)$, and the misidentified hadrons $(20 \%)$. In this section we describe the details of the electron quality cut parameters.

\subsubsection{Electromagnetic Energy: $E_{T}$}

At the Level 3 and offline reconstruction, the trigger towers are replaced by the actual physical tower segmentation $\left(\Delta \eta, \Delta \phi=0.1,15^{\circ}\right)$. The $E_{T}$ is calculated with the EM energy deposition in the calorimeter cluster $(E)$ and the $\theta$ of the CTC track by $E_{T}=$ $E \sin \theta$.

\subsection{2 $E / p$}

The $E / p$ is defined as a ratio between the CEM cluster energy and the CTC momentum. The $E / p$ is ideally 1.0 within the detector resolution of the $E_{T}$ and $p_{T}$. However, there 
are two additional effects to change the $E / p$ distribution.

- Bremsstrahlung

Electrons lose their energy by photon bremsstrahlung in the detector material and solenoidal magnetic field. Since the bremsstrahlung photon is emitted to the same direction of the electron, the photon is mostly in the same CEM cluster of the electron and the CEM measures the orininal electron energy. On the other hand the CTC measures the momentum of the track after the energy loss in the material before the CTC. This causes $\sim+6 \%$ of the systematic shift in the averaged $E / p$ distribution.

- Underlying Events

The CEM energy is piled up by the underlying events which include particles from the $B$ decay themselves, the fragmentation, and the multiple collision. The effect strongly depends on the physics process and the instantaneous luminosity. If the electron is originating from the photon conversion, we expect another electron to be around the trigger electron, and it can increase the CEM energy. If the electron is originating from the process such as the $\pi^{0} \rightarrow \gamma \gamma \rightarrow e^{+} e^{-} \gamma$ decay, we expect the other photon to be around the electron, and it increases the CEM energy.

We do not cut on the $E / p$ by these reasons.

\subsection{3 $\mathrm{Had} / \mathrm{EM}$}

The $\mathrm{Had} / E M$ is a ratio of the energy deposition in the CHA and the CEM. Each of the energy is summed over the seed tower and two neighboring towers. Since the CEM has an $18 X_{0}$ of the transverse depth, the expected energy leakage of electron to the CHA is less than one percent, and the hadronic energy is usually dominated by the underlying events. Thus, the hadronic energy cut does not only work as the electron identification but also requires the electron to be isolated from the other products.

If there are more than one tracks to point the calorimeter cluster, we expect the $H a d / E M$ to become significantly higher than that for a single electron. Thus the $\mathrm{Had} / \mathrm{EM}$ requirement which we required offline is the $N_{t r k}$ dependent. If the $N_{t r k}=1$ $(>1)$ we require $\mathrm{Had} / \mathrm{EM}<0.04(0.1)$. 


\subsubsection{L}

The $L_{\text {share }}$ represents the consistency of the electron shower profile in the $\eta$ direction with the test beam data. Ths $L_{\text {share }}$ is defined by

$$
L_{\text {share }}=0.14 \sum_{k=1}^{3} \frac{M_{k}-P_{k}}{\sqrt{0.14^{2} E_{T}+\left(\Delta P_{k}\right)^{2}}},
$$

where the $M_{k}\left(P_{k}\right)$ is the measured (expected) transverse energy in the CEM tower $k, \Delta p_{K}$ is the uncertainty for the $P_{k}$, and $k$ is summed over the seed tower and two neighboring towers.

The $L_{\text {share }}$ is also an underlying event dependent quantity. We require $L_{\text {share }}<$ 0.002 at both the Level 3 and offline selection.

\subsection{5 $\chi_{\text {strip,wire }}^{2}$}

The $\chi_{s t r i p}^{2}$ and $\chi_{\text {wire }}^{2}$ describe the consistency of the measured shower profiles and those determined using test beam data in $x_{C E S}$ and $z_{C E S}$ directions, respectively. Since the CES

\subsection{6 $\Delta z, \Delta x$}

The CES gives the position of the center of the electromagnetic shower, and their expectations are obtained by extrapolating the CTC track to the radius of the CES. The $\Delta z$ and $\Delta x$ represent the difference between the two positions in the direction of the $z_{C E S}$ and $x_{C E S}$ axis, respectively.

\subsubsection{Comparison between the Data and MC}

Figures 3.4 and 3.5 show the distributions of the electron quality cut parameters. These are obtained from the partner electron of the $J / \psi \rightarrow e e$ candidates (Section 3.6). Distributions for the data (points) and MC (histograms) are shown. The MC reproduces the shower maximum quantities (Figure 3.5) very well. However there are significant difference between the data and $\mathrm{MC}$ for the calorimeter (Figure 3.4) quantities which are caused by the underlying energy. This difference can be a source of the systematic uncertainty of this analysis. 
Table 3.2: Electron quality cut in Level 3 and offline selections.

\begin{tabular}{|l|l|l|}
\hline \hline Parameter & Level 3 & Offline \\
\hline$p_{T}$ & $6.0 \mathrm{GeV} / c$ & $6.0 \mathrm{GeV} / c$ \\
\hline$E_{T}$ & $7.5 \mathrm{GeV}$ & $8.0 \mathrm{GeV}$ \\
\hline$E / p$ & N/A & N/A \\
\hline Had $/ E M$ & 0.125 & $0.04\left(N_{3 D}=1\right)$ \\
& & $0.1\left(N_{3 D} \geq 1\right)$ \\
\hline$L_{\text {share }}$ & 0.2 & 0.2 \\
\hline$\chi_{\text {strip }}^{2}$ & 10.0 & 10.0 \\
\hline$\chi_{\text {wire }}^{2}$ & 10.0 & 10.0 \\
\hline$\Delta z$ & $10.0 \mathrm{~cm}$ & $3.0 \mathrm{~cm}$ \\
\hline$\Delta x$ & $3.0 \mathrm{~cm}$ & $1.5 \mathrm{~cm}$ \\
\hline
\end{tabular}
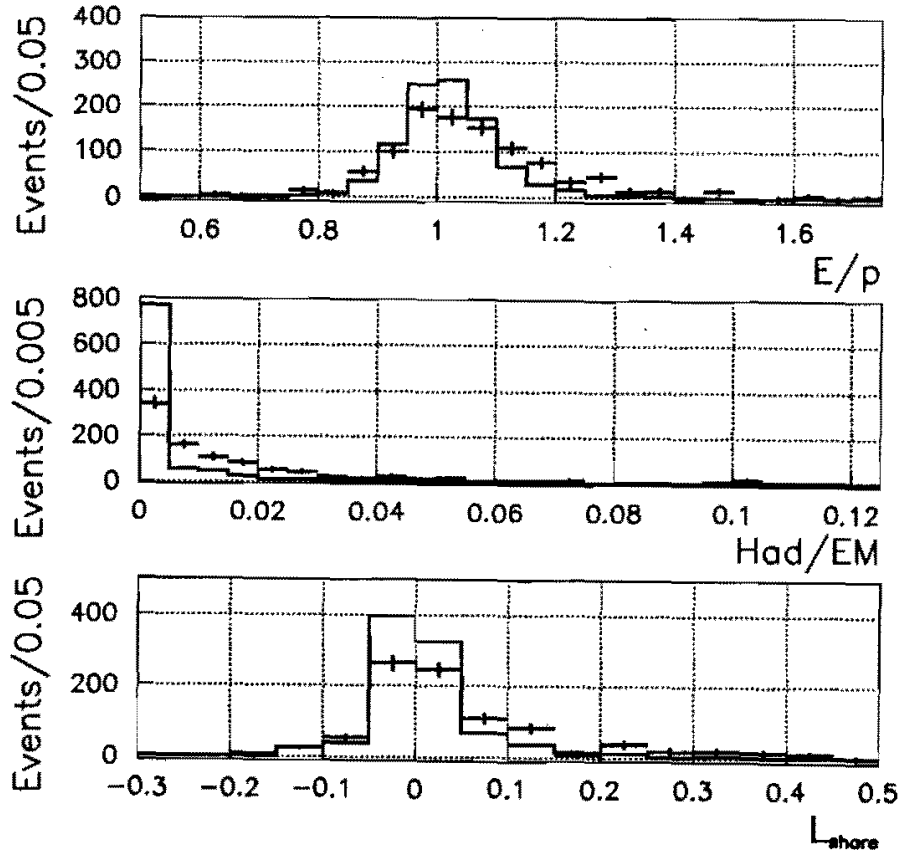

Figure 3.4: Distributions of the electron quality cut parameters, $E / p$, $\mathrm{Had} / \mathrm{EM}$, and $L_{s h a r e}$, for the partner electron of the $J / \psi \rightarrow e e$ candidates (points) and the $J / \psi K \mathrm{MC}$ sample (histograms). 

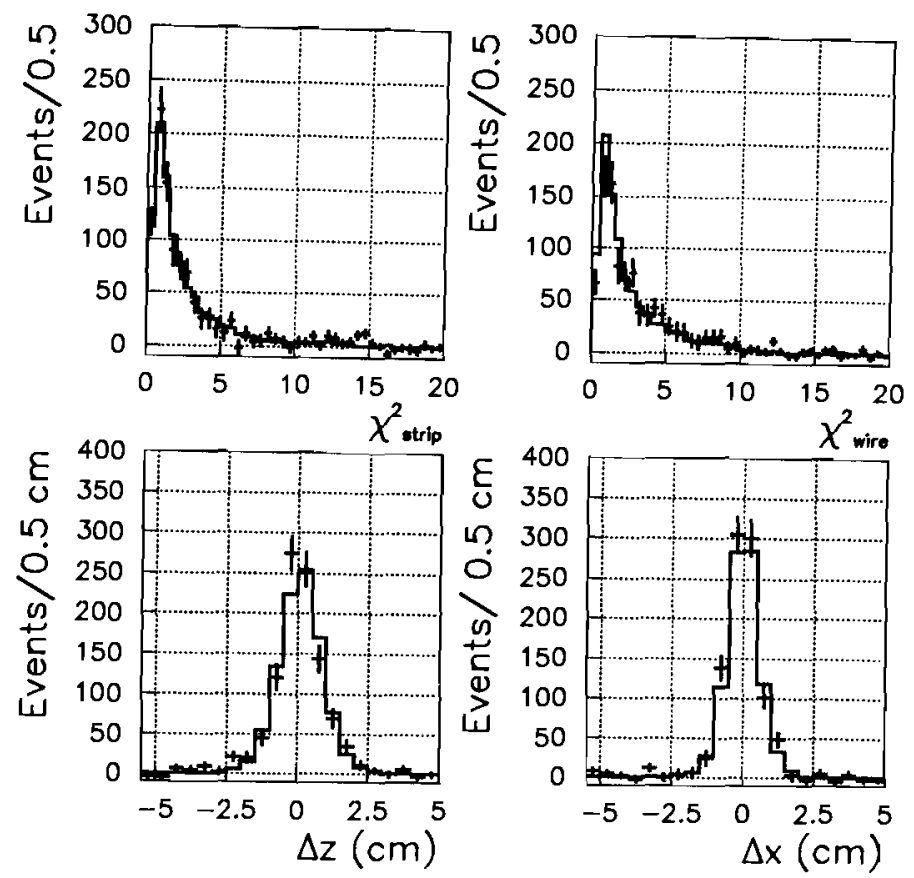

Figure 3.5: Distributions of the electron quality cut parameters, $\chi_{\text {strip,wire }}^{2}$ $\Delta z, \Delta x$, for the partner electron of the $J / \psi \rightarrow e e$ candidates (points) and the $J / \psi K$ MC sample (histograms).

\subsection{Vertex Fitting}

The $B$-hadron reconstruction in this analysis is based on the charged tracks, and there are several constraints for these track combinations caused by the decay chain.

- 2 or more tracks are associate with the same vertex (common vertex constraint) This is applied for the decay vertex such as the $J / \psi \rightarrow e e$ and $K^{* 0} \rightarrow K \pi$.

- In the photon conversion, the two electrons are coming from the same vertex, and also the two tracks are parallel at the vertex (conversion constraint).

- 2 or more track system points to a vertex (pointing constraint). This is applied for the combination such as $K^{* 0} \gamma$ points to the primary vertex and $\gamma$ points to the $K^{* 0}$ decay vertex.

Under these constraints, we fit the track helix parameters to minimize the $\chi^{2}$. For the vertex constraint fit, the $\chi^{2}$ is given by

$$
\chi^{2}=\sum \xi_{i}^{t} G_{i}^{-1} \xi_{i}
$$


where $G$ is the error matrix for the track helix parameter and $\xi$ is the parameter difference between the fitted $\left(\rho^{\prime}, \phi_{0}^{\prime}, \ldots\right)$ and input $\left(\rho, \phi_{0}, \ldots\right)$ value

$$
\xi=\left(\begin{array}{c}
\Delta \rho \\
\Delta \phi_{0} \\
\Delta d_{0} \\
\Delta \cot \theta \\
\Delta z_{0}
\end{array}\right)=\left(\begin{array}{c}
\rho^{\prime}-\rho \\
\phi^{\prime}-\phi_{0} \\
d_{0}^{\prime}-d_{0} \\
\cot \theta^{\prime}-\cot \theta \\
z_{0}^{\prime}-z_{0}
\end{array}\right) .
$$

There are two merits for using the fitting technique: 1) resolutions for the fitted parameters are improved; 2) the $\chi^{2}$ cut is useful for the background reduction.

3.6 $J / \psi \rightarrow e^{+} e^{-}$

A $J / \psi \rightarrow e^{+} e^{-}$candidate is formed by the electron candidate and an oppositely charged track with $p_{T}>1.0 \mathrm{GeV} / c$. A fit with the common vertex constraint is made by using the CTC tracks. The CL of the fit is required to be greater than $0.1 \%$.

To improve the signal-to-background ratio $(S / B)$ of the $J / \psi \rightarrow e e$ candidates, we require quality cuts for the the partner electron. The low $p_{T}(\sim 2 \mathrm{GeV} / c)$ electron is different from the high $p_{T}(>8 \mathrm{GeV} / c)$ electron. For example, $d E / d x$ in the CTC is very useful for the soft electron identification. The $d E / d x$ is calculated with an electron assumption (Equation 2.2). Thus the parameter is the Gaussian distribution with average $=0$ and sigma $=1$ for the real electrons. We require $d E / d x>-1.27$ for the partner electrons, which corresponds a $90 \%$ efficiency. We also require the partner electrons to satisfy $E / p>0.5$, where the CEM energy is not clustered energy but the energy in the CEM tower which is associated the CTC track.

Figure 3.6 shows the dielectron invariant mass distribution, and we found $\sim 8000$ of $J / \psi \rightarrow e e$ candidates above the background. The $S / B$ of the $J / \psi \rightarrow e e$ candidates is about $1 / 2$. The background is dominated by the combinatorial background of the trigger electron and a hadron track misidentified as an electron. There are another backgrounds coming from the sequential $b \rightarrow c \ell \nu \rightarrow s \ell \ell \nu \nu$ decay events.

There are two relevant effects by the bremsstrahlung for the $J / \psi \rightarrow e e$ reconstruction,

- The mass distribution in the $J / \psi$ peak region is not ideal Gaussian, but has a tail to the lower mass region. It is caused by the energy loses due to the bremsstrahlung. Thus we need to treat the $J / \psi$ mass window cut efficiency carefully. 
Table 3.3: Event selection criteria.

\begin{tabular}{|l|l|}
\hline Track (CTC) & 3D track \\
& $\geq 2$ axial CTC superlayers with $\geq 4$ hits \\
& $\geq 2$ stereo CTC superlayers with $\geq 2$ hits \\
& Covariance matrix scale factor 2.4 \\
& CDF material scale factor 1.31 \\
Track (SVX) & Retrack with the best alignment data \\
& Number of good hits $\geq 3$ \\
& $\chi^{2} /$ dof $<6$ \\
& Good CTC track \\
\hline$J / \psi \rightarrow e e$ & Electron + oppositely charged CTC track \\
& $p_{T}>1.0 \mathrm{GeV} / c$ \\
& $d E / d x \geq-1.27$ \\
& $E / p \geq 0.5$ \\
& Common vertex fit \\
& prob $\left(\chi^{2}\right)>0.1 \%$ \\
\hline$\gamma \rightarrow e e$ & Electron + oppositely charged CTC track \\
& $p_{T}>0.5$ GeV $/ c$ \\
& Conversion constraint fit \\
& prob $\left(\chi^{2}\right)>0.1 \%$ \\
& $R<30 \mathrm{~cm}$ \\
\hline
\end{tabular}

- Since the error matrix of the CTC track takes no account of the bremsstrahlung, the reconstructed position of the $J / \psi$ decay vertex and $\chi^{2}$ of the fitting are biased (Figure 3.7). Thus we need to treat the fitting CL cut efficiency carefully.

\subsection{Photons}

A $\gamma$ conversion candidate is formed by the trigger electron candidate and an oppositely charged track with $p_{T}>0.5 \mathrm{GeV} / c$. A conversion constraint fit is made with two CTC track information and the confidence level (CL) of the fit is required to be greater than $0.1 \%$. We obtained $\sim 850 \mathrm{k}$ of $\gamma$ conversion candidates. Figure 3.8 shows the radius distribution of the conversion vertex. The candidates with the conversion points inside the beampipe $(2.5 \mathrm{~cm})$ are dominated by real electron-positron pairs from Dalitz $\pi^{0}$ and $\eta$ decays. We also see several peaks corresponding to each CDF detector component, 


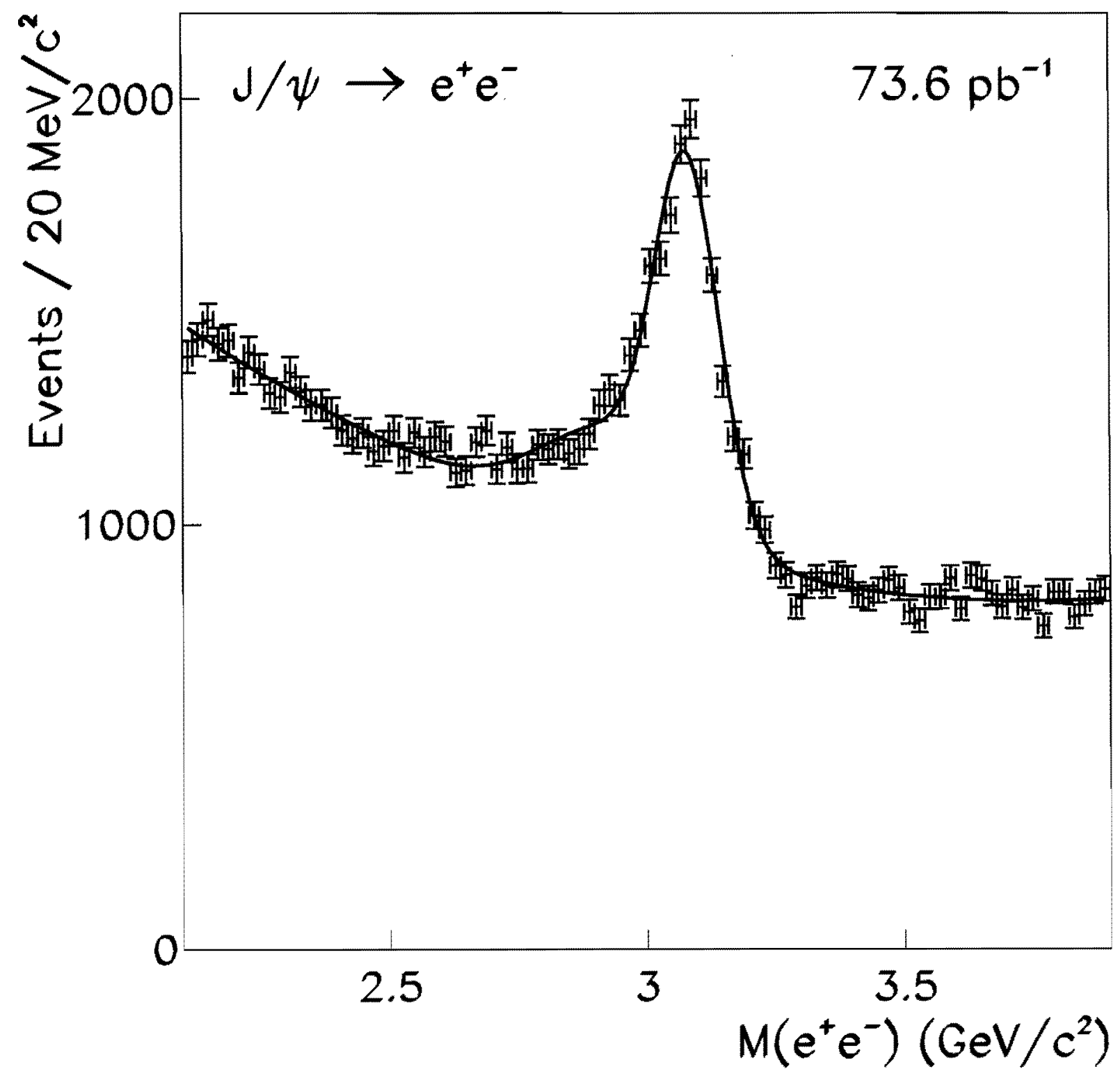

Figure 3.6: Trigger $J / \psi \rightarrow$ ee candidates in the data. 

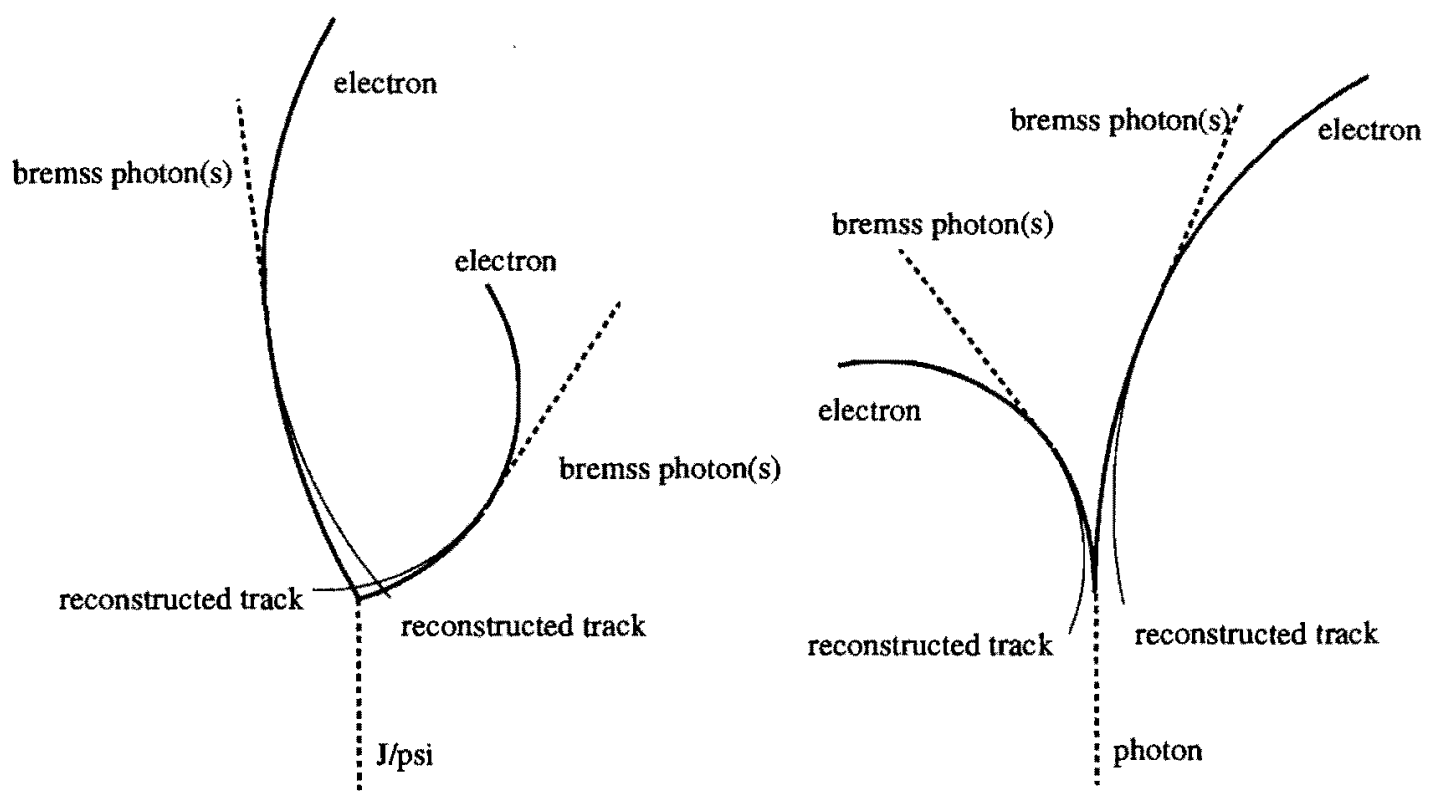

Figure 3.7: Schematic view of the effect of bremsstrahlung to the conversion reconstruction.

the SVX Layer 1, 2, 3, 4, VTX inner wall, VTX outer wall, and CTC can. The $x y$ and $r z$ views of the vertex distributions which are already shown in Figures 2.11 and 2.12 provide more detailed structure of the CDF inner detector. These distributions are used for calibrating the photon conversion probability.

There are similar problems caused by the bremsstrahlung for the conversion reconstruction,

- Since the conversion electron produced at outer radius pass through less materials, the effect of the bremsstrahlung is smaller than that for the $J / \psi \rightarrow e e$.

- Since the constraints for the conversion reconstruction are tighter than that for the $J / \psi \rightarrow e e$, the effect for the bremsstrahlung can be larger.

\subsection{Radiative $B$ Signal Reconstruction}

\subsection{1 $\quad \bar{B}_{d} \rightarrow \bar{K}^{* 0} \gamma$ and $\bar{B}_{s} \rightarrow \phi \gamma$}

For each $\gamma$ conversion candidate in an event we search for $\bar{B}_{d} \rightarrow \bar{K}^{* 0} \gamma$ and $\bar{B}_{s} \rightarrow \phi \gamma$. We first require the distance of the conversion vertex from the nominal beam line ( $z$-axis in the CDF coordinate) to be less than $30 \mathrm{~cm}$ in order to ensure that it is in the well 


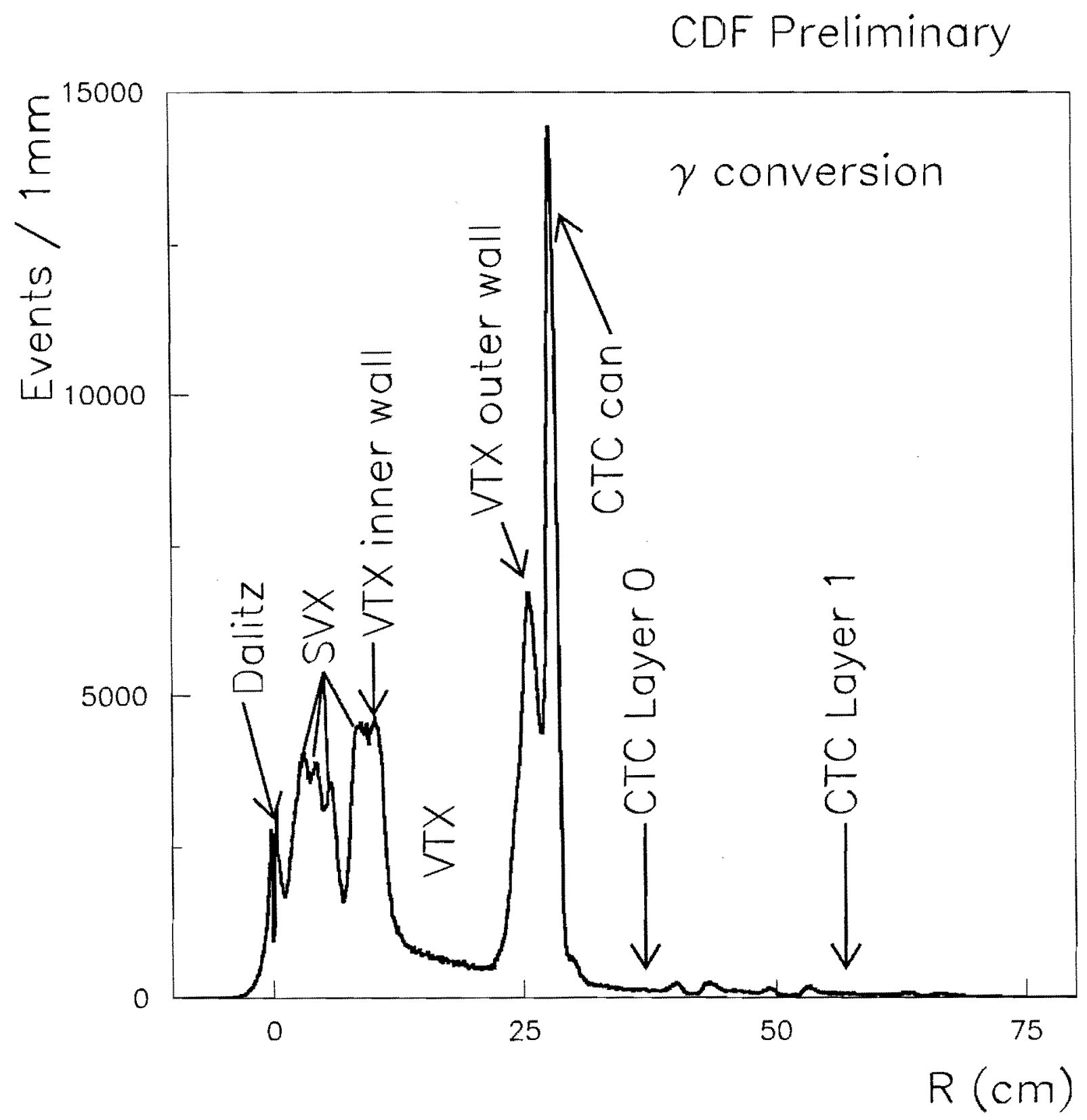

Figure 3.8: Trigger $\gamma$ conversion candidates in Run Ib data. 
known materials before the CTC, and also require the distance to be greater than $3 \mathrm{~cm}$ to reject the Dalitz background. A $\bar{B}_{d}\left(\bar{B}_{s}\right)$ candidate is formed by the $\gamma$ conversion candidate and a pair of oppositely charged tracks. Table 3.4 shows the reconstruction criteria for these channels. The meson tracks are required to be reconstructed as SVX tracks with at least 3 layer hits, and $p_{T}$ of each track and the two-track system must be greater than $0.5 \mathrm{GeV} / c$ and $2.0 \mathrm{GeV} / c$, respectively. A fit is performed with constraints that the meson tracks are coming from a common vertex, the $\gamma$ conversion candidate points back to the strange meson decay vertex, and the four-track system points back to a primary vertex. We use the primary vertex closest to the $z$ position of the trigger electron track extrapolated to the beam line. The CL of the fit is required to be greater than $0.1 \%$. The $\bar{B}_{d}\left(\bar{B}_{s}\right)$ candidates passing a cut on the $\bar{K}^{* 0}(\phi)$ mass window of 80 (10) $\mathrm{MeV} / c^{2}$ around the world average value of $M_{K^{* 0}}=896.1 \mathrm{MeV} / c^{2}\left(M_{\phi}=1019.4\right.$ $\mathrm{MeV} / c^{2}$ ) are accepted. This mass window corresponds to about $1.5(2)$ times the width of $\Gamma_{K^{* 0}}=50.5 \mathrm{MeV} / c^{2}\left(\Gamma_{\phi}=4.4 \mathrm{MeV} / c^{2}\right)$. Both $K^{-} \pi^{+}$and $\pi^{-} K^{+}$combinations are considered for each $\bar{K}^{* 0}$ candidate. We choose the mass assignment closer to the world average value of $M_{K^{* 0}}$.

We also require $\left|\eta_{B}\right|<1.0, c \tau^{*}>100 \mu \mathrm{m}$, and $I_{B}>0.7$. The definition of $c \tau^{*}$, the proper decay length, is $c \tau^{*} \equiv L_{T B} \times \frac{p_{T B}}{M_{B}}$, where the $L_{T B}, p_{T B}$, and $M_{B}$ are the decay length in the transverse plane, the transverse momentum, and the reconstructed mass of the $B$ meson candidate, respectively. The typical $c \tau^{*}$ resolution is $30 \mu \mathrm{m}$. The definition of $I_{B}$, the $B$ isolation, is $I_{B} \equiv p_{T B} /\left(p_{T B}+\sum_{R \leq 1.0} p_{T}\right)$, where the sum is over tracks within a cone of $R=1.0$ in the $\eta-\phi$ space around the direction of the $B$ meson candidate.

Figure 3.9 shows the $\bar{B}_{d}$ and $\bar{B}_{s}$ mass distributions. There are 15 events of $\bar{B}_{d}$ and 1 event of $\bar{B}_{s}$ candidates in the $\pm 100 \mathrm{MeV} / c^{2}$ of $\bar{B}_{d}\left(5279.2 \mathrm{MeV} / c^{2}\right)$ and $\bar{B}_{s}(5369.3$ $\mathrm{MeV} / c^{2}$ ) mass windows, respectively, after the selection. The $B$ meson mass resolution is $45 \mathrm{MeV} / \mathrm{c}^{2}$ which is dominated by the momentum resolution of the trigger electron. The mass distribution has a tail toward lower mass region due to the photon bremsstrahlung of the electron. In Section 3.10, we further optimize the selection cuts to improve the sensitivity to the radiative $\bar{B}_{d}$ and $\bar{B}_{s}$ decays.

\subsection{2 $\Lambda \rightarrow p \pi$}

A $\Lambda$ candidate is formed by a pair of oppositely charged tracks. Since the $\Lambda$ has a long lifetime ( $c \tau \sim 8 \mathrm{~cm}$ ), it decays outside the SVX fiducial volume $\sim 85 \%$ of the time, and thus only $15 \%$ of the $\Lambda$ decays are expected to have associated SVX tracks. 
Table 3.4: Reconstruction criteria for the radiative $B$ decays.

\begin{tabular}{|l|ll|}
\hline & $\bar{K}^{* 0} \gamma$ & $\phi \gamma$ \\
\hline Track & 2 CTC $(e e)$ & 2 CTC $(e e)$ \\
& $2 \operatorname{SVX}(K \pi)$ & $2 \operatorname{SVX}(K K)$ \\
\hline$p_{T}$ & $p_{T(K, \pi)} \geq 0.5$ & $p_{T(K)} \geq 0.5$ \\
$(\mathrm{GeV} / \mathrm{c})$ & $p_{T(K \pi)} \geq 2.0$ & $p_{T(K K)} \geq 2.0$ \\
\hline fitting prob & $\geq 0.1 \%$ & $\geq 0.1 \%$ \\
\hline$\left|\eta_{B}\right|$ & $\leq 1.0$ & $\leq 1.0$ \\
\hline Mass & $\left|M_{K \pi}-M_{K^{* 0}}\right| \leq 80\left(\mathrm{MeV} / c^{2}\right)$ & $\left|M_{K K}-M_{\phi}\right| \leq 10\left(\mathrm{MeV} / c^{2}\right)$ \\
& $\left|M_{K \pi}-M_{K^{* 0}}\right| \leq\left|M_{\pi K}-M_{K^{* 0}}\right|$ \\
\hline Vertex & $\sigma L_{X Y} \leq 300 \mu \mathrm{m}$ & $\sigma L_{X Y} \leq 300 \mu \mathrm{m}$ \\
& $c \tau^{*} \geq 100 \mu \mathrm{m}$ & $c \tau^{*} \geq 100 \mu \mathrm{m}$ \\
\hline$B$ isolation $\dagger$ & $I_{B} \geq 0.7$ & $I_{B} \geq 0.7$ \\
\hline
\end{tabular}

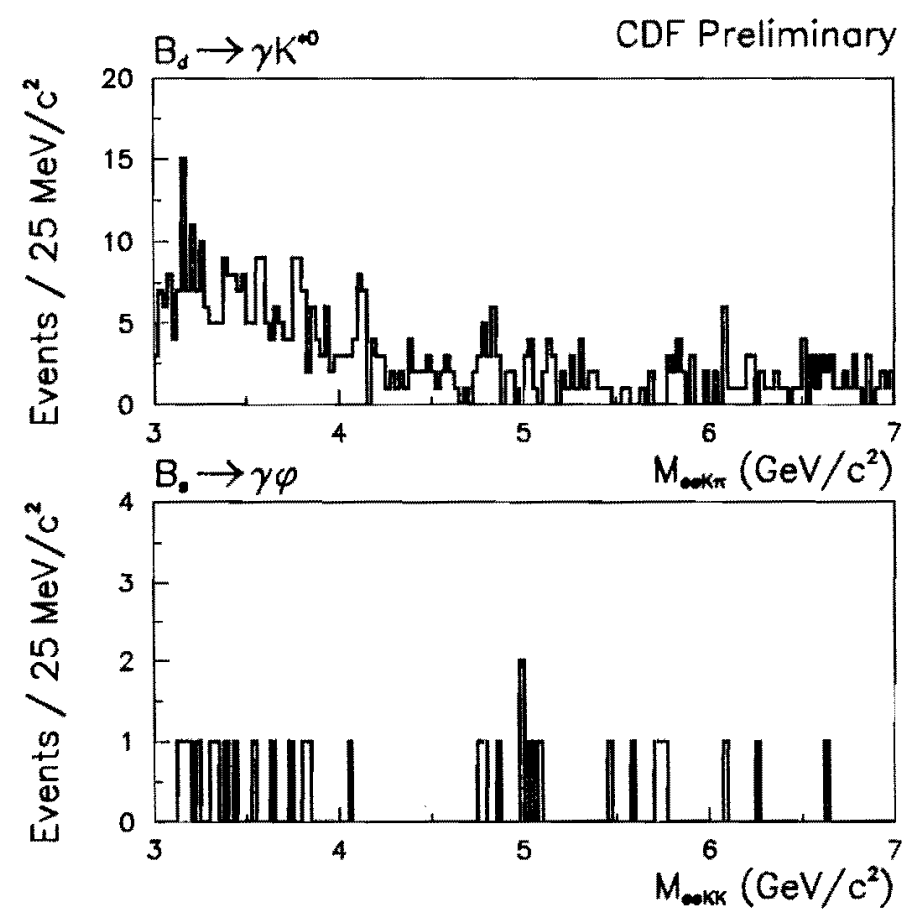

Figure 3.9: $\bar{B}_{d} \rightarrow \bar{K}^{* 0} \gamma$ and $\bar{B}_{s} \rightarrow \phi \gamma$ mass distribution in Run IB data. 
Then we classify the $\Lambda$ candidates into two categories: (i) "CTC- $\Lambda$ ", consisting of $\Lambda$ 's reconstructed with CTC tracks for both the proton and pion; and (ii) "SVX- $\Lambda$ ", which is a subset of the first, containing events with SVX information for the tracks.

We first reconstruct CTC- $\Lambda$ 's. The mass assignment of the tracks is determined by assuming the $p_{T}$ of the proton track to be higher than that of the pion track. The proton and pion tracks are required to have $p_{T}>1.5 \mathrm{GeV} / c$ and $p_{T}>0.4 \mathrm{GeV} / c$, respectively, and a measured $d E / d x$ consistent with expectations. We define the $d E / d x$ cut parameter as follows,

$$
f(d E / d x)=\frac{d E / d x(p, p) \times d E / d x(\pi, \pi)}{d E / d x(p, e) \times d E / d x(\pi, e)}
$$

and $d E / d x(p, p)$ is $d E / d x$ of the track assigned as a proton with a proton assumption (See Equation 2.2), and $d E / d x(\pi, e)$ is $d E / d x$ of the track assigned as a pion with an electron assumption (See Equation 2.2), and The cut parameter is small for $\Lambda \rightarrow p \pi$ and large for $\gamma$ conversions. We require $f(d E / d x)<0.2$.

Track pairs with an invariant mass inferior to $2 \mathrm{GeV} / \mathrm{c}^{2}$ are then fit under a common vertex constraint, and accepted if the CL of the fit is greater than $0.1 \%$. One of the major backgrounds in reconstructing $\Lambda \rightarrow p \pi^{-}$decays is the photon conversion. By performing the same constrained-fit applied in the photon conversion identification, we reject the track pair that is consistent with such hypothesis. Finally, the $\Lambda$ candidate is accepted, as a CTC- $\Lambda$ candidate, if the distance of the $\Lambda$ decay vertex from the nominal beamline is greater than $1 \mathrm{~cm}$.

If both the proton and the pion are SVX tracks with at least 2 SVX layers hit, the common vertex constraint fit is redone, using the SVX track information this time. Again, the CL of the fit must be greater than $0.1 \%$. This time, we also require the SVX layer hit pattern to be consistent with the expectation from the reconstructed $\Lambda$ decay. For example, if the $\Lambda$ decay vertex is between the SVX layers 2 and 3 , we require the tracks to have exactly 2 hits in the SVX layers 3 and 4 (See Figure 3.10). The average radius of each the SVX layer is given as $3.08,4.37,5.84$, and 8.07 , for L1, 2, 3, and 4, respectively. We require: i) at least 3 hits for the tracks with $R_{\Lambda}<R_{L 1}$; ii) at least 2 hits and no hit in L1 for $R_{L 1}<R_{\Lambda}<R_{L 2}$; iii) exactly 2 hits in the L3 and L4 for $R_{L 2}<R_{\Lambda}<R_{L 3}$. The $\Lambda$ candidates with $R_{L 3}<R_{\Lambda}$ is rejected.

A $10 \%$ of the CTC- $\Lambda$ candidates satisfy the above requirements and are thus classified as SVX- $\Lambda$ candidates. 


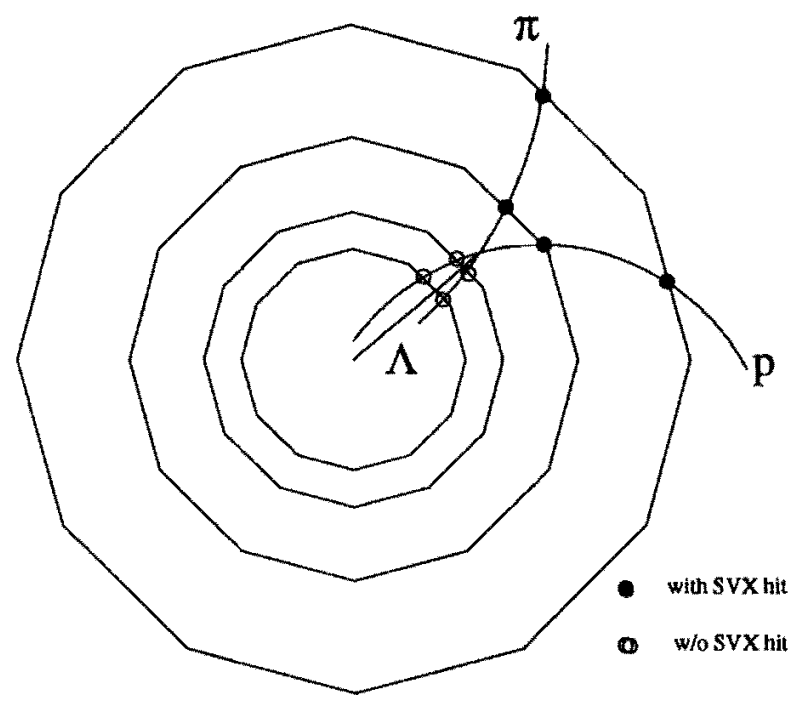

Figure 3.10: $\Lambda \rightarrow p \pi$ reconstruction in the SVX.

\subsection{3 $\Lambda_{b} \rightarrow \Lambda \gamma$}

A $\Lambda_{b}$ candidate is formed by a photon conversion and a $\Lambda$ candidate. For the CTC$\Lambda$ candidates, we reconstruct $\Lambda_{b}$ with a constraint that both the $\Lambda$ and the photon conversion point back to the primary vertex. This constraint significantly improves the resolution of the reconstructed $\Lambda_{b}$ mass; the typical resolutions are 50 and $75 \mathrm{MeV} / \mathrm{c}^{2}$ with and without the constraint, respectively (See Figure 3.11. On the other hand, for the SVX- $\Lambda$ candidates, only the photon conversion is constrained to point back to the primary vertex, while the $\Lambda$ mometum, extrapolated to the beamline, is required to be within $2 \mathrm{~cm}$ from the primary vertex in the $z$ direction. The typical $\Lambda_{b}$ mass resolution for the SVX- $\Lambda$ is $50 \mathrm{MeV} / \mathrm{c}^{2}$. In both cases, we require the CL of the constrained fit to be greater than $0.1 \%$.

We then calculate the resulting $\Lambda$ mass after the fit, and the $\Lambda \gamma$ combination, called either a CTC- $\Lambda_{b}$ or an SVX- $\Lambda_{b}$ candidate depending on the $\Lambda$ category, is accepted if the $p \pi$ mass is within $\pm 3 \mathrm{MeV} / \mathrm{c}^{2}$ from the world average $\Lambda$ mass [6]. The typical $\Lambda$ mass resolutions are 2.5 and $1.5 \mathrm{MeV} / \mathrm{c}^{2}$ for the $\mathrm{CTC}-\Lambda_{b}$ and $S V X-\Lambda_{b}$ candidates, respectively.

We improve the sample purity by requiring the impact parameter significances for the proton and pion tracks to be larger than 0.5 (3.0) and $2.0(3.0)$, respectively, and the decay length of the $\Lambda$ in the transverse plane to be larger than $3.0 \mathrm{~cm}(1.0 \mathrm{~cm})$, for the CTC- $\Lambda_{b}\left(\mathrm{SVX}-\Lambda_{b}\right)$ candidates. Finally, we require $\left|\eta_{\Lambda_{b}}\right|<1$ and $I_{B}>0.7$. Figure 3.12 shows the $\Lambda$ and $\Lambda_{b}$ mass distribution after the selection cuts. we find 23 candidates of CTC- $\Lambda_{b}$ and 2 candidates of $S V X-\Lambda_{b}$ in the $\pm 100 \mathrm{MeV} / \mathrm{c}^{2}$ window around the world 


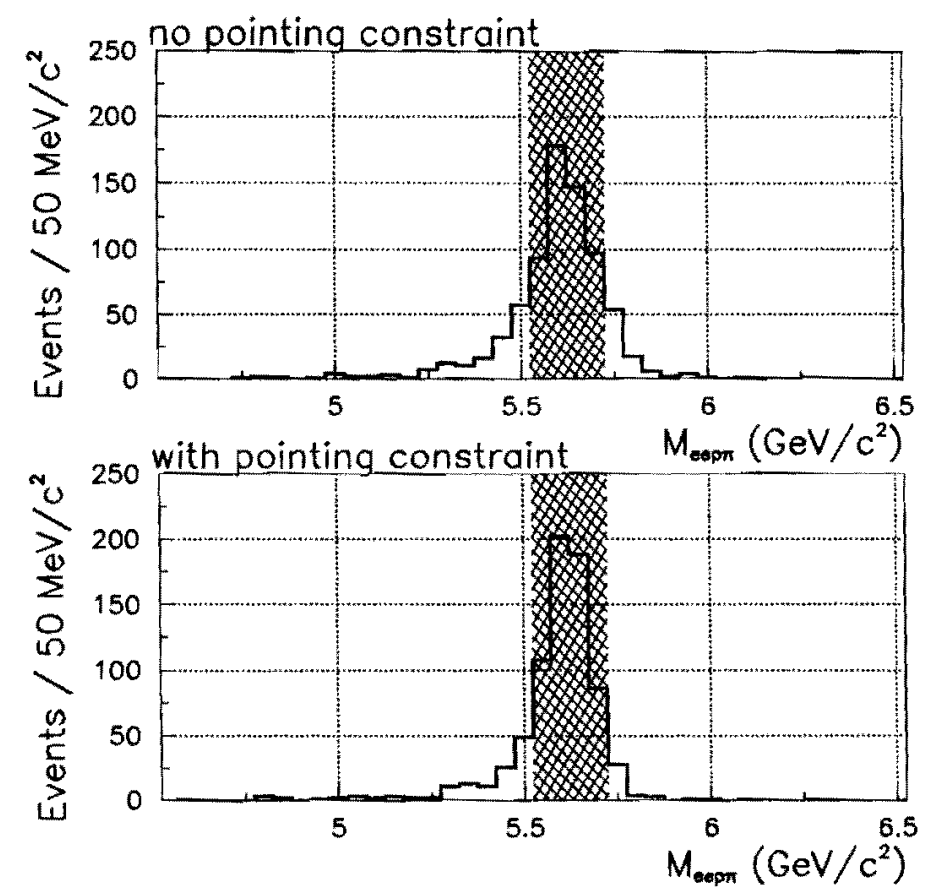

Figure 3.11: $M_{\Lambda_{b}}$ distribution for the signal $\mathrm{MC}$ before and after the constraint fit that both the $\gamma$ and $\Lambda$ point back to the primary vertex.

average $\Lambda_{b}$ mass. Further fine tuning of the selection cuts to maximize the sensitivity is discussed in Section 3.10.

\section{9 $\bar{B} \rightarrow J / \psi$ Decay Reconstruction}

\subsection{1 $\bar{B}_{u} \rightarrow J / \psi K^{-}$}

For each $J / \psi \rightarrow$ ee candidate in an event we search for $\bar{B}_{u} \rightarrow J / \psi K^{-}$. We first require the electron tracks to have at least 3 SVX hits. A $\bar{B}_{u}$ candidate is formed by the $J / \psi$ candidate and an SVX track with at least $3 \mathrm{SVX}$ hits and $p_{T}>2.0 \mathrm{GeV} / c$. We constrain the three tracks to have a common vertex and a total momentum pointing back to the primary vertex is made We accept the three-track combination if the CL of this constraint fit is greater than $0.1 \%$. The $\bar{B}_{u}$ candidates passing the cuts of $\left|\eta_{B}\right|<$ $1.0, c r^{*}>100 \mu \mathrm{m}$, and $I_{B}>0.7$ are accepted.

The $M(e e K)$ distribution has a tail towards low mass values due to the effect of the photon bremsstrahlung on the electron tracks. In order to correct for it and, at the same time, compensate for the resolution loss due to the electron momentum uncertainty, we use $M(e e K)-M(e e)+M(J / \psi)$ instead of $M(e e K)$, where $M(J / \psi)$ is the world average $J / \psi$ mass. Figure 3.13 shows scatter plots between the $M(e e K)$ and 

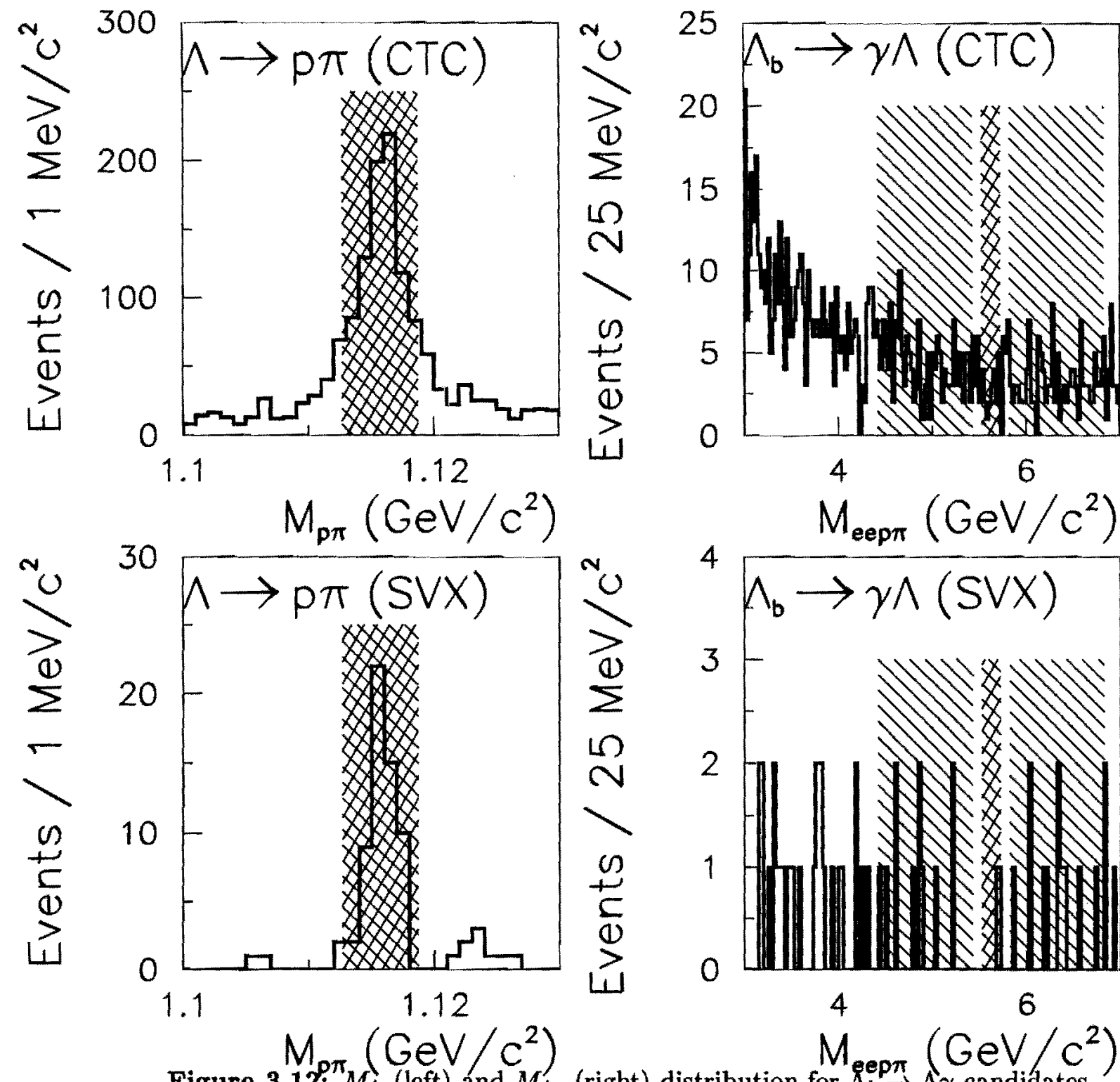

CDF Preliminary

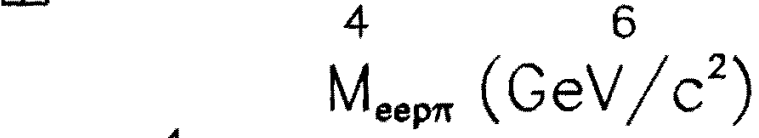


Table 3.5: Reconstruction criteria for the $\Lambda_{b} \rightarrow \Lambda \gamma$.

\begin{tabular}{|c|c|c|}
\hline & $\mathrm{CTC}-\Lambda_{b}$ & $S V X-\Lambda_{b}$ \\
\hline Track & $4 \mathrm{CTC}(e e p \pi)$ & 2 CTC $(e e) 2 \operatorname{SVX}(p \pi)$ \\
\hline \multirow{4}{*}{$\begin{array}{l}p_{T} \\
(\mathrm{GeV} / \mathrm{c})\end{array}$} & \multicolumn{2}{|c|}{$p_{T(p \pi)}>2.0$} \\
\hline & \multicolumn{2}{|c|}{$p_{T(p)}>p_{T(\pi)}$} \\
\hline & \multicolumn{2}{|c|}{$p_{T(p)}>1.5$} \\
\hline & \multicolumn{2}{|c|}{$p_{T(\pi)}>0.4$} \\
\hline$d E / d x$ & \multicolumn{2}{|c|}{$|f(d E / d x)|<0.2$} \\
\hline fitting prob & \multicolumn{2}{|c|}{$>0.1 \%$} \\
\hline$\left|\eta_{B}\right|$ & \multicolumn{2}{|r|}{$<1.0$} \\
\hline Mass & \multicolumn{2}{|c|}{$\left|M_{p \pi}-M_{\Lambda}\right|<3\left(\mathrm{MeV} / c^{2}\right)$} \\
\hline \multirow[t]{4}{*}{ Vertex } & $3 \mathrm{~cm}<L_{X Y}(\Lambda)$ & $1 \mathrm{~cm}<L_{X Y}(\Lambda)$ \\
\hline & $d / \sigma(p)>0.5$ & $d_{0} / \sigma(p)>3.0$ \\
\hline & $d / \sigma(\pi)>2.0$ & $d_{0} / \sigma(\pi)>3.0$ \\
\hline & \multicolumn{2}{|c|}{$3.0<L_{X Y}(\gamma)<30.0 \mathrm{~cm}$} \\
\hline$B$ isolation & \multicolumn{2}{|c|}{$I_{B} \geq 0.7$} \\
\hline Optimization & $p_{T \Lambda}>4.0 \mathrm{GeV} / c$ & $d_{0 \Lambda}>70 \mu \mathrm{m}$ \\
\hline
\end{tabular}

$M(e e)$. The typical mass resolutions are $50 \mathrm{MeV} / c^{2}$ and $25 \mathrm{MeV} / c^{2}$ for $M(e e K)$ and $M(e e K)-M(e e)+M(J / \psi)$, respectively. The bottom plot of Figure 3.14 shows the mass distribution for the $\bar{B}_{u} t o J / \psi K^{-}$candidates. We obtain about $50 \bar{B}_{u}$ candidates with $S / B \simeq 10$ in the mass window of $\pm 100 \mathrm{MeV} / c^{2}$ arround the $B_{u}\left(5278.9 \mathrm{MeV} / c^{2}\right)$.

Figure 3.15 shows comparison of the data (points) and MC (histograms) several reconstructed parameters: the corrected $\bar{B}_{u}$ mass, momentum of $\bar{B}_{u}$, dielectron mass, electron $E_{T}$, proper decay time, and impact parameter of $K^{-}$. The MC reproduces all the parameters of the data very well.

\subsection{2 $\bar{B}_{d} \rightarrow J / \psi \bar{K}^{* 0}$}

Reconstruction of the $\bar{B}_{d} \rightarrow J / \psi \bar{K}^{* 0}$ uses the same $J / \psi \rightarrow$ ee selection criteria as $\bar{B}_{u} \rightarrow J / \psi K^{-}$and the same $\bar{K}^{* 0}$ selection criteria as $\bar{B}_{d} \rightarrow \bar{K}^{* 0} \gamma$. For each $J / \psi \rightarrow e e$ candidate in an event we search for $\bar{B}_{d} \rightarrow J / \psi \bar{K}^{* 0}$. We require all the four tracks to have at least $3 \mathrm{SVX}$ hits. We also require the transeverse momenta of each meson track the two-meson system must be greater than $0.5 \mathrm{GeV} / c$ and $2.0 \mathrm{GeV} / c$, respectively.

A fit is performed with constraints that all the electron and meson tracks are coming from a common vertex, and the four-track system points back to a primary vertex. We 

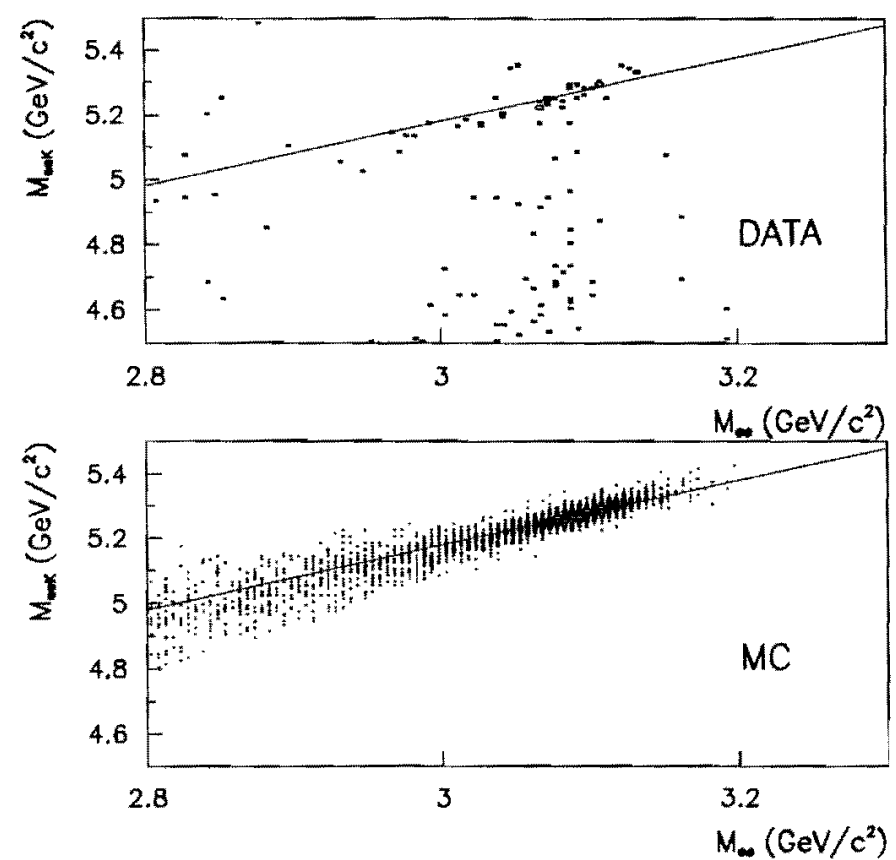

Figure 3.13: Correlation between of $M_{e e K}$ and $M_{e e}$ for the $J / \psi K$ candidates (top) and signal MC (bottom). The line shows $M_{e e K}-M_{e e}=M_{B_{u}}-M_{J / \psi}$.
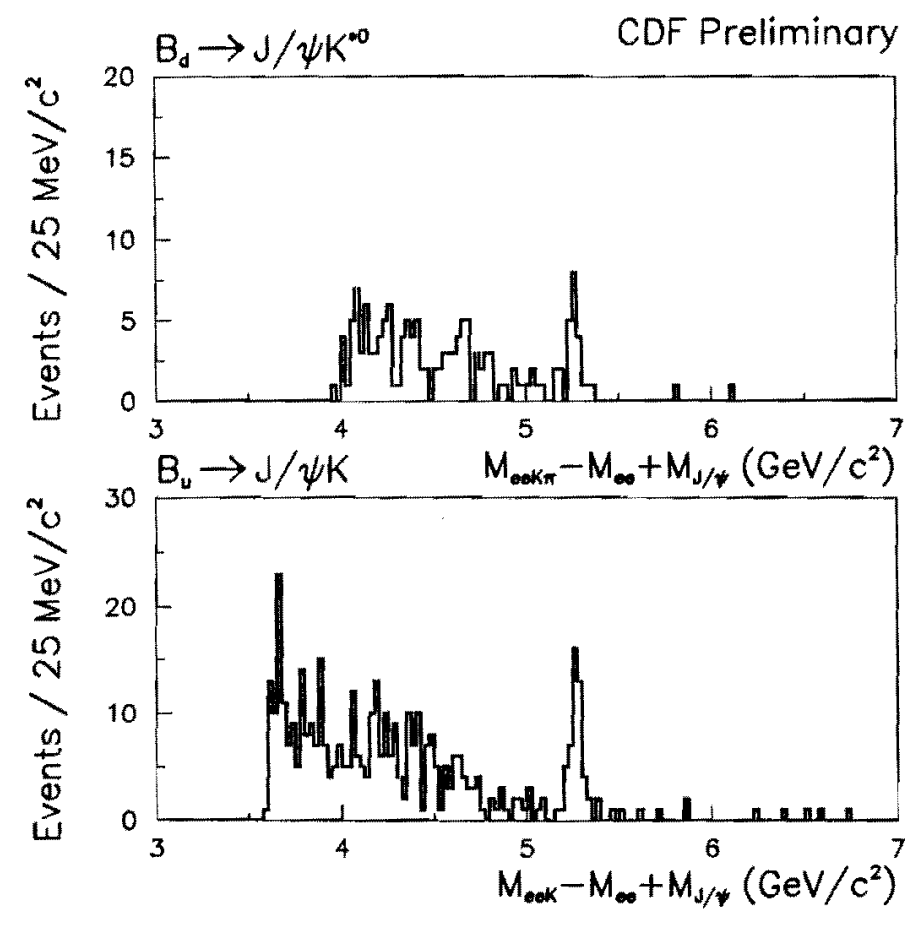

Figure 3.14: $\bar{B}_{d} \rightarrow J / \psi \bar{K}^{* 0}$ and $\bar{B}_{u} \rightarrow J / \psi K^{-}$mass distribution in Run Ib data. 


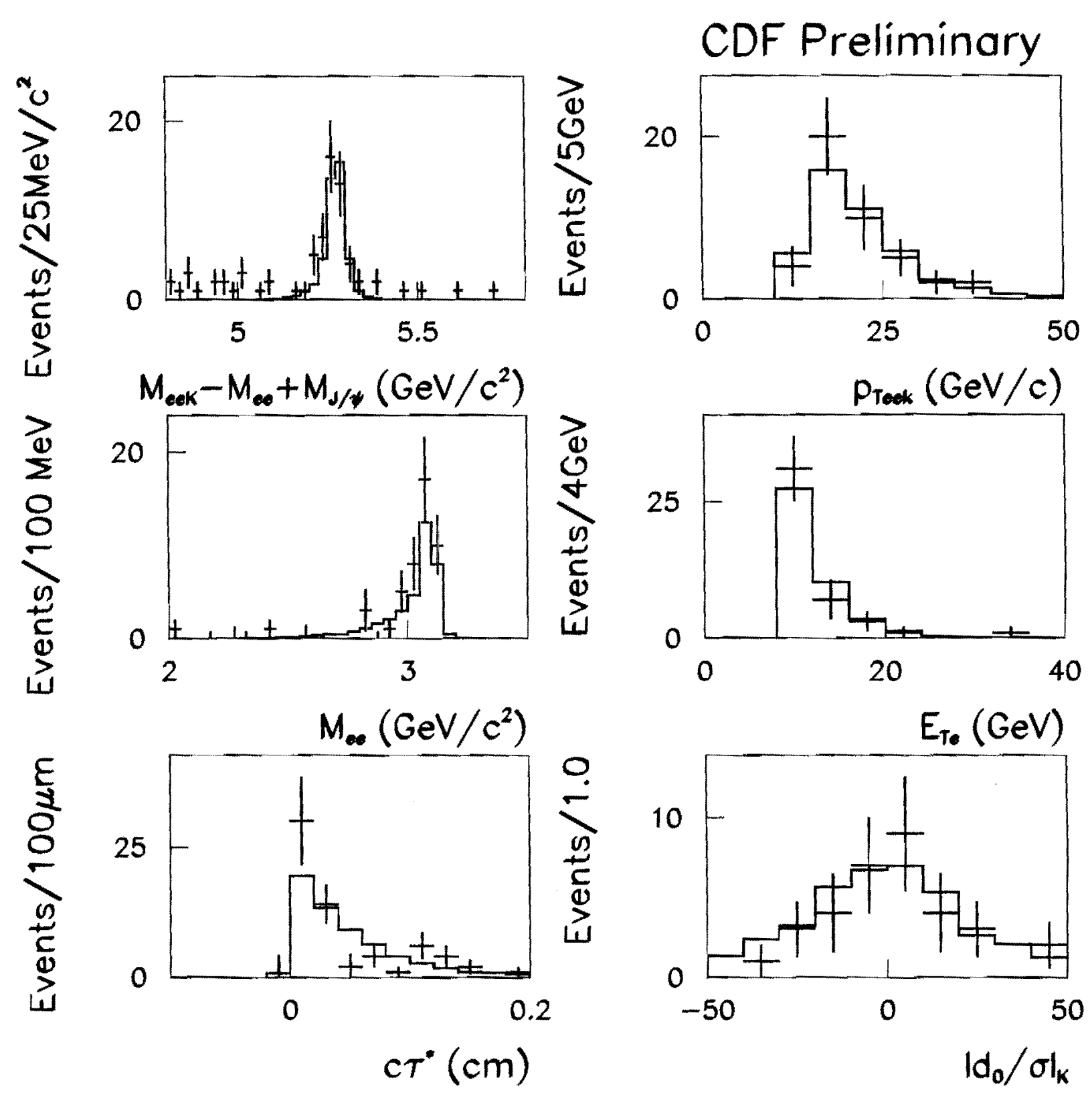

Figure 3.15: Comparison of the data (points) and $\mathrm{MC}$ (histograms) for the $\bar{B}_{u} \rightarrow J / \psi K^{-}$candidates in several reconstructed parameters. 
use the primary vertex closest to the $z$ position of the trigger electron track extrapolated to the beam line. The CL of the fit is required to be greater than $0.1 \%$. The $\bar{B}_{d}$ candidates passing a cut on the $\bar{K}^{* 0}$ mass window of $80 \mathrm{MeV} / c^{2}$ around the world average value of $M_{K^{* 0}}=896.1 \mathrm{MeV} / c^{2}$ are accepted. Both $K^{-} \pi^{+}$and $\pi^{-} K^{+}$combinations are considered for each $\bar{K}^{* 0}$ candidate. We choose the mass assignment closer to the world average value of $M_{K^{* 0}}$. The $\bar{B}_{d}$ candidates passing the cuts of $\left|\eta_{B}\right|<1.0, c \tau^{*}>100 \mu \mathrm{m}$, and $I_{B}>0.7$ are accepted.

The top plot of Figure 3.14 shows the $M(e e K \pi)-M(e e)+M(J / \psi)$ distribution for the $\bar{B}_{d} \rightarrow J / \psi \bar{K}^{* 0}$ candidates. We obtain about $20 \bar{B}_{d}$ candidates with $S / B \simeq 10$ mass window of $\pm 100 \mathrm{MeV} / c^{2}$ around the $B_{u}\left(5278.9 \mathrm{MeV} / c^{2}\right)$.

\subsection{Cut Optimization}

To improve our sensitivity to the radiative decays we optimize the selection cut by maximizing the figure of merit $($ fom $)$ defined as fom $=\epsilon_{s i g} / \sqrt{\epsilon_{b g}}$, where $\epsilon_{s i g}$ and $\epsilon_{b g}$ is the efficiency for the signal and background events, respectively, in the $\pm 100 \mathrm{MeV} / \mathrm{c}^{2}$ of the $B$-hadron mass window.

The $\epsilon_{s i g}$ is estimated from the MC sample. The $\epsilon_{b g}$ is estimated by extrapolating the observed number of events in $B$-hadron mass sideband, $\pm(200$ to 1200$) \mathrm{MeV} / c^{2}$, to the signal mass regions. We apply similar selection cuts to the $\bar{B}_{u} \rightarrow J / \psi K^{-}$decay as the radiative $B$-hadron decays to achieve the cancelation of systematic uncertainties as much as possible.

For the $\bar{B}_{d}$ channel, we choose $p_{T}(K \pi)$ and $d(K, \pi) / \sigma$ for the optimization, and similarly, $p_{T}(K K)$ and $d(K) / \sigma$ for $\bar{B}_{s}$, where $\sigma$ is the uncertainty on the impact parameter $d$. We apply the same cut values obtained for the $\bar{B}_{d}$ or $\bar{B}_{s}$ decay to $p_{T}(K)$ and $d(K) / \sigma$ for the reference channel of the $\bar{B}_{u} \rightarrow J / \psi K^{-}$decay. Table 3.6 shows the fom around the optimized set of the selection cut parameters. The optimized set of selection cuts for the $\bar{B}_{d}$ channel is $p_{T}(K \pi)>2.75 \mathrm{GeV} / c$ and $|d(K, \pi) / \sigma|>4.5$. Figure 3.16 shows the $\bar{K}^{* 0} \gamma$ and $J / \psi K$ mass distributions after the cuts. Any further cuts, for example, on the proper decay length, do not improve the figure of merit. There is one candidate in the $\bar{B}_{d}$ signal region with expected backgrounds of $0.6 \pm 0.3$ events, while we find $28.0 \pm 5.8 \bar{B}_{u} \rightarrow J / \psi K^{-}$candidates in the $\pm 100 \mathrm{MeV} / \mathrm{c}^{2}$ mass window after subtracting the backgrounds estimated from the side bands, $\pm(200$ to 300$) \mathrm{MeV} / \mathrm{c}^{2}$. Figure 3.17 shows the event display in the CTC for the candidate. It corresponds the $B_{d} \rightarrow K^{* 0} \gamma \rightarrow K^{-} \pi^{+} e^{+} \epsilon^{-}$where the photon conversion point is found in the CTC inner 
Table 3.6: Cut optimization for $\bar{B}_{d} \rightarrow \bar{K}^{* 0} \gamma$.

\begin{tabular}{lllllll}
\hline \hline$p_{T}(K \pi)$ & $c \tau^{*}$ & $d / \sigma(K), d / \sigma(\pi)$ & $N_{B G}$ & fom & $N_{K^{*} \gamma}$ & $N_{J / \psi K}$ \\
\hline $2.25(\mathrm{GeV} / \mathrm{c})$ & $100(\mu \mathrm{m})$ & 4.5 & 1.0 & 5.1 & 1 & 33 \\
2.75 & 100 & $\underline{4.5}$ & 0.6 & 5.9 & 1 & 28 \\
3.0 & 100 & 4.5 & 0.6 & 5.7 & 1 & 27 \\
\hline 2.75 & 100 & 4.5 & 0.6 & 5.9 & 1 & 28 \\
2.75 & 150 & 4.5 & 0.6 & 5.7 & 1 & 27 \\
2.75 & 200 & 4.5 & 0.6 & 5.3 & 1 & 25 \\
\hline 2.75 & 100 & 3.0 & 1.0 & 4.8 & 2 & 29 \\
2.75 & 100 & 4.5 & 0.6 & 5.9 & 1 & 28 \\
2.75 & 100 & 6.0 & 0.6 & 5.3 & 1 & 27 \\
\hline
\end{tabular}

Table 3.7: Cut optimization for $\bar{B}_{s} \phi \phi \gamma$.

\begin{tabular}{lllllll}
\hline \hline$p_{T}(K K)$ & $c \tau^{*}$ & $d / \sigma(K)$ & $N_{B G}$ & fom & $N_{\phi \gamma}$ & $N_{J / \psi K}$ \\
\hline $2.0(\mathrm{GeV} / \mathrm{c})$ & $100(\mu \mathrm{m})$ & 3 & 0.3 & 3.0 & 0 & 36. \\
2.25 & $\underline{100}$ & $\underline{3}$ & 0.1 & 4.9 & 0 & 35. \\
2.5 & 100 & 3 & 0.0 & - & 0 & 30. \\
\hline 2.25 & 100 & 3 & 0.1 & 4.9 & 0 & 35. \\
2.25 & 125 & 3 & 0.1 & 4.7 & 0 & 34. \\
2.25 & 150 & 3 & 0.1 & 4.5 & 0 & 33. \\
\hline 2.25 & 100 & 1.5 & 0.6 & 2.1 & 0 & 41. \\
2.25 & 100 & 3 & 0.1 & 4.9 & 0 & 35. \\
2.25 & 100 & 4.5 & 0.1 & 4.6 & 0 & 33. \\
\hline
\end{tabular}

wall and the $e^{-}$is used as the trigger electron. The reconstructed $B_{d}$ and $K^{* 0}$ masses of 5.31 and $0.896 \mathrm{GeV} / c^{2}$, respectively, are consistent with the world average. Figure 3.18 shows the display close to the interaction point. The $K^{-}$and $\pi^{+}$form a significantly separated secondary vertex from the primary vertex, and estimated $B_{d}$ proper decay time is $591 \mu \mathrm{m}$

The optimized set of cuts for the $\bar{B}_{s}$ channel is $p_{T}(K K)>2.25 \mathrm{GeV} / c$ and $|d(K) / \sigma|>3.0$. The invariant mass distributions of the $\phi \gamma$ and $J / \psi K$ combinations serving the cuts are shown in Fig. 3.19. No candidates are found in the $\bar{B}_{s}$ signal region, where we expect a background of $0.1 \pm 0.1$ events, while $35.0 \pm 6.4 \bar{B}_{u} \rightarrow J / \psi K^{-}$decays are observed after background subtraction.

We perform the cut optimization separately for the CTC- $\Lambda_{b}$ and $S V X-\Lambda_{b}$ candidates. 

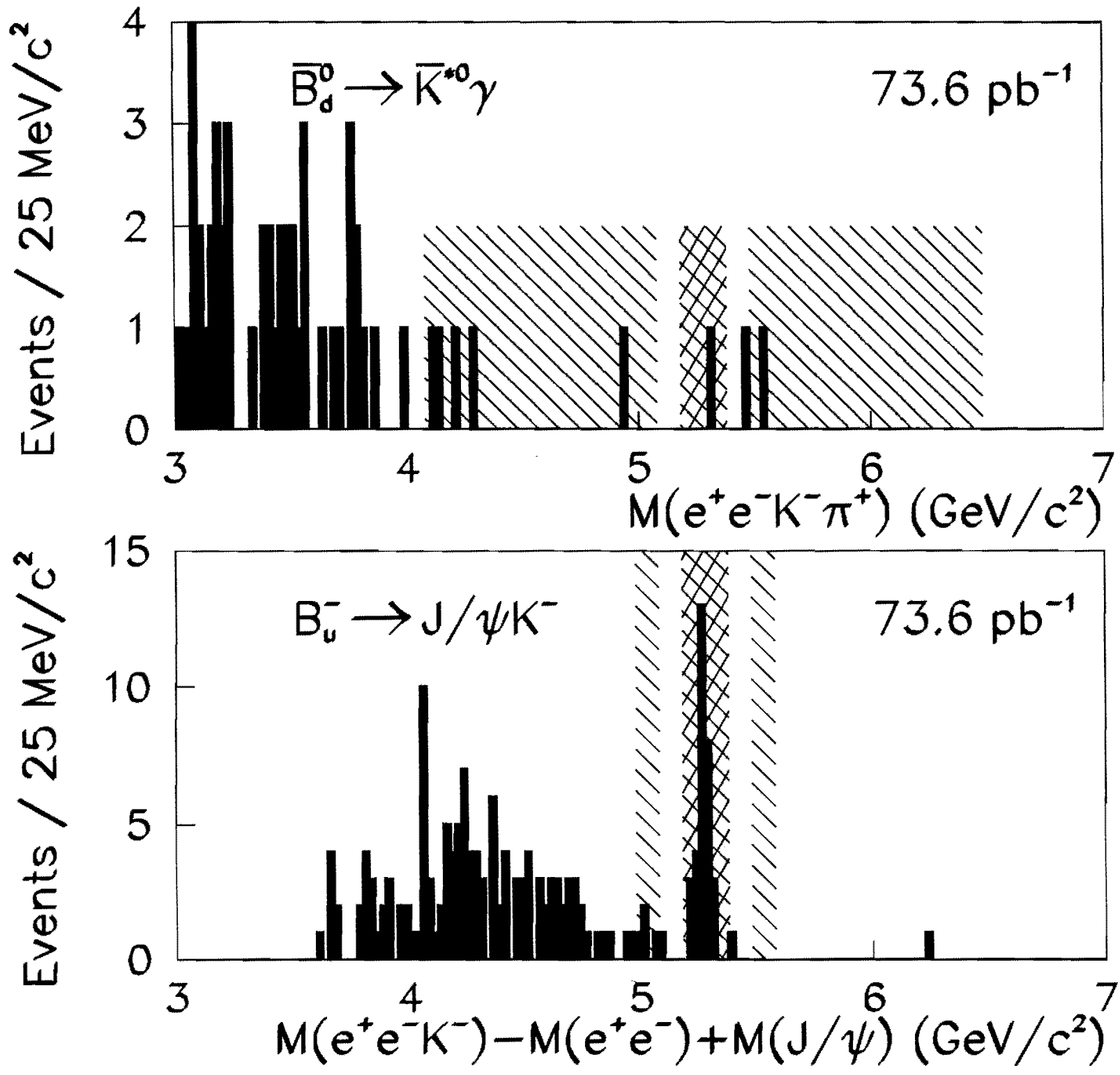

Figure 3.16: $\bar{K}^{* 0} \gamma$ and $J / \psi K^{-}$mass distribution after the optimized cut. 


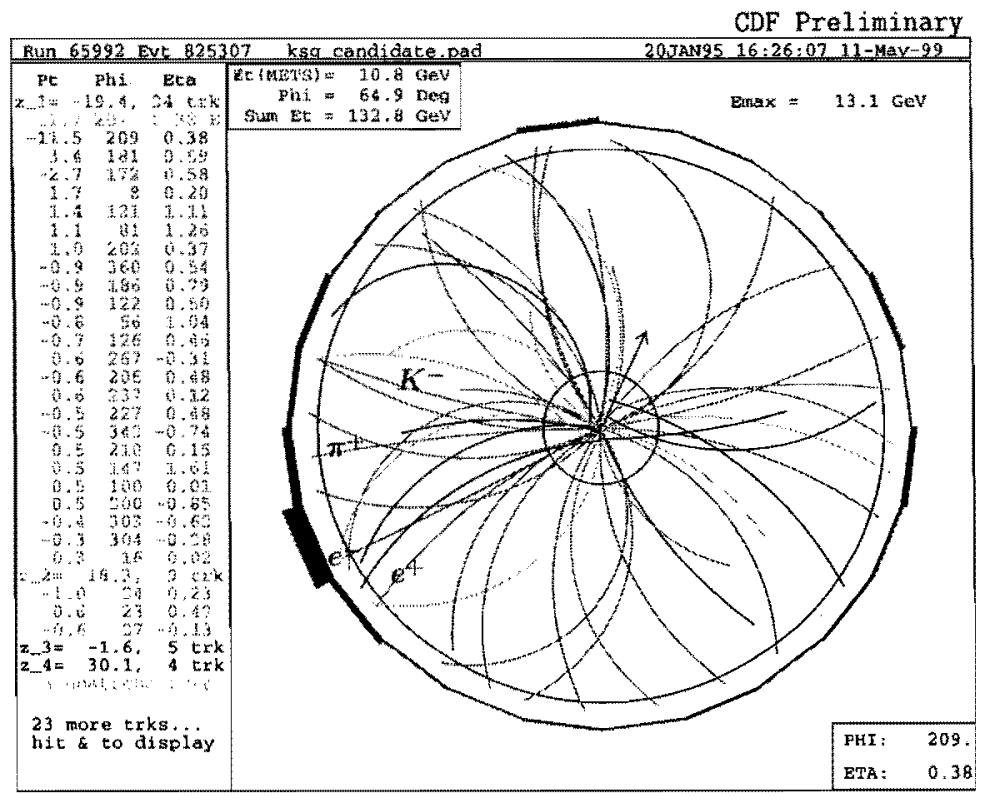

Figure 3.17: The CTC display of a $\bar{K}^{* 0} \gamma$ candidate.

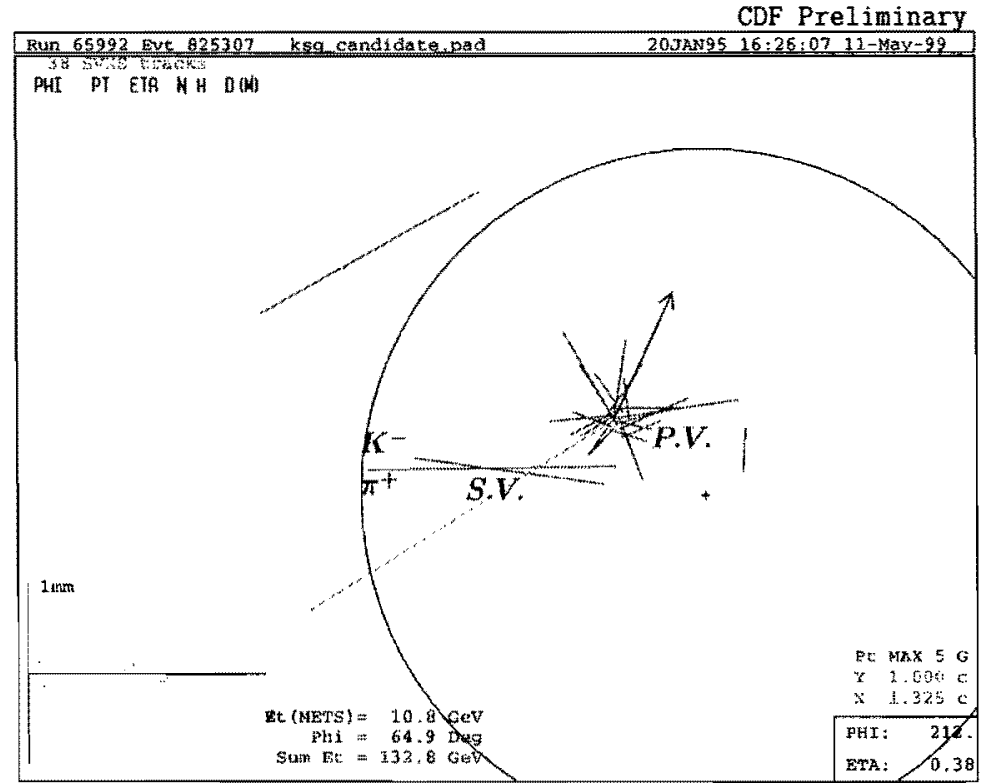

Figure 3.18: The SVX display of a $\bar{K}^{* 0} \gamma$ candidate. 


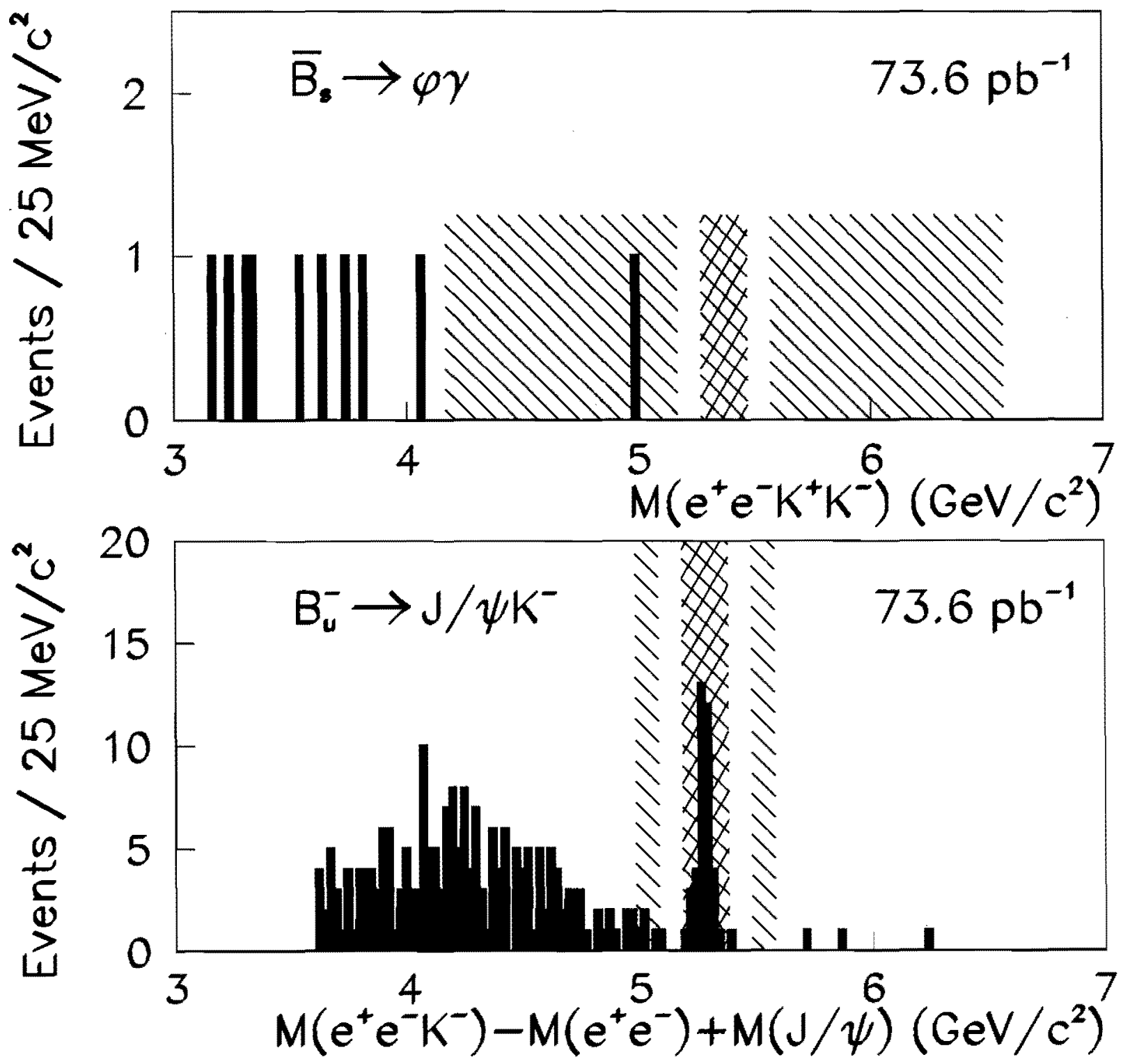

Figure 3.19: $\phi \gamma$ and $J / \psi K^{-}$mass distribution after the optimized cut. 
For the SVX $-\Lambda_{b}$ candidates, we consider the signed impact parameter of $\Lambda$. The sign is defined as positive when the crossing point of the reconstructd $\Lambda$ and the $\Lambda_{b}$ momenta is in the hemisphere containing the $\Lambda_{b}$, as should be the topology of real $\Lambda_{b}$ decays (Figure 3.20). Figure 3.21 shows the $\Lambda$ impact parameter distribution for the $\Lambda_{b}$ mass side band events in the data, and signal MC sample. The typical resolution of $d_{0 \Lambda}$ is $40 \mu \mathrm{m}$. Figure 3.23 shows the fom as a function of the $d_{0 \text { Llambda }}$ cut. The optimized cut value which is shown as lines in the plots is $70 \mu \mathrm{m}$. Figure 3.24 shows the $\Lambda$ and $\Lambda_{b}$ mass distributions for the candidates. Applying this cut results in no SVX- $\Lambda_{b}$ candidates in the signal region with an expected background of $0.1 \pm 0.1$ events.

The impact parameter resolution of the CTC tracks is not as good as the SVX tracks, thus, for the CTC- $\Lambda_{b}$ case, we optimize the $p_{T}(\Lambda)$.

we reinforce the kinematical requirement by applying $p_{T}(\Lambda)>4 \mathrm{GeV} / \mathrm{c}$. We find 2 candidates in the signal region, with an expected background of $3.3 \pm 0.6$ events. Figure 3.22 shows the $p_{T}(\Lambda)$ distribution for the $\Lambda_{b}$ mass side band events in the data (points), and signal MC sample (histogram). Figure 3.23 shows the fom as a function of the $p_{T}(\lambda)$ cut. The optimized cut value which is shown as lines in the plots is 4.0 $\mathrm{GeV} / c$. Figure 3.24 shows the $\Lambda$ and $\Lambda_{b}$ mass distributions for the candidates. We find 2 candidates in the signal region, with an expected background of $3.3 \pm 0.6$ events.

Adding the CTC and SVX- $\Lambda_{b}$ candidates, there are 2 candidates in the signal region with expected backgrounds of $3.4 \pm 0.6$ events. The reference sample of $\bar{B}_{u} \rightarrow J / \psi K^{-}$ decays is obtained by requiring $p_{T}(K)>4 \mathrm{GeV} / c$, and we find $24.0 \pm 5.3$ events after background subtraction. The invariant mass distributions are shown in Fig. 3.25 .

Since we do not find significant excesses over background for all the radiative decay modes (See Table 4.1), we set uppers limits on the branching fractions in the following chapters. 


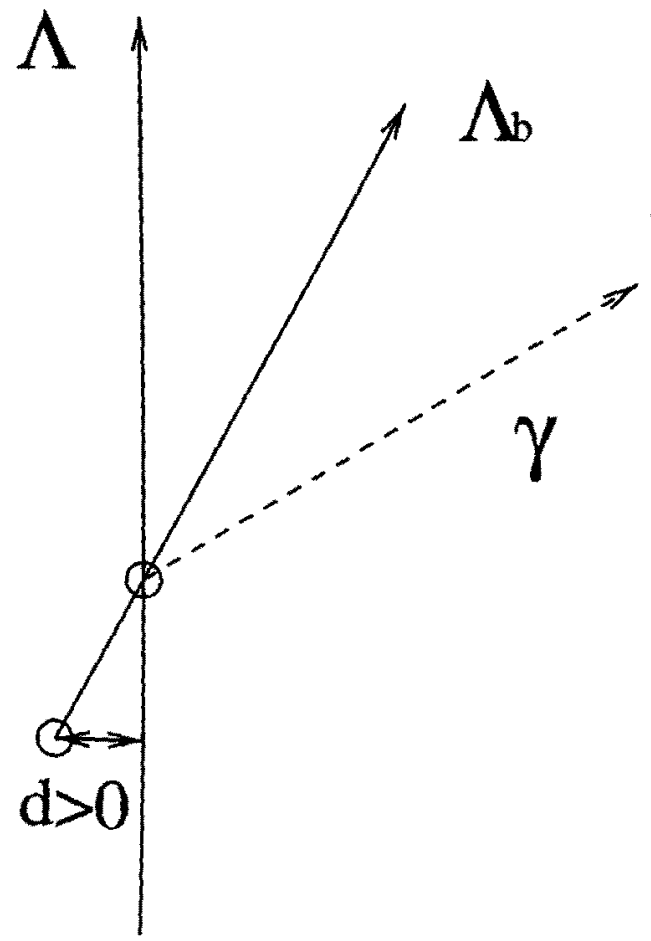

Figure 3.20: Definition of the signed impact parameter.
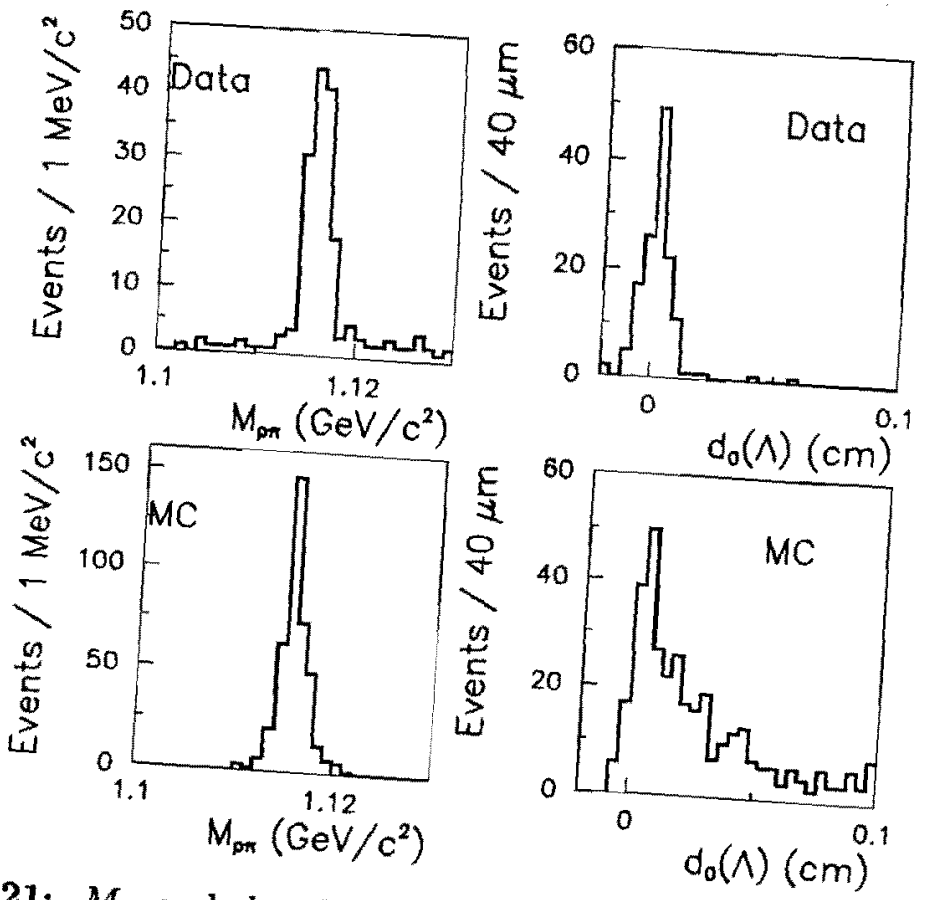

Figure 3.21: $M_{\Lambda}$ and $d_{0 \Lambda}$ distribution for the SVX $\Lambda_{b}$ candidates and the
signal MC. 


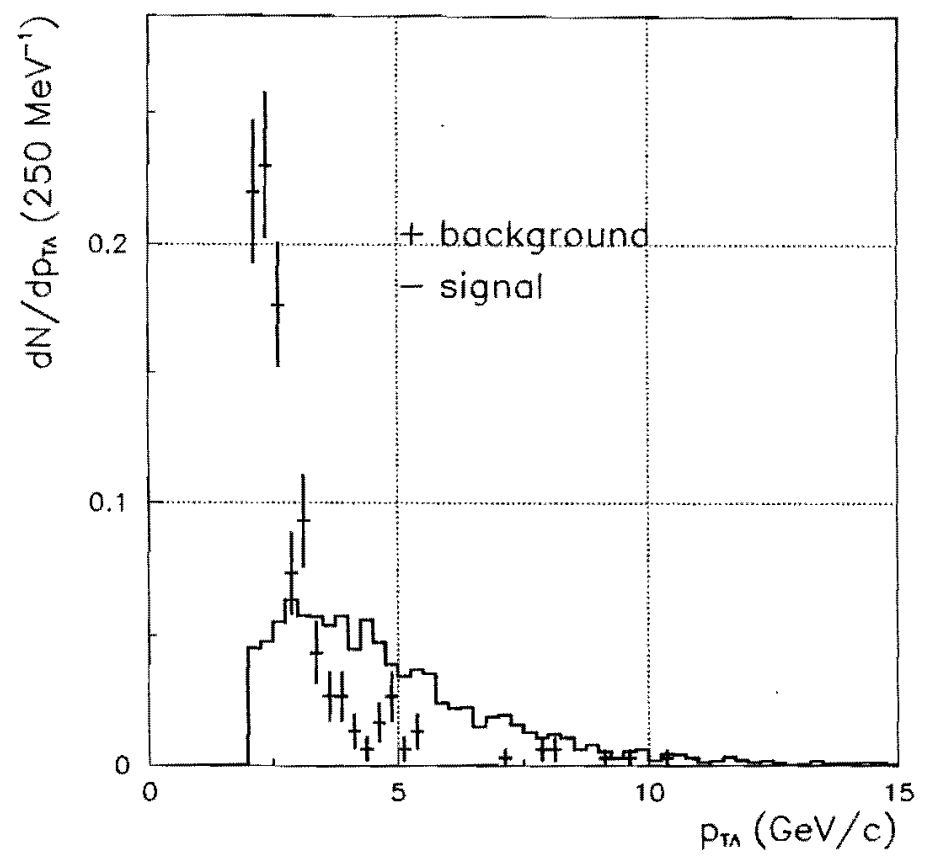

Figure 3.22: $p_{T \Lambda}$ distributions for the CTC $\Lambda_{b}$ side band events and the signal $\mathrm{MC}$.

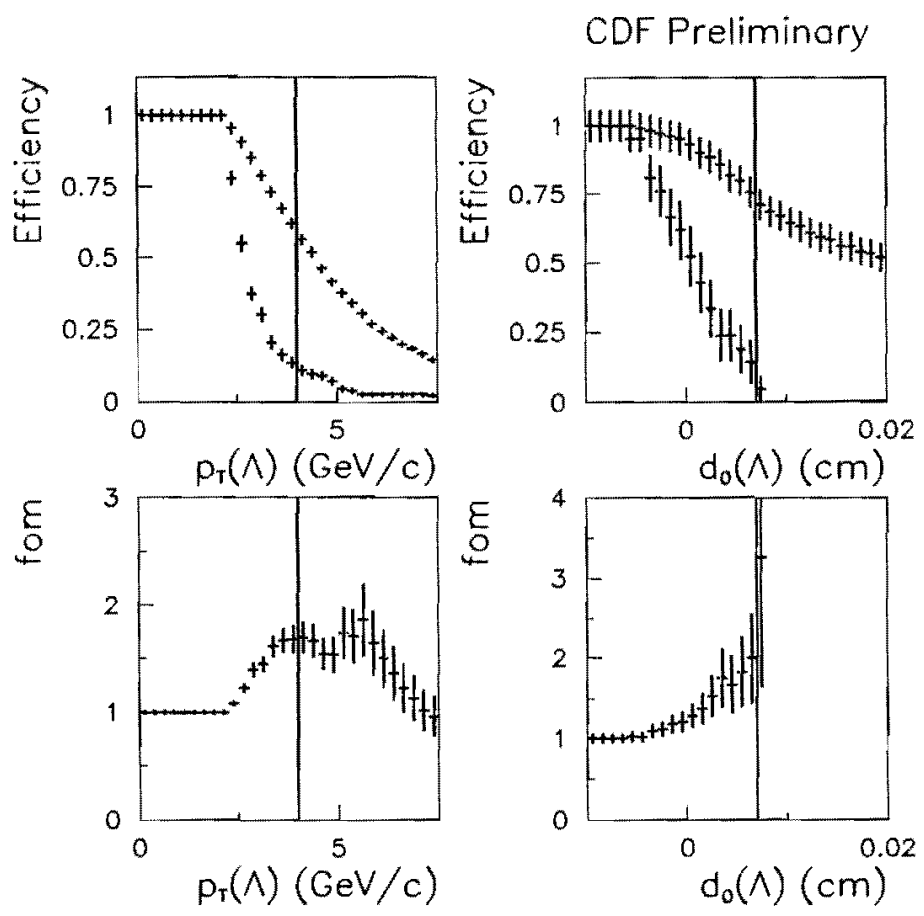

Figure 3.23: $\epsilon_{s i g}, \epsilon_{b g}$, and fom distribution as a function of the $p_{T \Lambda}\left(d_{0 \Lambda}\right)$ for the cut optimization of the CTC (SVX) $\Lambda_{b}$. 


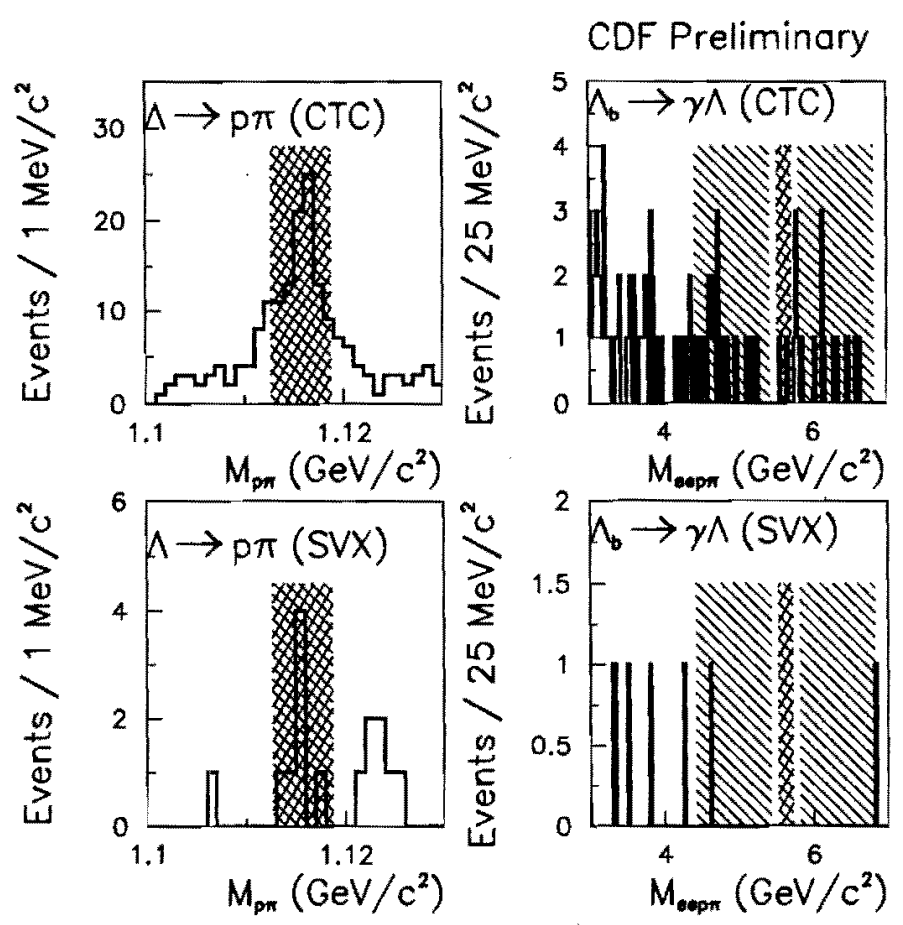

Figure 3.24: $M_{\Lambda}$ (left) and $M_{\Lambda_{b}}$ (right) distributions for $\Lambda_{b} \rightarrow \Lambda \gamma$ candidates after the optimized selection with $\Lambda$ reconstructed using the CTC tracks (top) and SVX tracks (bottom). 


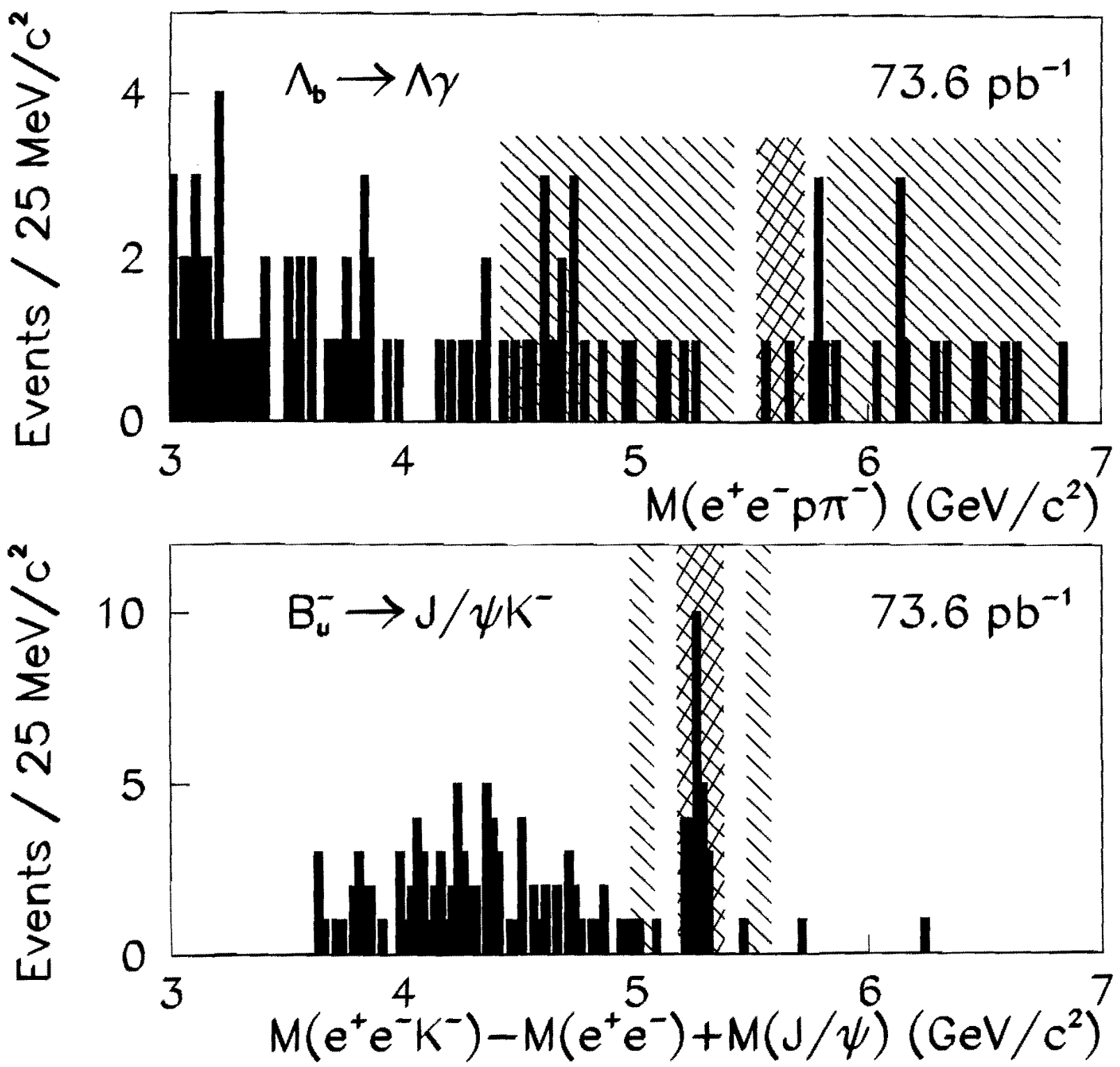

Figure 3.25: $\Lambda_{b} \rightarrow \Lambda \gamma$ and $\bar{B}_{u} \rightarrow J / \psi K^{-}$candidates after the optimized selection. 


\section{Chapter 4}

\section{Estimating the Efficiencies}

In this chapter, we discuss the estimation of the efficiency for the radiative $B$ decays which is necessary to set the limit of the branching fraction. The efficiency was estimated in terms of the ration between the radiative $B$ decays and the $\bar{B}_{u} \rightarrow J / \psi \mathrm{K}^{-}$decay.

\subsection{Efficiencies Classification}

The numbers of the radiative $B$ decay signal candidates and the $\bar{B}_{u} \rightarrow J / \psi K^{-}$reference candidates are given by

$$
\begin{aligned}
N_{\bar{K}^{* 0} \gamma} & =2 \cdot \sigma_{b \bar{b}} \cdot f_{d} \cdot B\left(\bar{B}_{d} \rightarrow \bar{K}^{* 0} \gamma\right) \cdot B\left(\bar{K}^{* 0} \rightarrow K^{-} \pi^{+}\right) \cdot \epsilon\left(K^{*} \gamma\right) \cdot \mathcal{L}, \\
N_{\phi \gamma} & =2 \cdot \sigma_{b \bar{b}} \cdot f_{s} \cdot B\left(\bar{B}_{s} \rightarrow \phi \gamma\right) \cdot B\left(\phi \rightarrow K^{+} K^{-}\right) \cdot \epsilon(\phi \gamma) \cdot \mathcal{L}, \\
N_{\Lambda \gamma} & =2 \cdot \sigma_{b \bar{b}} \cdot f_{\Lambda_{b}} \cdot B\left(\Lambda_{b} \rightarrow \Lambda \gamma\right) \cdot B\left(\Lambda \rightarrow p \pi^{-}\right) \cdot \epsilon(\Lambda \gamma) \cdot \mathcal{L}, \\
N_{J / \psi K} & =2 \cdot \sigma_{b \bar{b}} \cdot f_{u} \cdot B\left(\bar{B}_{u} \rightarrow J / \psi K^{-}\right) \cdot B(J / \psi \rightarrow e e) \cdot \epsilon(J / \psi K) \cdot \mathcal{L},
\end{aligned}
$$

where $\sigma_{b \bar{b}}$ is the $b \bar{b}$ production cross section, $f_{u}, f_{d}, f_{s}$, and $f_{\Lambda_{b}}$ are the fragmentation fraction of the $b$ quark to $\bar{B}_{u}, \bar{B}_{d}, \bar{B}_{s}$, and $\Lambda_{b}$ hadrons, respectively, $B(a \rightarrow b)$ is branching fractions for each channel, $\epsilon$ is the detection efficiency in our detector for each decay channel, and $\mathcal{L}$ is the integrated luminosity of the data set.

By using the observed $\bar{B}_{u} \rightarrow J / \psi K^{-}$candidates for the normalization of the radiative $B$ decays, the $b$-quark production cross section and the integrated luminosity of the data set cancel out. The fragmentation fractions, branching fractions, and efficiencies do not cancel in principle. Normalizing to the $\bar{B}_{u} \rightarrow J / \psi K^{-}$decays, the number of the radiative decays are given by,

$$
\begin{aligned}
& \frac{N_{K^{*} \gamma}}{N_{J / \psi K}}=\frac{f_{d}}{f_{u}} \times \frac{B\left(\bar{B}_{d} \rightarrow \bar{K}^{* 0} \gamma\right) B\left(\bar{K}^{* 0} \rightarrow K^{-} \pi^{+}\right)}{B\left(\bar{B}_{u} \rightarrow J / \psi K^{-}\right) B(J / \psi \rightarrow e e)} \times \frac{\epsilon\left(K^{*} \gamma\right)}{\epsilon(J / \psi K)} \\
& \frac{N_{\phi \gamma}}{N_{J / \psi K}}=\frac{f_{s}}{f_{u}} \times \frac{B\left(\bar{B}_{s} \rightarrow \phi \gamma\right) B\left(\phi \rightarrow K^{+} K^{-}\right)}{B\left(\bar{B}_{u} \rightarrow J / \psi K^{-}\right) B(J / \psi \rightarrow e e)} \times \frac{\epsilon(\phi \gamma)}{\epsilon(J / \psi K)}
\end{aligned}
$$




$$
\frac{N_{\Lambda \gamma}}{N_{J / \psi K}}=\frac{f_{\Lambda_{b}}}{f_{u}} \times \frac{B\left(\Lambda_{b} \rightarrow \Lambda \gamma\right) B(\Lambda \rightarrow p \pi)}{B\left(\bar{B}_{u} \rightarrow J / \psi K^{-}\right) B(J / \psi \rightarrow e e)} \times \frac{\epsilon(\Lambda \gamma)}{\epsilon(J / \psi K)}
$$

We use the ratios of the $b$-quark fragmentation fractions of $f_{d} / f_{u}=1, f_{s} / f_{u}=0.426$ \pm 0.076 , and $f_{\Lambda_{b}} / f_{u}=0.236 \pm 0.082 \quad[40]$, and the branching fraction of $(0.99 \pm 0.10)$ $\times 10^{-3},(6.02 \pm 0.19) \%, 2 / 3,0.491 \pm 0.06$, and $0.639 \pm 0.005$ for $B\left(\bar{B}_{u} \rightarrow J / \psi K^{-}\right)$, $B(J / \psi \rightarrow e e), B\left(\bar{K}^{* 0} \rightarrow K^{-} \pi^{+}\right), B\left(\phi \rightarrow K^{+} K^{-}\right)$, and $B\left(\Lambda \rightarrow p \pi^{-}\right)$, respectively [6]. The detection efficiencies for each channel are given by

$$
\begin{aligned}
\epsilon\left(K^{*} \gamma\right) & =p_{c o n v} \times A \times \epsilon_{t r g} \times \epsilon_{C T C} \times \epsilon_{S V X} \times \epsilon_{e l e} \times \epsilon_{f i t} \times \epsilon_{i s o}, \\
\epsilon(\phi \gamma) & =p_{c o n v} \times A \times \epsilon_{t r g} \times \epsilon_{C T C} \times \epsilon_{S V X} \times \epsilon_{e l e} \times \epsilon_{f i t} \times \epsilon_{i s o}, \\
\epsilon(\Lambda \gamma) & =p_{c o n v} \times A \times \epsilon_{t r g} \times \epsilon_{C T C} \times \epsilon_{S V X} \times \epsilon_{e l e} \times \epsilon_{\Lambda} \times \epsilon_{f i t} \times \epsilon_{i s o}, \\
\epsilon(J / \psi K) & =A \times \epsilon_{t r g} \times \epsilon_{C T C} \times \epsilon_{S V X} \times \epsilon_{e l e} \times \epsilon_{s e l} \times \epsilon_{f i t} \times \epsilon_{i s o},
\end{aligned}
$$

where,

$p_{\text {conv }}$ : conversion probability,

$\epsilon_{\text {trg }}$ : trigger efficiency,

$A \quad$ : acceptance (fiducial, $E_{T}, p_{T}$, mass, $c \tau^{*}, d_{0} / \sigma$ ),

$\epsilon_{C T C}$ : CTC track reconstruction and quality cut efficiency,

$\epsilon_{S V X}:$ SVX track reconstruction and quality cut efficiency,

$\epsilon_{\text {ele }} \quad$ : trigger electron quality cut efficiency,

$\epsilon_{s e l}:$ soft electron quality cut efficiency,

$\epsilon_{\Lambda} \quad: \Lambda \rightarrow p \pi$ quality cut efficiency,

$\epsilon_{f i t}:$ vertex fit quality cut efficiency,

$\epsilon_{i s o}: B$ isolation cut efficiency.

To be more specific and as a guide for what we envisage in the estimation of the efficiency ratios, we categorize efficiencies into three classes according to the extent of cancelation in the ratios:

For the estimation of the efficiency ratios $\left(\frac{\epsilon\left(K^{*} \gamma\right)}{\epsilon(J / \psi K)}, \frac{\epsilon(\phi \gamma)}{\epsilon(J / \psi K)}\right.$, and $\left.\frac{\epsilon(\Lambda \gamma)}{\epsilon(J / \psi K)}\right)$, we categorize efficiencies into three classes according to the extent of the cancelation in the efficiency ratios: (1) efficiencies particular to a specific decay mode, thus they and thier uncertainties directly come in the ratios, without canceling. We estimate these efficiencies by relying as much as possible on data; (2) efficiencies similar between the radiative and the reference decay for which they are expected to be nearly equal, and the uncertainties have small impact on the ratios. Thus we concern about small correction factors with respect to unity for the efficiency ratios and rely on Monte Carlo calculations for the most part; (3) efficiencies assumed to be common between the radiative and the reference decay, thus exactly cancel in the ratio of the branching fractions. We discuss each 
class and treatment of in the following sections Note that the tracking and vertexing efficiency spread over several classes and we discuss them separately.

\subsection{Class 1 Efficiencies}

The conversion probability $\left(p_{c o n v}\right)$, the efficiencies for the $J / \psi$ partner electron quality cuts $\left(\epsilon_{s e l}\right)$, and the quality cut for $\Lambda\left(\epsilon_{\Lambda}\right)$ fall under class 1 .

\subsubsection{Conversion Probability}

Since the photon conversion probability $(6 \%)$ is mainly responsible for the the efficiency ratio between the radiative and reference channels beeing less than 1 , we should treat it carefully. The Attenuation length of the photon with $p>1 \mathrm{GeV} / c$ in a material due to the photon conversion is assumed to be $9 / 7 X_{0}$, where $X_{0}$ is radiation length of the material. Thus the photon conversion probability passing through the material with thickness of $X$ is given by

$$
p_{\text {conv }}=1-e^{-\frac{7}{9} \frac{X}{X_{0}}} \sim \frac{7}{9} \frac{X}{X_{0}},
$$

In our detector, the $p_{c o n v}$ is described as

$$
p_{\text {conv }} \sim \frac{7}{9} \int \frac{d s}{X_{0}(\vec{r})}=\frac{7}{9} \int \frac{d r}{X_{0}(\vec{r})|\sin \theta|},
$$

where $X_{0}(\vec{r})$ is the radiation length of the material located at the position $\vec{r}=(x, y, z)$, and $d s$ and $d r$ represents the track of the photon and its projection to the $x y$ plane, respectively. Since the photon track is a straight line the $\sin \theta$ is a constant. we can assume the probability does not depends on the azimuthal angle of the photon, since the CDF detector is designed with an overall cylindrical symmetry, Then we can write as,

$$
p_{c o n v}=\frac{7}{9} \frac{1}{\sin |\theta|} \int \frac{d r}{X_{0}(r, z)} \equiv \frac{7}{9} \frac{X_{r}\left(z_{0}, \theta\right)}{|\sin \theta|}
$$

The integrated material thickness in the transverse plane $\left(X_{T}\right)$ depends on the $z_{0}$ and $\theta$ of the photon. For example, the SVX whose material thickness corresponds to $X_{T}$ $\sim 2.5 \% X_{0}$ only covers the region $|z|<30 \mathrm{~cm}$. Thus most of the photons produced with $\left|z_{0}\right|>30 \mathrm{~cm}$ are expected to pass through $2.5 \%$ less materials than that inside the 
SVX fiducial. The $\theta$ dependence of the $X_{T}$ is expected to be much smaller than the $z_{0}$ dependence, but it appears in such a way that, for example, the photon with $\left|z_{0}\right|<30$ cm but with larger $|\eta|$ can exit the SVX fiducial earlier.

Then, the conversion probability averaged over a particular radiative $B$ decay channel is given by

$$
<p_{\text {conv }}>=\frac{1}{N} \sum_{i=1}^{N} \frac{7}{9} \frac{X_{T}\left(z_{0 i}, \theta_{i}\right)}{\left|\sin \theta_{i}\right|} \equiv \frac{7}{9}<X_{T}><|\sin \theta|^{-1}>
$$

The MC detector simulation handles the photon conversion process. This uses the material distribution of the CDF inner detector based on measurements by using CDF data, and the conversion length is assumed to be (9/7) $X_{0}$ for photons with $p>1 \mathrm{GeV} / c$. At first, the simulation determines the photon decay length according to a probability function of $e^{-\frac{7}{8} \frac{X}{x_{0}}}$, then accumulate the thickness of the materials, which the photon is passing through step by step (beam pipe, SVX L1, L2, L3, L4, etc., up to the CTC inner wall). If the sum of the material thickness exceeds the decay length, the photon conversion occurs at the step. Otherwise the photon goes to the calorimeter.

The resulting conversion probability from the MC calculations is $\sim 6 \%$. Figures 4.1 and 4.2 show the $x y$-view and $r z$-view cross section, respectively, of the conversion vertex distribution obtained from the MC sample. These MC distributions reproduce the data distributions (Figures 2.11 and 2.12) at a $10 \%$ level. Note that our detector simulation only simulates the conversion before the CTC inner wall

We further calibrate the MC material thickness by using the photon conversion candidates in our data. The CTC inner wall has a uniform structure in the $z$ and $\phi$ directions, and its transverse thickness is well understood to be $X_{T}(\mathrm{CTC})=(1.26 \pm$ $0.06) \% X_{0}$. Then, the average transverse thickness of the material $\left(\left\langle X_{T}\right\rangle\right)$ is obtained by scaling $X_{T}(\mathrm{CTC})$ by the ratio between the total number of photon conversions and the number of photon conversions that occur at the CTC inner wall:

$$
<X_{T}>=X_{T}(\mathrm{CTC}) \times \frac{N(\text { all })}{N(\mathrm{CTC})},
$$

where $N(\mathrm{CTCn})$ is the number of conversions in the CTC inner wall, and $N($ all $)$ is the number of conversions before the CTC tracking volume.

In order to ensure the correct photon geometry relevant to our analysis for the $\bar{B}_{d}$ and $\bar{B}_{s}$ decays, we use $\bar{B}_{d} \rightarrow \bar{K}^{* 0} \gamma$ candidates (conversion +2 SVX track sample), but with loose selection cuts on $c \tau^{*}, I_{B}$, and mass windows to gain statistics. For the $\Lambda_{b}$ case on the other hand, we simply use the photon conversion candidates without any 


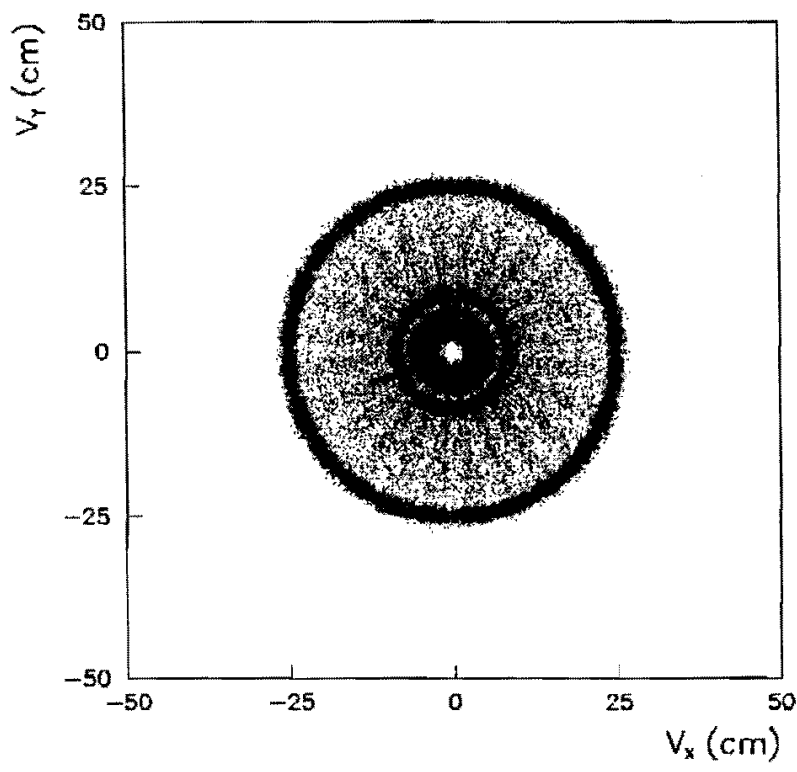

Figure 4.1: A $x y$-view of the photon conversion vertex distribution in the $\mathrm{MC}$ sample.

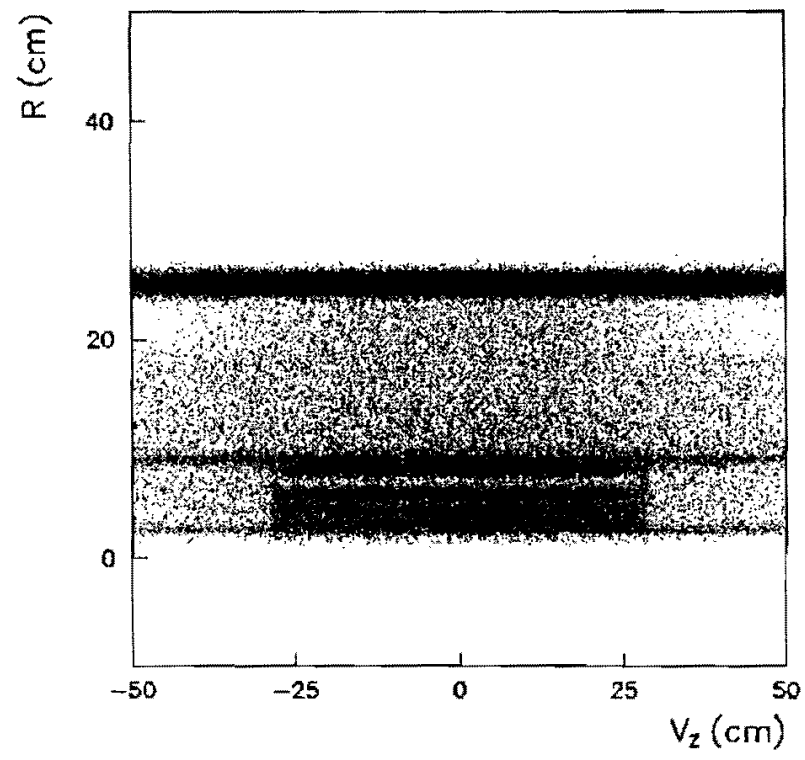

Figure 4.2: A $r z$-view of the photon conversion vertex distribution in the MC sample. 
requirements on other tracks, which yield $\sim 0.4 \% X_{0}$ less material compared to the $B$ meson case.

We do the same analysis for both the data and the $\mathrm{MC}$, then take the ratio $X_{T}(\mathrm{DATA}) / X_{T}(\mathrm{MC})$ as the correction factor for the conversion probability. These factors are found to be $0.89 \pm 0.05$ for the $\bar{B}_{d}$ and $\bar{B}_{s}$ decays, and $0.95 \pm 0.05$ for the $\Lambda_{b}$ decay. Details of the estimation are shown in Appendix A.

\subsubsection{Soft Electron Quality Cut Efficiency for $J / \psi \rightarrow e e$}

The soft electron quality cuts fonsist of two quality cuts for the partner electron, namely the $d E / d x$ cut and the $E / p$ cut.

Since the $d E / d x$ is defined as the $\Delta / \sigma$ (See Equation 2.2), the $d E / d x>-1.27$ cut efficiency is $90 \%$ by definition. We check the efficiency for the $d E / d x>-1.27$ by using the partner electrons of the conversion candidates which form a very high purity electron sample. We estimate the efficiency by just counting the number of the conversion candidate before and after the $d E / d x$ cut. Figure 4.3 shows the $d E / d x$ distribution of the conversion electrons (top plot) and the $d E / d x>-1.27$ cut efficiency as a function of the track $p_{T}$ (bottom plot). In these plots, we require the partner electron to be in the different wedge of the trigger electron The efficiency shows no $p_{T}$ dependence and the averaged efficiency is 0.90 and its uncertainty is negligible. Actually the $d E / d x$ was calibrated by using the conversion sample, so the result is obvious.

The $E / p>0.5$ cut efficiency is estimated by using the $J / \psi \rightarrow e e$ candidates themselves. Figure 4.4 shows the $J / \psi \rightarrow e e$ mass peak before and after the $E / p$ cut (top plot). We fit the distribution to a function of the Gaussian + Polynomial and estimate the averaged efficiency to be $0.83 \pm 0.03$. The efficiency shows no $p_{T}$ dependence (bottom plot).

Combining the $d E / d x$ and $e / p$ cuts, we estimate the quality cut efficiency for the $J / \psi \rightarrow$ ee partner electron to be $0.90 \times(0.83 \pm 0.03)=0.75 \pm 0.03$.

\subsubsection{Quality Cut Efficiency for $\Lambda \rightarrow p \pi$}

We apply three quality cuts for the $\Lambda$, namely the $d E / d x$ cut, the conversion removal, and the impact parameter cut.

The efficiency for the $d E / d x$ cut and the conversion removal is estimated by using the $\Lambda \rightarrow p \pi$ candidates themselves. We use a $\gamma+$ CTC $\Lambda$ sample with a loose selection 

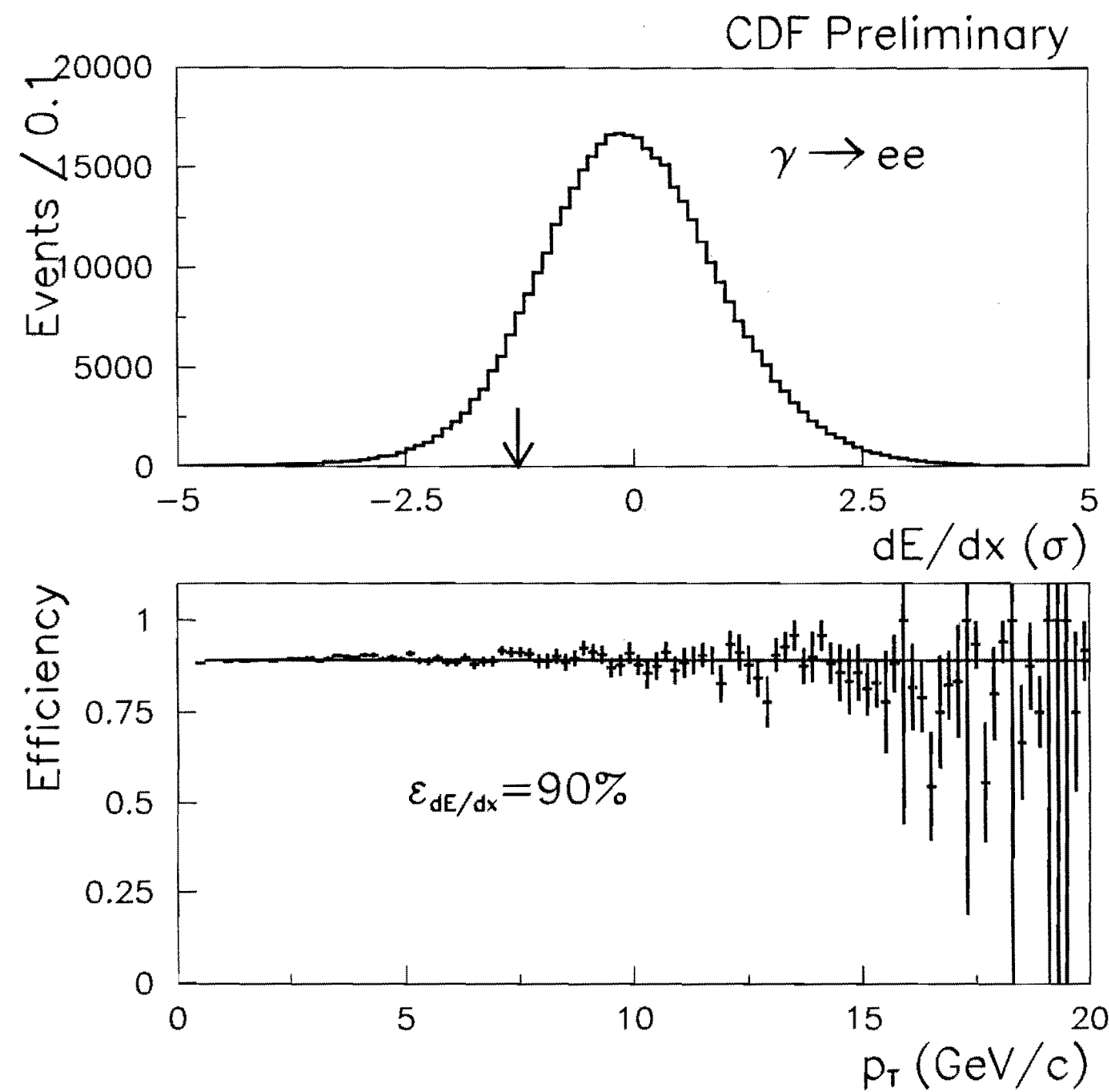

Figure 4.3: $d E / d x$ distribution for the partner track of the trigger conversion candidates (top), and the efficiency of $d E / d x>-1.27$ as a function of track $p_{T}$ (bottom). 

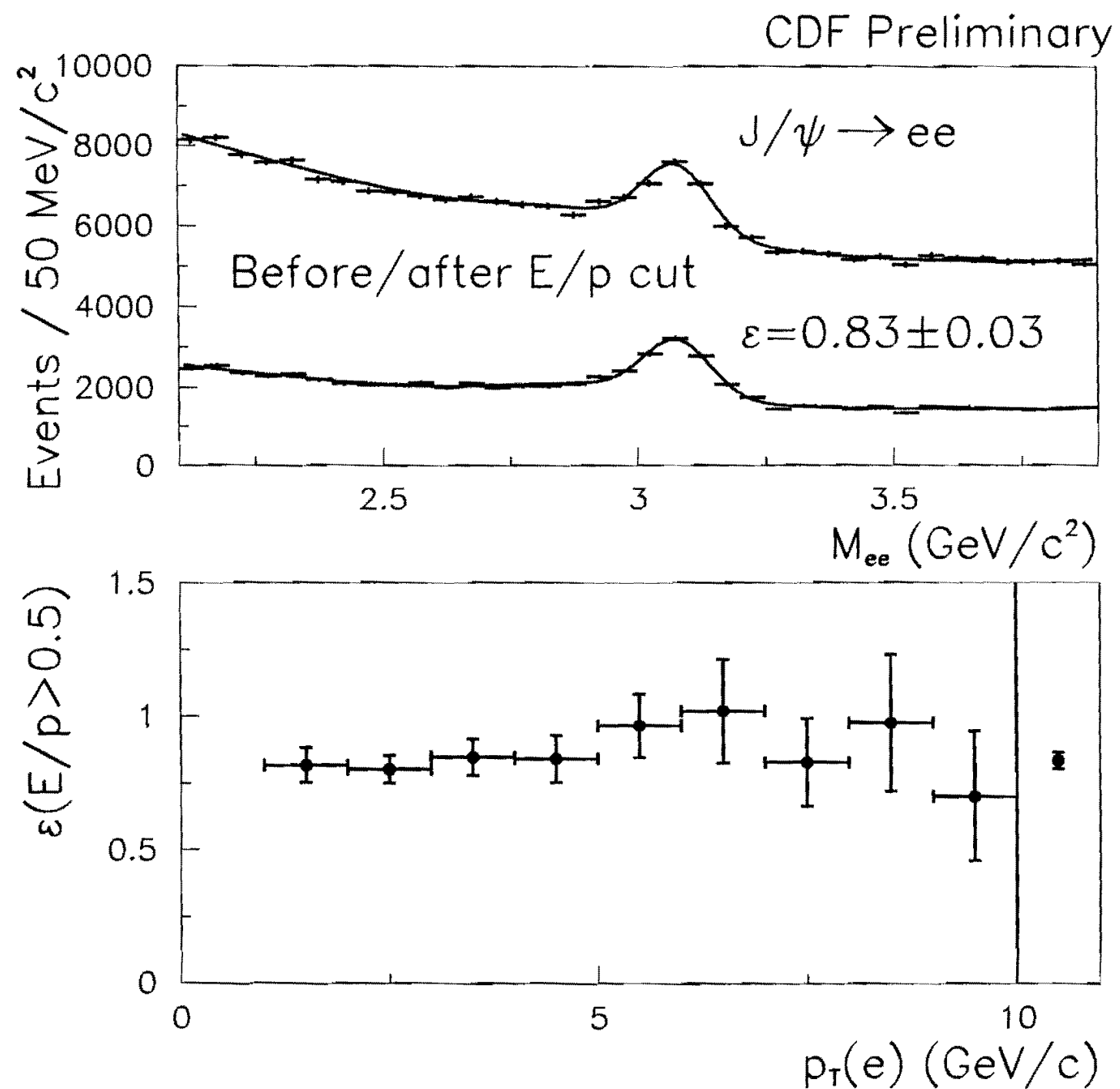

Figure 4.4: $M_{e e}$ distribution for the trigger $J / \psi \rightarrow e e$ candidates before and after $E / p>0.5$ cut for the partner track (top), and the cut efficiency as a function of track $p_{T}$ (bottom). 

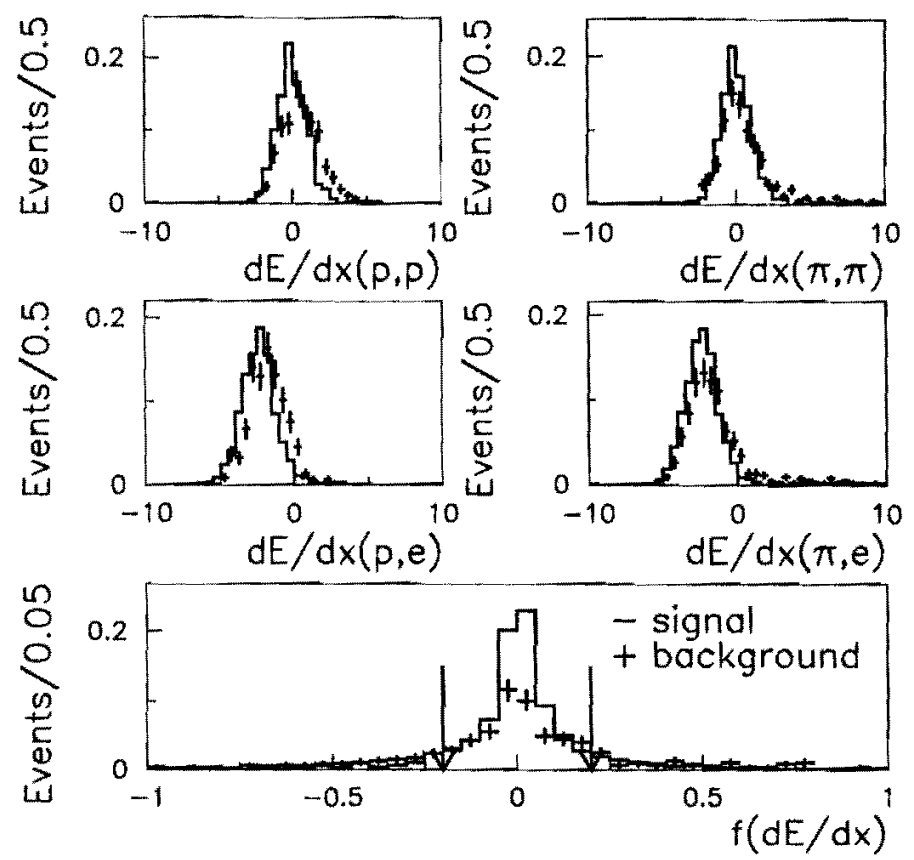

Figure 4.5: The $d E / d x$ distributions for the $\Lambda \rightarrow p \pi$ signal (histograms), and the ackground estimated from the $\Lambda$ mass side band events (points).

requiring no $B$ isolation and wide $B$ mass window. Figure 4.5 shows several $d E / d x$ and $f(d E / d x)$ distributions. The distribution for the events in the $\Lambda$ mass signal region (histograms) and $\Lambda$ mass side band events (points) are shown. Figure 4.6 shows the $\Lambda \rightarrow p \pi$ mass peak before and after the $|f(d E / d x)|<0.2$ and conversion removal cut (top plot). We fit the mass distribution to a function of the Gaussian + straight line, and estimate the efficiency to be $0.721 \pm 0.018$. The efficiency shows no $p_{T}$ dependence (bottom plot).

The large impact parameter for the proton and pion from the $\Lambda$ is not caused by the displacement of the $\Lambda_{b}$ decay vertex but the $\Lambda$ decay vertex. The directions of these tracks are almost the same as that of the $\Lambda$, and the impact parameter is rather caused by the bending of the tracks in the solenoidal magnetic field. We estimate the efficiency for the impact parameter cut, $\left|d_{0} / \sigma(p)\right|>0.5$ and $\left|d_{0} / \sigma(\pi)\right|>2.0$, by relying on the $\mathrm{MC}$, and check them by using the data. Figure 4.7 shows the $d_{0} / \sigma$ distributions for the proton and pion in the data (histograms) and MC (points). The cut efficiencies as a function of the $p_{T}(\Lambda)$ are shown in Figure 4.8. The obtained efficiencies are $(78.6 \pm 1.1) \%$ for data (top) an ( $82.0 \pm 1.1) \%$ for $\mathrm{MC}$ (bottom). We see no significant $p_{T}(\Lambda)$ dependence in both the data and MC. The difference between the data and MC is small and we use the $\mathrm{MC}$ efficiency in the following analysis. 


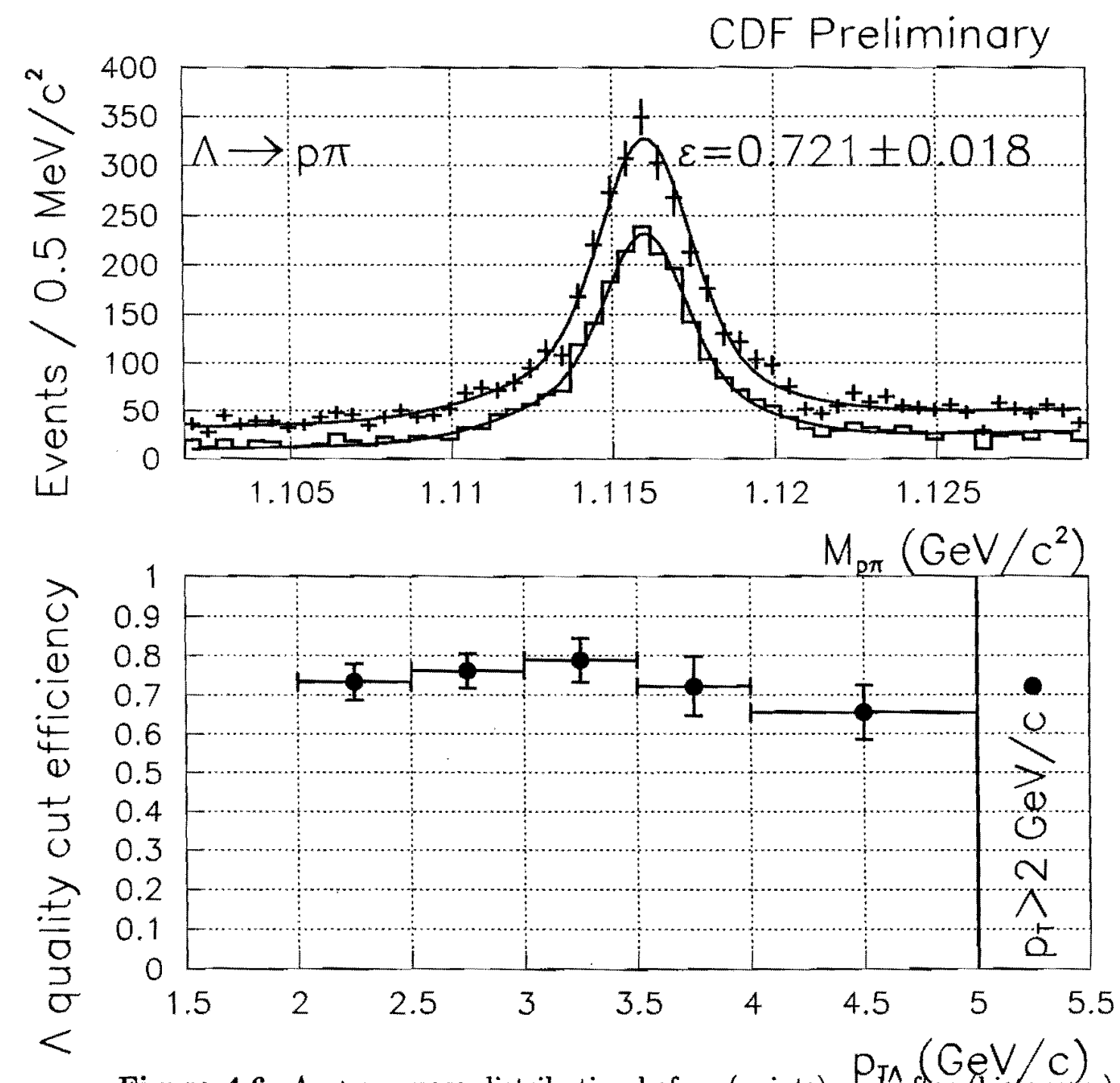

Figure 4.6: $\Lambda \rightarrow p \pi$ mass distribution before (points) a fis after (histogram) the $d E / d x$ and conversion removal cuts (top), and the efficiency as a function of $p_{T \Lambda}$ (bottom). 

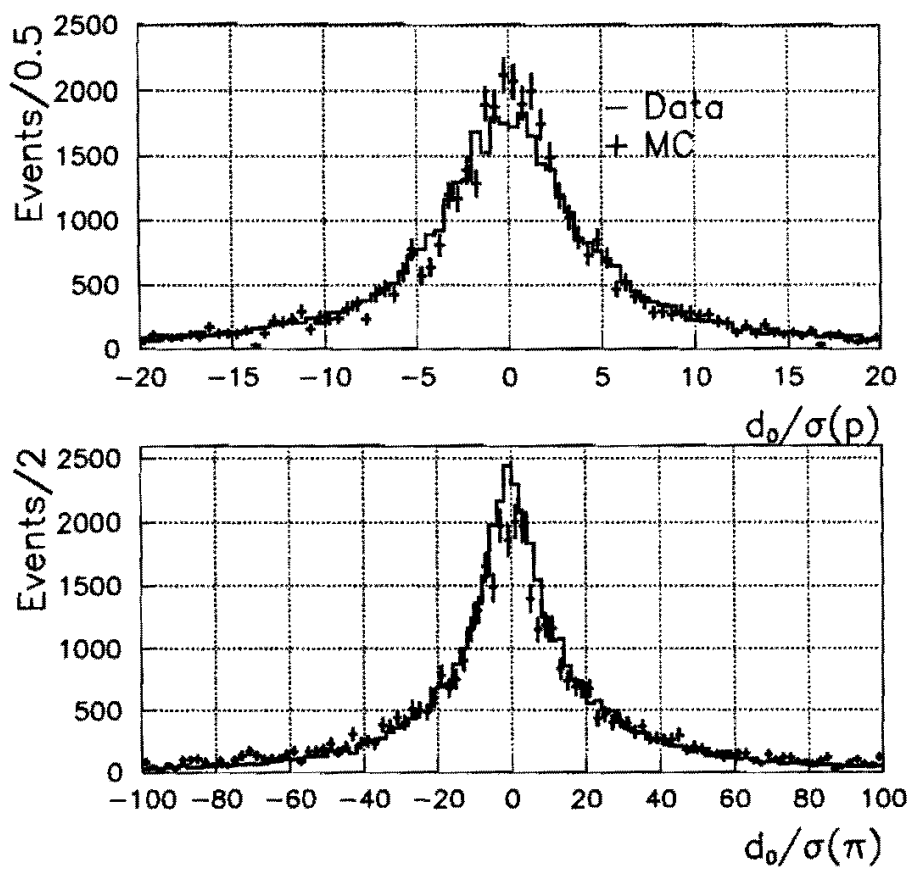

Figure 4.7: Impact parameter significance distributions for the proton and pion from the $\Lambda$.
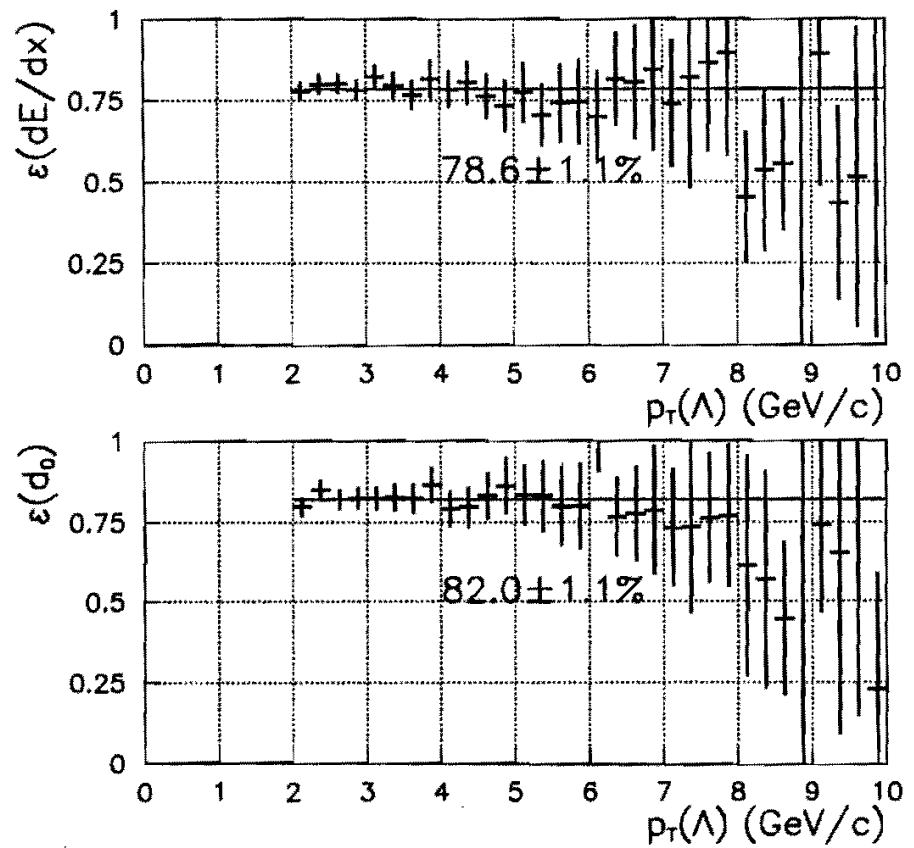

Figure 4.8: $d_{0} / \sigma$ cut efficiency for $\Lambda \rightarrow p \pi$ as a function of $p_{T \Lambda}$ for the data (top) and the MC (bottom). 


\subsection{Class 2 Efficiencies}

Various kinematical and geometrical acceptances $(A)$ which include the $E_{T}, p_{T}$, mass window, $c \tau^{*}$, and impact parameter cuts, and efficiencies for the trigger $\left(\epsilon_{t r g}\right)$, trigger electron quality cuts $\left(\epsilon_{e l e}\right)$, and fitting constraints $\left(\epsilon_{f i t}\right)$ fall under class 2 . Efficiencies for most of such requirements are similar between the radiative decays and the reference channel and their ratios are close to 1 , however, we still need to estimate small correction factors for the efficiency ratios. For the estimation of these efficiency ratios we rely on MC calculations. Here we discuss details about the level 2 trigger efficiency and electron quality cut efficiency.

\subsubsection{Level 2 Trigger Efficiency}

The Level 2 trigger efficiency is estimated by applying an efficiency turn-on curve as a function of electron $E_{T}$ and $p_{T}$ to the MC sample. The trigger efficiency curve is measured by using non-biased CDF data collected with independent triggers.

The trigger efficiencies for the CEM $(8 \mathrm{GeV})$ and CFT $(7.5 \mathrm{GeV} / c)$ requirements are estimated by using a trigger sample with the lower CEM (5 GeV) and CFT (4.7 $\mathrm{GeV} / \mathrm{c}$ ) requirements. Whatever we look at the CEM and CFT efficiencies as a function of the offline $E_{T}$ and $p_{T}$, respectively, the efficiency curves basically do not depend on whether the sample is the real electron or the hadron fake.

If we look at the XCES trigger efficiency as a function of the offline CES cluster energy, it is the same between the electron and the hadron fake. However, if we look at the efficiency as a function of the $E_{T}$, the efficiency curve can differ between the electron and the hadron fake. Thus we need a high purity electron sample to do the estimation and use the conversion candidates.

The efficiency curve is obtained to be,

$$
\begin{aligned}
& \epsilon_{L 2}=\quad 0.914 \quad \text { :Asymptotic eff. including L1 } \\
& \times \quad 0.992 \quad: H a d / E M \\
& \times \quad 1.0 \pm 0.044 \quad \text { :Error for the asymptotic efficiency } \\
& \times \quad \text { Freq }\left(\frac{E_{T}-2.90}{1.42 \times \sqrt{E_{T}}}\right) \quad \text { :Tower boundary correction } \\
& \times \text { Freq }\left(\frac{p_{T}-5.900 \pm 0.014}{0.1895 \pm 0.0039 \times p_{T}}\right) \quad: \text { CFT_7_5 } \\
& \times \operatorname{Freq}\left(\frac{E_{T}-8.736 \pm 0.011}{1.660 \pm 0.0042 \times \sqrt{E_{T}}}\right) \quad: \text { CEM_8 } \\
& \times \operatorname{Freq}\left(\frac{E_{T}-5.31 \pm 0.16}{1.119 \pm 0.048 \times \sqrt{E_{T}}}\right) \quad: \mathrm{XCES} \text {, }
\end{aligned}
$$




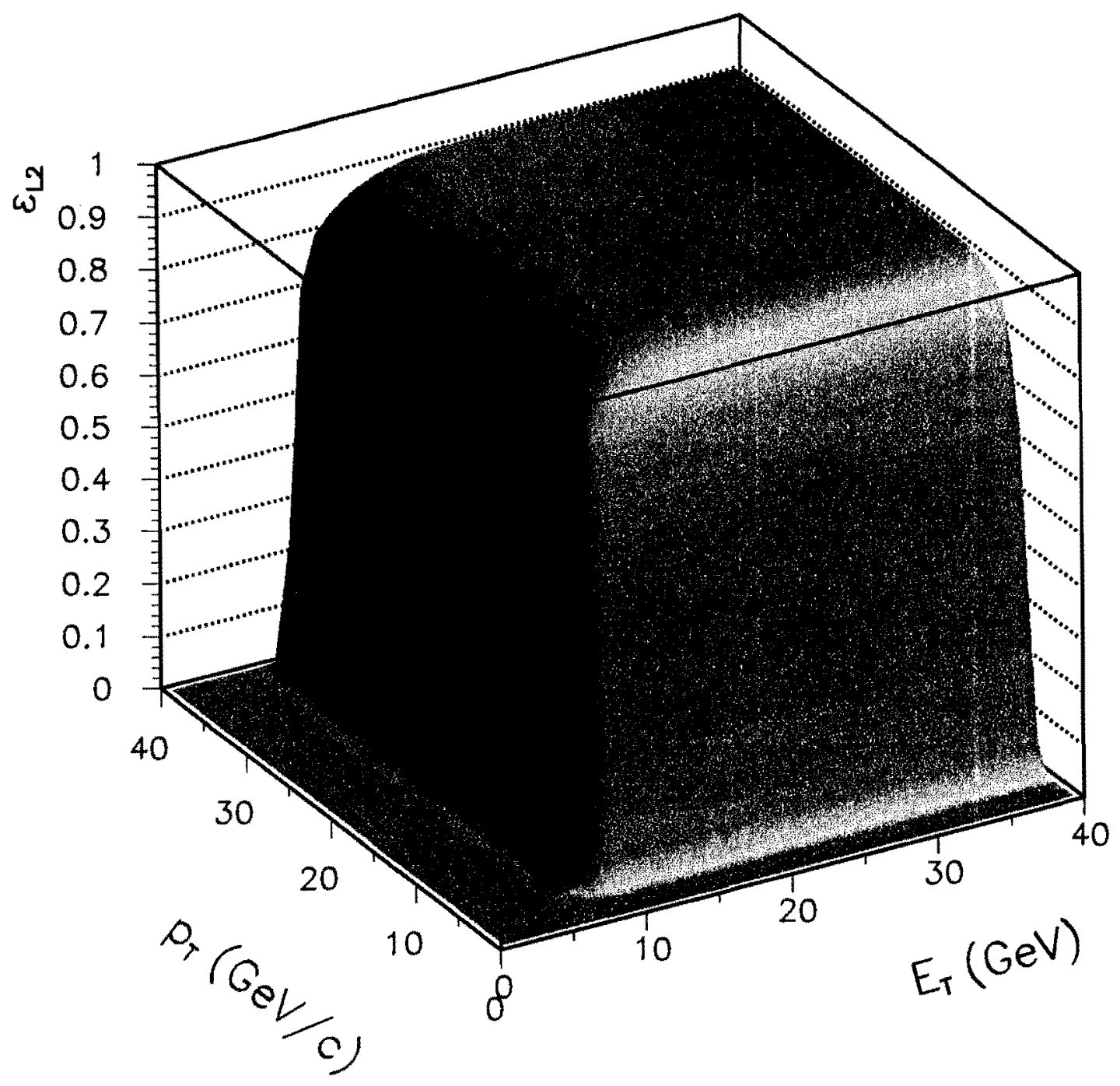

Figure 4.9: L2 CEM_8_CFT_7_5_XCES trigger efficiency as a function of $E_{T}$ and $p_{T}$.

where Freq $(x)$ is defined by,

$$
\operatorname{Freq}(x)=\frac{1}{\sqrt{2 \pi}} \int_{-\infty}^{x} e^{-\frac{1}{2} t^{2}} d t
$$

\subsubsection{Trigger Electron Quality Cut Efficiency}

The trigger electron quality cut efficiency is handled by using the MC sample. We check its reliability by using partner electrons of the $J / \psi \rightarrow e e$ candidates.

Figure 4.10 shows quality cut parameter distributions for the partner electron of the $J / \psi \rightarrow e e$ candidates (points), and $J / \psi K$ signal MC (histograms). The background 

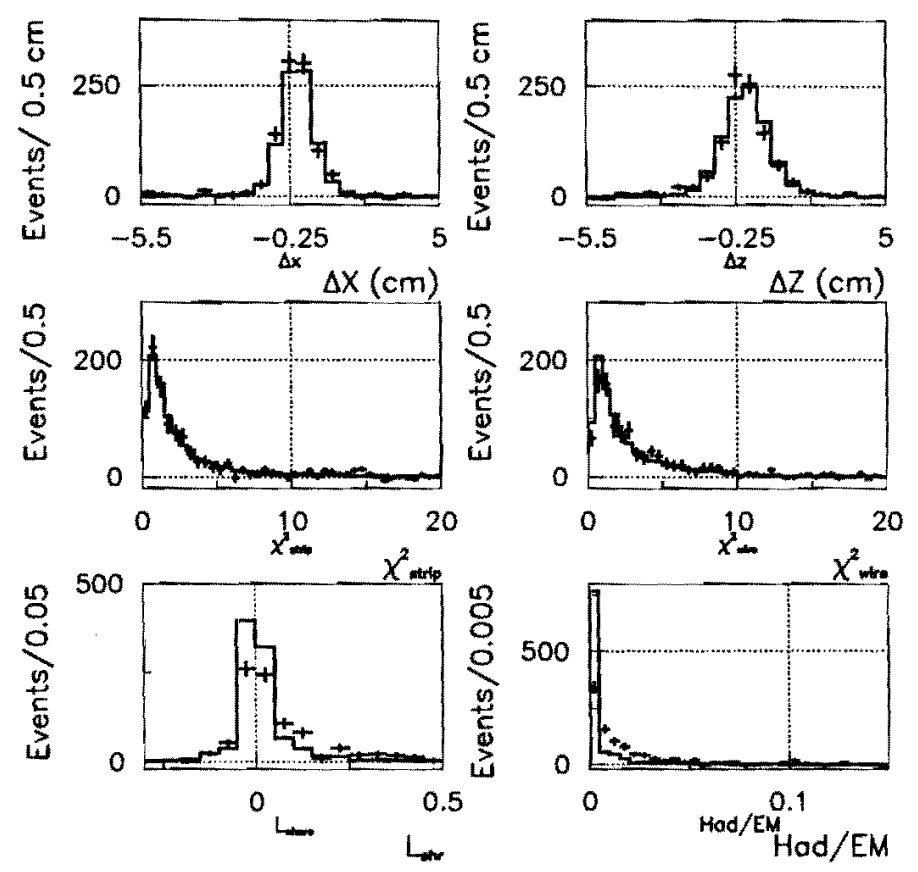

Figure 4.10: Distributions of the electron quality cut parameters for the partner electron of the $J / \psi \rightarrow e e$ candidates (points) and the $J / \psi K \mathrm{MC}$ sample (histograms).

of $J / \psi \rightarrow e e$ candidates is subtracted. The MC simulates $\Delta x, \Delta z, \chi_{s t r i p}^{2}$, and $\chi_{w i r e}^{2}$ very well. Disagreements in the $L_{\text {share }}$ and $\mathrm{Had} / E M$ distributions are due to the fact that the signal MC does not simulate underlying events. Since we rely on MC calculations of the relative efficiency for these parameters, we discuss more details.

\subsubsection{1 $\quad L_{\text {share }}$}

The $L_{\text {share }}$ is based on the lateral shower profile in terms of calorimeter towers along the $z$ direction, and affected by the underlying products. We assume that underlying events originating from the non- $b$ particles such as fragmentation, multiple collision, etc, are common to both modes. Thus the effects are canceled in the ratio between the signal and reference channels. However some of the underlying products come from the $B$-hadron decay daughters such as the $\gamma, J / \psi \rightarrow e e$ partner electrons. This effect of $B$ hadron decay daughters could be different between the radiative and reference decays. For example, since two electrons from the conversion is parallel at the conversion point, $z$ positions of the conversion electrons at the calorimeter are very close, then basically the partner electron does not change $L_{\text {share }}$ so much. Figure 4.11 shows efficiency curves for trigger electrons of $K^{*} \gamma \mathrm{MC}$ and $J / \psi K \mathrm{MC}$. We apply the final selection cut (except $L_{\text {share }}$ ) optimized for $K^{*} \gamma$. For $J / \psi K$, the fraction that we find partner electrons in 

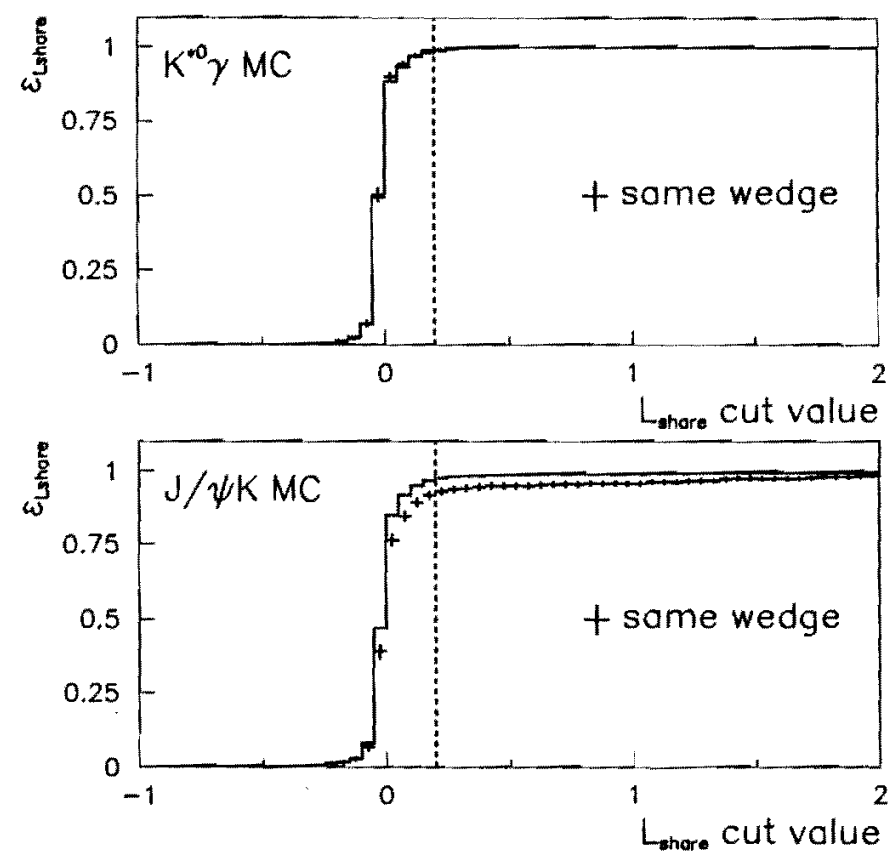

Figure 4.11: $L_{\text {share }}$ cut efficiency of the trigger electron of the $K^{*} \gamma$ and $J / \psi K$ $\mathrm{MC}$. The plot when the electrons are in a same wedge is also shown (points).

the same wedge as the trigger electrons is $22 \%$ and the efficiency is only $5 \%$ lower. For the $K^{*} \gamma$ channel, the fraction that we find partner electrons in the same wedge as the trigger electrons is $45 \%$, however, the efficiency does not change. It causes only $\sim 1 \%$ difference of the $L_{\text {share }}$ cut efficiency between the signal and background decays.

To check the effect of the non- $b$ underlying events, we look at the $J / \psi \rightarrow e e$ candidates in the data. Figure 4.12 shows efficiency curves for partner electrons of the $J / \psi \rightarrow e e$ candidates in data and that for the $J / \psi K$ MC sample is also shown. The case in which both electrons are in a same wedge is separately shown. The efficiency from the real data is $15 \%$ lower than the MC. This implies dominance of the non- $b$ underlying events over the effect from presence of another electron.

As a conclusion, the effect from partner electrons to $L_{\text {share }}$ efficiency is small and the MC predicts that the efficiencies for the radiative decays and $J / \psi$ decays are canceled in the relative efficiency. Underlying events change the efficiency by $15 \%$, but this does not hurt the efficiency cancelation, since underlying events are common to both decay modes. 

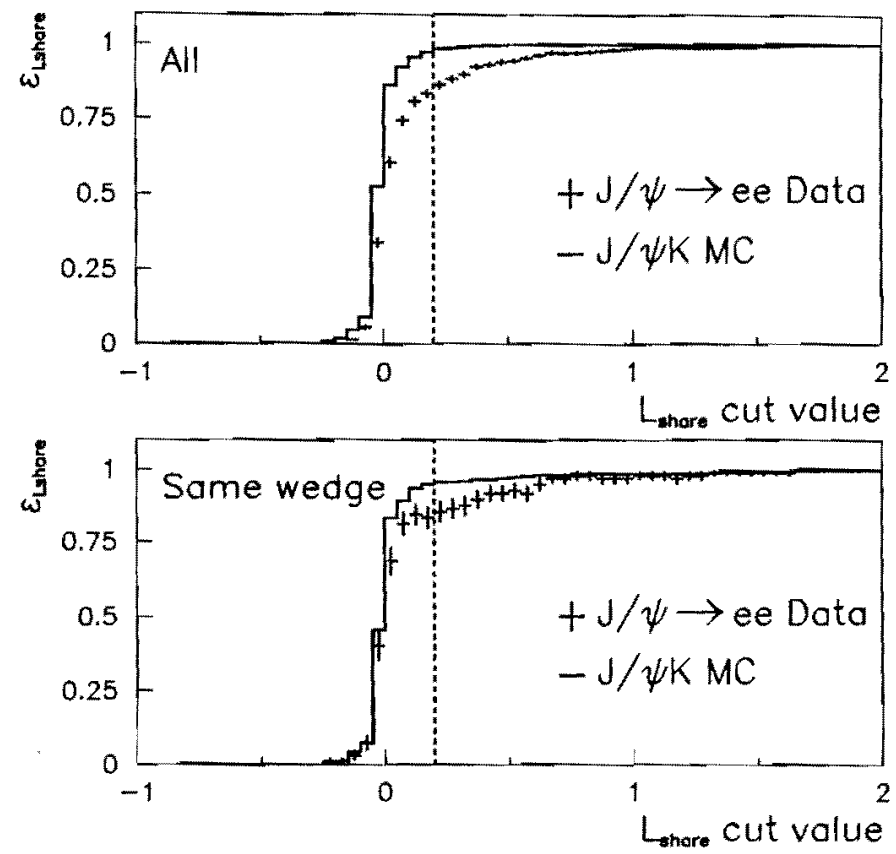

Figure 4.12: $L_{\text {share }}$ cut efficiency as a function of the cut value for the $J / \psi \rightarrow$ $e e$ candidates and $J / \psi K \mathrm{MC}$ sample. A plot when the electrons are in a same wedge is also shown (bottom).

\subsubsection{2 $\mathrm{Had} / \mathrm{EM}$}

The $\mathrm{Had} / E M$ is the ratio of the hadronic and electromagnetic energies sum over three calorimeter towers along the $z$ direction. Sources of the electromagnetic energy are the trigger electron for the most part, plus the partner electron and underlying events, while hadrons from $B$ decays and from underlying events contribute to the hadronic energy.

The effect of the hadrons from $B$ decays would be different between the radiative decays and the $J / \psi K$ decays because the $Q$ values are different and also the number of hadrons from the decays are different. Figure 4.13 shows $\mathrm{Had} / E M$ distributions of the trigger electrons for the $K^{*} \gamma, J / \psi K, J / \psi K^{*} \mathrm{MC}$ samples, and that of the partner electrons $\left(E_{T}>5 \mathrm{GeV}\right)$ of $J / \psi \rightarrow e e$ candidates in the CDF data, after the final selection (except $\mathrm{Had} / E M$ ) optimized for $K^{*} \gamma$. Again, we note that the MC does not simulate underlying events. Since the $J / \psi$ events with $p_{T}>10 \mathrm{GeV} / c$ are dominated by $B$ decays rather than prompt $J / \psi$ 's, the $\mathrm{Had} / E M$ distribution for the $J / \psi \rightarrow e e$ candidates can be regarded as a model, probably conservative in terms of the $\mathrm{Had} / \mathrm{EM}$ efficiency, for $J / \psi K$ including effects from underlying events.

Our $\mathrm{Had} / E M$ cut depends on the number of the associated CTC tracks to the electron cluster $\left(<0.04\right.$ for $N_{t r k}=1$ and $<0.1$ for $\left.N_{t r k} \geq 2\right)$. The difference of the $N_{t r k}$ distribution between the signal and reference channels also affects the $\mathrm{Had} / E M$ cut 
efficiency. Figure 4.14 shows the $N_{t r k}$ distribution for the samples shown in Figure 4.13. We see that $45 \%$ (less than $1 \%$ ) of the partner electrons of $K^{*} \gamma(J / \pi K)$ are in the same tower as the trigger electrons, which makes the effective $\mathrm{Had} / E M$ cut for $K^{*} \gamma$ looser than that for $J / \psi K$.

The obtained MC efficiencies are $99.9 \%$ and $97.6 \%$ for $K^{*} \gamma$ and $J / \pi K$, respectively: it is not canceled in the relative efficiency but a $2 \%$ difference.

Given that the efficiency for the partner electrons of the $J / \psi \rightarrow e e$ candidates is $91 \%$ ( $6 \%$ lower than that for the $J / \psi K \mathrm{MC}$ ), the question is the reliability of the relative efficiency from the MC's.

We first estimate how the different effects from the hadrons from $B$ decays change the efficiency. Such effects are present in the $\mathrm{Had} / E M$ distribution even if we assume same effects from underlying events. We consider that the real $\mathrm{Had} / E M$ distribution for $K^{*}$ is close to that for the $J / \psi \rightarrow e e$ partner electrons in the CDF data. The efficiency difference between $K^{*} \gamma$ and $J / \psi K$ is then simulated by shifting the cut values (0.04 or 0.1 ) by a same amount at the same time. We take this shift to be twice the difference of the average $\mathrm{Had} / E M$ values between the $K^{*} \gamma$ and $J / \psi K \mathrm{MC}$ which is $\pm 0.005: 2 \times(0.0070-0.0043) \simeq 0.005$. The inserted arbitrary safety-factor of 2 is meant to cover possible incompleteness of the hadron shower simulation in the MC as well as the uncertainty of this scheme itself. Figure 4.15 shows the $\mathrm{Had} / \mathrm{EM}$ efficiency curves for the $J / \psi \rightarrow e e$ partner electrons with our cut values and their shifts. The result is $(91 \pm 6) \%$ (standard), $(90 \pm 6) \%(-0.005)$, and $(92 \pm 6) \%(+0.005)$. We expect that the different hadronic contents between the radiative and the $J / \psi$ decays have little effect to the $\mathrm{Had} / E M$ cut efficiency.

The real $N_{t r k}$ distribution for $K^{*} \gamma$ cannot be estimated as long as effects from underlying events are not correctly implemented in the MC. Rather than attempting to investigate such effects, we check the efficiency variation between the two extreme cases, $\mathrm{Had} / E M<0.04$ (completely $N_{t r k}=1$ ) and $\mathrm{Had} / \mathrm{EM}<0.1$ (completely $N_{t r k} \geq 2$ ),

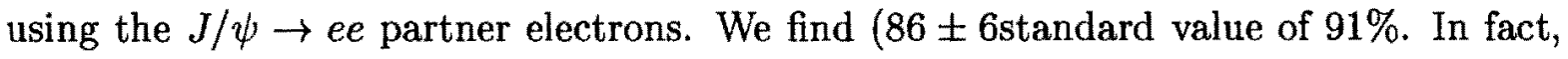
we expect more trigger electrons with $N_{t r k} \geq 2$ for $K^{*} \gamma$ than $J / \psi K$ and the difference would be somewhere between $0 \%$ and $+5 \%$ which covers the $+2 \%$ difference obtained from the MC. We, therefore, simply assign a $5 \%$ of the systematic uncertainty related to the relative $\mathrm{Had} / E M$ cut efficiency. 

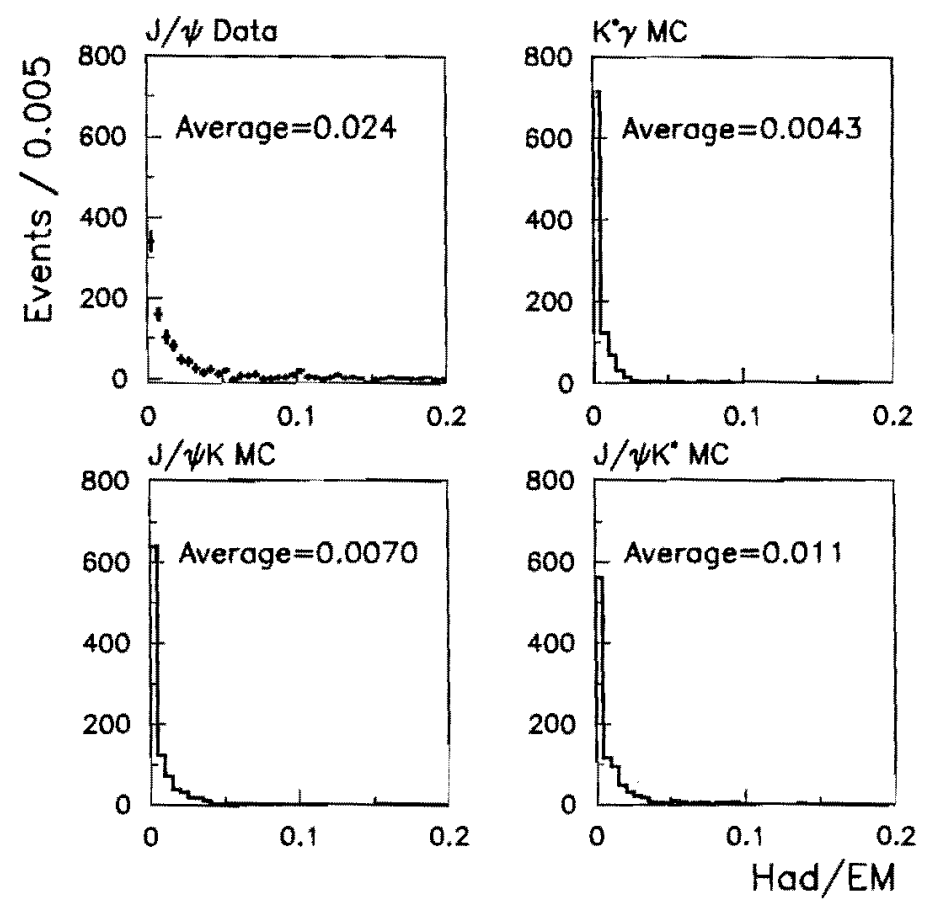

Figure 4.13: $\mathrm{Had} / E M$ distribution for partner electrons of the $J / \psi \rightarrow e e$ candidates and trigger electrons of the $K^{*} \gamma, J / \psi K$, and $J / \psi K^{*} \mathrm{MC}$.
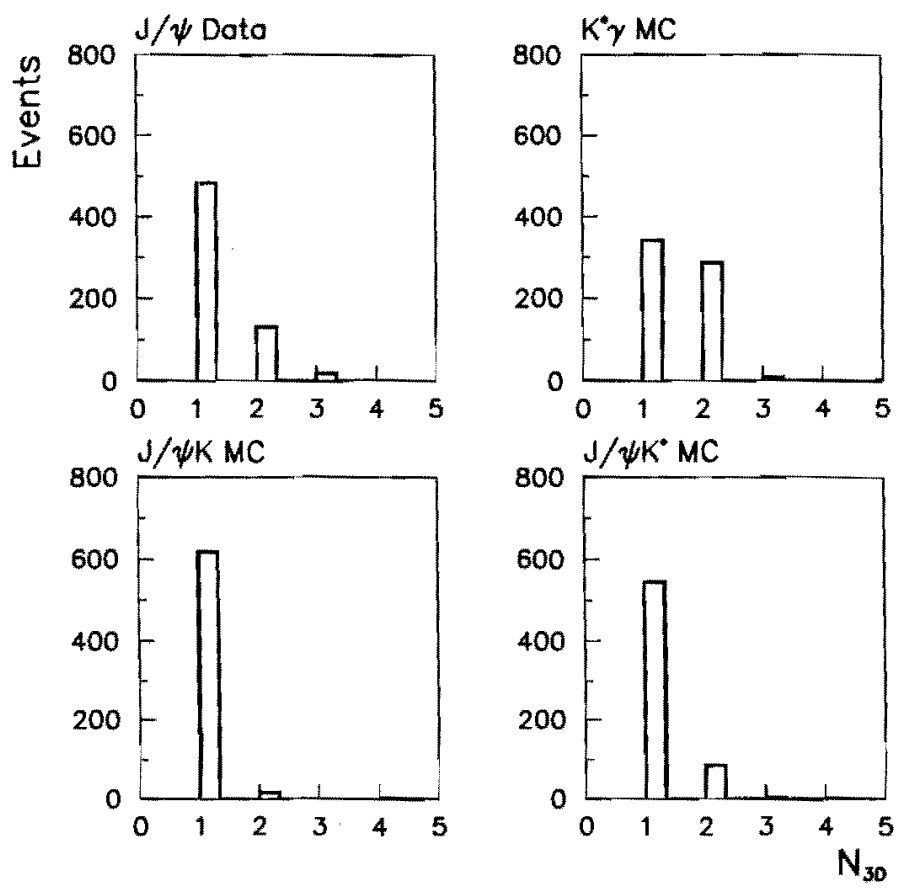

Figure 4.14: $N_{t r k}$ distribution for partner electrons of the $J / \psi \rightarrow e e$ candidates and trigger electrons of the $K^{*} \gamma, J / \psi K$, and $J / \psi K^{*}$ MC. 

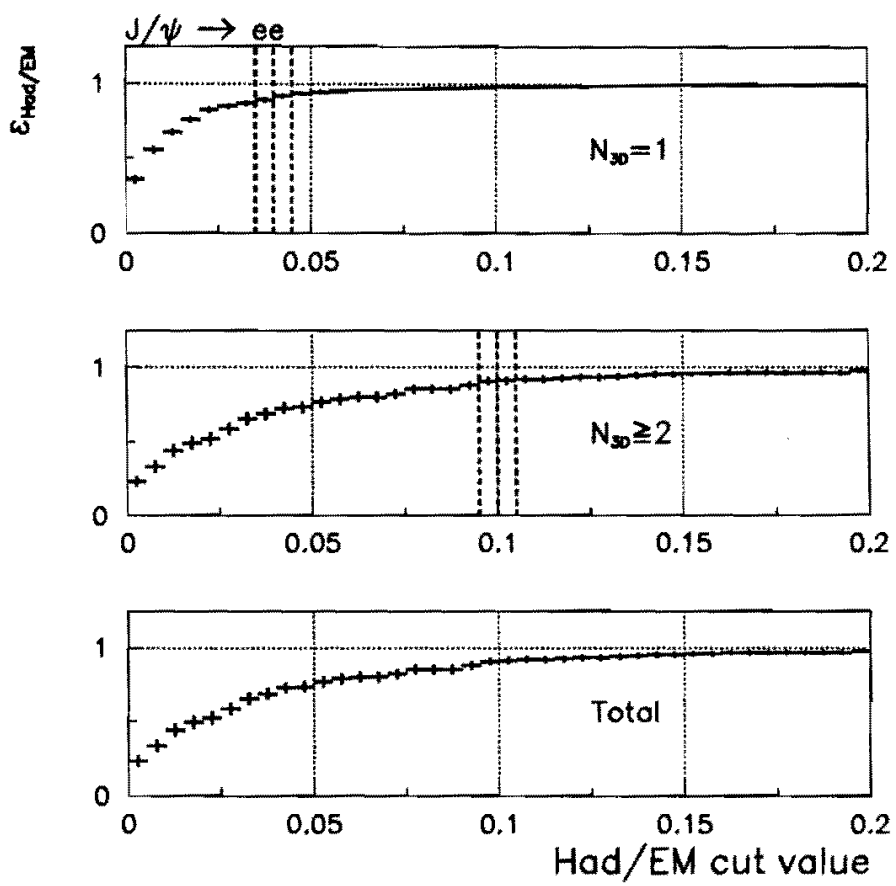

Figure 4.15: $\mathrm{Had} / \mathrm{EM}$ cut efficiency for partner electrons of the $J / \psi \rightarrow e e$ candidates

\subsection{Class 3 Efficiencies}

We assume the $B$ isolation cut efficiency $\left(\epsilon_{i s o}\right)$ is common between the radiative and $\bar{B}_{u} \rightarrow J / \psi K^{-}$decays, and it falls under class 3 . Figure 4.16 shows the $B$ mass distribution for the $\bar{B}_{u} \rightarrow J / \psi K^{-}$candidates in data before and after the isolation cut and background subtracted $I_{B}$ distribution. The efficiency is estimated to be $0.82 \pm 0.05$. The efficiency is expected to be $p_{T}(B)$ dependent, and Figure 4.17 shows the efficiency to be $0.80 \pm 0.07$ and $0.86 \pm 0.08$, respectively, for the candidates with $p_{T}(B)<20 \mathrm{GeV} / c$ and $p_{T}(B)>20 \mathrm{GeV} / c$. Figure 4.18 shows the $\mathrm{MC} p_{T}$ distribution of the $B$ hadrons after all the selection cuts. Note that our $\mathrm{MC}$ does not simulate the underlying events and the isolation cut efficiency is always 1 . The $p_{T}$ distributions are similar between the radiative decay and the reference signals, and it ensures the cancelation of the efficiency.

\subsection{Tracking Efficiencies}

The CTC $\left(\epsilon_{C T C}\right)$ and SVX $\left(\epsilon_{S V X}\right)$ tracking efficiencies spread over the classes. In this section, we discuss the details of the estimation of the tracking efficiciency. 


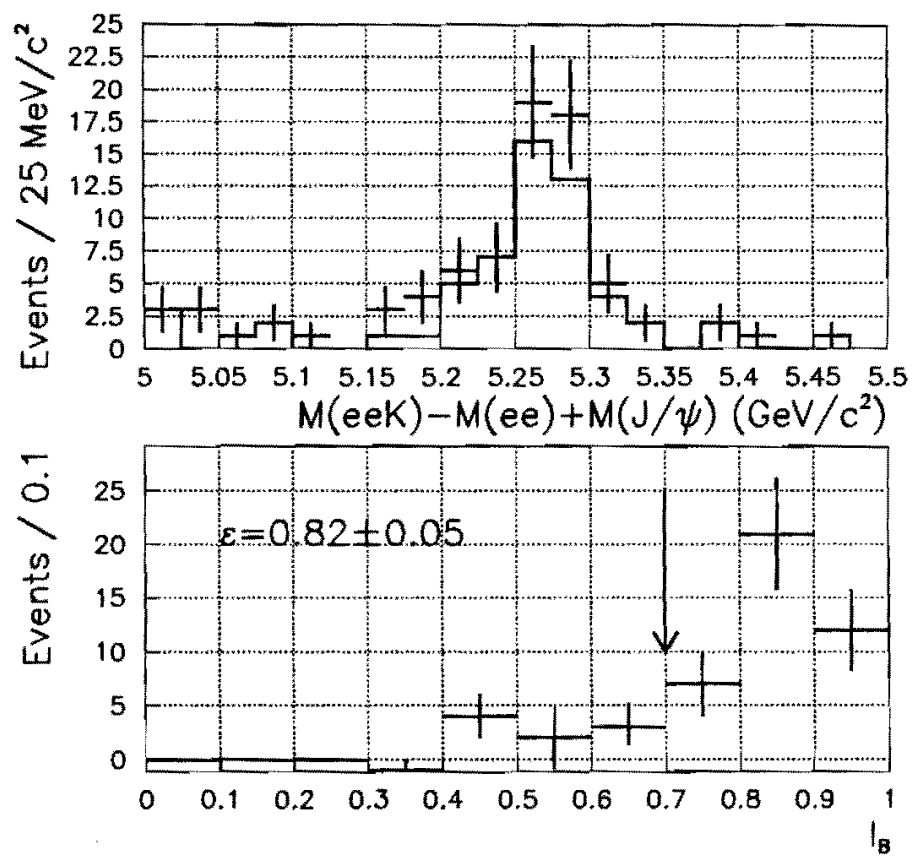

Figure 4.16: The $\bar{B}_{u}$ mass distributions before (points) and after (histogram) the $B$ isolation cut (top), and the $I_{B}$ distribution (bottom) obtained from the $\bar{B}_{u} \rightarrow J / \psi K^{-}$candidates in data. The arrow in the bottom plot corresponds to the cut value.

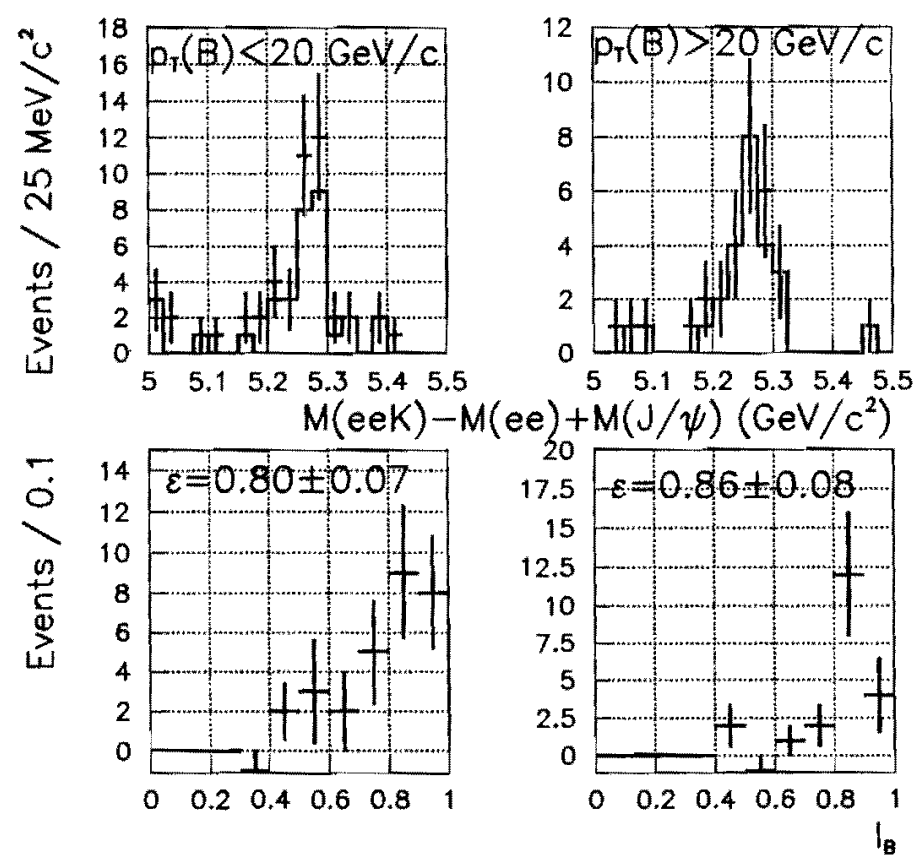

Figure 4.17: The same plots as the Figure 4.16 with differenc $p_{T}(B)$ regions, $p_{T}(B)<20 \mathrm{GeV} / c$ (left) and $p_{T}(B)<20 \mathrm{GeV} / c$ (right). 

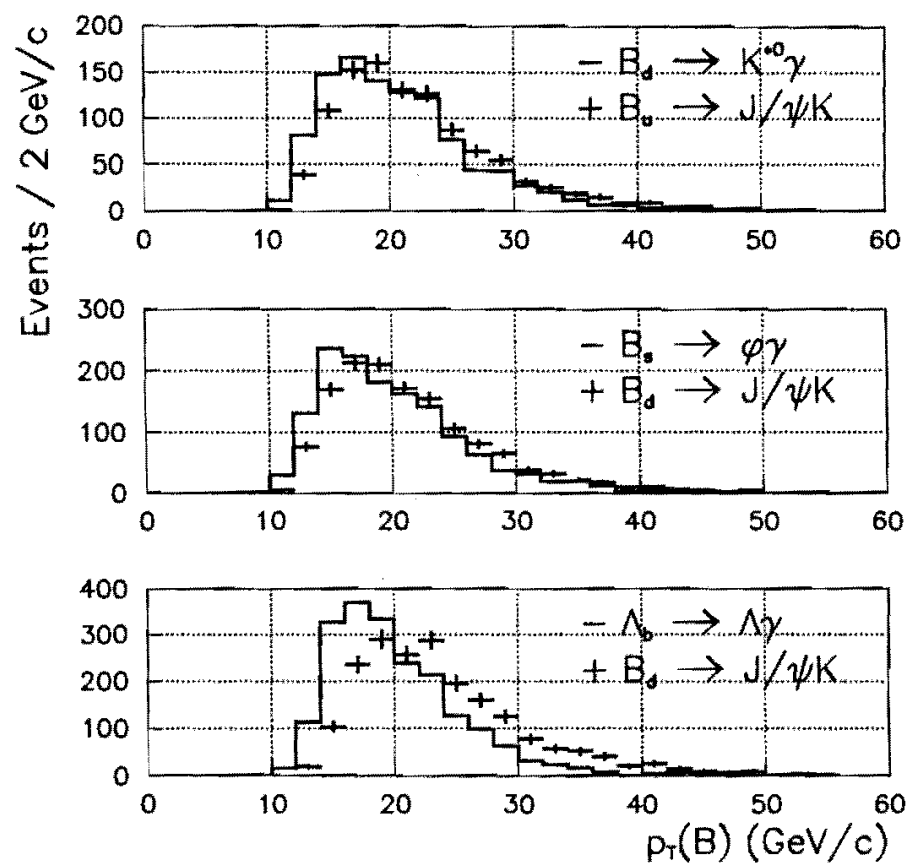

Figure 4.18: $p_{T}(B)$ distributions for the radiative $B$ decays (points) and the reference signals (histograms) after all the selection cut obtained from the MC sample.

\subsubsection{CTC Tracking Efficiencies}

\subsubsection{Overview}

The CTC tracking efficiency consists of the quality cut efficiency including the chamber hit efficiency, and the pattern recognition efficiency. For the estimation of the CTC track quality cut efficiency, we rely on $\mathrm{MC}$ calculation based on measured CTC hit efficiency in $\mathrm{CDF}$ data. On the other hand, our MC does not simulate the CTC pattern recognition, and we thus use an efficiency estimated by embedding simulated tracks in CDF data [41]. Figure 4.19 shows the tracking efficiency as a function of the transverse momentum. It rises quickly in the range $200<p_{T}<400 \mathrm{MeV} / c$, and has a plateau value for $p_{T}>$ $400 \mathrm{MeV} / c$ which depends on the instantaneous luminosity and the electric charge of tracks. The plateau value is estimated to be $0.96 \pm 0.02$. The radiative decays and $\bar{B}_{u} \rightarrow J / \psi K^{-}$are reconstructed with 4 and 3 tracks, respectively. The CTC tracking efficiencies for the conversion electrons and the electrons from $J / \psi$, and one of the hadron tracks from the radiative decays and the $K$ track from $\bar{B}_{u} \rightarrow J / \psi K^{-}$are similar. Thus the CTC track quality cut efficiencies for the three tracks fall under class 2 . We assume the CTC pattern recognition efficiencies are exactly canceled in the ratios (class 3 ). The CTC tracking efficiency for the second hadron track for the radiative decay falls under the class 1 . 
$97 / 12 / 11 \quad 14.50$

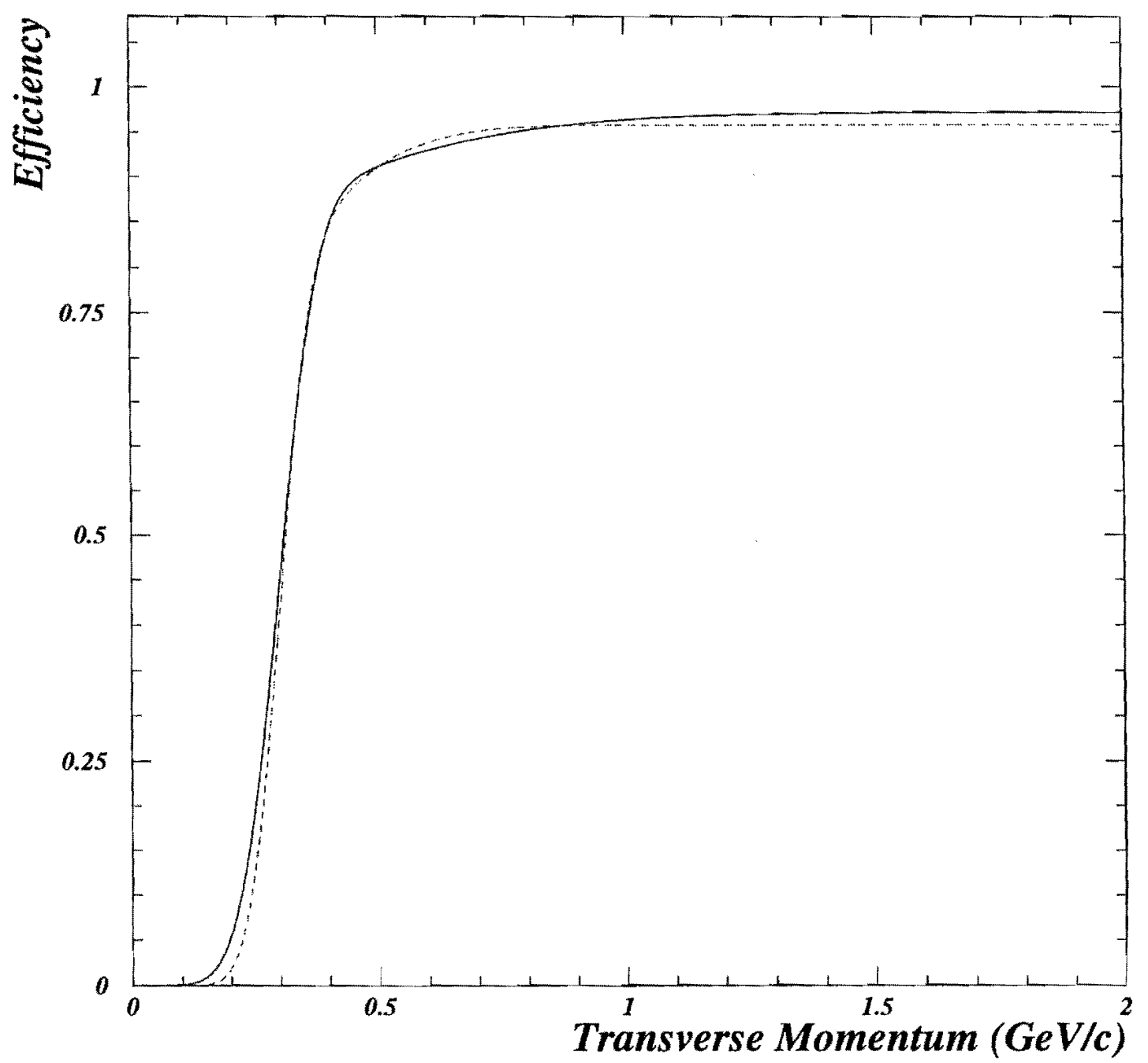

Figure 4.19: CTC track reconstruction efficiency as a function of the track $p_{T}$ for positively (solid line) and negatively (dashed line) charged tracks ( [41], Figure 5). 


\subsubsection{Conversion Tracking Efficiency}

The conversion occurs at the large radius ( 3 to $25 \mathrm{~cm}$ ) and the conversion electrons, especially for the low $p_{T}$ partner electrons, have a large impact parameter. We don't understand the CTC tracking efficiency for such tracks, because the CTC track is reconstructed with the chamber hit within a certain cone from the primary vertex. If there are sources of the inefficiency for the low $p_{T}$ and displaced tracks, the effect is not canceled in the ratio between the conversions and the $J / \psi \rightarrow e e$. Thus we checked the radius dependence of the conversion electron tracking efficiency. The inefficiency of the pattern recognition is caused by: (1) aging of the CTC which depends on elapsed time; (2) background hit which depends on the physics process and instantaneous luminosity.

We check the effect by using the conversion and the $J / \psi$ candidates themselves in the Run I data. We have typically $>500$ events of the photon conversion per one run, and we can estimate the conversion rate, (number of conversion candidates)/(integrated luminosity), run by run. The high $p_{T}(8 \mathrm{GeV} / c)$ conversion production rate itself does not depend on the CTC aging and the instantaneous luminosity, so if we see such dependence, it is caused by the reconstruction efficiency.

Figure 4.20 shows the integrated luminosity (top), averaged instantaneous luminosity (middle), and conversion rate (bottom) run by run which there are at least 5000 conversion candidates. The obtained conversion rate is around $10 \mathrm{nb}(8 \mathrm{nb})$ at the beginning (end) of the Run I, and it is mostly caused by the aging of the CTC. Note that the instantaneous luminosity of the Run I Tevatron was higher for the later runs, thus the two effects are correlated.

Figure 4.21 shows the conversion rate as a function of the instantaneous luminosity. Rates for the various $r$ region:

- SVX: $3<r<11 \mathrm{~cm}$,

- VTX: $12<r<21 \mathrm{~cm}$,

- CTC can: $22<r<30 \mathrm{~cm}$,

are shown. The obtained conversion rate is $\sim 40 \%$ lower at a $2 \times 10^{31} \mathrm{~cm}^{-2} \mathrm{~s}^{-1}$ of the instantaneous luminosity. Again we note that this is correlated with the effect of the CTC aging.

An important note is that both the CTC aging and the background hit effect are physics process and the selection cut dependent. For example our conversion candidates are dominated by $\pi^{0}$ in the QCD jets and expected to accompanied by much more 
background tracks than the $b \bar{b}$ production. Also the isolation cut which was required for the $B$ candidates reduces the background hits. So the inefficiency for the CTC tracking for the $B$ candidates is expected to be smaller, and it is similar between the radiative $B$ decay and $\bar{B}_{u} \rightarrow J / \psi K^{-}$channels. However if the effect depends on the radius of the conversion vertex which we discussed before, it is not canceled in the ratio between the radiative $B$ decay channels and the reference $\bar{B}_{u} J / \psi K^{-}$channel $(r=0)$.

Figure 4.22 shows the run and instantaneous luminosity dependence of the fraction of the conversion rate in the CTC can. The distributions are flat, i.e., the conversion tracking efficiency is expected be canceled in the ratio between the conversion and the $J / \psi$.

A further check is available by using the $J / \psi$ rate in the data, however, the statistics is not enough to check it run by run, thus we divide the data into the following 6 periods.

$\begin{array}{ccc}\text { period } & \text { run range } & \text { Luminosity }\left(\mathrm{pb}^{-1}\right) \\ \text { a } & <60000 & 6.3 \\ \text { b } & 60000-62500 & 11.4 \\ \text { c } & 62500-65000 & 17.7 \\ \text { d } & 65000-67000 & 15.7 \\ \text { e } & 67000-70000 & 17.2 \\ \text { f } & >70000 & 9.4\end{array}$

Figure 4.23 shows the $J / \psi$ mass distribution for the six run periods, and the obtained $J / \psi$ rate is shown in Figure 4.24. The run dependence of the conversion rate is also shown. We see similar structure between the conversion and $J / \psi$ rates.

\subsubsection{3 $\Lambda$ Tracking Efficiency}

Our assumption was that the tracking efficiency between one of the decay daughter tracks of the strange meson $\left(K^{*}, \phi\right.$, and $\left.\Lambda\right)$ from the radiative $B$ signal and the kaon from the $\bar{B}_{u} \rightarrow J / \psi K^{-}$was canceled, and the efficiency for the other daughter tracks was (96士2)\% categorized into class 1 . In the case of $\Lambda_{b} \rightarrow \Lambda \gamma$, we can write as

$$
\frac{\epsilon_{C T C}(\Lambda)}{\epsilon_{C T C}(K)}=\epsilon_{C T C}(\pi)=(96 \pm 2) \% .
$$

However, $\Lambda$ has a largely displaced vertex and there are similar uncertainty of the tracking efficiency as the ratio between the conversion electrons and $J / \psi \rightarrow e e$. If there is such a radius dependence, the class 1 tracking efficiency can differ from the $(96 \pm 2) \%$. 

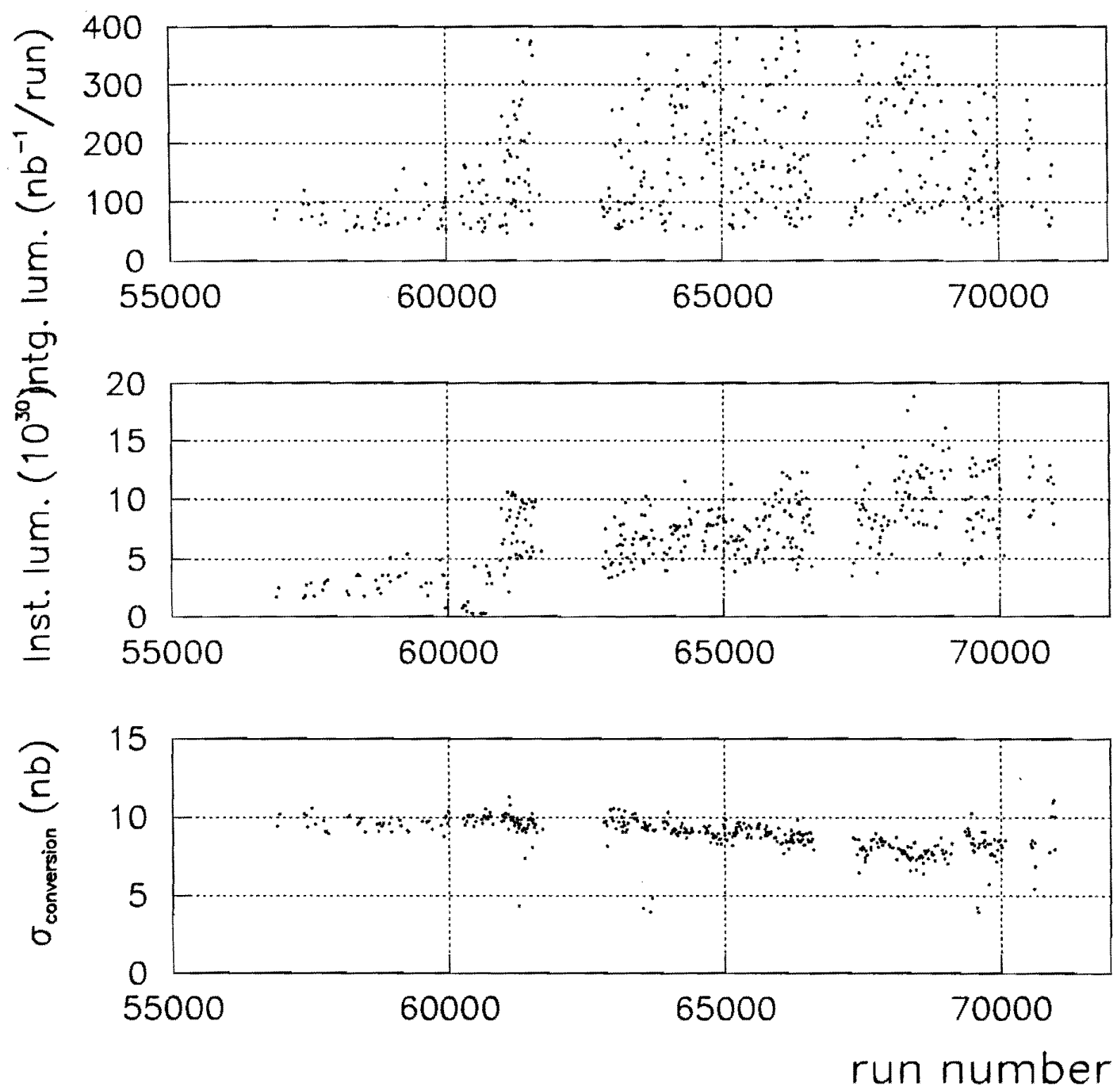

Figure 4.20: Integrated luminosity, instantaneous luminosity, and conversion rate run by run for those runs in which there are at least 500 conversion candidates. 

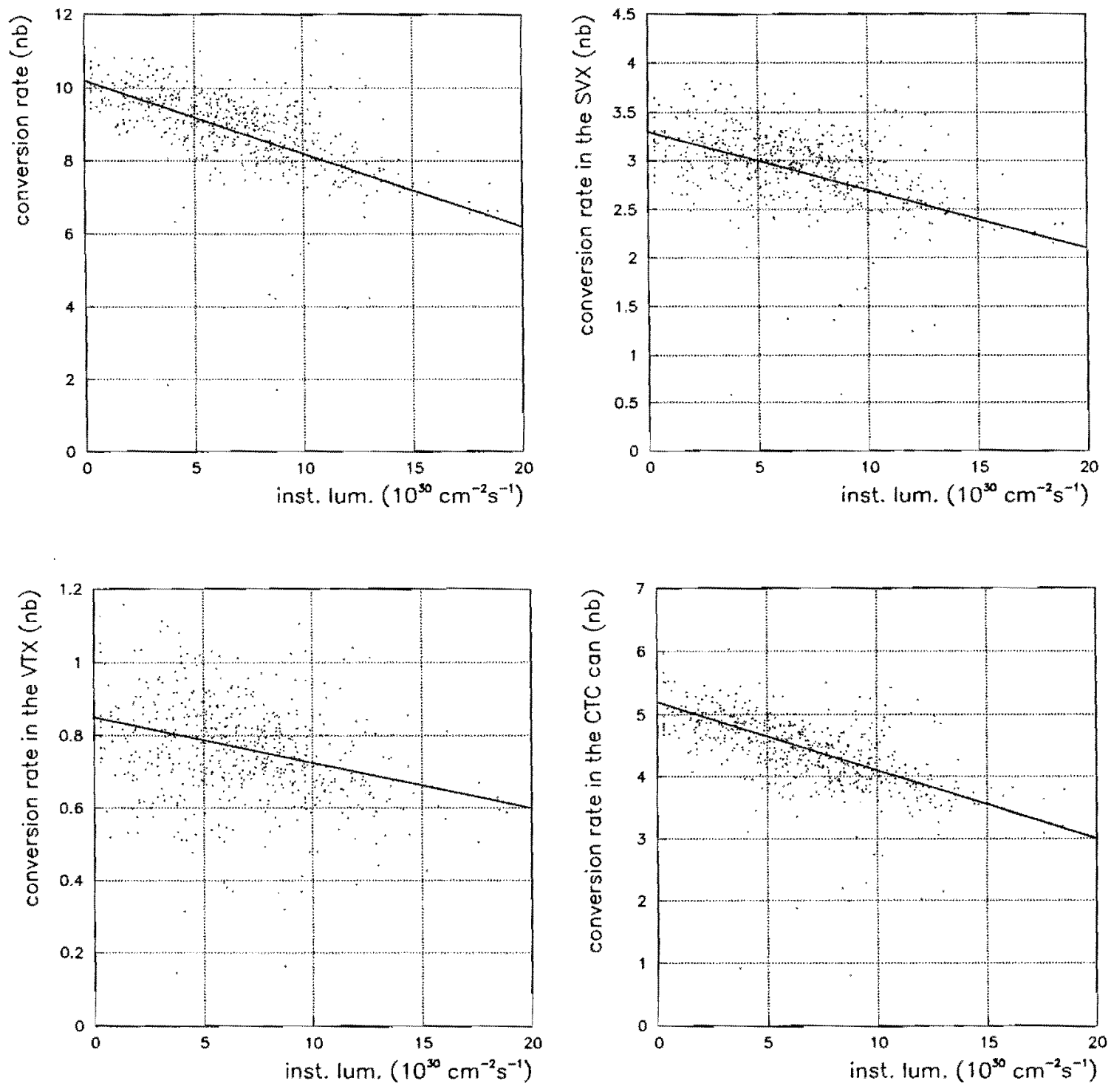

Figure 4.21: Scatter plots for the conversion rate in the various $r$ region as a function of the instantaneous luminosity. 

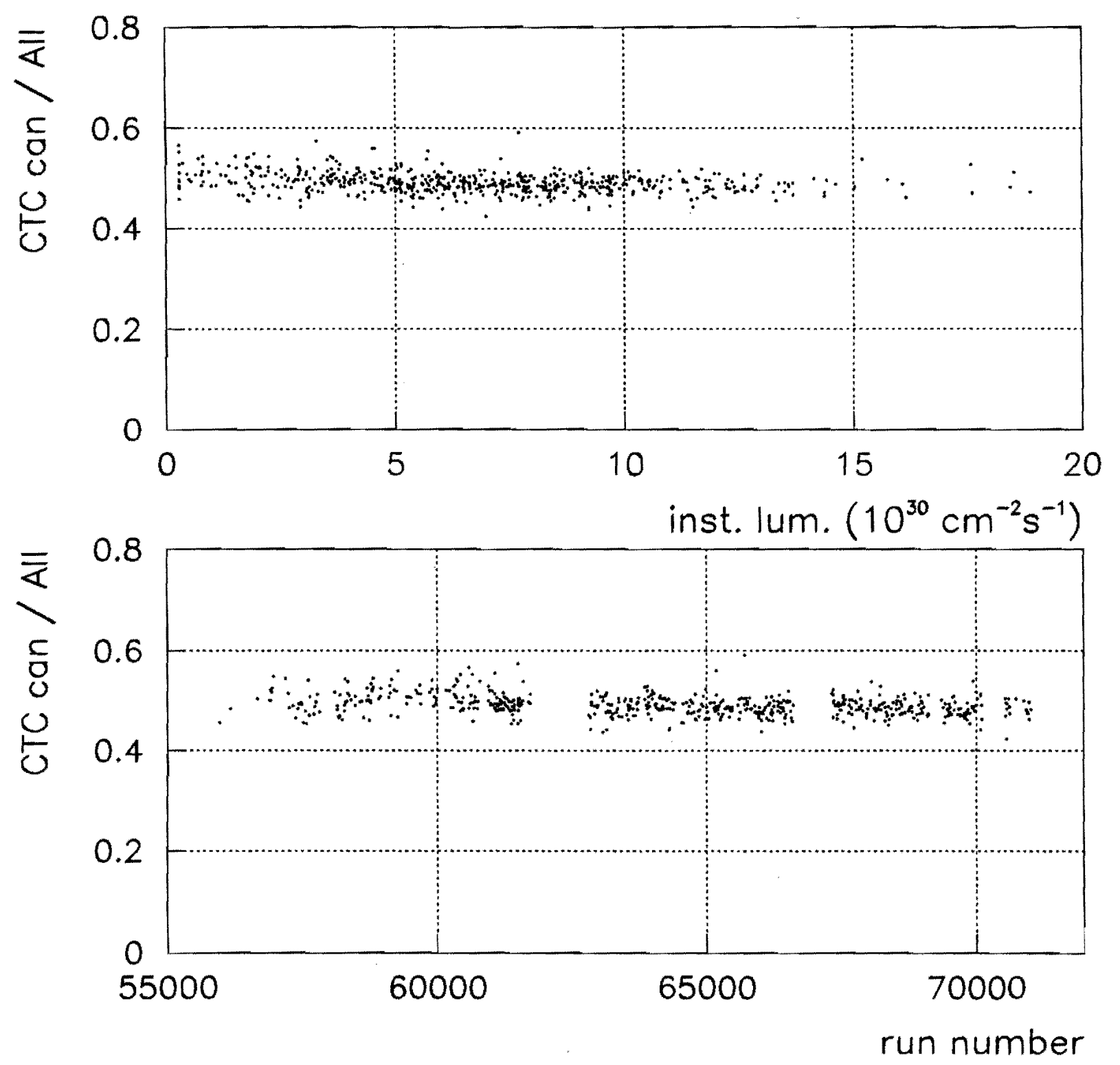

Figure 4.22: Fraction of the conversion rate in the CTC can as a function of the instantaneous luminosity (top) and the run number (bottom). 

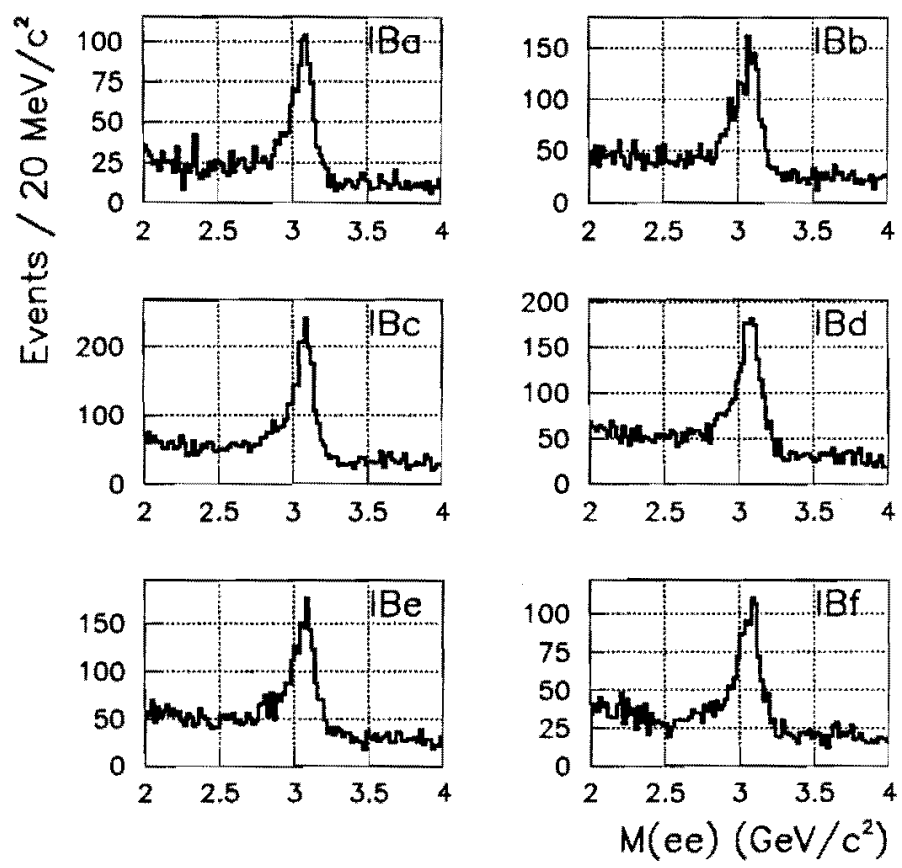

Figure 4.23: Dielectron mass distributions for the $J / \psi \rightarrow$ ee candidates for the six different run periods.
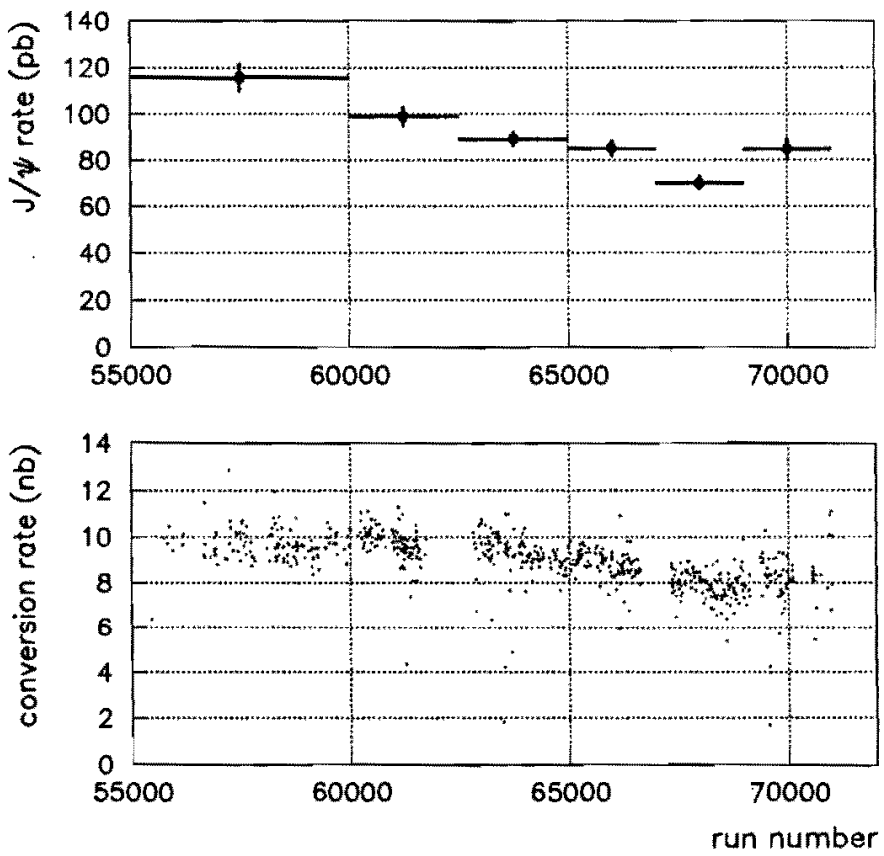

Figure 4.24: The $J / \psi \rightarrow e e$ and conversion rate a run by run. 

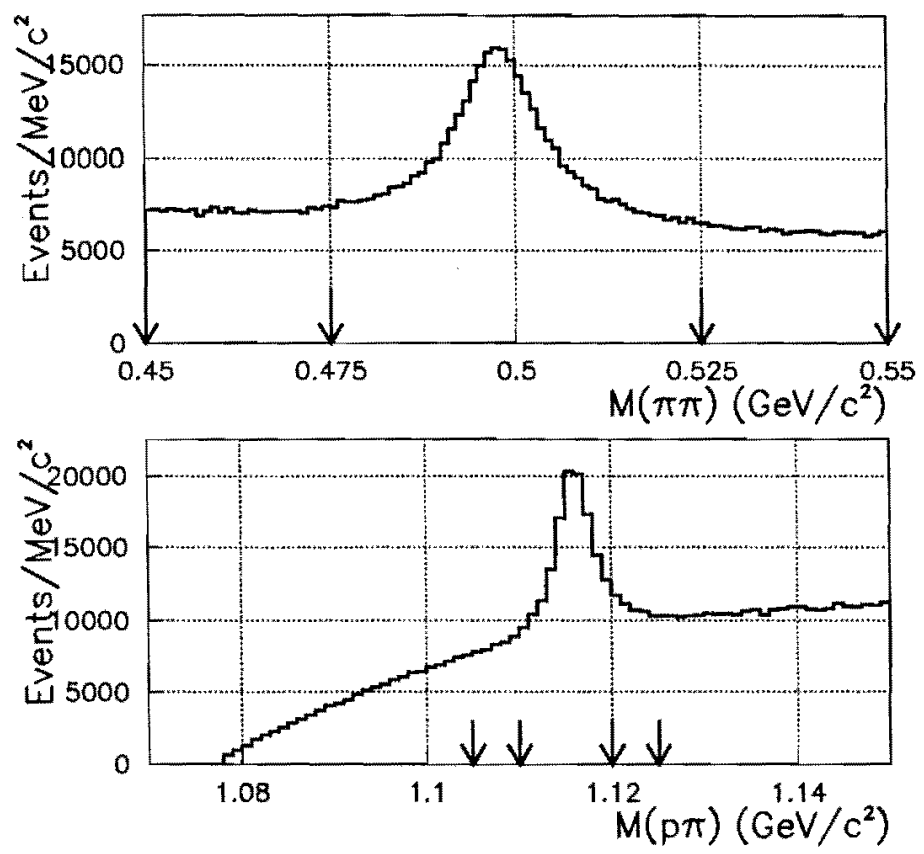

Figure 4.25: The $\Lambda$ and $K_{S}^{0}$ candidates in the data.

We check this by using the $\Lambda \rightarrow p \pi$ and also the $K_{S}^{0} \rightarrow \pi \pi$, which also has a displaced vertex, in the electron trigger data set. Figure 4.25 shows the $p \pi$ and $\pi \pi$ mass distribution for the $\Lambda$ and $K_{S}^{0}$ candidates reconstructed with CTC tracks after requiring single track $p_{T}>0.4 \mathrm{GeV} / c$, system $p_{T}>2 \mathrm{GeV} / c$, common vertex fitting probability $>0.1 \%$ and transverse decay length $>1 \mathrm{~cm}$. Note that the production rate itself of the low $p_{T}(\sim 2 \mathrm{GeV} / c) \Lambda$ and $K_{S}^{0}$ depends on the instantaneous luminosity, because of the contribution from other $p \bar{p}$ collisions in a single bunch crossing. Figure 4.26 shows the impact parameter (top) and $z$ (bottom) distribution of the neutral $\Lambda$ and $K_{S}^{0}$ track around the trigger electron primary vertex. Two histograms correspond to the events in the two track mass signal region and the side band region which are shown as arrows in Figure 4.25. By subtracting the two histograms, we obtain real distributions which are shown as points in Figure 4.26. The flat tail in the $z$ distribution corresponds to the contribution from the other $p \bar{p}$ collisions. After cutting on $|z|<5 \mathrm{~cm}$, we can assume the $\Lambda$ and $K_{S}^{0}$ production rates do not depend on the instantaneous luminosity, and then, we can do the similar checks as the conversion electrons.

Figure 4.27 shows the vertex radius distributions for the $\Lambda$ and $K_{S}^{0}$ candidates. The same background subtraction as Figure 4.26 is applied. Because of the longer lifetime for the $\Lambda(\sim 9 \mathrm{~cm})$ than the $K_{S}^{0}(\sim 3 \mathrm{~cm})$, the average decay length is longer for the $\Lambda$. Figure 4.28 shows the obtained $\Lambda$ and $K_{S}^{0}$ rate run by run for those runs in which there are at least $50 \Lambda$ and $K_{S}^{0}$. The ratio between the $\Lambda$ and $K_{S}^{0}$ is also shown. We see the 

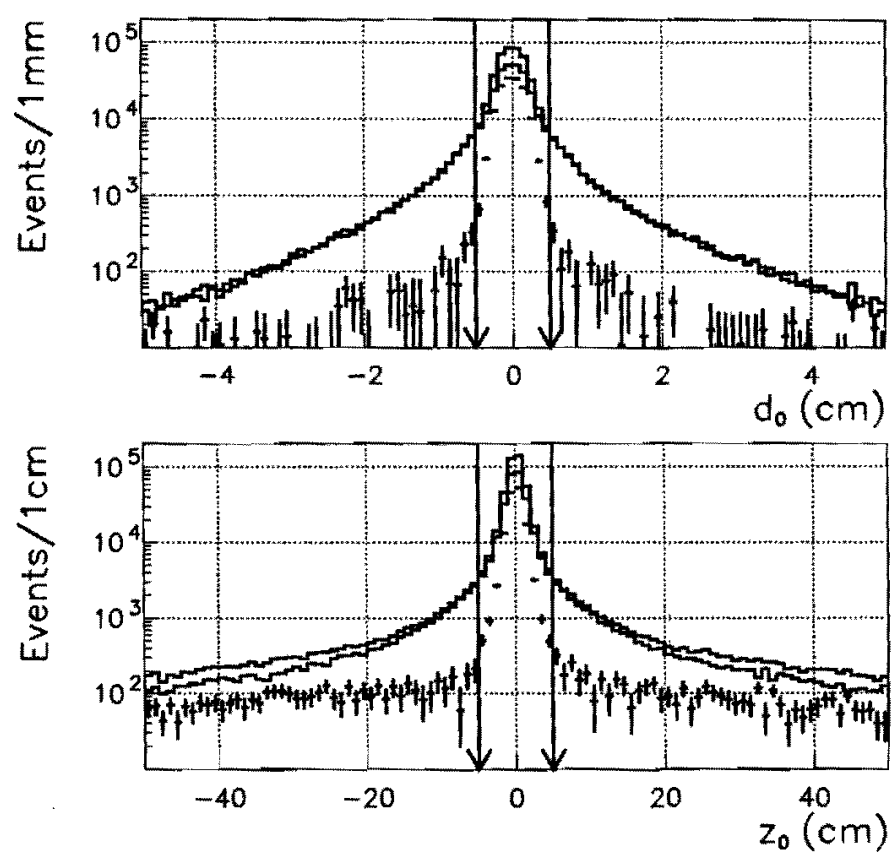

Figure 4.26: Impact parameter and $z$ distribution of the $\Lambda$ candidates around the trigger electron primary vertex.

$\Lambda$ and $K_{S}^{0}$ rates are decreased by $\sim 30 \%$ in the end of Run I, but their ratio is stable. Figure 4.29 shows the same plots as Figure 4.28 but shows the instantaneous luminosity dependence. The ratio between the $\Lambda$ and $K_{S}^{0}$ shows no luminosity dependence.

Figures 4.30 and 4.31 show the run and luminosity dependence of the fraction of the $\Lambda \rightarrow p \pi$ events with radius $>20 \mathrm{~cm}$. Around $30 \%$ of the $\Lambda$ 's have radius $>20 \mathrm{~cm}$, and we see no run and luminosity such dependence. This confirms we can use the single track CTC efficiency of $(96 \pm 2) \%$ for the the ratio of the tracking efficiency between the $\Lambda$ and $K_{S}^{0}$.

\subsubsection{SVX Tracking Efficiencies}

The SVX tracking efficiency is decomposed into the quality cut efficiency including the layer hit efficiency and the $\chi^{2}$ cut efficiency, and the pattern recognition efficiency. Both types of efficiencies are estimated by the detector simulation. This incorporates a measured hit efficiency in CDF data, and the reconstruction is done in the same way as data. All the tracks of $\bar{B}_{u} \rightarrow J / \psi K^{-}$are reconstructed using the SVX tracks. For the radiative decays, both of the conversion electrons are reconstructed with CTC tracks and the hadron tracks are reconstructed with SVX tracks except the CTC- $\Lambda_{b}$ candidate. Thus, the SVX tracking efficiencies for one of the hadron tracks from the radiative decays except the CTC- $\Lambda_{b}$ and the for $K$ track from $\bar{B}_{u} \rightarrow J / \psi K^{-}$are similar (class 2). The 


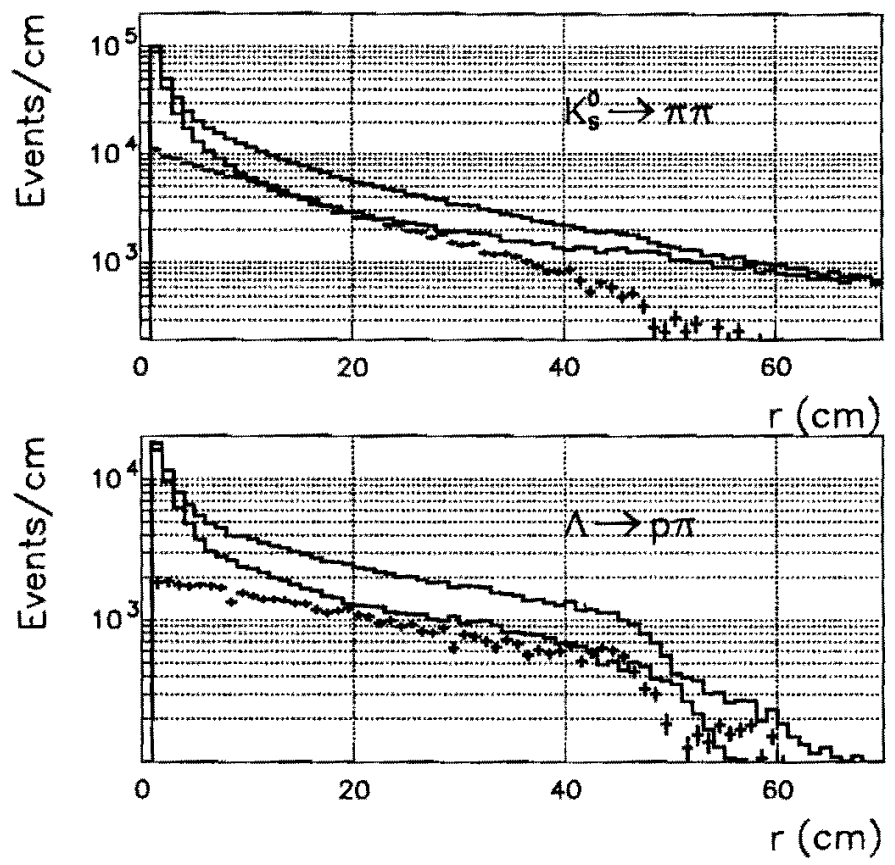

Figure 4.27: Vertex radius distributions for the $\Lambda$ and $K_{S}^{0}$ candidates in the strange mass signal and side band events (histograms) in the data. The points show the radius distribution after background subtraction using the side band events.
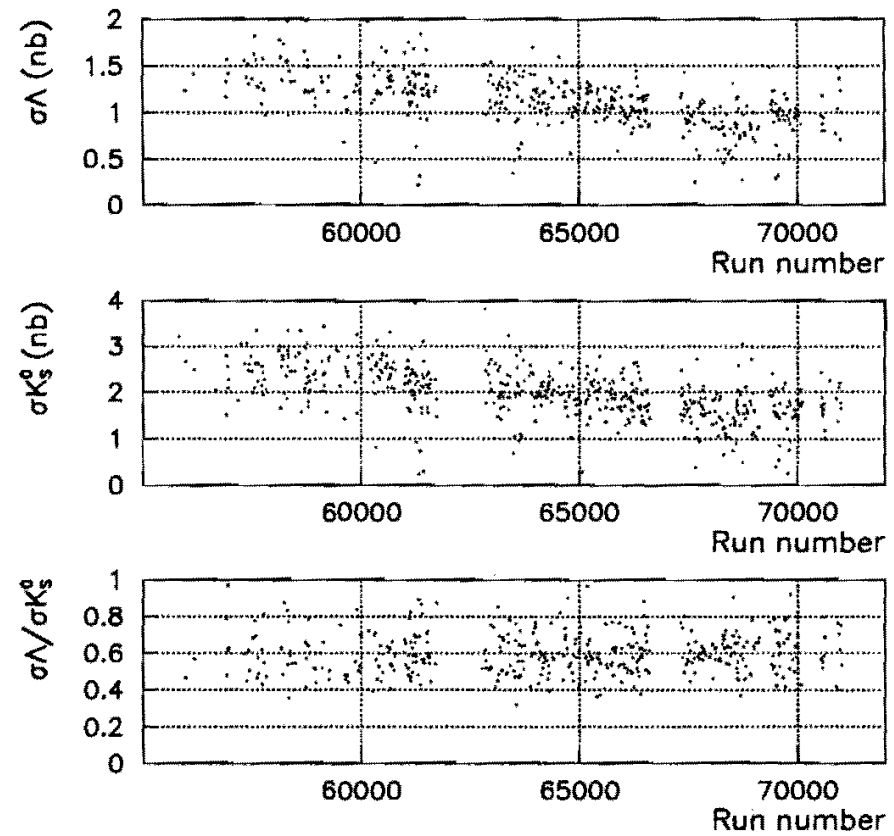

Figure 4.28: Run by run rates of the $\Lambda \rightarrow p \pi$ and $K_{S}^{0}$ events and teir ratio. 

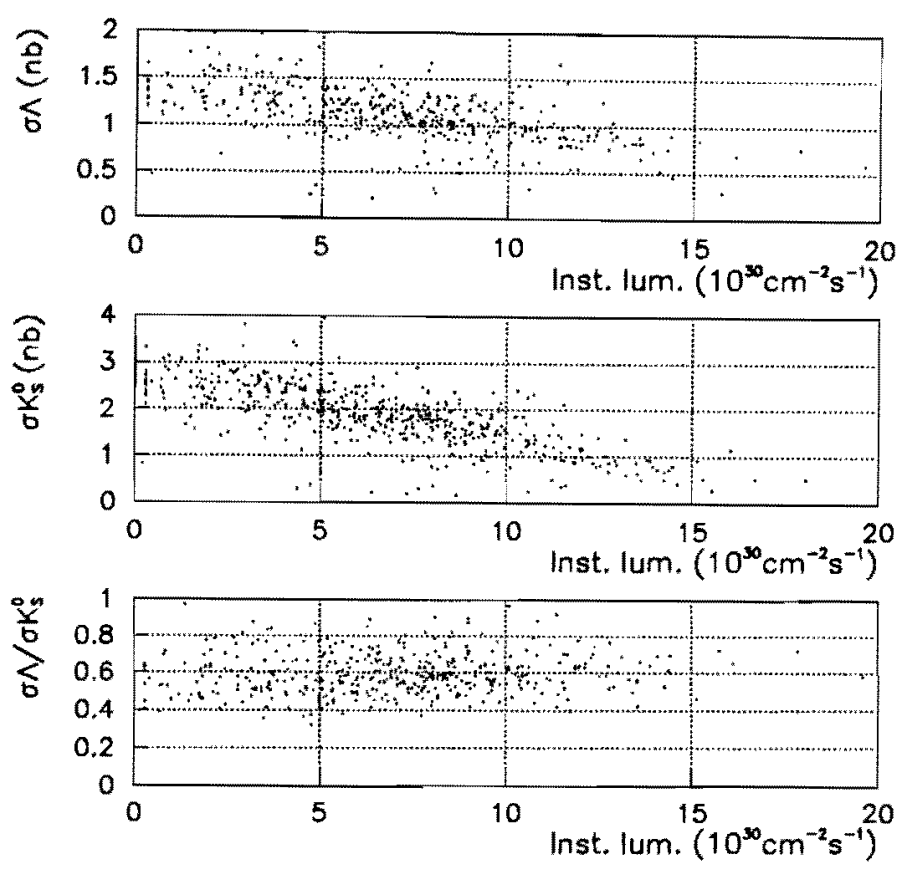

Figure 4.29: Instantaneous luminosity dependence of the $\Lambda \rightarrow p \pi$ and $K_{S}^{0}$ rates and their ratio.
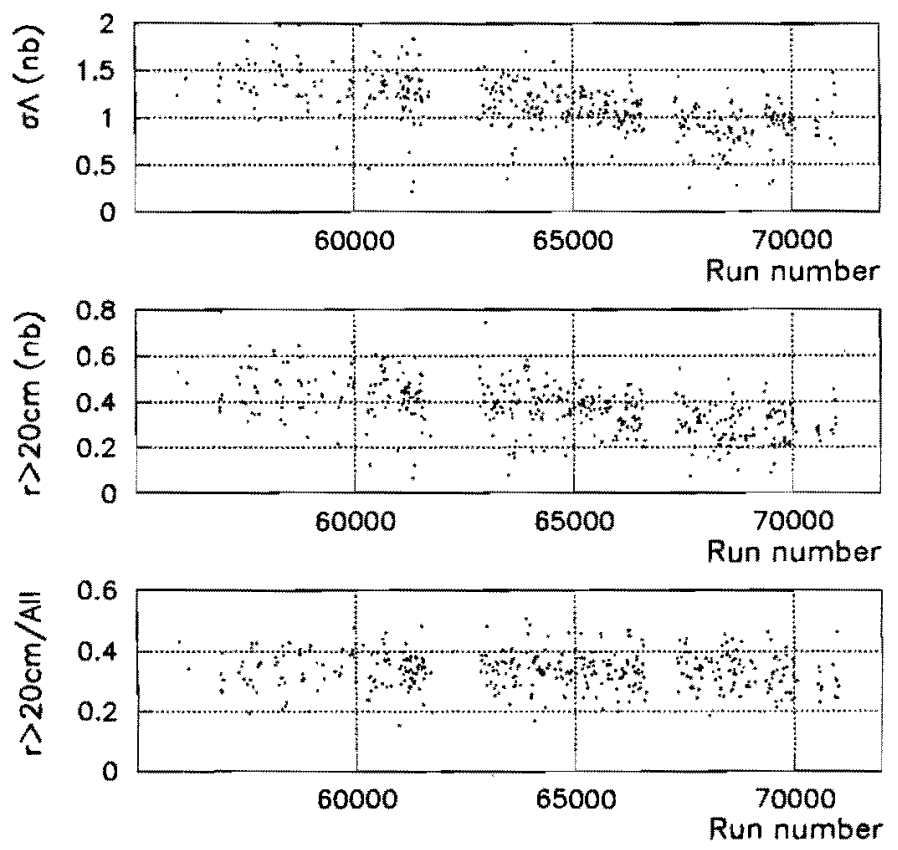

Figure 4.30: Run by run rate of the $\Lambda \rightarrow p \pi$ events and that with decay radius $>20 \mathrm{~cm}$, and their ratio. 

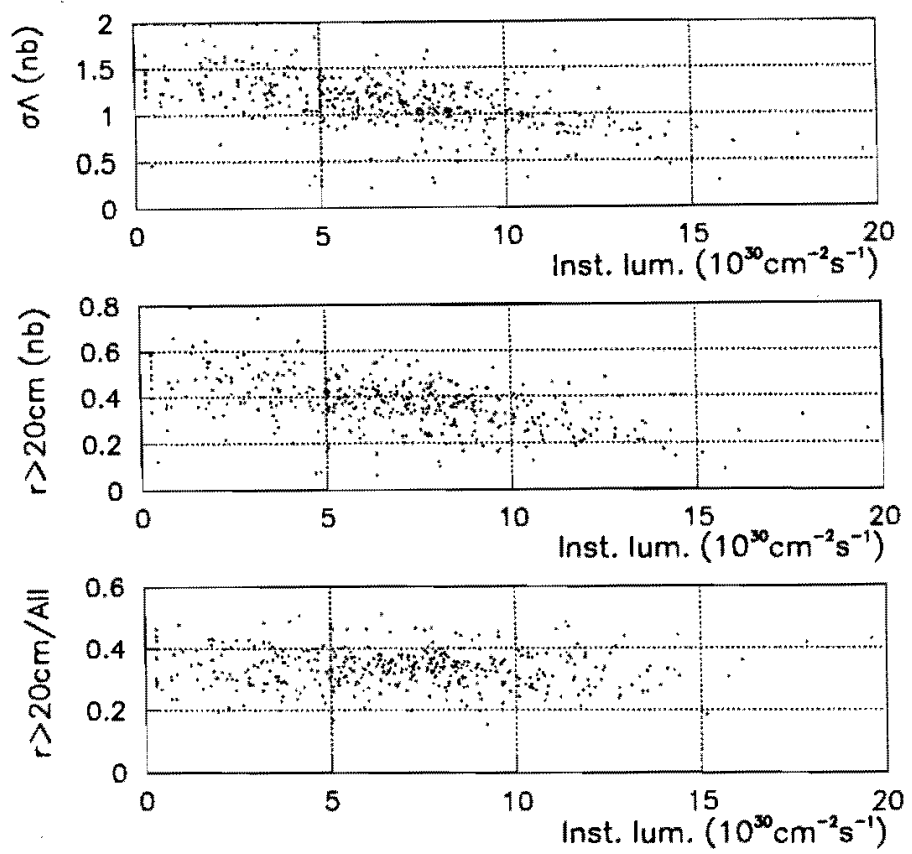

Figure 4.31: Instantaneous luminosity dependence of the $\Lambda \rightarrow p \pi$ events and that with decay radius $>20 \mathrm{~cm}$, and their ratio.

SVX tracking efficiencies for the rest of the tracks fall under class 1.

\subsubsection{Check of the SVX Efficiency}

We estimate the SVX efficiency by using the $J / \psi \rightarrow e e$ candidates, and compare with that of the $J / \psi K$ signal MC. We select the $J / \psi \rightarrow e e$ candidate with $\left|Z_{P V X}\right|<30 \mathrm{~cm}$, where $Z_{P V X}$ is the closest vertex to the $J / \psi \rightarrow e e$ vertex. If both of the CTC tracks have SVX tracks with at least 3 hits, the $J / \psi$ candidate is accepted as a SVX $J / \psi$ candidate. The efficiency is estimated by fitting the dielectron mass distribution to the two Gaussian + polinomial. The efficiency as a function of the vertex $z$ position is also estimated. Then, the background is subtracted by using the $M_{e e}$ side band events. We also estimate the efficiency from the $J / \psi K$ signal MC sample. Figure 4.32 shows the result. The efficiency for $J / \psi K$ MC sample is $(70 \pm 1) \%$, and that for the $J / \psi \rightarrow e e$ candidates is $(67 \pm 2) \%$. The MC simulation is sufficiently good for estimating the SVX efficiency. 

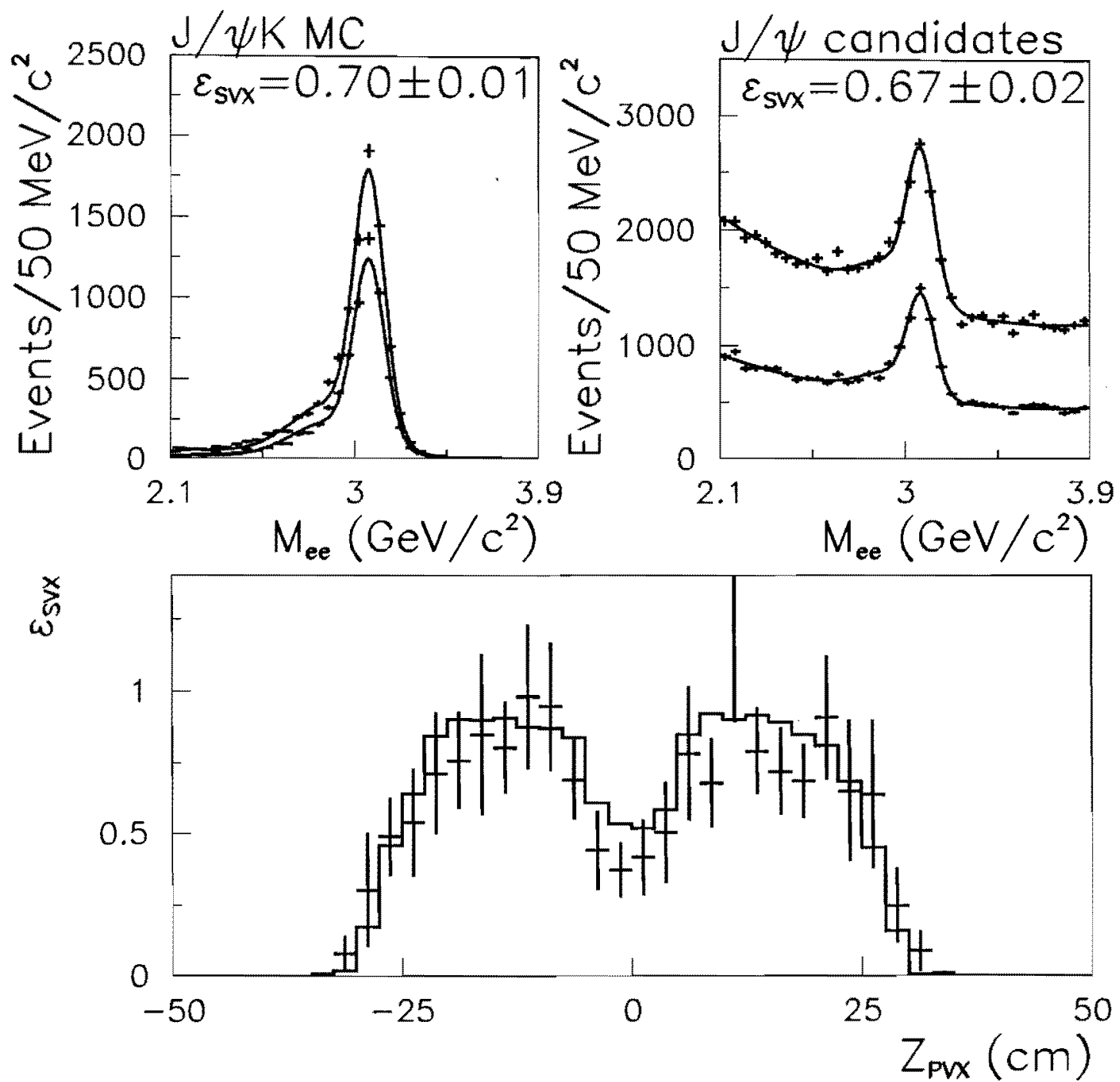

Figure 4.32: The SVX efficiency for the $J / \psi \rightarrow e e$ candidates and the $J / \psi K$ signal MC sample. 


\subsubsection{Vertex Fitting Efficiency}

\subsubsection{Overview}

The vertex fitting efficiencies for each decay channel are decomposed as follows:

$$
\begin{aligned}
\epsilon_{f i t}\left(K^{*} \gamma\right) & =\epsilon_{v t x}(\gamma) \cdot \epsilon_{v t x}\left(K^{*}\right) \cdot \epsilon_{\text {point }}\left(\gamma \rightarrow K^{*}\right) \cdot \epsilon_{\text {point }}\left(K^{*} \gamma \rightarrow \mathrm{PVX}\right), \\
\epsilon_{f i t}(\phi \gamma) & =\epsilon_{v t x}(\gamma) \cdot \epsilon_{v t x}(\phi) \cdot \epsilon_{\text {point }}(\gamma \rightarrow \phi) \cdot \epsilon_{\text {point }}(\phi \gamma \rightarrow \mathrm{PVX}), \\
\epsilon_{f i t}(\Lambda \gamma: \mathrm{CTC}) & =\epsilon_{v t x}(\gamma) \cdot \epsilon_{v t x}(\Lambda: \mathrm{CTC}) \cdot \epsilon_{\text {point }}(\gamma \rightarrow \mathrm{PVX}) \cdot \epsilon_{\text {point }}(\Lambda \rightarrow \mathrm{PVX}), \\
\epsilon_{f i t}(\Lambda \gamma: \mathrm{SVX}) & =\epsilon_{v t x}(\gamma) \cdot \epsilon_{v t x}(\Lambda: \mathrm{SVX}) \cdot \epsilon_{\text {point }}(\gamma \rightarrow \mathrm{PVX}), \\
\epsilon_{f i t}(\mathrm{~J} / \psi K) & =\epsilon_{v t x}(J / \psi) \cdot \epsilon_{v t x}(\mathrm{~J} / \psi K) \cdot \epsilon_{\text {point }}(\mathrm{J} / \psi K \rightarrow \mathrm{PVX}),
\end{aligned}
$$

where,

$\epsilon_{v t x}(\gamma) \quad:$ conversion constraint fit,

$\epsilon_{v t x}(X) \quad: X$ common vertex constraint fit,

$\epsilon_{\text {point }}(X \rightarrow Y): X \rightarrow Y$ pointing constraint fit.

The efficiency for the fitting CL $>0.1 \%$ cut is $99.9 \%$ by definition, however, we do not understand the the error matrix of the track helix parameters very well, and the efficiency can be different. It is known that the effect is significant for the largely displaced tracks. Thus we need to check the conversion and $\Lambda$ vertex fit.

Not only the tracking detector resolution, but also the multiple scattering, ionization loss, and bremsstrahlung, contribute to the error matrix. In the vertex constraint fit, we take account of the multiple scattering and ionization, but don't take account of the bremsstrahlung and it increases the fitting $\chi^{2}$. Thus we should take need to check the conversion and $J / \psi$ vertex fit efficiency.

\subsubsection{Conversion Constraint Fitting Efficiency}

As we discussed briefly in Section 4.2 and Appendix A, the error matrix of the CTC track for the large radius conversion electron is underestimated and the fitting $\chi^{2}$ of the conversion constraint is overestimated. Thus the fitting CL cut efficiency $\left(\epsilon_{v t x}(\gamma)\right)$ which should be 1-CL by definition can have $r$ dependence and differ from that for the reference $J / \psi$ channel $\left(\epsilon_{v t x}(J / \psi)\right.$, where our MC does not simulate the effect.

Figure 4.33 shows radius distributions of the conversion vertex for three different fitting CL regions, $\mathrm{CL}<10^{-30}$ (top), $10^{-30}<\mathrm{CL}<10^{-3}$ (middle), and CL $>10^{-3}$ (bottom). There are clear excess in the radius $\sim 25 \mathrm{~cm}$, which corresponds to the CTC can, in the middle plot. This shows clear evidence of the inefficiency for the CL cut, and 

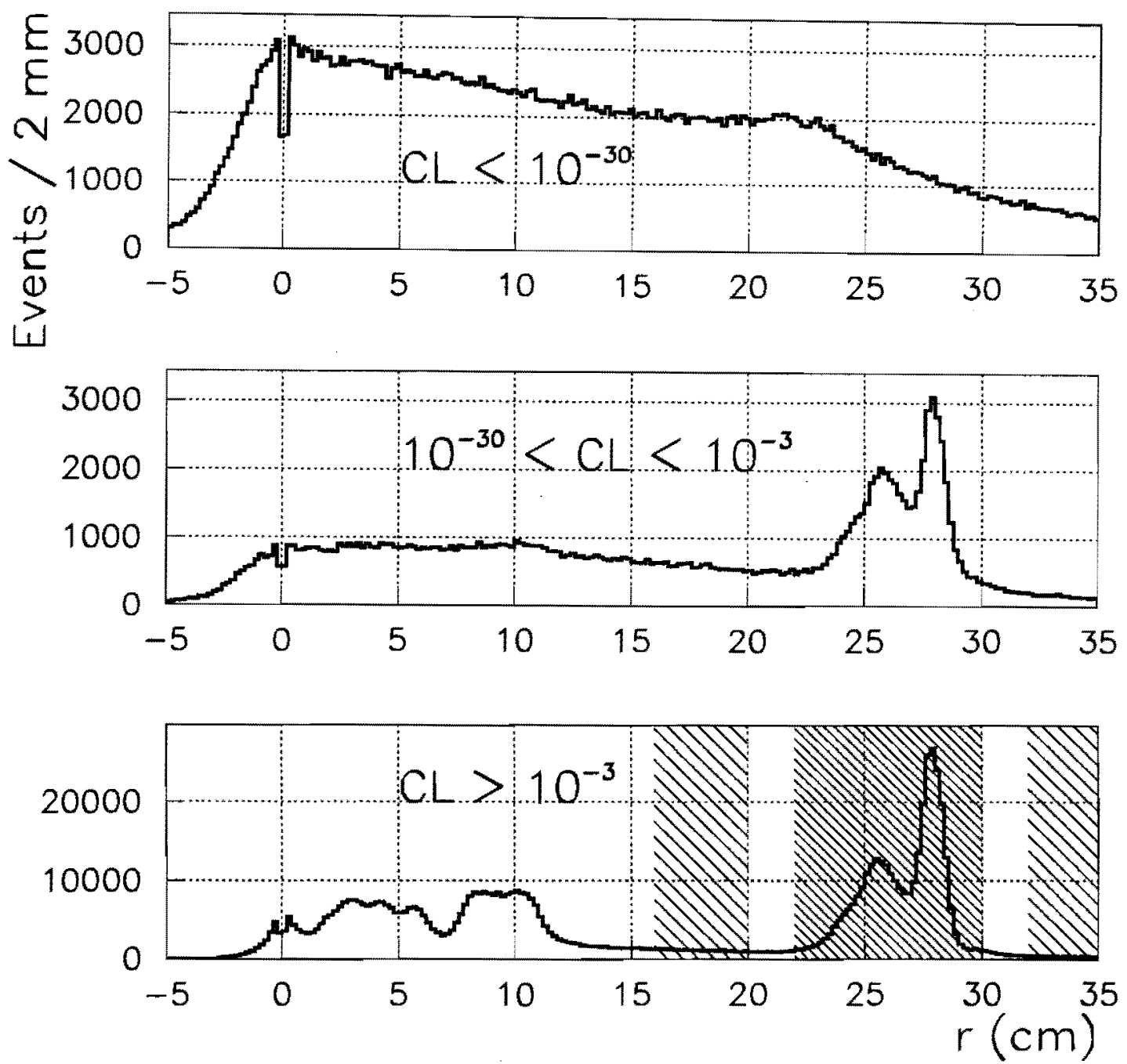

Figure 4.33: Radius distributions of the conversion vertex for the different fitting CL regions.

we check the inefficiency by using the conversion candidates themselves. We define the signal regions, $22 \mathrm{~cm}<r<30 \mathrm{~cm}$, and the side band region, $16 \mathrm{~cm}<r<20 \mathrm{~cm}$ and $32 \mathrm{~cm}<r<36 \mathrm{~cm}$. We obtain the real CL distribution by subtracting the background contribution for the CTC can conversion events by using the side band events of the radius distribution

The top plot of Figure 4.34 shows the $\log _{10}$ (CL) distribution for the signal region (solid histogram) and side band region (dashed histogram). Two histograms show good agreement at the lower CL $\left(<10^{-20}\right)$, and it ensures the background subtraction to be working very well. After integrating the background-subtracted CL distribution, we obtain the efficiency curve (bottom plot). The efficiency for the CL $>0.1 \%$ cut, which should be $99.9 \%$ by definition, is estimated to be $\sim 88 \%$.

There are no significant excesses of the conversions in the middle plot of Figure 4.33 

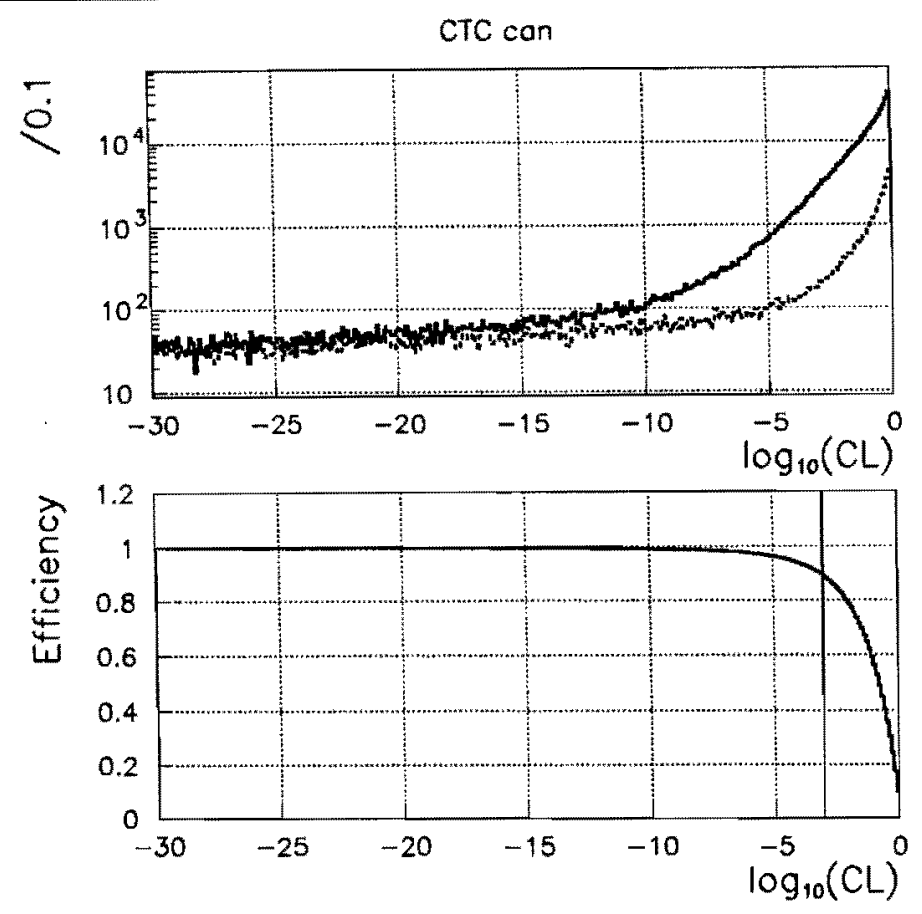

Figure 4.34: Conversion constraint fitting CL distribution in the CTC can radius signal region events (solid histogram) and side band events (dashed histogram), and the CL cut efficiency curve (bottom plot).

except the CTC can, however, by looking at the $z$ distribution in the region of $12<$ $r<21 \mathrm{~cm}$, we see clear excess at the $z=0$ which corresponds to the SVX readout cables (Figure 4.35). We apply the same background subtraction and the CL $>0.1 \%$ cut efficiency is obtained to be $\sim 95 \%$ in the bottom plot of Figure 4.36. In the SVX radius, $3<r<11 \mathrm{~cm}$, we find a clear excess at $|z|=28 \mathrm{~cm}$ which corresponds to the SVX end plate (Figure 4.37 ) and the CL $>0.1 \%$ cut efficiency is obtained to be $\sim 95 \%$ (Figure 4.38).

Figures 4.39 and 4.40 show a summary of the estimation. We see clear $r$ dependence of the CL $>0.1 \%$ cut efficiency. By weighting the efficiency according to the $r$ distribution (Figure 4.33, we find the average efficiency to be $92 \%$, where the MC sample gives $97 \%$ (Figure 4.41). On the other hand Figure 4.42 shows the efficiency curve for $J / \psi \rightarrow e e$ which is obtained by subtracting the CL distribution for the background by using the $J / \psi$ mass side band events. The CL $>0.1 \%$ cut efficiencies are estimated to be $97 \%$ and $98 \%$ for data and background, respectively.

In summary, the MC overestimates the CL cut efficiency for conversions $\left(\epsilon_{v t x}(\gamma)\right)$ and $J / \psi\left(\epsilon_{v t x}(J / \psi)\right)$ by $\sim 5 \%$ and $\sim 1 \%$, respectively. Thus the ratio $\left(\epsilon_{v t x}(\gamma) /\left(\epsilon_{v t x}(J / \psi)\right)\right.$ is overestimated $\sim 4 \%$. We, therefore, simply assign a $4 \%$ of the systematic uncertainty in the conversion vertex fit. 

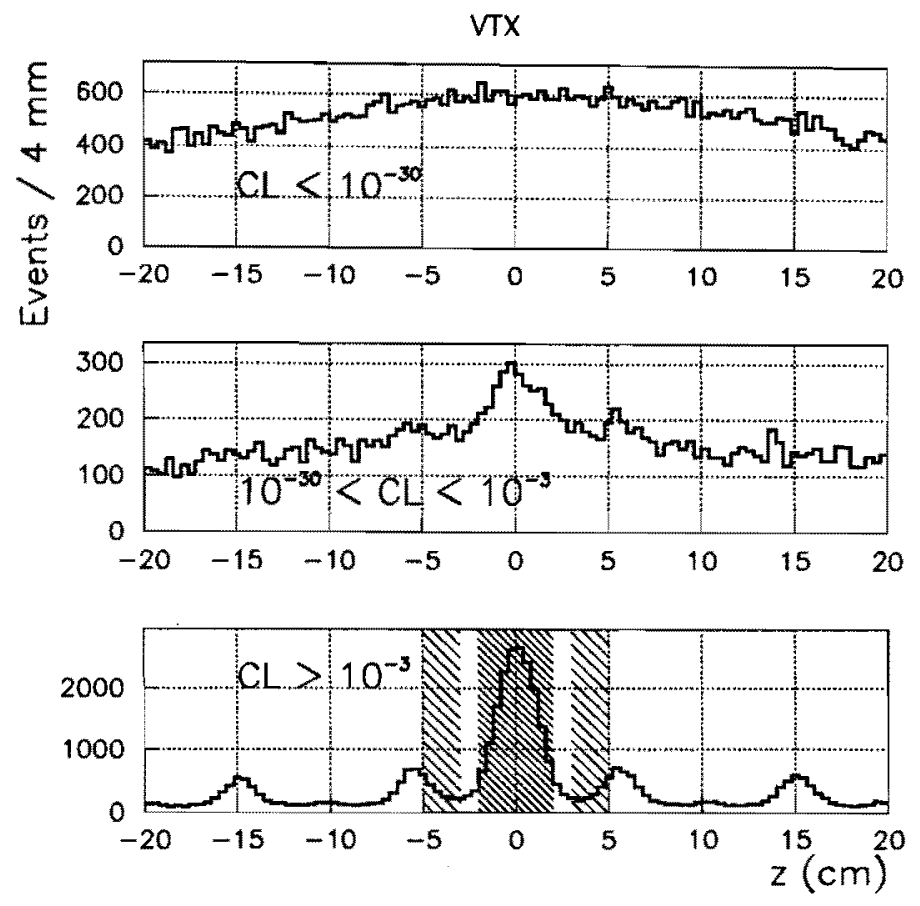

Figure 4.35: $z$ of the conversion vertex distributions in the VTX radius for the different fitting CL region.
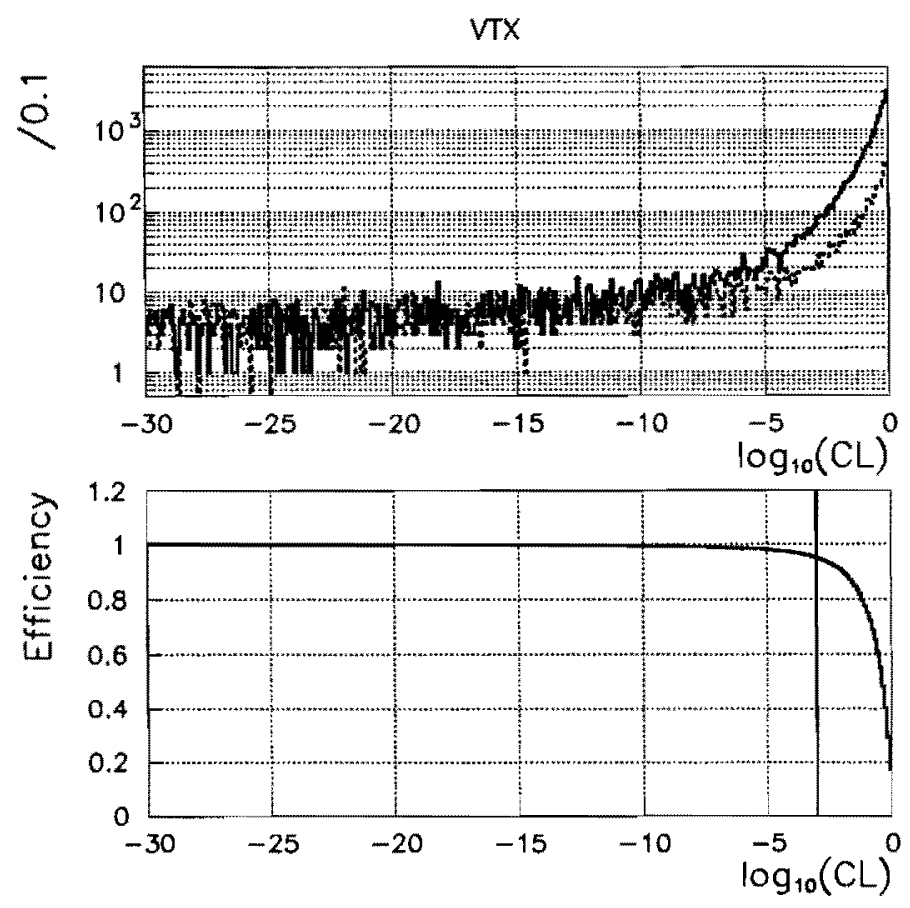

Figure 4.36: Conversion constraint fitting probability cut efficiency in the VTX radius. 

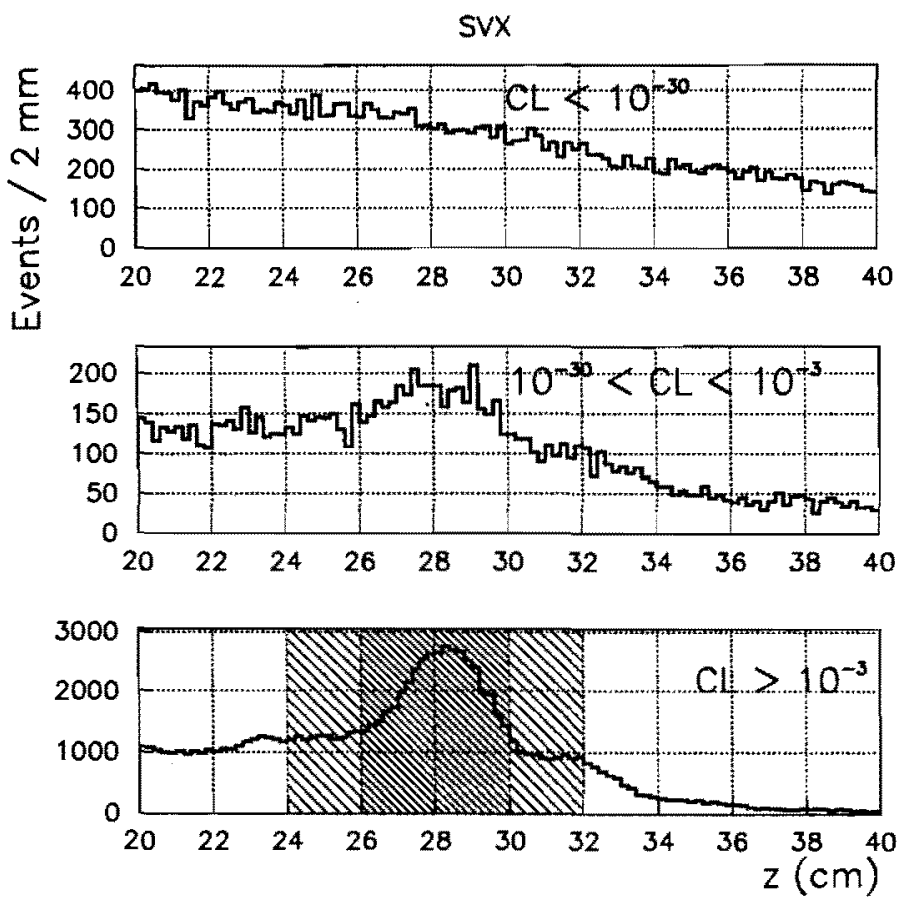

Figure 4.37: $|z|$ of the conversion vertex distributions in the SVX radius for the different fitting CL region.
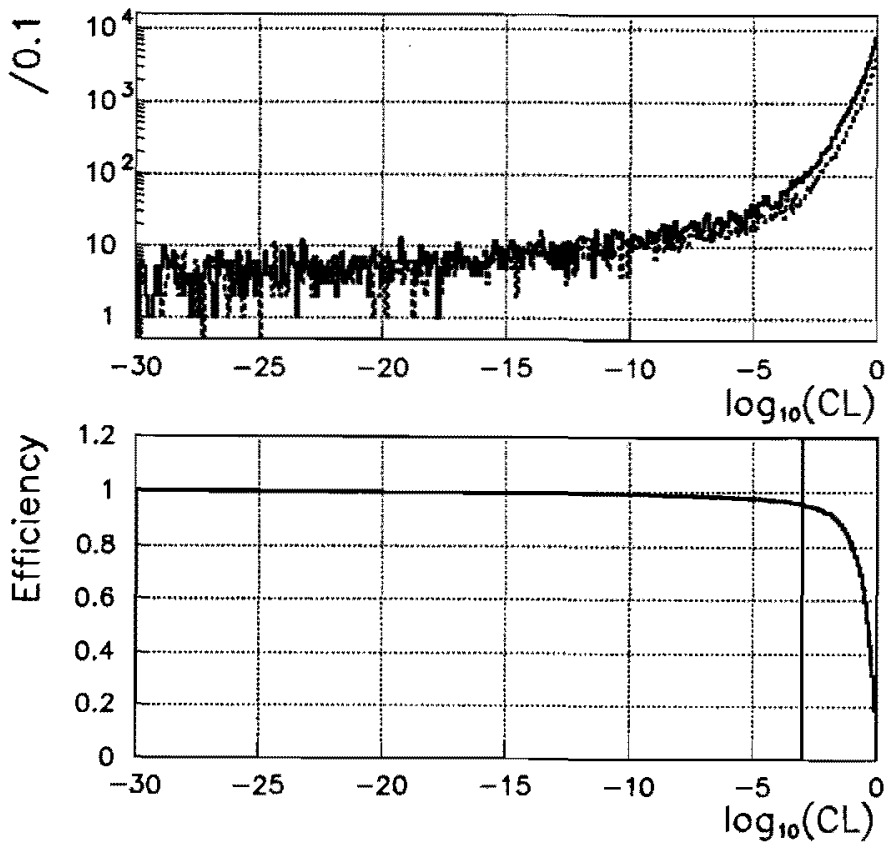

Figure 4.38: Conversion constraint fitting probability cut efficiency in the VTX radius. 


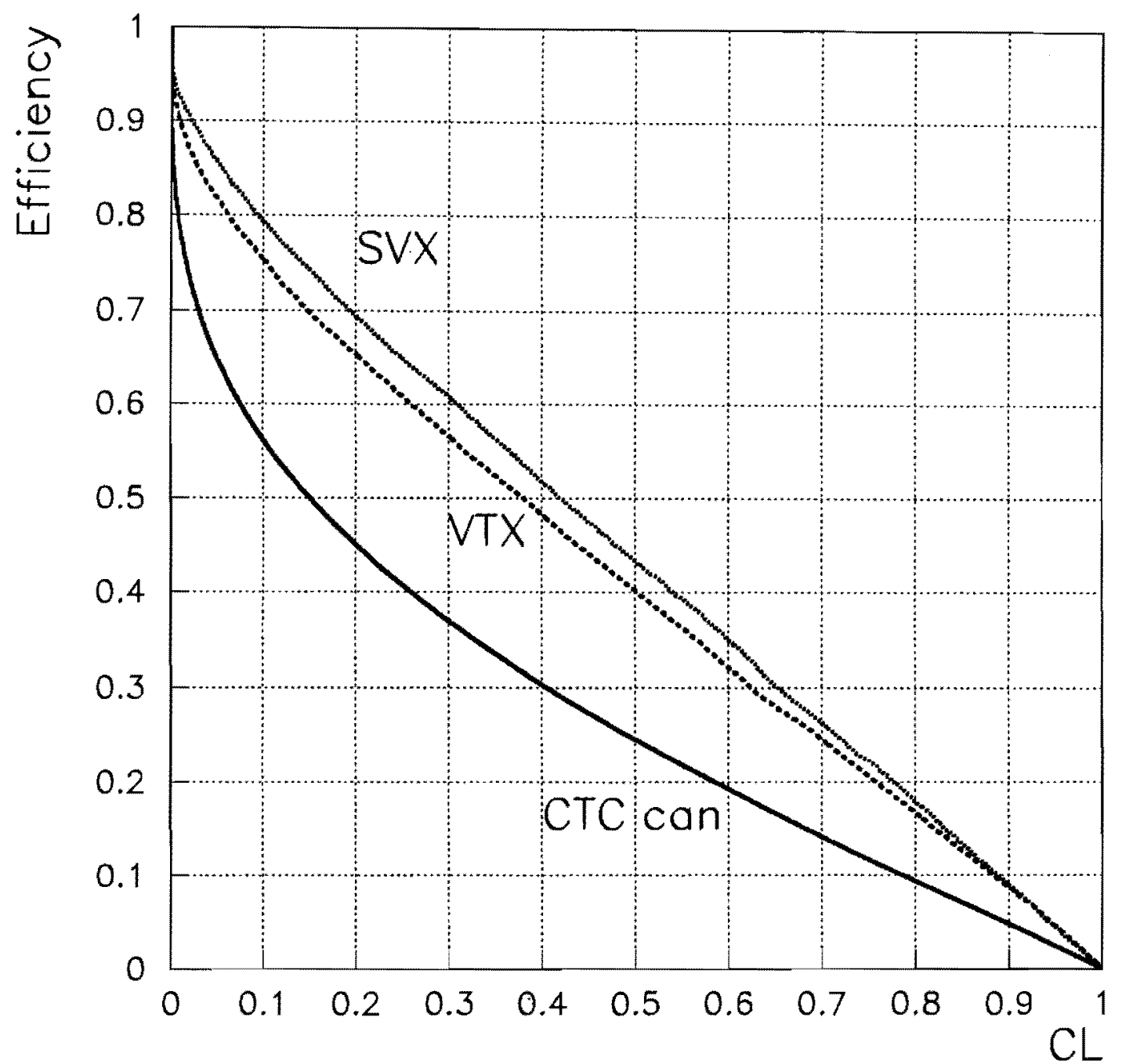

Figure 4.39: Conversion constraint fitting CL cut efficiency in the various radius regions. 


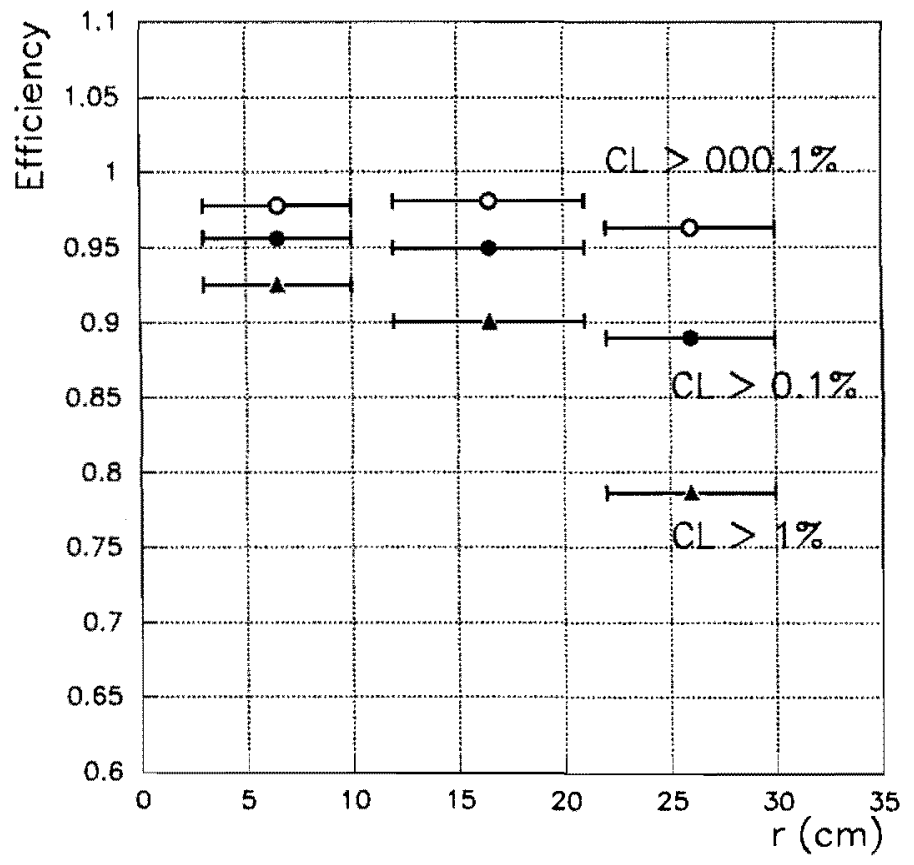

Figure 4.40: Conversion constraint fitting CL cut efficiency as a function of the vertex radius.

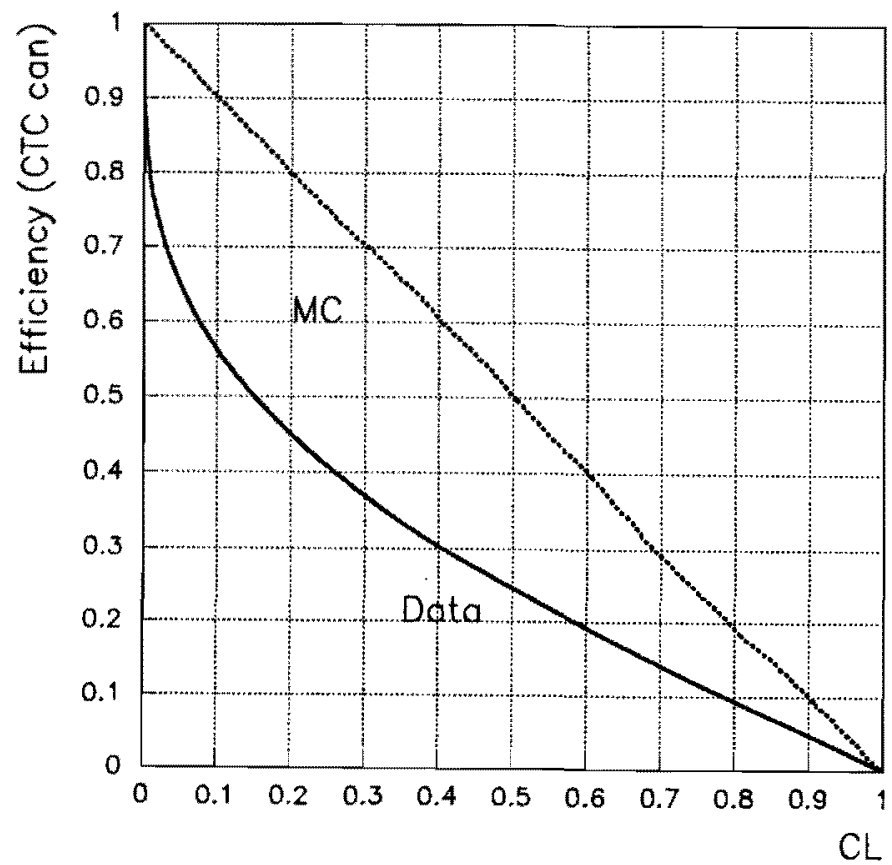

Figure 4.41: Conversion constraint fitting CL cut efficiency obtained from the data (solid line) and MC (dashed line). 


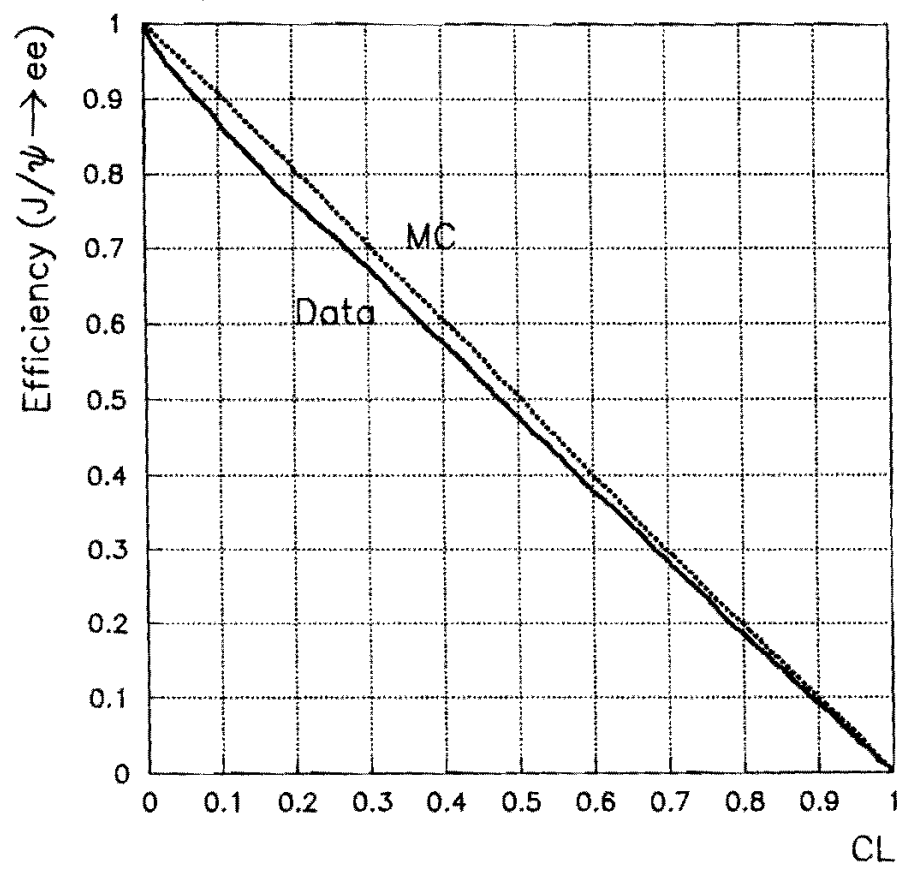

Figure 4.42: Common vertex constraint fitting CL cut efficiency for the $J / \psi \rightarrow$ $e e$ obtained from the data (solid line) and MC (dashed line).

\subsubsection{3 $\Lambda$ Vertex Constraint Fitting Efficiency}

The $\Lambda$ vertex constraint fit CL cut efficiency $\left(\left(\epsilon_{v t x}(\Lambda)\right)\right)$ can also have the same problem as the conversions. We estimate the efficiency by using the same sample in data as used for the tracking efficiency study (See Section 4.5.1.3). Figure 4.43 shows the CL distribution for the $K_{S}^{0}$ (top) and $\Lambda$ (bottom) vertex constraint fit. Figure 4.44 shows the obtained efficiency curves. Efficiencies for the sample with $r>20 \mathrm{~cm}$ are also shown in the plot and it looks there is no $r$ dependence. The efficiency the CL $>0.1 \%$ cut is estimated to be $\sim 97 \%$ and shows good agreement with that estimated from the MC sample $(\sim 98 \%)$.

\subsection{Summary of the Efficiency Estimation}

The efficiencies for the radiative $B$ signals are obtained in terms of the ratio between the reference $\bar{B}_{u} \rightarrow J / \psi K^{-}$decay. Table 4.1 shows a summary of the efficiency estimation for each of the decay modes.

The obtained efficiency ratios are $0.073,0.085$, and 0.059 for the $\bar{B}_{d}, \bar{B}_{s}$, and $\Lambda_{b}$ radiative decays, respectively. For example, the ratio for $\bar{B}_{d} \rightarrow \bar{K}^{* 0} \gamma$ is $0.064 \times$ $(0.96 / 0.75) \times 0.89$, where 0.064 is the ratio of the efficiencies estimated by using $\mathrm{MC}$ 

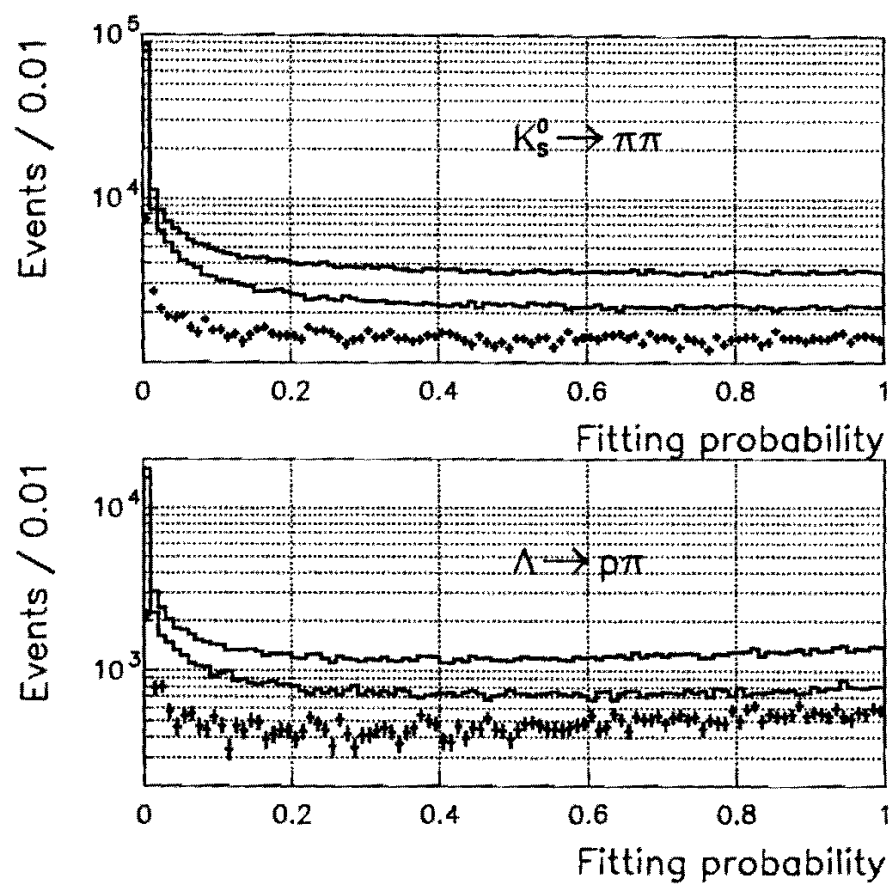

Figure 4.43: The CL distribution for the $K_{S}^{0}$ (top) and $\Lambda$ (bottom) vertex constraint fit in the strange meson mass signal and side band events (histograms). The real distributions after background subtraction are shown as points.

calculation, 0.96 is the CTC pattern recognition efficiency, 0.75 is the $J / \psi \rightarrow e e$ partner electron quality cut efficiency, and 0.89 is the scale factor for the conversion probability. The difference of the efficiencies between the radiative decays and $\bar{B}_{u} \rightarrow J / \psi K^{-}$is dominated by the conversion probability.

We define the sensitivity $(S)$ to a particular decay mode by

$$
S=\frac{\text { (branching fraction) }}{\text { (number of events) }}
$$

which can be rewritten with known quantities by using Equations (4.5)-(4.7), for example of the $\bar{B}_{d}$ channel,

$$
S\left(\bar{B}_{d} \rightarrow \bar{K}^{* 0} \gamma\right)=\frac{B\left(\bar{B}_{u} \rightarrow J / \psi K^{-}\right)}{N_{J / \psi K}} \frac{B(J / \psi \rightarrow e e)}{B\left(\bar{K}^{* 0} \rightarrow K^{-} \pi^{+}\right)}\left[\frac{f_{d}}{f_{u}} \frac{\epsilon\left(\bar{K}^{* 0} \gamma\right)}{\epsilon(J / \psi K)} \cdot\right]^{-1}
$$

It represents the branching fraction necessary to observed one event with our detector. They are $4.5 \times 10^{-5}, 9.7 \times 10^{-5}$, and $2.7 \times 10^{-4}$ for $\bar{B}_{d}, \bar{B}_{s}$, and $\Lambda_{b}$, respectively. The difference in the sensitivities between the decay modes is dominated by the difference in the fragmentation fractions. The uncertainties in the estimation of the efficiencies and the sensitivities are discussed in the next chapter. 


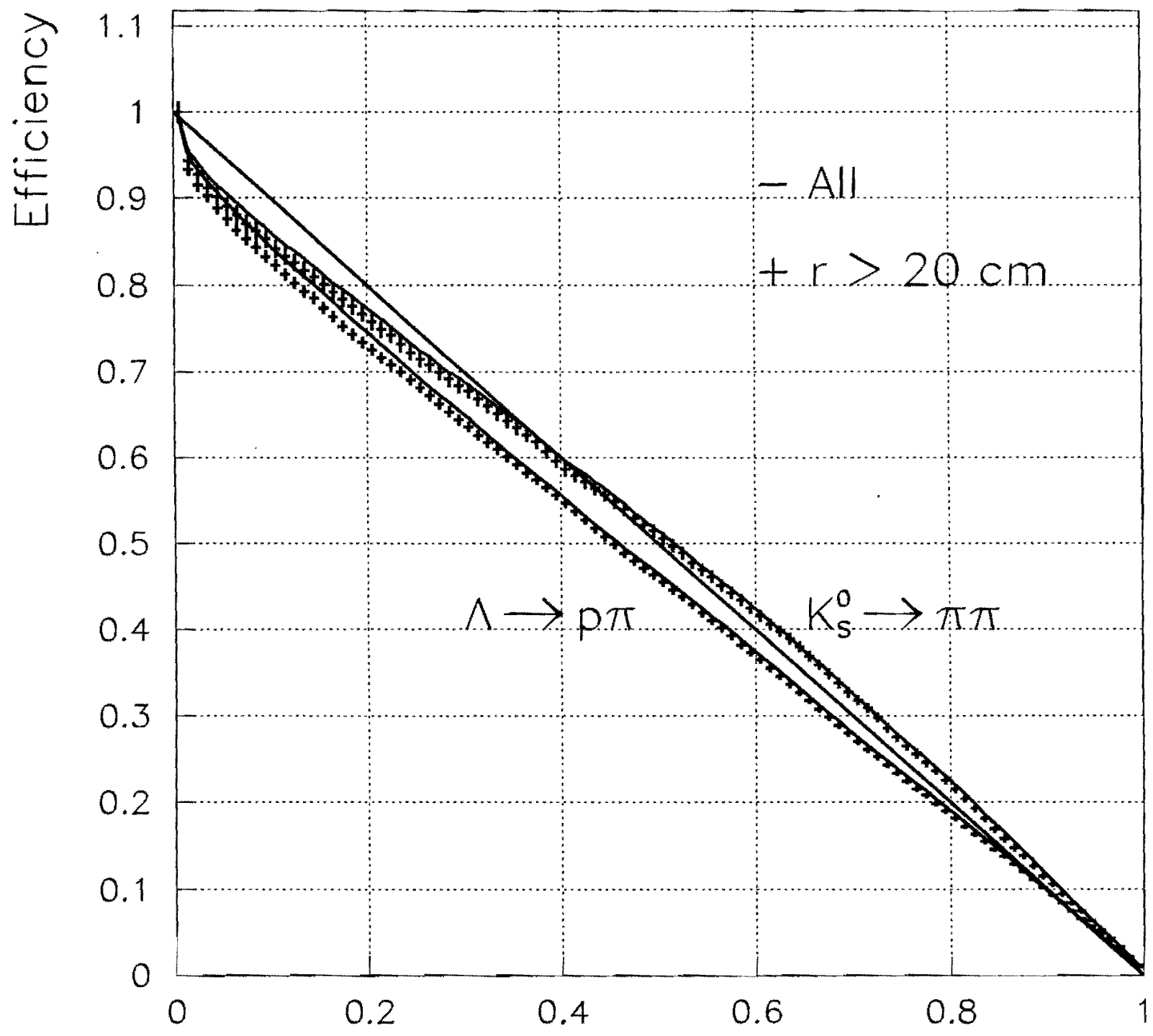

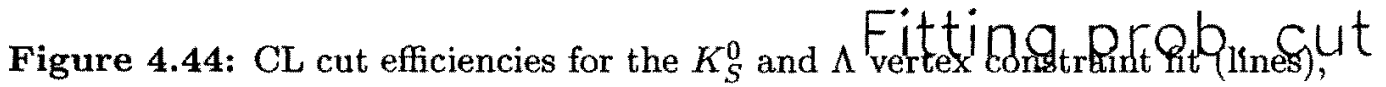
and those for the events with decay radius $>20 \mathrm{~cm}$ (points). 
Table 4.1: Ingredients for the calculation of the branching fractions of $\bar{B}_{d} \rightarrow \bar{K}^{* 0} \gamma, \bar{B}_{s} \rightarrow \phi \gamma$, and $\Lambda_{b} \rightarrow \Lambda \gamma$ decays according to Eqs. (4.5)-(4.7).

\begin{tabular}{|c|c|c|c|}
\hline & $\bar{B}_{d} \rightarrow \bar{K}^{* 0} \gamma$ & $\bar{B}_{s} \rightarrow \phi \gamma$ & $\Lambda_{b} \rightarrow \Lambda_{\gamma}$ \\
\hline$N_{\text {obs }}$ (events) & 1 & 0 & 2 \\
\hline$N_{\text {bg }}$ (events) & $0.6 \pm 0.3$ & $0.1 \pm 0.1$ & $3.4 \pm 0.6$ \\
\hline$N_{J / \psi K}$ (events) & $28.0 \pm 5.8$ & $35.0 \pm 6.4$ & $24.0 \pm 5.3$ \\
\hline$f_{d, s, \Lambda_{b}} / f_{u}$ & 1 & $0.426 \pm 0.076$ & $0.236 \pm 0.082$ \\
\hline$B\left(\bar{K}^{* 0} \rightarrow K^{-} \pi^{+}\right)$ & $2 / 3$ & - & - \\
\hline$B\left(\phi \rightarrow K^{+} K^{-}\right)$ & - & $0.491 \pm 0.008$ & - \\
\hline$B\left(\Lambda \rightarrow p \pi^{-}\right)$ & 一 & - & $0.639 \pm 0.005$ \\
\hline$B\left(\bar{B}_{u} \rightarrow J / \psi K^{-}\right)$ & \multicolumn{3}{|c|}{$(0.99 \pm 0.10) \times 10^{-3}$} \\
\hline$B(J / \psi \rightarrow e e)$ & \multicolumn{3}{|c|}{$(6.02 \pm 0.19) \times 10^{-2}$} \\
\hline CTC tracking & \multicolumn{3}{|c|}{$0.960 \pm 0.020$} \\
\hline$J / \psi$ partner electron & \multicolumn{3}{|c|}{$0.749 \pm 0.028$} \\
\hline$\Lambda$ quality cut & - & - & $0.721 \pm 0.018$ \\
\hline$X_{T}(\mathrm{DATA}) / X_{T}(\mathrm{MC})$ & \multicolumn{2}{|c|}{$0.889 \pm 0.052$} & $0.954 \pm 0.047$ \\
\hline$\left[\epsilon_{\text {signal }} / \epsilon_{J / \psi K}\right]_{M C}$ & 0.0644 & 0.0748 & 0.0666 \\
\hline$\epsilon_{\text {signal }} / \epsilon_{J / \psi K}$ & 0.0733 & 0.0853 & 0.0588 \\
\hline Sensitivity & $4.36 \times 10^{-5}$ & $9.54 \times 10^{-5}$ & $2.80 \times 10^{-4}$ \\
\hline
\end{tabular}




\section{Chapter 5}

\section{Systematic Uncertainties}

One of the dominant systematic uncertainties of this analysis is due to the statistical uncertainty of the $J / \psi K$ measurement, which contributes $21 \%, 18 \%$, and $22 \%$ for the $\bar{B}_{d}, \bar{B}_{s}$, and $\Lambda_{b}$ channels, respectively as shown in Table 4.1 . The systematic uncertainties due to size of the Monte Carlo sample are $4 \%, 3 \%$, and $4 \%$ for $\bar{B}_{d}, \bar{B}_{s}$, and $\Lambda_{b}$, respectively. The systematics due to the branching fractions are dominated by that of $B\left(\bar{B}_{u} \rightarrow J / \psi K^{-}\right)$, and we assign $11 \%$ for all the decay modes.

\subsection{Class 1 Systematics}

The systematic uncertainties for the class 1 efficiencies are not canceled in the efficiency ratios, and we explicitly estimate them. The conversion probability depends on the material thickness of the CDF detector. We assign a $5 \%(5 \%)$ systematic uncertainty originating from the transverse material thickness determination of $6.23 \pm 0.31 \% X_{0}$ $\left(6.60 \pm 0.34 \% X_{0}\right)$ for $\bar{B}_{d} \rightarrow \bar{K}^{* 0} \gamma$ and $\bar{B}_{s} \rightarrow \phi \gamma\left(\Lambda_{b} \rightarrow \Lambda \gamma\right)$. We assign a $4 \%$ systematic uncertainty for the quality cut efficiency for the partner electron of $J / \psi \rightarrow e e$ and a $1 \%$ systematic uncertainty for the $d E / d x$ cut efficiency for the $\Lambda \rightarrow p \pi$. These two systematics are caused by the statistics of the data for the efficiency estimation. We assign a $2 \%$ systematic uncertainty for the CTC tracking efficiency for $p_{T}>400 \mathrm{MeV} / c$. This uncertainty is caused by the instantaneous luminosity and electric charge dependence.

\subsection{Class 2 Systematics}

Most of the systematic uncertainties for the class 2 efficiencies are expected to be canceled in the efficiency ratios. The remaining systematics are due to the differences between the radiative and $\bar{B}_{u} \rightarrow J / \psi K^{-}$decays. We already discussed the $\mathrm{Had} / E M$ cut efficiency for the trigger electron (Section 4.3.2.2) and conversion constraint cut efficiency (Section 4.5.3.2), and assigned $5 \%$ and $4 \%$ uncertainties, respectively. Here we discuss 
the uncertainties of the $B$-hadron $p_{T}$ distribution

\subsection{1 $B$-Badron $p_{T}$ Distribution}

The uncertainty of the $B$-hadron $p_{T}$ distribution originates from the uncertainties in the $b$-quark $p_{T}$ distribution and the fragmentation model. Since the $b$-quark $p_{T}$ distributions are the same between the $B$-hadron species, the uncertainties are expected to be correlated, thus greatly reduced in the efficiency ratios between the radiative $B$ decays and the $\bar{B}_{u} \rightarrow J / \psi K^{-}$decays. We confirm this by using Monte Carlo samples with different input parameters: $m_{b}=4.5,4.75$, and $5.0 \mathrm{GeV} / c^{2} ; \mu=0.5 \mu_{0}, \mu_{0}$, and $2 \mu_{0}$. Individual efficiencies for the radiative and $\bar{B}_{u} \rightarrow J / \psi K^{-}$decays change by $\sim 20 \%$, but the efficiency ratios are, as expected, stable within the uncertainties due to the finite Monte Carlo sample sizes. As for the $B$-hadron $p_{T}$, we assume the same spectra for the $\bar{B}_{u}$ and $\bar{B}_{d}$ mesons, whereas the other $B$-hadron $p_{T}$ distributions are not necessarily the same because of the effects from the hadronization processes. This effect is checked by changing the Peterson parameter by one standard deviation away from the central value: $\epsilon_{b}=0.006 \pm 0.002[42]$.

Before doing that, however, we have checked a possible the correlation between the efficiencies in our analysis and the fragmentation fractions taken from [40]. In that analysis, the fragmentation fractions $f_{s} / f_{u}$ and $f_{\Lambda_{b}} / f_{u}$ were obtained by measuring the observed numbers of $\bar{B}_{s} \rightarrow e^{-} D_{s} X$ and $\Lambda_{b} \rightarrow e^{-} \Lambda_{c} X$ decays, respectively, normalized to the number of $\bar{B}_{u} \rightarrow e^{-} D^{0} X$ decays. For example, $f_{s} / f_{u}$ is obtained from:

$$
\frac{N_{e D_{s}}}{N_{e D^{0}}} \propto \frac{f_{s}}{f_{u}} \times \frac{\epsilon_{e D s}}{\epsilon_{e D^{0}}}
$$

where $\epsilon_{e D^{0}}$ and $\epsilon_{e D_{s}}$ are detection efficiencies for the $\bar{B}_{u} \rightarrow e^{-} D^{0} X$ and $\bar{B}_{s} \rightarrow e^{-} D_{s} X$ channels, respectively. Then our measurement is actually described by

$$
\frac{N_{\phi \gamma}}{N_{J / \psi K}} \propto \frac{N_{e D_{s}}}{N_{e D^{0}}} \times \frac{\epsilon_{\phi \gamma} / \epsilon_{e D_{s}}}{\epsilon_{J / \psi K} / \epsilon_{e D^{0}}}
$$

While the individual efficiencies of the radiative decays, the $\bar{B}_{u} \rightarrow J / \psi K^{-}$. decays, and the semileptonic decays changes by $\sim 7 \%$ when the Peterson parameter is varied, the efficiency ratios, $\epsilon_{\phi \gamma} / \epsilon_{e D_{s}}, \epsilon_{\Lambda \gamma} / \epsilon_{e \Lambda_{c}}$, and $\epsilon_{J / \psi K} / \epsilon_{e D^{0}}$, are found to be stable within the Monte Carlo statistical uncertainties. We thus consider that the systematic uncertainties of the $B$-hadron $p_{T}$ distributions cancel. The quoted $18 \%(35 \%)$ total uncertainty of the $f_{s} / f_{u}\left(f_{\Lambda_{b}} / f_{u}\right)$ value includes a $6 \%(6 \%)$ systematic uncertainty originating from the Peterson parameter. By subtracting it, we reassign the resulting $17 \%(34 \%)$ to the systematic uncertainty of $f_{s} / f_{u}\left(f_{\Lambda_{b}} / f_{u}\right)$. 
Table 5.1: Summary of the systematic uncertainties.

\begin{tabular}{lccc} 
& $\bar{B}_{d} \rightarrow \bar{K}^{* 0} \gamma$ & $\bar{B}_{s} \rightarrow \phi \gamma$ & $\Lambda_{b} \rightarrow \Lambda \gamma$ \\
\hline$J / \psi K$ statistics & $21 \%$ & $18 \%$ & $22 \%$ \\
MC statistics & $4 \%$ & $3 \%$ & $4 \%$ \\
\hline Class 1 efficiency & & & \\
Conversion probability & $6 \%$ & $6 \%$ & $5 \%$ \\
$J / \psi$ partner electron & $4 \%$ & $4 \%$ & $4 \%$ \\
$\Lambda d E / d x$ & - & - & $3 \%$ \\
CTC pattern recognition & $2 \%$ & $2 \%$ & $2 \%$ \\
\hline Class 2 efficiency & & & \\
$H a d / E M$ & $5 \%$ & $5 \%$ & $5 \%$ \\
Conversion vertex fit & $4 \%$ & $4 \%$ & $4 \%$ \\
\hline Input physics parameters & & & \\
Fragmentation fractions & $0 \%$ & $17 \%$ & $34 \%$ \\
Branching fractions & $11 \%$ & $11 \%$ & $11 \%$ \\
\hline Total & $26 \%$ & $29 \%$ & $43 \%$
\end{tabular}

\subsection{Class 3 Systematics}

The systematics uncertainties for the class 3 efficiencies are exactly canceled in the efficiency ratios by definition.

\subsection{Summary of the Systematics}

Table 5.1 shows a summary of the systematic uncertainty estimation for each decay modes. The total systematic uncertainty in measurering the branching fraction for the radiative $\bar{B}_{d}, \bar{B}_{s}$, and $\Lambda_{b}$ decays is estimated to be $25 \%, 27 \%$, and $43 \%$, respectively. 


\section{Chapter 6}

\section{Limit}

Since there are small backgrounds for the $\bar{B}_{d}$ and $\bar{B}_{s}$ radiative decays, we set upper limits on the branching fractions without background subtraction.

We calculate an upper limit $\left(N_{\mathrm{CL}}\right)$ on the mean number of radiative decays at a given CL, including the total systematic uncertainty $\left(\sigma_{\mathrm{syst}}\right)$, by numerically solving the following equation for $N_{\mathrm{CL}}$ :

$$
1-\mathrm{CL}=\sum_{n=0}^{N_{\text {obs }}} \mathcal{P}_{N_{\mathrm{CL},}, \sigma_{\mathrm{syst}}}(n),
$$

where $N_{\text {obs }}$ is the number of candidates, and $\mathcal{P}_{\mu, \sigma}(n)$ is defined with $P_{\mu}(n)$ (Poisson distribution) and $G_{\mu, \sigma}(x)$ (Gaussian distribution) as follows:

$$
\mathcal{P}_{\mu, \sigma}(n)=\int_{0}^{\infty} P_{x}(n) G_{\mu, \sigma}(x) d x .
$$

Figure 6.1 shows the $N_{\mathrm{CL}}$ as a function of the systematic uncertainty at the $90 \%$ (top) and $95 \%$ (bottom) confidence levels.

For the $\Lambda_{b}$ case, we set an upper limit after subtracting the backgrounds by using a toy Monte Carlo which generates the numbers of signal and background events in a trial according to the probability distributions $\mathcal{P}_{N_{\mathrm{CL}}, \sigma_{\text {gyst }}}(n)$ and $\mathcal{P}_{N_{\mathrm{bg}}, \sigma_{\mathrm{bg}}}(n)$, respectively, where $\mathcal{P}_{\mu, \sigma}(n)$ is defined in Eq. (6.2), $N_{\mathrm{CL}}$ is the upper limit on the number of decays for a given CL, $\sigma_{\text {syst }}$ is the systematic uncertainty on the signal yield, $N_{\mathrm{bg}}$ is the number of backgrounds, with uncertainty $\sigma_{\mathrm{bg}}$. The CL is given by the fraction of the trials which has the total number of signal and background events exceeding the observed number of events, but still has the fewer background events than the observed number of events. Fibure 6.2 shows the CL as a function of the $N_{C L}$ for the $\Lambda_{b}$ analysis: $N_{o b s}=2 ; 43 \%$ systematic uncertainty; and $N_{b g}=3.4 \pm 0.6$ events.

We obtain $N_{90 \%}=4.3,2.6$, and 4.5 and $N_{95 \%}=5.5,3.5$, and 6.8 , for the $\bar{B}_{d}$, $\bar{B}_{s}$, and $\Lambda_{b}$ decays, respectively. The upper limits on the branching fraction is written by $B r_{\mathrm{CL}}=S \times N_{\mathrm{CL}}$. Finally we get upper limits on the branching fractions for the $\bar{B}_{d} \rightarrow \bar{K}^{* 0} \gamma, \bar{B}_{s} \rightarrow \phi \gamma$, and $\Lambda_{b} \rightarrow \Lambda \gamma$ decays to be $1.9 \times 10^{-4}, 2.5 \times 10^{-4}$, and $1.3 \times 10^{-3}$ at the $90 \% \mathrm{CL}$, and $2.4 \times 10^{-4}, 3.4 \times 10^{-4}$, and $1.9 \times 10^{-3}$ at the $95 \% \mathrm{CL}$, respectively. 

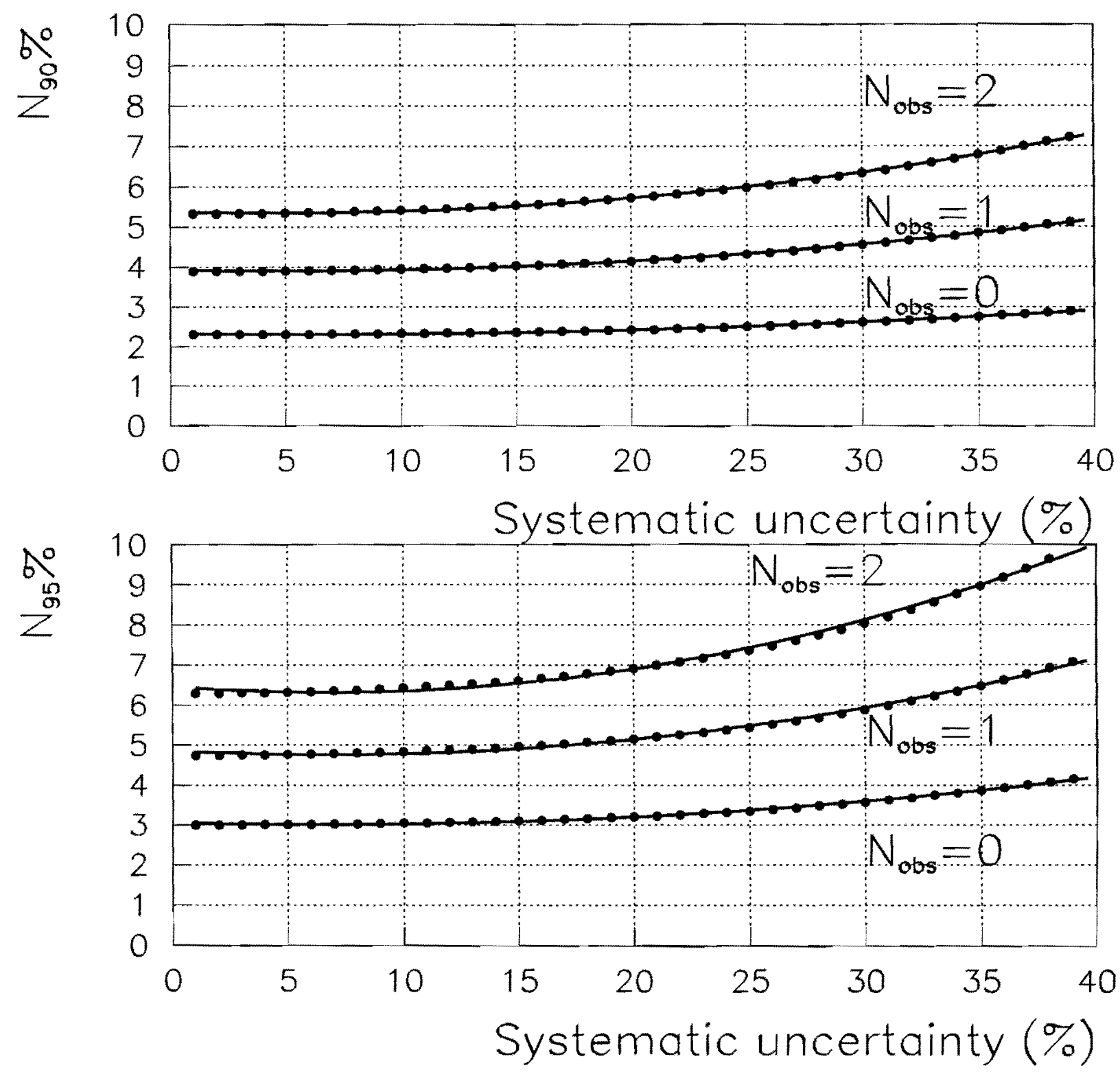

Figure 6.1: $N_{90 \%}$ and $N_{95 \%}$ as a function of the systematic uncertainty. 


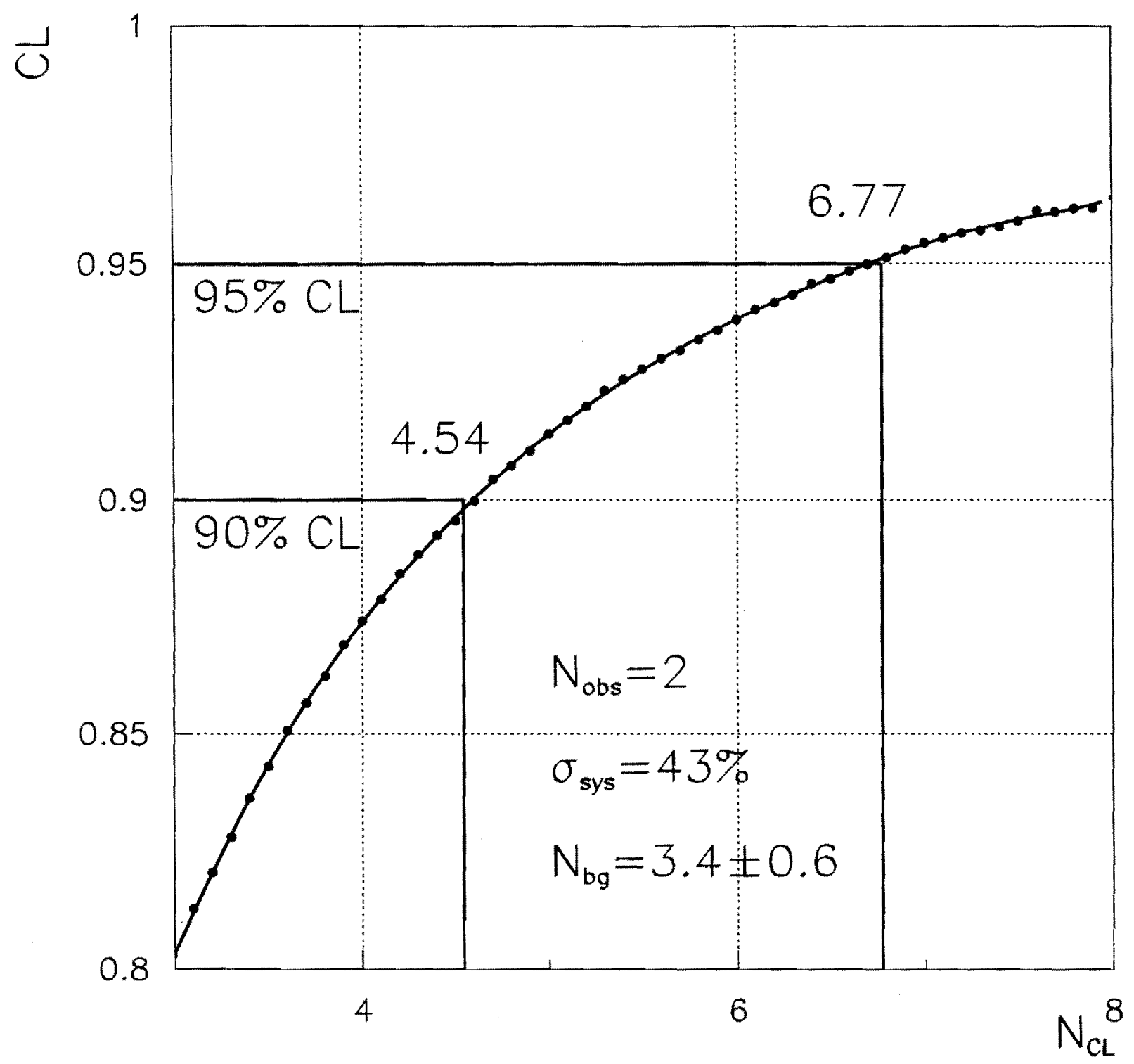

Figure 6.2: $N_{90 \%}$ and $N_{95 \%}$ with $N_{o b s}=2, N_{b g}=3.4 \pm 0.6$, and $\sigma_{s y s}=43 \%$. 


\section{Chapter 7}

\section{Conclusions}

We searched for $\bar{B}_{d} \rightarrow \bar{K}^{* 0}\left(\rightarrow K^{-} \pi^{+}\right) \gamma, \bar{B}_{s} \rightarrow \phi\left(\rightarrow K^{+} K^{-}\right) \gamma, \Lambda_{b} \rightarrow \Lambda\left(\rightarrow p \pi^{-}\right) \gamma$, and their charge conjugate decays, in $74 \mathrm{pb}^{-1}$ of data resulting from $p \bar{p}$ collisions at $\sqrt{s}=1.8$ $\mathrm{TeV}$ recorded by $\mathrm{CDF}$.

In this analysis, the photon was identified by an electron-positron pair produced through the external photon conversion before the tracking detector volume. One of the conversion electrons with $E_{T}>8 \mathrm{GeV}$ served as a trigger for event recording, where no additional tracks coming from the strange hadron decays were required. There were two merits for using the photon conversions: 1) the reconstructed $B$ mass resolution with the photon conversions is better those with the calorimeter photons; 2) the fitting with the conversion constraint reduced the combinatorial backgrounds.

We optimized the selection cuts by maximizing the figure of merit defined by $\epsilon_{\text {sig }} / \sqrt{\epsilon_{\mathrm{bg}}}$, where $\epsilon_{\text {sig }}$ and $\epsilon_{\mathrm{bg}}$ were, respectively, the efficiency for the signal and background events. After all the selection cuts, there were 1,0 , and 2 events of the $\bar{B}_{d}, \bar{B}_{s}$, and $\Lambda_{b}$ candidates with expected backgrounds of $0.6 \pm 0.3,0.1 \pm 0.1$, and $3.3 \pm 0.6$ events.

Since we did not find significant excesses over background for all the radiative decay modes, we set uppers limits on the branching fractions in the following chapters. We inferred the branching fractions of radiative $B$ decays by forming ratios with the known branching fraction $\bar{B}\left(\bar{B}_{u} \rightarrow J / \psi K^{-}\right)$, where the $J / \psi$ was reconstructed as $J / \psi \rightarrow$ $e e$ decays. The topology observed in this "reference" channel was similar to the one resulting from the radiative decay. This way, any common factors canceled in the ratio of branching fractions, for example, the $b$ quark production cross section, and systematic uncertainties were reduced.

We obtained upper limits on the branching fractions for the $\bar{B}_{d} \rightarrow \bar{K}^{* 0} \gamma, \bar{B}_{s} \rightarrow \phi \gamma$, and $\Lambda_{b} \rightarrow \Lambda \gamma$ decays to be

$$
\begin{aligned}
B\left(\bar{B}_{d} \rightarrow \bar{K}^{* 0} \gamma\right) & <1.9 \times 10^{-4} \\
B\left(\bar{B}_{s} \rightarrow \phi \gamma\right) & <2.5 \times 10^{-4}, \\
B\left(\Lambda_{b} \rightarrow \Lambda \gamma\right) & <1.3 \times 10^{-3}
\end{aligned}
$$


at the $90 \% \mathrm{CL}$, and

$$
\begin{aligned}
B\left(\bar{B}_{d} \rightarrow \bar{K}^{* 0} \gamma\right) & <2.4 \times 10^{-4}, \\
B\left(\bar{B}_{s} \rightarrow \phi \gamma\right) & <3.4 \times 10^{-4}, \\
B\left(\Lambda_{b} \rightarrow \Lambda \gamma\right) & <1.9 \times 10^{-3},
\end{aligned}
$$

at the $95 \%$ CL.

The limit on the $\bar{B}_{d}$ channel is consistent with the measuerment by CLEO, $B\left(B^{0, \pm} \rightarrow\right.$ $\left.K^{*} \gamma\right)=\left(4.55_{-0.68}^{+0.72} \pm 0.34\right) \times 10^{-5}$. The limit of the $\bar{B}_{s}$ channel is better than the results of the DELPHI, $B\left(B_{s} \rightarrow \phi \gamma\right)<7.0 \times 10^{-4}$ at the $90 \% \mathrm{CL}$. The limit on the $\Lambda_{b}$ channel is the first experimental result. 


\section{Appendix A}

\section{Material Calibration}

\section{A.1 Biases to Radius Distribution}

If there is radius dependence for the conversion detection efficiency, we can not estimate the correct value of the $\left\langle X_{T}\right\rangle$, however some of the dependence can be canceled in the ratio between the data and $M C$ which we exactly want to know. At first we consider the sources which can bias the radius distribution. We expect three types of such biases:

- A. Conversion partner electron

The $E_{T}$ obtained by the calorimeter is contributed by three elements: (1) trigger electron; (2) partner electron; (3) underlying particles. Thus the $\gamma$ conversions with both the electrons to be in the same wedge have a higher efficiency for level 2 $\left(E_{T}>8 \mathrm{GeV}\right)$ requirements than the $\gamma$ conversions with the same momentum but with two electrons in different wedges. The two conversion electrons are parallel at the conversion vertex and the separation of the electrons after arriving the calorimeter is just caused by the magnetic bending of the electrons. Thus the fraction of the same wedge conversion is higher for the outer radius conversions. That is, the radius distribution is biased by the partner electron, and the material thickness is underestimated. However this is not a problem in comparing the $\mathrm{MC}$ and the data because our MC includes the L2 trigger simulation.

- B. $\pi_{0}$ partner photon

There is another type of bias which we expect to be different between the MC and the data. Figure A.1 shows the trigger electron $p_{T}$ distributions with two conversion electrons in the same or different wedges. The fraction of the same wedge events is also shown. The MC conversions with $p_{T}<6 \mathrm{GeV}$ are dominated by the same wedge events, while data conversions are not. Most of the conversions in the data are originated from $\pi^{0}, \rho \rightarrow \gamma \gamma$ (presence of another $\gamma$ ), and the $E_{T}$ cut efficiency for conversions in the data is higher than that for the MC samples. 


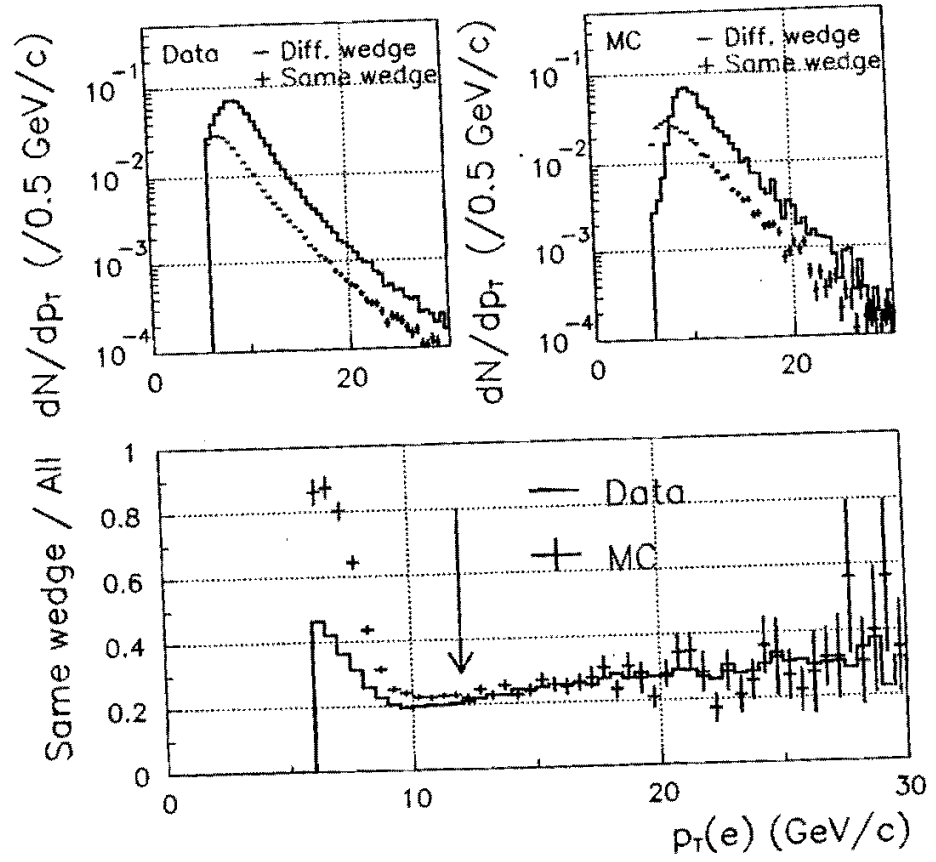

Figure A.1: $p_{T}$ distribution for the trigger $\gamma$ conversion candidates in the Run IB data and MC.

It makes the efficiency higher for the different wedge events and biases the radius distribution. Thus we require $p_{T}>12 \mathrm{GeV} / c$, where the same wedge event fraction is consistent between the $\mathrm{MC}$ and the data.

- C. Conversion constraint fit

The error matrix of the CTC track which obtained from the offline tracking code is actually not understood very well, and it is tend to be underestimated it. Thus $\chi^{2}$ of the vertexing fit such as the conversion constraint fit is usually overestimated. To avoid the problem, we usually scale the track error matrix by a factor of 2.4. By applying this correction, we obtained a reasonable $\chi^{2}$ distribution for the vertexing fit around the primary vertex. However the error for the largely displaced track from the primary vertex is still underestimated, and the fitting confidence level cut $(>0.1 \%)$ efficiency depends on the radius of the conversion.

\section{A.2 Counting the Conversion Rates}

We first subtract the Dalitz decay background in the data by using the conversion candidates with $r<0$ (Figure A.2).

Figure A.3 shows the radius distribution of the conversion vertex for the data and 


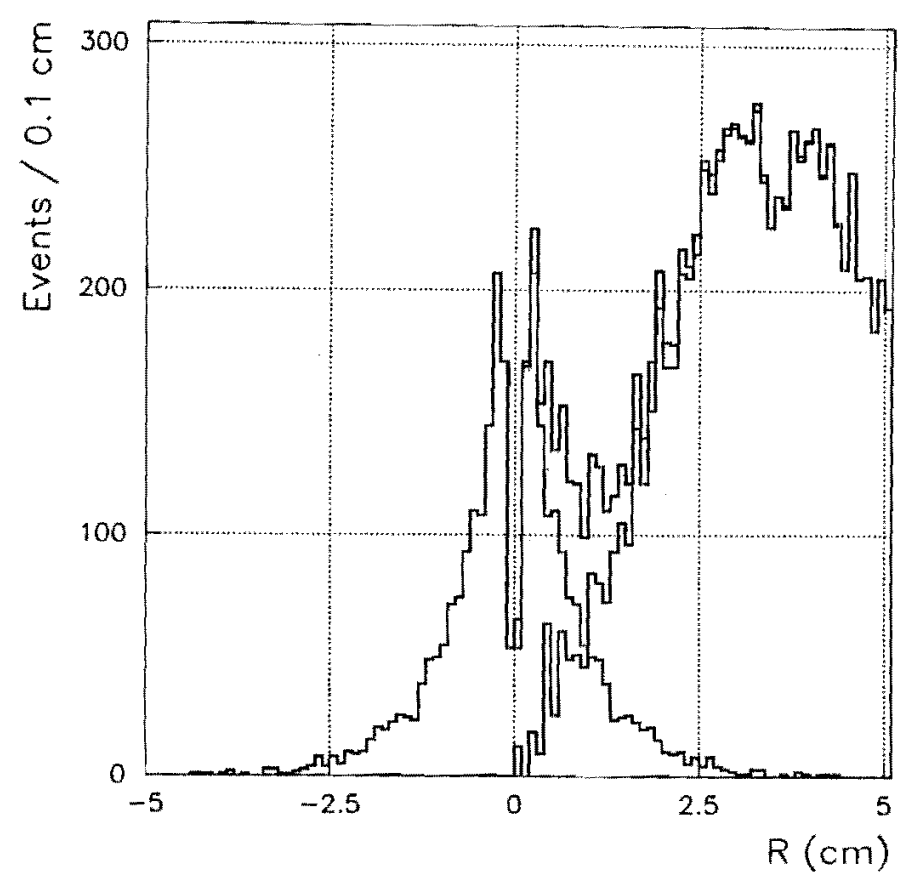

Figure A.2: Dalitz event subtraction.

MC. A peak around $r=28 \mathrm{~cm}$ for the data corresponds to the CTC can. A peak around $r=26 \mathrm{~cm}$ corresponds to the VTX outer wall. We expect contributions of the SVX cables between the VTX and the CTC, but there is no clear peak because of the R resolution $(\sim 0.5 \mathrm{~cm})$.

The radius distribution of the MC in Figure A.3 shows only one peak around $r=$ $25 \mathrm{~cm}$, because the MC simulates the photon conversion in the CTC can $\left(1.4 \% X_{0}\right)$, the SVX cable $\left(0.208 \% X_{0}\right)$, and the VTX outer wall $\left(1.81 \% X_{0}\right)$ at a common radius $(\mathrm{R}=$ $25 \mathrm{~cm}$ ), and there is no way to distinguish them. Thus we estimate the total material thickness for the MC by scaling the conversions in the peak $\left(X_{T}=3.418 \% X_{0}\right)$.

We estimate the "real" $r$ distribution of the CTC can conversions by subtracting the SVX cable contribution. We use $\phi$ of the conversion vertex. Figure A.4 shows the $\phi$ distributions for three different $R$ ranges: (1) $27.8<R<28.7 \mathrm{~cm}$ (CTC can); (2) 26.8 $<\mathrm{R}<27.8 \mathrm{~cm}$ (SVX cable); (3) $24.5<\mathrm{R}<26.8 \mathrm{~cm}$ (VTX outer wall ). Figure A.5 is the same as (2) and we see clear peaks caused by the SVX cable. We define the SVX cable region $(C)$ as shown by the dashed lines in the plot, $-136^{\circ}<\phi<-134^{\circ}$, $-132^{\circ}<\phi<-120^{\circ},-46^{\circ}<\phi<-44^{\circ},-42^{\circ}<\phi<-30^{\circ}, 46^{\circ}<\phi<58^{\circ}$, and $137^{\circ}<\phi<149^{\circ}$, where the sum of the SVX cable region corresponds $52^{\circ}$ in $\phi$.

Figure A. 6 shows the $r$ distribution for the SVX cable region, $C$, (top-left histogram) and for the outside of the region, $\bar{C}$, (top-right histogram). The "real" $r$ distribution of the SVX cable can be obtained by subtracting the remaining CTC and VTX con- 

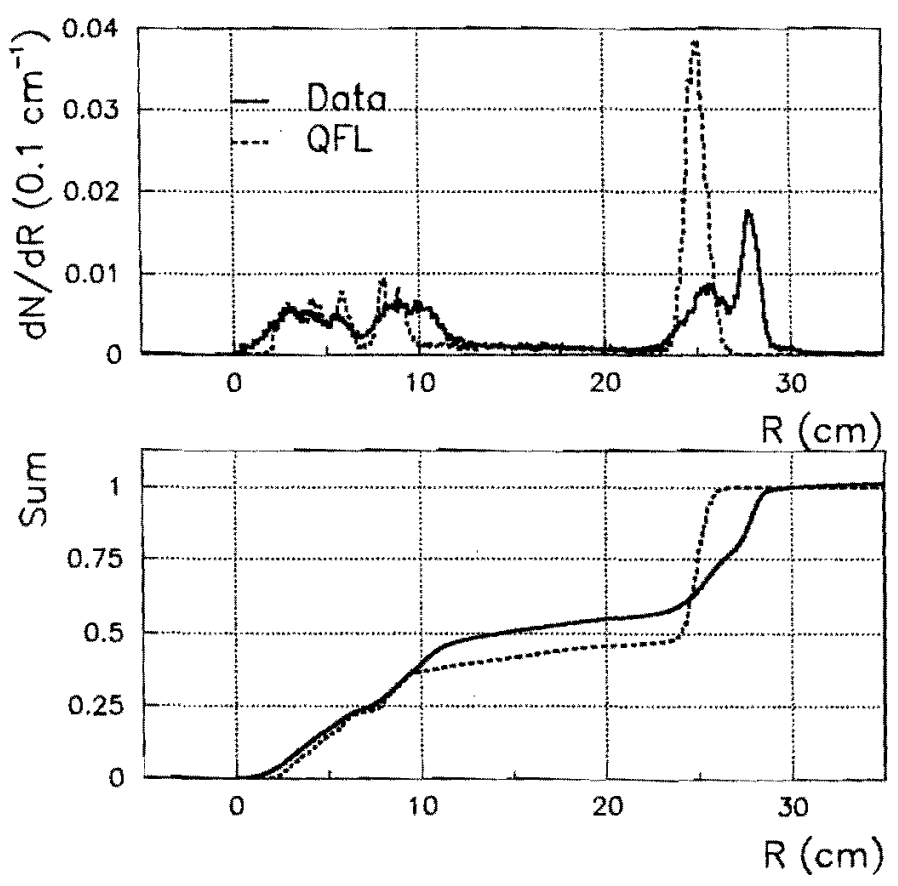

Figure A.3: Distribution of the conversion vertex radius.

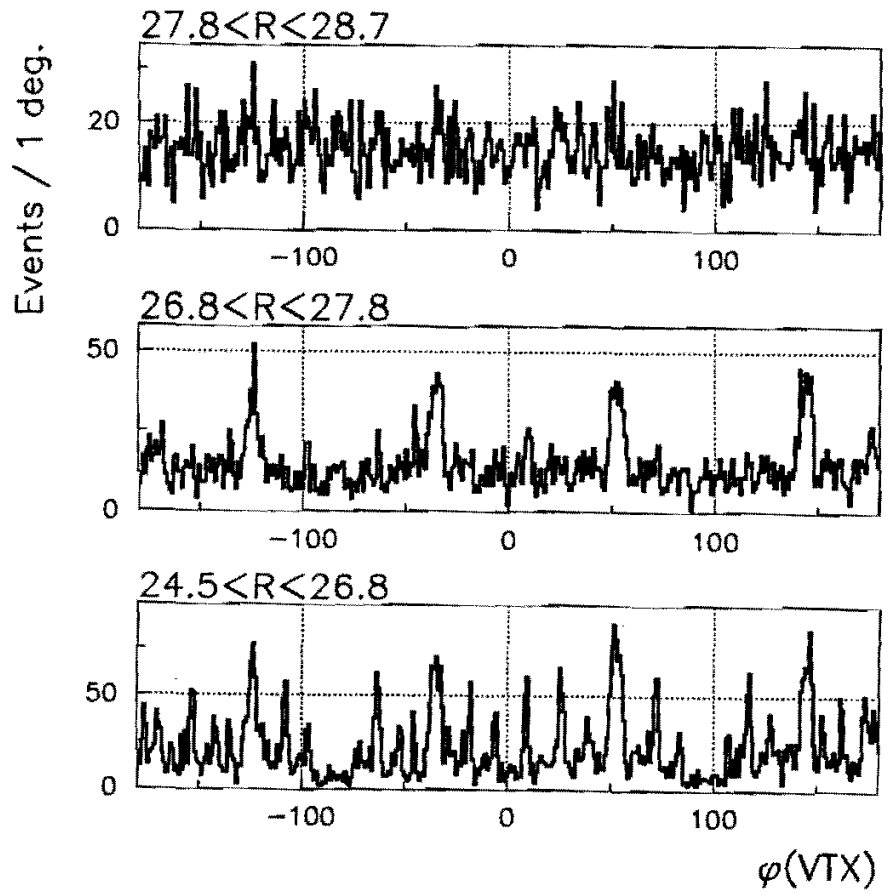

Figure A.4: $\phi$ distribution of the conversion vertex. 

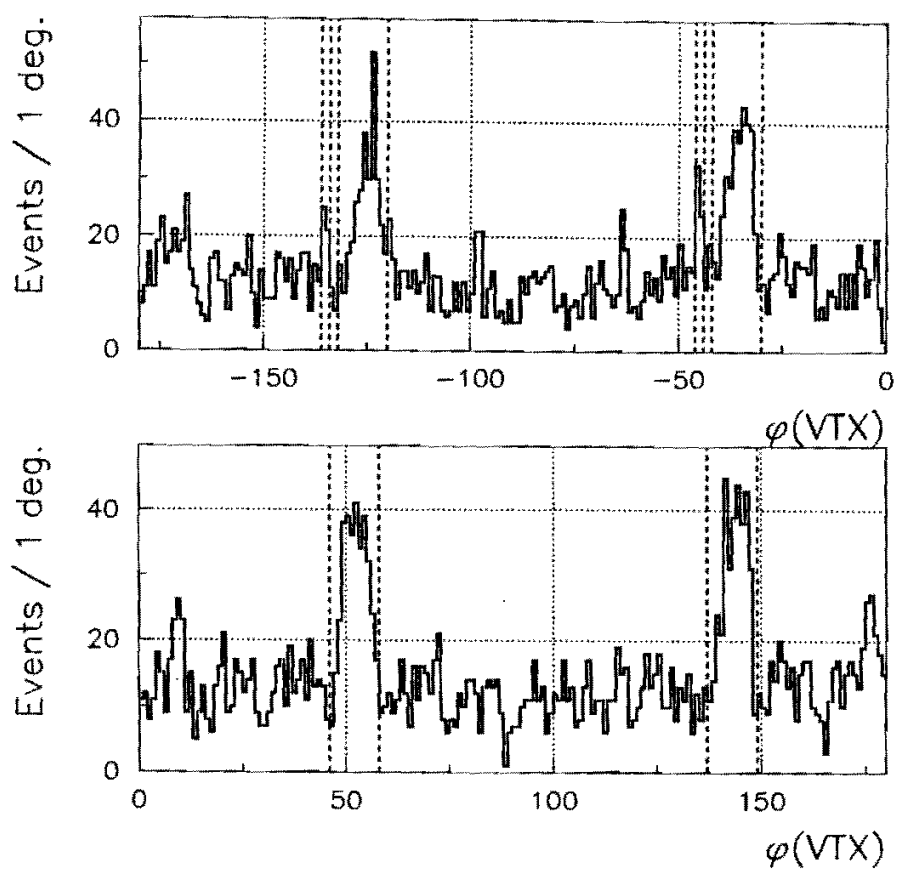

Figure A.5: $\phi$ distribution of the conversion vertex around SVX cable.

tributions which are assumed to be the same as that for the outside of the SVX cable region,

$$
r(\mathrm{SVX} \text { cable })=r(C)-\frac{52}{360-52} \times r(\bar{C})
$$

The obtained real SVX cable $r$ distribution is shown in Figure A.6 (top points). Then we obtain the real CTC can $r$ distribution by subtracting the real SVX cable $r$ distribution from that outside of the SVX cable region

$$
r(\mathrm{CTC} \text { can })=r(\bar{C})-r(\text { SVXcable })
$$

The bottom plot of Figure A.6 shows the result and the number of events in this peak is obtained by fitting the distribution to a Gaussian. Efficiency for the SVX cable removal $(\bar{C})$ is estimated by using the CTC can region events.

\section{A.3 Results}

Finally we obtain the $N(\mathrm{CTC})$ and the material thickness is calculated to be $X_{T}(\mathrm{All})=$ $(6.30 \pm 0.31) \% X_{0}$, where the uncertainty is quadratic sum of the statistics and the systematic uncertainty caused by the error of the CTC can thickness estimation $((1.26 \pm$ $\left.0.06) \% X_{0}\right)$. 

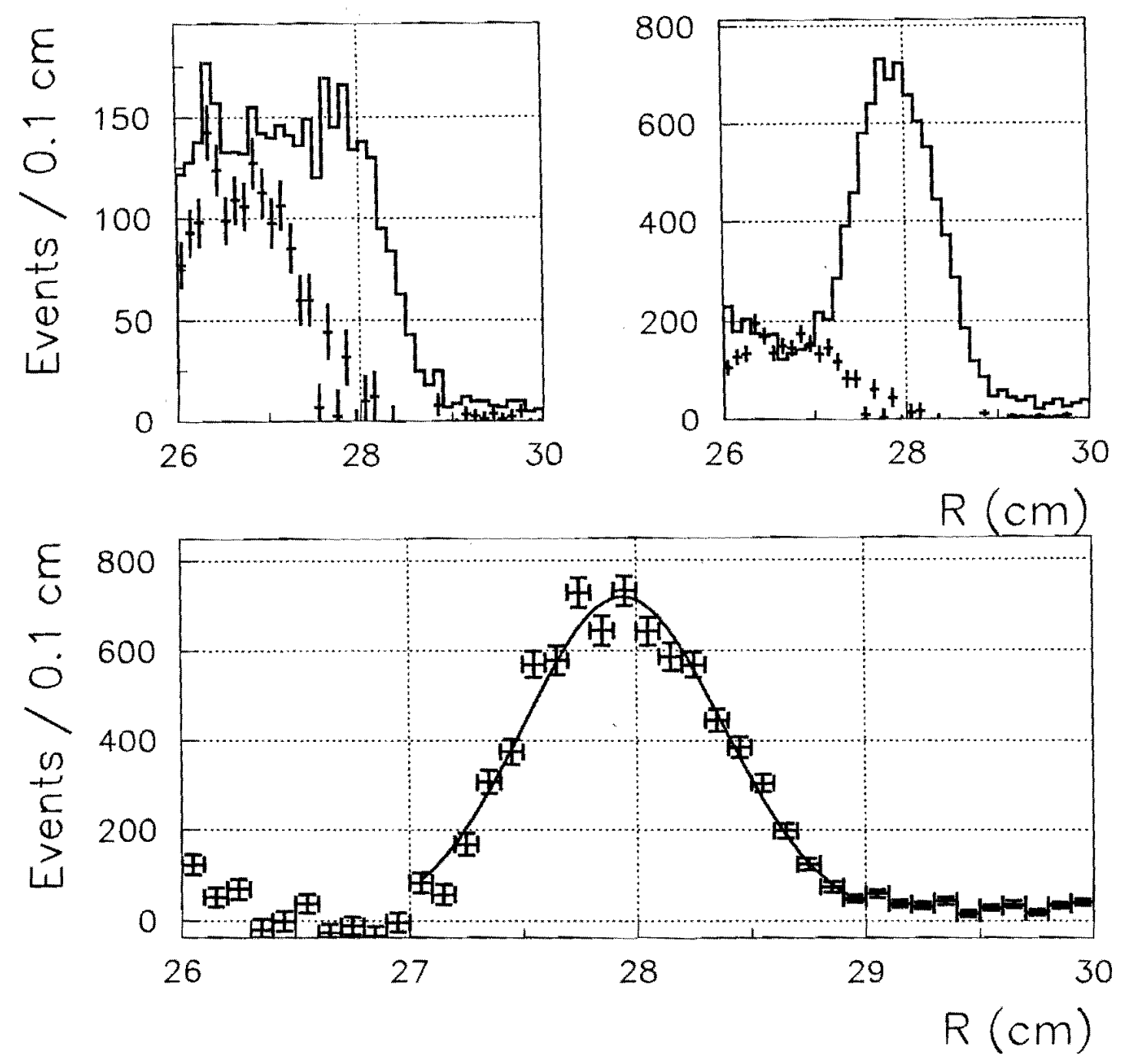

Figure A.6: Distribution of the conversion vertex radius around the CTC can for the SVX cable region (top-left histogram), for the outside the SVX cable region (top-right histogram). The "real" $R$ distributions for the SVX cable (top points) and CTC can (bottom) are also shown. 
For the MC samples, we just fit the $\mathrm{R}$ distribution without any background subtraction, and obtain $X_{T}(\mathrm{r}<30 \mathrm{~cm})=(6.53 \pm 0.10) \% X_{0}$. The MC has thicker materials than the data by $4 \%$, but they are consistent within the uncertainty.

We do the same analysis for the conversion +2 SVX sample, and obtain $X_{T}(\mathrm{R}<30 \mathrm{~cm})=$ $(6.69 \pm 0.37) \% X_{0}$ for the data and $X_{T}(\mathrm{R}<30 \mathrm{~cm})=(7.46 \pm 0.19) \% X_{0}$ for the MC sample. The MC has thicker materials than the data by $12 \%(2 \sigma)$.

\section{A.4 Vertex $\chi^{2}$ Cut Dependence}

As we discussed before, the conversion vertex $r$ distribution of the data dependeds on the vertex fitting $\chi^{2}$ probability cut. Since the MC smears the track parameter by using the error matrix itself, the MC does not show this tendency. Anyway what we want to know is the material thickness without the $\chi^{2}$ probability cut. Thus we estimate the material thickness with different $\chi^{2}$ probability cuts and extrapolate the points to zero. Figure A.7 and A.8 show the results for the conversion sample and the conversion +2 SVX sample, respectively. The points are well fitted to a function of constant + $\sqrt{\chi^{2} \text { prob. }}$ Table A.1 summarizes the results.

Table A.1: Results for material thickness estimation.

\begin{tabular}{l|lll}
\hline & Data $\left(\% X_{0}\right)$ & MC $\left(\% X_{0}\right)$ & Data/MC \\
\hline Conversion & $6.23 \pm 0.07 \pm 0.30$ & $6.53 \pm 0.07$ & $0.954 \pm 0.015 \pm 0.045$ \\
\hline Conversion + 2 SVX & $6.60 \pm 0.15 \pm 0.30$ & $7.43 \pm 0.15$ & $0.889 \pm 0.027 \pm 0.045$ \\
\hline \hline
\end{tabular}

We obtain the inner detector material thickness scale factors $X_{T}$ (DATA)/ $X_{T}(\mathrm{MC})$ to be $0.889 \pm 0.027_{\text {stat }} \pm 0.045_{\text {sys }}$ and $0.954 \pm 0.015_{\text {stat }} \pm 0.045_{\text {sys }}$ with and without the SVX fiducial requirement, respectively. 


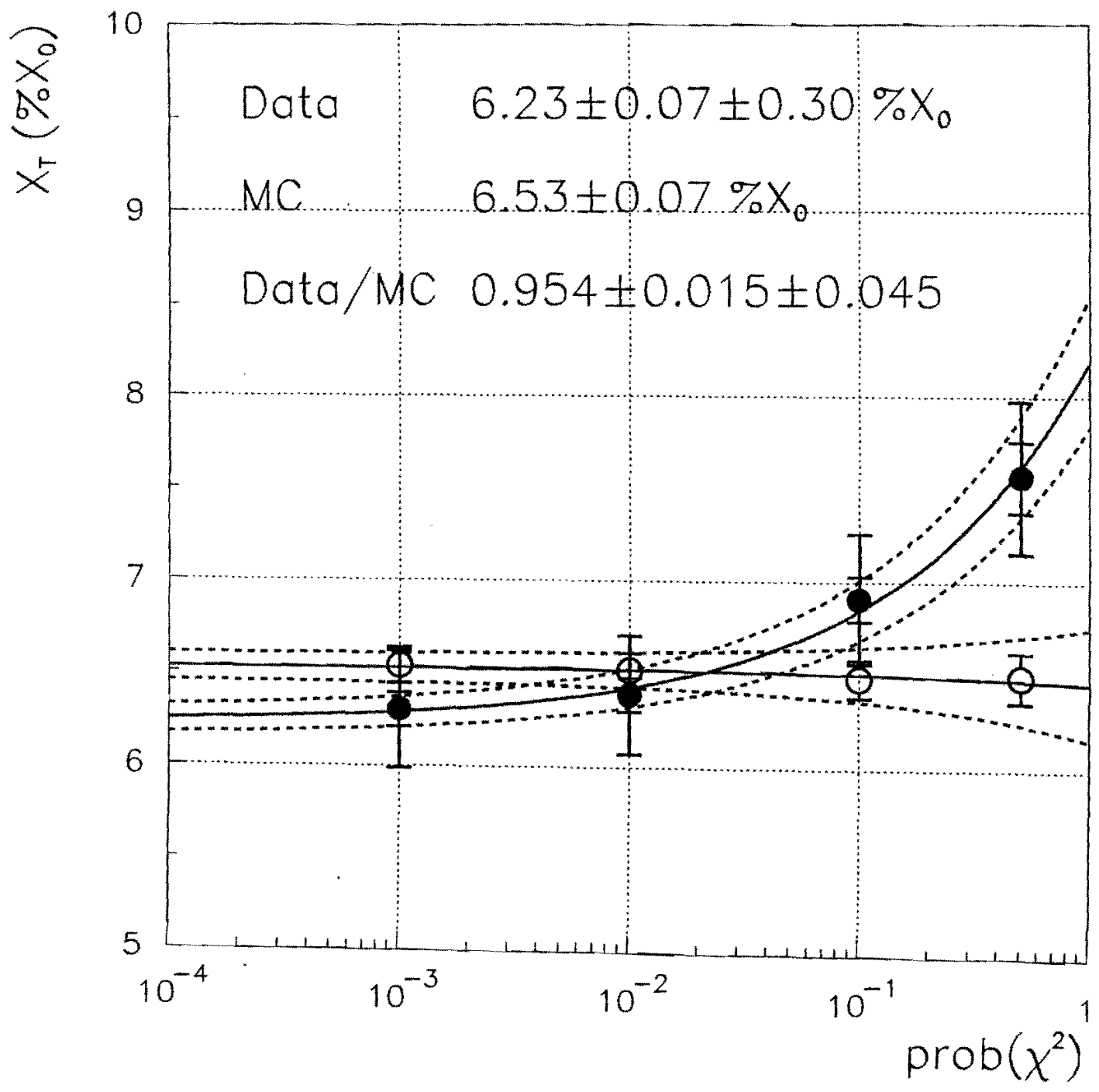

Figure A.7: Material thickness without the SVX fiducial requirement for the data (black circles) and MC (open circles). 


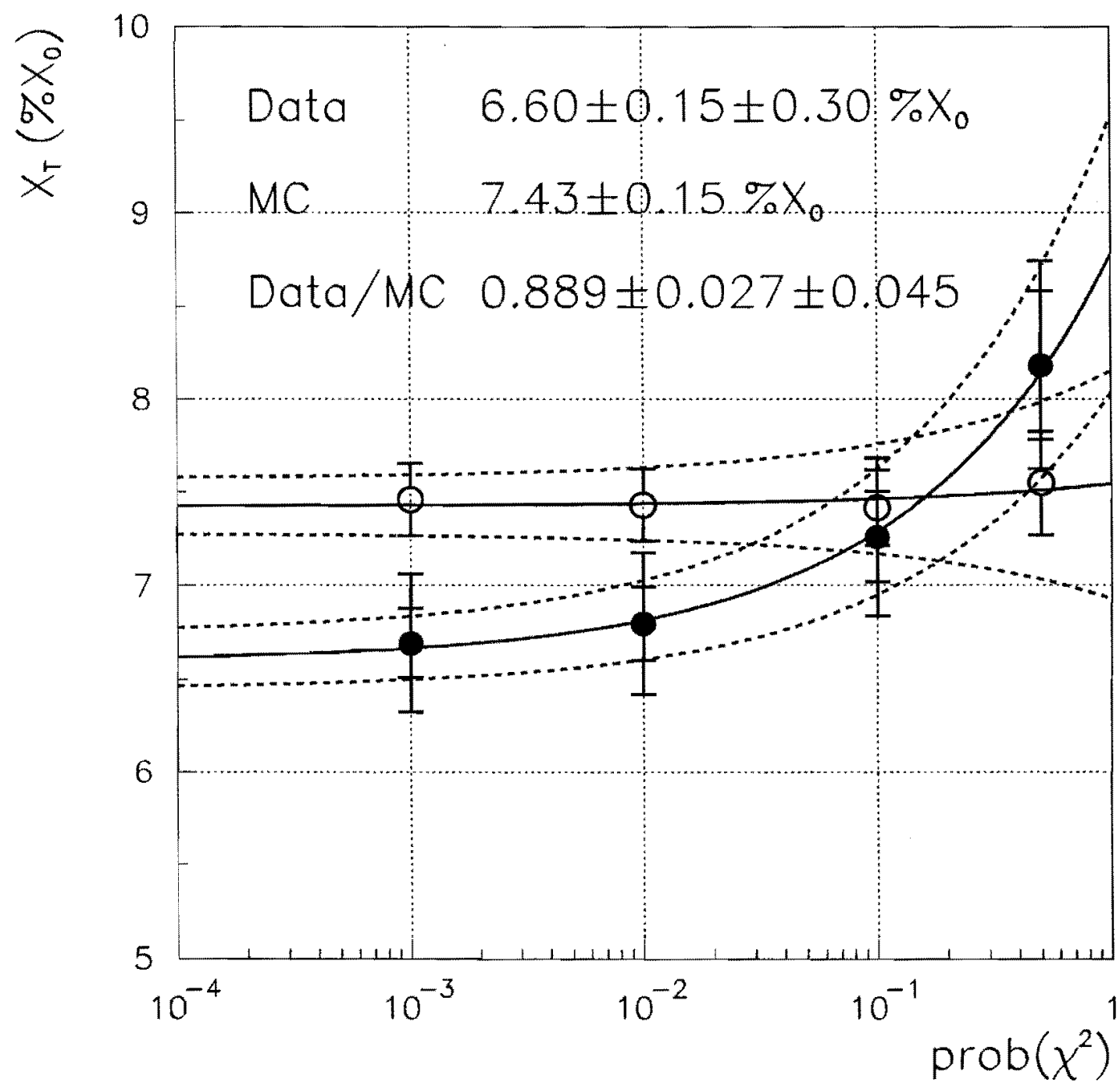

Figure A.8: Material thickness with the SVX fiducial requirement for the data (black circles) and MC (open circles). 


\section{Bibliography}

[1] S. L. Glashow, "Partial Symmetries Of Weak Interactions," Nucl. Phys. 22, 579 (1961).

[2] Y. Fukuda et al. [Super-Kamiokande Collaboration], "Evidence for oscillation of atmospheric neutrinos," Phys. Rev. Lett. 81, 1562 (1998) [hep-ex/9807003].

[3] M. Kobayashi and T. Maskawa, "CP Violation In The Renormalizable Theory Of Weak Interaction," Prog. Theor. Phys. 49, 652 (1973).

[4] N. Cabibbo, "Unitary Symmetry And Leptonic Decays," Phys. Rev. Lett. 10, 531 (1963).

[5] L. Wolfenstein, "Parametrization Of The Kobayashi-Maskawa Matrix," Phys. Rev. Lett. 51, 1945 (1983).

[6] D. E. Groom et al., "Review of particle physics," Eur. Phys. J. C15, 1 (2000).

[7] S. W. Herb et al., "Observation Of A Dimuon Resonance At $9.5 \mathrm{Gev}$ In $400 \mathrm{Gev}$ Proton - Nucleus Collisions," Phys. Rev. Lett. 39, 252 (1977).

[8] Z. Ligeti, " $\left|V_{c b}\right|$ and $\left|V_{u b}\right|$ from $B$ decays: Recent progress and limitations," hep$\mathrm{ph} / 9908432$.

[9] T. Affolder et al. [CDF Collaboration], "A measurement of $\sin 2 \beta$ from $B \rightarrow J / \psi K_{S}^{0}$ with the CDF detector," Phys. Rev. D61, 072005 (2000) [hep-ex/9909003].

[10] D. Cronin-Hennessy et al. [CLEO Collaboration], "Study of two-body B decays to kaons and pions: Observation of $B \rightarrow \pi^{+} \pi^{-}, B \rightarrow K^{ \pm} \pi^{0}$, and $B \rightarrow K^{0} \pi^{0}$ decays," hep-ex/0001010.

[11] R. Ammar et al. [CLEO Collaboration], "Evidence for penguins: First observation of $B \rightarrow K^{*}(892) \gamma$," Phys. Rev. Lett. 71, 674 (1993).

[12] T. E. Coan et al. [CLEO Collaboration], "Study of exclusive radiative $B$ meson decays," Phys. Rev. Lett. 84, 5283 (2000) [hep-ex/9912057]. 
[13] " $b \rightarrow s \gamma$ branching fraction and CP asymmetry," S. Ahmed, in Proceedings of the 4th International Symposium on Radiative Corrections (RADCOR 98): Applications of Quantum Field Theory to Phenomenology, Barcelona, Catalonia, Spain, 1998, edited by J. Sola (World Scientific, Singapore, 1999), p. 139.

[14] W. Adam et al. [DELPHI Collaboration], "Study of rare $b$ decays with the DELPHI detector at LEP," Z. Phys. C72, 207 (1996).

[15] P. A. Griffin, M. Masip and M. McGuigan, "Determination of $\left|V_{t s}\right|$ from $D \rightarrow K^{*} \ell \nu$ and $B \rightarrow K^{*} \gamma$ data via heavy quark symmetry and perturbative QCD," Phys. Rev. D50, 5751 (1994) [hep-ph/9312262].

[16] A. Ali, V. M. Braun and H. Simma, "Exclusive radiative B decays in the light cone QCD sum rule approach," Z. Phys. C63, 437 (1994) [hep-ph/9401277].

[17] R. Fleischer, "New strategies to extract beta and gamma from $B_{d} \rightarrow \pi^{+} \pi^{-}$and $B_{s} \rightarrow K^{+} K^{-}$," Phys. Lett. B459, 306 (1999) [hep-ph/9903456].

[18] J. Ellis, M. K. Gaillard, D. V. Nanopoulos and S. Rudaz, "The Phenomenology Of The Next Lefthanded Quarks," Nucl. Phys. B131, 285 (1977).

[19] A. Vainshtein, "How penguins started to fly," Int. J. Mod. Phys. A14, 4705 (1999) [hep-ph/9906263].

[20] A. J. Buras, M. Misiak, M. Munz and S. Pokorski, "Theoretical uncertainties and phenomenological aspects of $\mathrm{B} \rightarrow \mathrm{X}(\mathrm{s})$ gamma decay," Nucl. Phys. B424, 374 (1994) [hep-ph/9311345].

[21] A. J. Buras, "Weak Hamiltonian, CP violation and rare decays," hep-ph/9806471. P. F. Harrison and H. R. Quinn [BABAR Collaboration], "The BaBar physics book: Physics at an asymmetric B factory," in NONE SLAC-R-0504 Papers from Workshop on Physics at an Asymmetric B Factory (BaBar Collaboration Meeting), Rome, Italy, 11-14 Nov 1996, Princeton, NJ, 17-20 Mar 1997, Orsay, France, 16-19 Jun 1997 and Pasadena, CA, 22-24 Sep 1997.

[22] P. Singer and D. Zhang, Phys. Lett. B383, 351 (1996) [hep-ph/9606343].

[23] R. Mohanta, A. K. Giri, M. P. Khanna, M. Ishida and S. Ishida, "Weak radiative decay $\Lambda_{b} \rightarrow \Lambda \gamma$ and quark-confined effects in the covariant oscillator quark model," Prog. Theor. Phys. 102, 645 (1999) [hep-ph/9908291]. 
[24] T. Goto, Y. Okada and Y. Shimizu, "Flavor changing neutral current processes in $B$ and $K$ decays in the supergravity model," Phys. Rev. D58, 094006 (1998) [hep-ph/9804294].

[25] T. Goto, Y. Y. Keum, T. Nihei, Y. Okada and Y. Shimizu, "Effect of supersymmetric CP phases on the $B \rightarrow X_{s} \gamma$ and $B \rightarrow X_{s} \ell^{+} \ell^{-}$decays in the minimal supergravity model," Phys. Lett. B460, 333 (1999) [hep-ph/9812369].

[26] C. Chua, X. He and W. Hou, "CP violating $b \rightarrow s \gamma$ decay in supersymmetric models," Phys. Rev. D60, 014003 (1999) [hep-ph/9808431].

[27] G. W. Hou, "Large and fun CP violation in B meson decays: Where to search?," hep-ph/9902382.

[28] P. Nason, S. Dawson, and R. K. Ellis, "The One Particle Inclusive Differential Cross-Section For Heavy Quark Production In Hadronic Collisions," Nucl. Phys. B327, 49 (1989); Erratum B335 260 (1990).

[29] A. D. Martin, W. J. Stirling, and R. G. Roberts, "New information on parton distributions," Phys. Rev. D 47, 867 (1993).

[30] C. Peterson, D. Schlatter, I. Schmitt, and P. Zerwas, "Scaling Violations In Inclusive $e^{+} e^{-}$Annihilation Spectra," Phys. Rev. D 27, 105 (1983).

[31] CDF Collaboration, F. Abe et al., "The Cdf Detector: An Overview," Nucl. Instrum. Methods Phys. Res., Sect. A 271, 387 (1988).

[32] S. Tkaczyk et al., "The CDF Silicon Vertex Detector" Nucl. Instr. Meth. A342, 240250 (1994); D. Amidei et al., "The Silicon Vertex Detector of the Collider Detector at Fermilab" Nucl. Instr. Meth. A350, 73-130 (1994); P. Azzi et al., "SVX', the New CDF Silicon Vertex Ditector" Nucl. Instrum. Methods Phys. Res., Sect. A 360, 137 (1995).

[33] F. Snider et al., "The CDF Vertex Time Projection Chamber System," Nucl. Instrum. Meth. A268, 75 (1988). This is a reference for the previous generation of the device. The replacement for the Run I (1992-1996) data-taking period has more modules, each with a shorter drift length, but otherwise similar.

[34] F. Bedeschi et al., "Design and Construction of the CDF Central Tracking Chamber" Nucl. Instrum. Methods Phys. Res., Sect. A 268, 50 (1988).

[35] L. Balka et al., "The CDF Central Electromagnetic Calorimeter" Nucl. Instrum. Methods Phys. Res., Sect. A 267, 272 (1988). 
[36] S. Bertolucci et al., "The Cdf Central And Endwall Hadron Calorimeter," Nucl. Instrum. Meth. A267, 301 (1988).

[37] D. Amidei et al., "A two level FASTBUS based trigger system for CDF" Nucl. Instrum. Methods Phys. Res., Sect. A 269, 51 (1988); J. T. Carol et al., ibid. 300, 552 (1991).

[38] G. W. Foster et al., "A fast hardware track-finder for the CDF central tracking chamber" Nucl. Instrum. Methods Phys. Res., Sect. A 269, 82 (1988).

[39] P. Avery, K. Read, and G. Trahern, Cornell Internal Note CSN-212, 1985 (unpublished). We used version 6.1.

[40] T. Affolder et al. [CDF Collaboration], "Measurement of $b$ quark fragmentation fractions in p anti-p collisions at sqrts $=1.8 \mathrm{TeV}$," Phys. Rev. Lett. 84, 1663 (2000) [hep-ex/9909011].

[41] CDF Collaboration, F. Abe et al., "Observation of $B^{+} \rightarrow \psi(2 S) K^{+}$and $B^{0} \rightarrow$ $\psi(2 S) K^{*}(892)^{0}$ decays and measurements of B meson branching fractions into $J / \psi$ and $\psi(2 S)$ final states", Phys. Rev. D 58, 072001 (1998) hep-ex/9803013.

[42] J. Chrin, Z. Phys. C 36, 163 (1987). This value encompasses more recent measurements including OPAL Collaboration, G. Alexander et al., Phys. Lett. B 364, 93 (1995) and ALEPH Collaboration, D. Buskulic et al., ibid. 357, 699 (1995). 


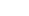

